\title{
Erweiterte Analyse der thermischen Zustände in Straßenbefestigungen unter besonderer Berücksichtigung klimatischer Einflüsse und stoffspezifischer Materialparameter
}

Extended analysis of the thermal conditions in road pavements with particular consideration of climatic conditions and material-specific properties

Markus Clauß

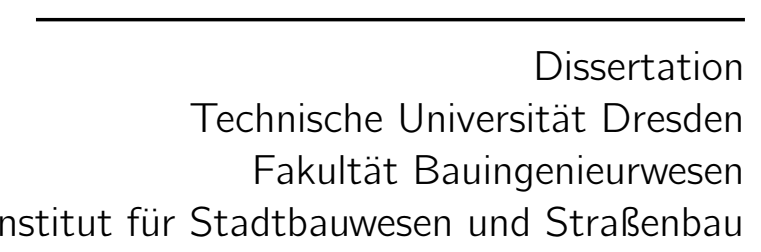

Tag der Einreichung: 08.10.2020

Tag der Verteidigung: 16.03.2021

Gutachter:

Prof. habil. Dr.-Ing. Frohmut Wellner Technische Universität Dresden Institut für Stadtbauwesen und Straßenbau

Prof. Dr.-Ing. Wolfram Ressel Universität Stuttgart Institut für Straßen- und Verkehrswesen Lehrstuhl für Straßenplanung und Straßenbau

Prof. Dr.-Ing. Martin Radenberg Ruhr-Universität Bochum Institut für Infrastruktur und Umwelt Lehrstuhl für Verkehrswegebau 


\section{Kurzfassung}

Das Nachweisverfahren für die Berechnung der Dauerhaftigkeit von Verkehrsflächen in Asphaltbauweise beruht auf der Bewertung der mechanischen Beanspruchungen, hervorgerufen durch die Verkehrslasten und der Temperatur. Die Materialkennwerte der Asphalte sind stark von der Temperatur abhängig und somit ist eine möglichst exakte Beschreibung der thermischen Zustände von Straßenbefestigungen während der Nutzungszeit von hoher Bedeutung. Die in Deutschland gültigen Prognose- und Dimensionierungsverfahren berücksichtigen standardisierte Temperaturprofile, welche die Temperaturzustände in der Straßenbefestigung in Abhängigkeit der Oberflächentemperaturen charakterisieren. Randparameter wie die Stoffeigenschaften der Baustoffe oder die Geometrie der Straßenkonstruktionen haben danach keinen Einfluss auf die Wärmetransportvorgänge und die daraus ermittelten charakteristischen Temperaturzustände.

In den vergangenen Jahren war der Einfluss des Klimawandels auf die lokalen klimatischen Bedingungen messbar. Besonders Dürren und Hitzewellen im Sommer führen zu deutlichen Schäden an der Natur. Dies lässt vermuten, dass die Änderung in Folge des Klimawandels einen erheblichen Einfluss auf die Temperaturzustände von Straßenbefestigungen haben.

Der Schwerpunkt der vorliegenden Arbeit lag auf der Bestimmung eines Verfahrens, mit dessen Hilfe die klimatischen Einflüsse, die Geometrie der Straßenkonstruktion und die stoffspezifischen Materialparameter berücksichtigt werden können. Dazu wurden Simulationsergebnisse der thermischen Zustände von Straßenkonstruktion ausgewertet und ein erweitertes Analyseverfahren zur Bestimmung von repräsentativen Temperaturzuständen entwickelt. Mithilfe eines Clusterverfahrens konnten anhand der stündlichen Temperaturzustände maßgebende Profile (Mittelwertfunktionen) abgeleitet werden, welche die Charakteristiken der Straßenkonstruktion berücksichtigen. Die Mittelwertfunktionen weisen gegenüber den bisherigen Ansätzen eine deutlich verminderte Abweichung zwischen den realen Temperaturzuständen und den zugehörigen synthetisch generierten Temperaturverläufen auf.

Mit dem Verfahren der Mittelwertfunktionen wurden detaillierte Analysen der Vulnerabilität der Verkehrsinfrastruktur in Deutschland auf Basis der prognostizierten Einflüsse infolge des Klimawandels durchgeführt. Zu diesem Zweck wurde für jeden Landkreis in Deutschland eine Simulation der thermischen und mechanischen Beanspruchungen durchgeführt und für die Ergebnisse der Nachweis auf Ermüdungsrissbildung an der Unterseite der Asphalttragschicht sowie der Entwicklung der Spurrinnentiefe in Anlehnung an das Dimensionierungsverfahren nach den RDO Asphalt (2009) geführt.

Abschließend wurde an mehreren Fallbeispielen die Anwendbarkeit des Verfahrens der Mittelwertfunktionen gezeigt.

Als Schlussfolgerung dieser Arbeit kann abgeleitet werden, dass die thermischen Zustände in Straßenbefestigungen durch Verwendung der Mittelwertfunktionen mit deutlich geringeren Abweichungen und somit einer höheren Güte im Dimensionierungsverfahren verwendet werden können. Weiterhin wurde festgestellt, dass der Klimawandel einen essenziellen Einfluss auf die Dauerhaftigkeit der Straßenbefestigungen in Asphaltbauweise in ganz Deutschland haben. Es ist zwingend notwendig, Maßnahmen aus den Erkenntnissen dieser Arbeit abzuleiten, um die Resilienz der Infrastruktur gegenüber dem Klimawandel zu erhöhen. 


\section{Abstract}

The procedure for dimensioning of asphalt pavements is based on the evaluation of the mechanical stresses caused by traffic loads and temperature effects. The material characteristics of asphalt are strongly dependent on temperature and hence, it is of utmost importance that we acquire the most accurate description of the thermal conditions of asphalt pavements during the life span. The prediction and dimensioning methods take standardised temperature profiles into account, which determine the temperature conditions in the asphalt layers depending on the characteristic surface temperature. Boundary parameters such as the material properties of building materials or the geometry of the pavements, therefore, have no influence on the heat transfer processes and the characteristic temperature conditions derived from them.

In recent years, the influence of climate change on local climatic conditions has been quantifiable. Especially, droughts and heat waves in summer have caused significant damage to nature. This is indicative that the changes resulting due to climate change will indeed have a significant impact on the temperature conditions of asphalt pavements in the future.

The main focus of this dissertation was the determination of a method that takes into account the climatic influences, the geometry of the road construction and the specific properties of the materials. For this purpose, the thermal conditions of pavements were evaluated by simulating results and an extended analysis method for the determination of relevant temperature conditions was developed. With the help of a cluster method, significant profiles (i.e. mean temperature functions) could be derived from the hourly temperature states, which take the characteristics of the road construction into account. Compared to the previous approaches, the mean temperature functions show a significantly reduced deviation between the real temperature states and the corresponding simulation-generated temperature curves.

Using the method of mean temperature functions, detailed analysis of the vulnerability of the transport infrastructure in Germany were carried out on the basis of the predicted future impacts of climate change. For this purpose, a simulation of the thermal and mechanical stresses was carried out for each district in Germany. The results acquired were used to prove the existence of fatigue cracking on the underside of the asphalt base layer and the development of the rut depth based on the dimensioning method according to the RDO Asphalt (2009).

As a conclusion of this dissertation, it can be deduced that the thermal conditions in road pavements can be utilised in the dimensioning process by using the mean temperature functions with significantly lower deviations, which would result in superior quality pavements. Furthermore, it was found that climate change has an essential influence on the durability of asphalt pavements throughout Germany. It is imperative to adopt measures from the findings of this work in order to increase the resilience of the German infrastructure to climate change. 


\section{Inhaltsverzeichnis}

1 Motivation und Zielstellung 1

2 Klimatische Bedingungen $\quad 5$

2.1 Ausgangssituation . . . . . . . . . . . . . . . . . . 5

2.2 Klimawandel . . . . . . . . . . . . . . . . . . . . . . . . 7

2.3 Emissionsszenarien . . . . . . . . . . . . . . . . . . . . . . 8

2.4 Prognose und Bewertung . . . . . . . . . . . . . . . . . . . . . . . 10

2.5 Einfluss auf Straßenkonstruktionen . . . . . . . . . . . . . . . . . . 13

3 Dimensionierung von Straßenbefestigungen $\quad \mathbf{1 5}$

3.1 Verfahrensbeschreibung . . . . . . . . . . . . . . . . . . . 15

3.2 Nachweis auf Ermüdungsrissbildung . . . . . . . . . . . . . . . 17

3.3 Nachweis auf Spurrinnenentwicklung . . . . . . . . . . . . . . . . . . 18

3.4 Hauptdehnungen . . . . . . . . . . . . . . . . . . . . . . . . . 20

3.5 Tagesganglinie der Verkehrsstärken . . . . . . . . . . . . . . . . . . . 24

4 Simulation der thermischen Beanspruchungen $\quad 31$

4.1 Allgemeines . . . . . . . . . . . . . . . . . . . . . . . . 31

4.2 Modellbeschreibung . . . . . . . . . . . . . . . . . . . . . . . . . . 31

4.3 Eingangsparameter . . . . . . . . . . . . . . . . . . . . 35

4.3.1 Klimadaten . . . . . . . . . . . . . . . . . . . . . . . . . . 35

4.3.2 Thermophysikalische Materialparameter und Geometrie . . . . . . . 37

4.4 Simulationsergebnisse . . . . . . . . . . . . . . . . . . . . . . . . . 39

4.4.1 Maximaltemperaturen ... . . . . . . . . . . . . . . . . 39

4.4.2 Anzahl an heißen Tagen in der Asphaltbefestigung . . . . . . . . . 41

4.4 .3 Mittlere Temperaturen . . . . . . . . . . . . . . . . . . . . . 43

4.4.4 Anzahl an Tage mit Frost in der Straßenbefestigung . . . . . . . . 46

4.4 .5 Zusammenfassung . . . . . . . . . . . . . . . . . . . 49

5 Clusteranalyse $\mathbf{5 3}$

5.1 k-Means Algorithmus . . . . . . . . . . . . . . . . . . . . . . . 54

5.2 Vergleich der Verfahren . . . . . . . . . . . . . . . . . . . . . . 58

6 Klimafolgen für Asphaltbefestigungen $\quad \mathbf{6 5}$

6.1 Motivation . . . . . . . . . . . . . . . . . . . 65

6.2 Risiko Ermüdungsrissbildung . . . . . . . . . . . . . . . . . 66 
6.3 Risiko Spurrinnenentwicklung . . . . . . . . . . . . . . . . . . . . . . . . . . 71

6.4 Zusammenfassung . . . . . . . . . . . . . . . . . . . 75

$\begin{array}{lll}7 & \text { Fallbeispiele } & \mathbf{7 7}\end{array}$

7.1 Temperierung von Straßenbefestigungen . . . . . . . . . . . . . 77

7.2 Hitzeperiode . . . . . . . . . . . . . . . . . . . . . . . . . 86

7.3 Phase Change Materials . . . . . . . . . . . . . . . . . . . . . . . . 91

8 Zusammenfassung $\quad 99$

$\begin{array}{lr}\text { Anhang } & 119\end{array}$

A Klimarisiken . . . . . . . . . . . . . . . . . . . . . . . . . . . . 121

A.1 Change Point Analyse . . . . . . . . . . . . . . . . . . 121

A.2 Klimaparameter . . . . . . . . . . . . . . . . . . . . . 122

B Clusteranalyse . . . . . . . . . . . . . . . . . . . . . . 167

B.1 Mittelwertfunktionen . . . . . . . . . . . . . . . 167

B.2 Normierte charakteristische Temperaturprofile . . . . . . . . . . 174

B.3 Temperaturprofile nach den RDO Asphalt . . . . . . . . . . . . 181

B.4 Dimensionierung . . . . . . . . . . . . . . . . . . . . . . . . . . 185

C Dimensionierungsberechnungen . . . . . . . . . . . . . . . . 187

C.1 Miner-Summe . . . . . . . . . . . . . . . . . . . . . . . . . . 187

C.2 Spurrinnentiefe . . . . . . . . . . . . . . . . . . 200

D Anpassungsstrategie . . . . . . . . . . . . . . . . . . . . . . . . 213

D.1 Fallbeispiel PCM . . . . . . . . . . . . . . . . . . . . . . . . . . 214 


\section{Motivation und Zielstellung}

Die mechanische Stabilität von Straßenbefestigungen aus Asphalt und deren zu erwartende Nutzungszeiträume sind über die Temperaturabhängigkeit der Materialeigenschaften sehr stark von witterungsbedingten Umgebungseinflüssen abhängig.

Aus den Wetteraufzeichnungen der letzten Jahrzehnten konnte man einen deutlichen Anstieg der durchschnittlichen Jahresmitteltemperaturen erkennen. In Abbildung 1.1 ist die Anomalie der mittleren jährlichen Oberflächentemperatur über dem Land und den Ozeanen zur Referenztemperatur der Erdoberfläche (1980 bis 2015) dargestellt. Die Beobachtungen des Erdklimas des vergangen Jahrhunderts haben gezeigt, dass ein kontinuierlicher Anstieg der Oberflächentemperatur innerhalb des Betrachtungszeitraums festgestellt werden konnte.

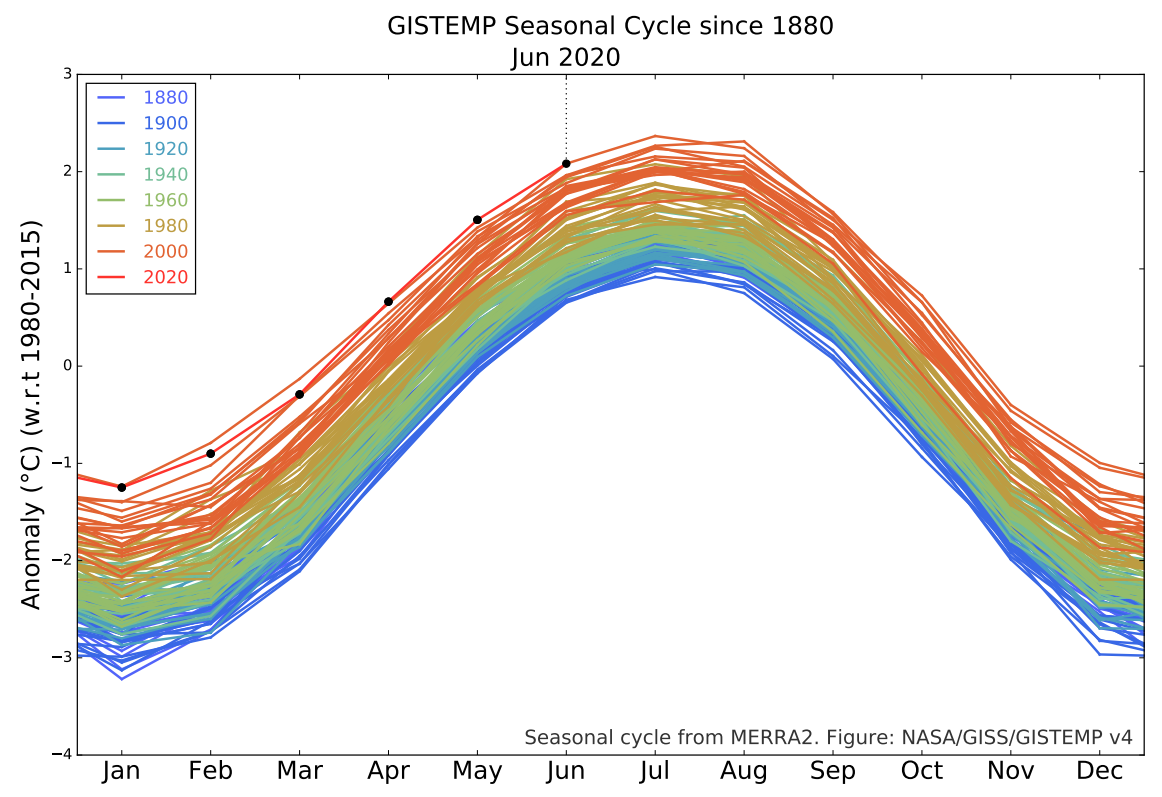

Abbildung 1.1

Temperaturanomalie der Oberflächentemperatur der Erde bezogen auf die Periode 1980 bis 2015, NASA (2020)

Wetterextreme nehmen nachweislich in Dauer und Intensität zu. Die meteorologischen Beobachtungen der Oberflächentemperaturen der Erde durch Wetterdienste ergab genau dieses Phänomen. Seit 2015 konnte für jedes Jahr ein neuer Rekordwert der mittleren Oberflächentemperatur aufgezeichnet werden. Die fünf höchsten Temperaturanomalien 


\section{Motivation und Zielstellung}

wurden in den Jahren $2016\left(0,99^{\circ} \mathrm{C}\right), 2019\left(0,95^{\circ} \mathrm{C}\right), 2015\left(0,93^{\circ} \mathrm{C}\right), 2017\left(0,91^{\circ} \mathrm{C}\right)$ und $2018\left(0,83^{\circ} \mathrm{C}\right)$ von der National Oceanic and Atmospheric Administration (NOAA) gemessen und von den National Centers for Environmental Information (NCEI) veröffentlicht. Die Messungen zeigen, dass sich Europa besonders stark erwärmt hat.

Die Ergebnisse von regionalen Klimasimulationen für Deutschland ergaben, dass es im Allgemeinen zu einer Erhöhung der Temperaturen bei einer gleichzeitigen Zunahme der Klimavariabilität kommt (Fischer und Schär, 2010). Die Kombination dieser beiden Effekte führt dazu, dass die Häufigkeit kalter Witterungsextreme sich kaum verändert, aber die Intensität und die Häufigkeit von warmen Extremen zunehmen wird (Hartmann u. a., 2013).

Trotz zahlreicher Klimaabkommen, welche eine Mehrzahl an Ländern weltweit ratifiziert haben, nimmt der $\mathrm{CO}_{2}$-Gehalt in der Atmosphäre stetig zu. Im Jahr 2017 konnten Spitzenwerte in der Konzentration gemessen werden. Werte, welche seit mindestens 800.000 Jahren nicht nachgewiesen werden konnten. Bedingt durch die klimatischen Veränderungen schmelzen die Eismassen besonders schnell. Der Meeresspiegelanstieg bedroht zahlreiche Städte, Küsten, Regionen und Inseln. Auch für Deutschland ergeben sich Konsequenzen. Die Ozeane haben sich weiterhin deutlich erwärmt, da ein Großteil der globalen Erwärmung in den Ozeanen gespeichert wird (IPCC, 2014).

Der Klimawandel erfordert ein globales Umdenken in vielen Bereichen. Wichtig dabei ist, Schlussfolgerungen basierend auf wissenschaftlichen Analysen zu den Schadenspotenzialen zu erstellen und konsequent weiterzuentwickeln. Exemplarisch für diese Risikobewertungen konnte im Bereich der Medizin eine erhöhte Sterblichkeitsrate bei Hitzewellen nachgewiesen werden (Robine u. a., 2008; Lozan u. a., 2014). In einigen wissenschaftlichen Arbeiten wurde ermittelt, dass auch Folgen für den Verkehrsbereich bestehen (Gudipudi, Underwood und Zalghout, 2017; Clauß, Kayser und Wellner, 2017; Wistuba und Walther, 2013; Qiao u. a., 2015; Viola und Celauro, 2015). Im Rahmen der Untersuchungen wurden meist lokale Prognosen zur Abschätzung der Klimafolgen durchgeführt. Die Studien deuten an, dass Schädigungen an Straßenbefestigungen in Asphalt- und Betonbauweise auftreten können.

Doch bereits in der aktuellen Zeit können Indizien für die Entwicklung gefunden werden (Abbildung 1.2). In den Medien konnte man weltweit Berichte über Folgen von hohen Temperaturen für die Verkehrsinfrastruktur lesen. Ein besonders extremes Ereignis wurde während der Hitzewelle 2015 in Indien dokumentiert. Bedingt durch die andauernden hohen Temperaturen in Neu-Delhi haben sich in bestimmten Bereichen extrem große plastische Verformungen infolge der Verkehrsbelastung an der Oberseite der Fahrbahn eingestellt. In der Fachpresse konnte für Deutschland eine erhöhte Wahrscheinlichkeit des Auftretens von Schlaglöchern - im Artikel wurde von Hitzeblasen an der Oberseite der Fahrbahn gesprochen festgestellt werden (Neubauer, 2015). Doch auch die Infrastruktur der Bahn oder Betonbefestigungen waren betroffen. Die genannten Beispiele stellen momentan noch ein sehr seltenes Schadensbild dar. In Anbetracht der projizierten Anomalie der Oberflächentemperatur der Erde muss davon ausgegangen werden, dass Schädigungen weltweit häufiger und stärker auftreten. 


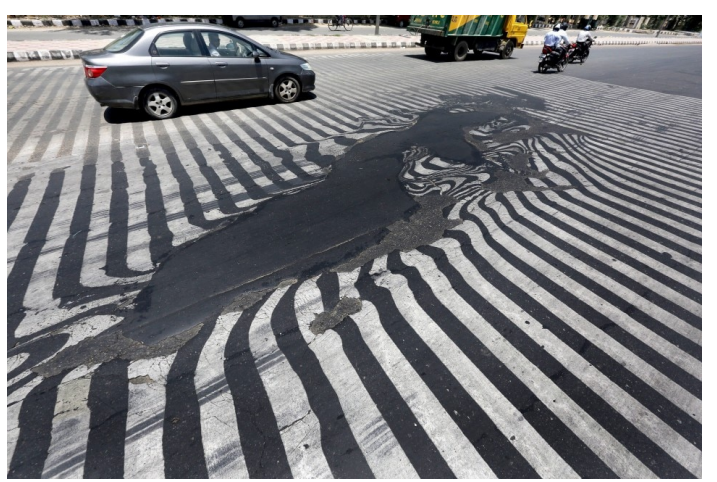

(a) Schädigungen durch Hitze an der Fahrbahnoberseite in Indien

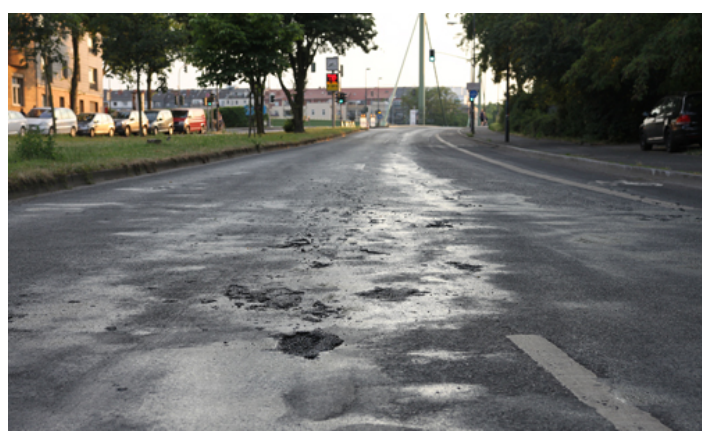

(c) Schlaglöcher in der Asphaltdeckschicht

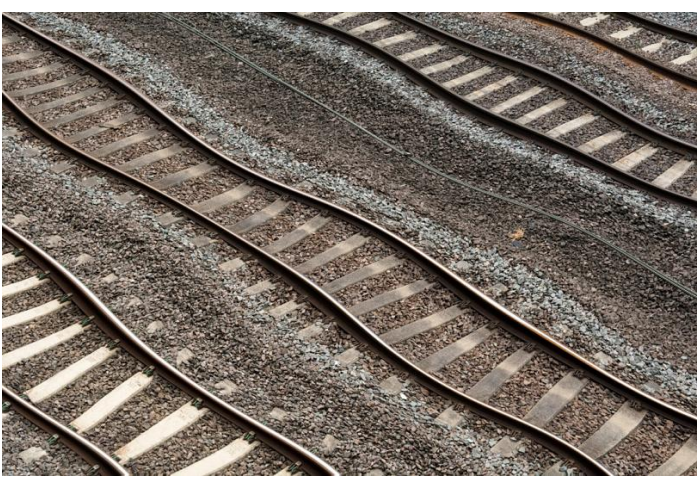

(b) Verformung des Gleisbettes infolge einer Hitzewelle in Schweden

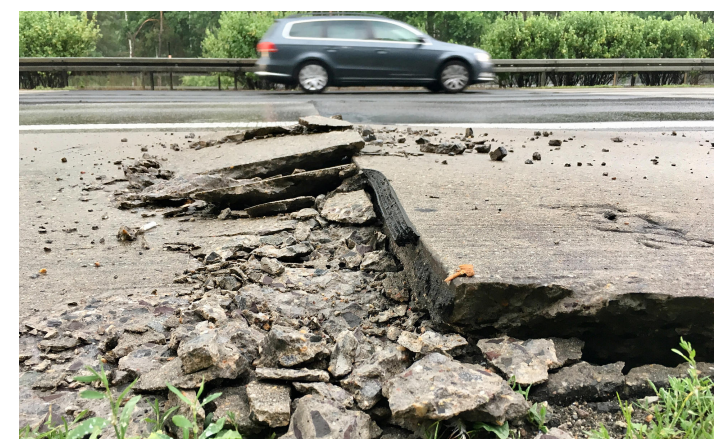

(d) Aufbruch der Fahrbahnplatte

Abbildung 1.2

Beispiele für Schädigungen durch Hitze an der Verkehrsinfrastruktur; (a) Harvey (2017) (b) Euronews (2019), (c): Neubauer (2015), (d): Wetschera (2019)

Die Erfahrungen aus den letzten Jahren zeigen, dass die Klimafolgen einen erheblichen Einfluss auf die Dauerhaftigkeit von Straßenkonstruktionen aufweisen. Die Weiterentwicklung von praxisnahen Berechnungsverfahren für die Dimensionierung und Prognose von Straßenbefestigungen in Asphaltbauweise muss kontinuierlich fortgeführt werden. Dabei ist besonders wichtig, dass relevante Einflussgrößen detektiert und in das Verfahren integriert werden.

In der vorliegenden Arbeit wurden daher detaillierte Untersuchungen der klimatischen Einflüsse auf die Dauerhaftigkeit von Straßenkonstruktionen durchgeführt. Diese erfolgten mit einer Modellierung des thermischen Verhaltens von Straßenkonstruktionen in Asphaltbauweise, basierend auf Klimaprojektionen für den Raum Deutschland. Die Berechnungen der thermischen Beanspruchungen wurden statistisch aufbereitet und anhand von repräsentativen Temperaturzuständen die mechanische Beanspruchungen in der Straßenkonstruktion simuliert. Auf Basis der ermittelten Dehnungen in der Straßenbefestigung wurden Prognoserechnungen über die Entwicklung von Spurrinnen sowie Ermüdungsrissen in Abhängigkeit des Zeitpunk- 
tes der Verkehrsfreigabe geführt. Dies ermöglichte die Bestimmung der Vulnerabilität der Verkehrsinfrastruktur in Asphaltbauweise für Deutschland.

Weiterhin wurde das Dimensionierungs- und Prognoseverfahren überprüft und ggf. Anpassungen abgeleitet. Im besonderen Fokus der vorliegenden Arbeit stand deshalb die statistische Bewertung der thermischen Zustände, da die thermophysikalischen Materialeigenschaften, die Geometrie der Straßenkonstruktionen sowie die klimatischen Bedingungen einen Einfluss auf die Temperaturzustände von Straßenkonstruktionen aufweisen. Abschließend wurde anhand von Fallbeispielen die Anwendbarkeit des Verfahrens dargestellt. 


\section{Klimatische Bedingungen}

\subsection{Ausgangssituation}

Der Begriff Wetter bezeichnet den physikalischen Zustand der Atmosphäre zu einem spezifischen Zeitpunkt an einem bestimmten Ort. Der Zeitraum umfasst dabei wenige Stunden bis hin zu einigen Tagen. Die Parameter wie die Lufttemperatur, die Windgeschwindigkeit oder die Regenmenge werden durch meteorologische Beobachtungen regelmäßig gemessen und aufgezeichnet.

Klima ist hingegen der mittlere Zustand der Atmosphäre über einen längeren Zeitraum an einem spezifischen Ort. Die World Meteorological Organization (WMO) spricht dabei von einem gemittelten Wetter, welches über die Mittelwerte und die Varianzen von relevanten Beobachtungen von Parametern bestimmt werden. Der zeitliche Horizont der Beobachtungen ist zwischen mehreren Monaten, über Jahre bis hin zu Jahrtausenden oder Millionen von Jahren. Ein üblicher Betrachtungszeitraum ist mit 30 Jahren angegeben. Statistische Zustände, wie Mittelwert und Extremwerte, der Atmosphäre charakterisieren das Klima.

Die Begriffe Wetter und Klima sind stark miteinander verbunden. Wetter beschreibt die Beobachtungen der Atmosphäre in einem kurzen Zeitraum, wohingegen Klima langräumige Beobachtungen statistisch analysiert. Aus wenigen extremen Sommern kann nicht abgeleitet werden, dass sich das Klima hin zu wärmeren Temperaturen verändert hat. Aus Messungen der Lufttemperatur, welche über mehrere Jahrzehnte kontinuerlich erfolgt sind, kann jedoch ein Trend und somit eine Aussage über die Änderung des Klimas abgeleitet werden. Gleichermaßen kann man aus den Daten von Klimasimulationen nicht auf die Wetterlage an einem spezifischen Tag und einem bestimmten Ort schließen.

Die meteorologischen Beobachtungen unterscheiden sich in lokalen Bereichen deutlich von den gemittelten Annahmen über die gesamte Erdoberfläche. In Deutschland gibt es, seit der Gründung eines staatlichen Wetterdienstes im Jahr 1848, kontinuierliche und einheitliche Aufzeichnungen des Wetters. Die Anzahl der Messstationen wurde seit der Gründung sukzessive vergrößert, neue Messgeräte zur Erfassung weiterer Parameter installiert und die Aufzeichnungsrate erhöht. Weiterhin gibt es Beobachtungen des globalen und regionalen Wetters aus dem Weltall. Somit stehen Daten zur Verfügung, auf deren Basis statistische Auskünfte für kleinräumige Strukturen möglich sind.

Aus den Zeitreihen der Wetterbeobachtungen können Trends für Deutschland oder spezifische Regionen abgeleitet werden. In Abbildung 2.1 ist die Änderung der Temperatur bezogen auf 


\section{Klimatische Bedingungen}

den Zeitraum 1961 bis 1990 für Deutschland dargestellt. Im langjährigen linearen Trend ist die Temperatur von -0,80 K im Jahr 1981 auf 0,90 K im Jahr 2019 gestiegen. Die Messdaten für 2019 zeigen eine Anomalie von der mittleren Jahrestemperatur von mehr als 2,00 K. Deutschland erwärmte sich in diesem Zeitraum etwas stärker als der weltweite Durchschnitt. Rekordverdächtig war auch das Überschreiten der $40,00^{\circ} \mathrm{C}$-Schwelle der Lufttemperatur an drei aufeinanderfolgenden Tagen (24. bis 26. Juli 2019) in einigen Bereichen im Westen Deutschlands (Kaspar und Friedrich, 2020). Weiterhin wurde festgestellt, dass 11 der 12 Monate wärmer waren als die Mittelwerte der Referenzperiode. Nur der Mai war mit $1 \mathrm{~K}$ bis $2 \mathrm{~K}$ kühler. Im Juni wurde ein um $5 \mathrm{~K}$ höherer Mittelwert gemessen. Eine starke Trockenheit konnte seit 2018 in großen Teilen von Deutschland beobachtet werden.

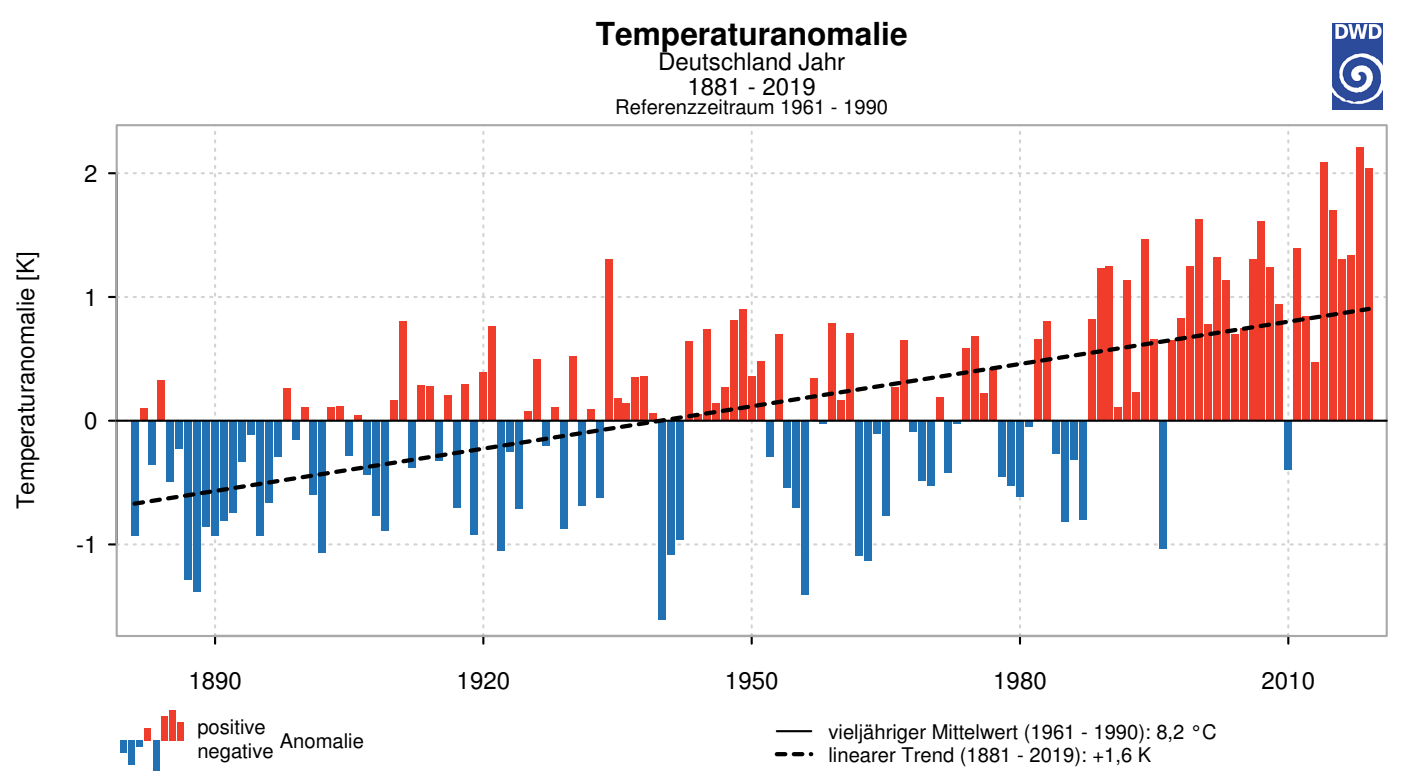

Abbildung 2.1

Anomalie der Lufttemperatur für den Zeitraum von 1881 bis 2019 (Deutscher Wetterdienst)

Die Beobachtungen des Wetters deuten an, dass Extremwetterereignisse häufiger vorkommen und die Intensität zunimmt. Eine statistische Gewissheit ist, bedingt durch die Seltenheit der extremen Ereignisse, nur schwer möglich (Jacob, Ries und Weber, 2016). Das Auftreten von extremen Wetterbedingungen beeinflusst jedoch häufig die Gesundheit von Mensch oder führt zu Schädigungen an der Natur oder Infrastruktur. Aus den Temperaturmessungen können zum Beispiel die Anzahl an Tagen mit einer Tageshöchsttemperatur größer gleich $25^{\circ} \mathrm{C}$ bestimmt werden. In Abbildung 2.2 sind die gemessenen Daten grafisch dargestellt. Bis ungefähr in die 1990er Jahre war in einer Vielzahl an Jahren die Anzahl des Vorkommens von Sommertagen - also Tage mit einer maximalen Lufttemperatur größer gleich $25^{\circ} \mathrm{C}-$ kleiner (negativ) als die durchschnittliche Anzahl an Sommertagen im Zeitraum 1961 bis 1990. Analog zu der beschriebenen Erhöhung der mittleren Temperaturen in Deutschland 
stieg auch die Anzahl der jährlichen Sommertage kontinuierlich an. Die Auswertung zeigt, dass im Jahr 2018 ein Rekord von nahezu 50 zusätzlichen Sommertagen zum langjährigen Durchschnittswert von 27,3 Tagen gemessenen wurde. Der lineare Trend der Messreihen ergibt, dass sich die mittlere Anzahl an Tagen um ca. 22,5 Tagen seit 1951 erhöht hat. Die Dauer von sommerlichen Hitzewellen hat sich seit den 1880er Jahren nahezu verdreifacht (Brasseur, Jacob und Schuck-Zöller, 2017, S. 54f).

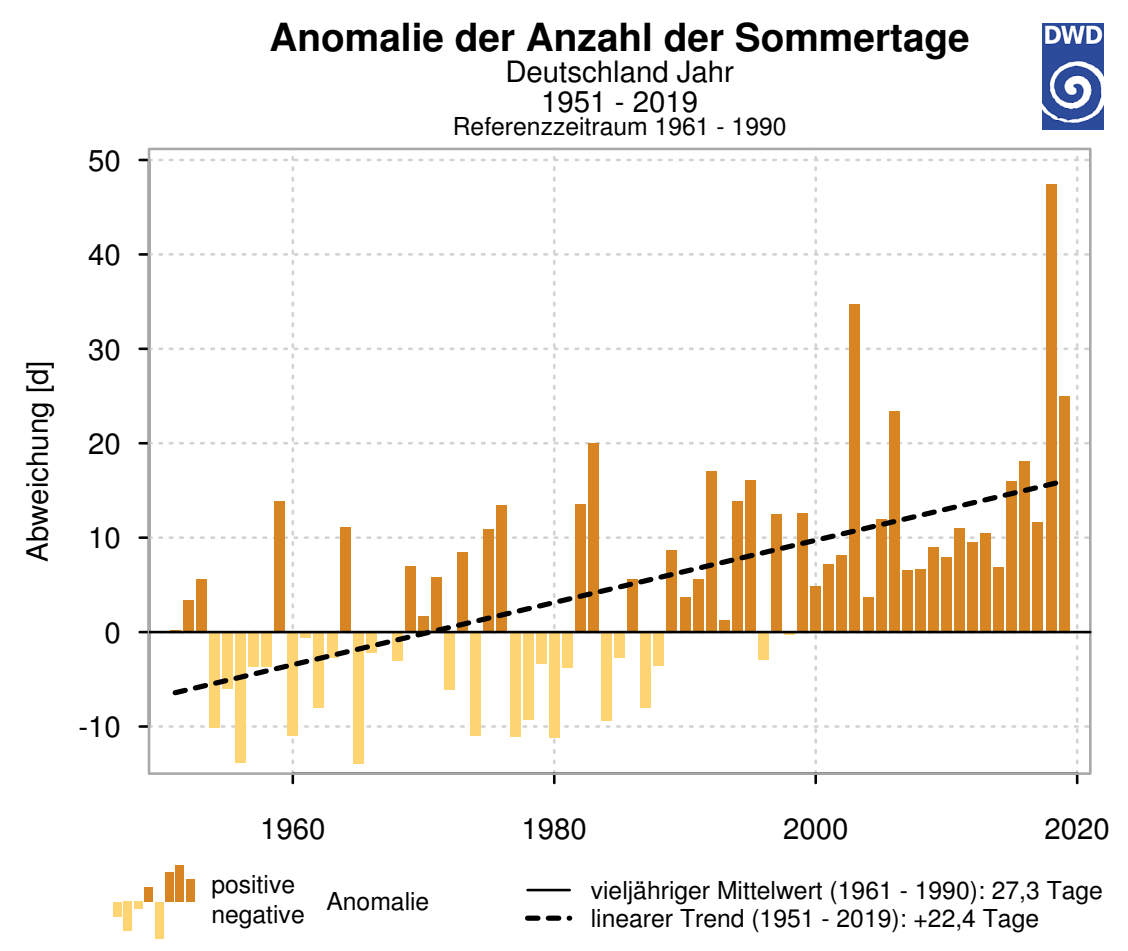

Abbildung 2.2

Anomalie der Anzahl der Sommertage für Deutschland (Deutscher Wetterdienst)

\subsection{Klimawandel}

Die Beobachtungen des Wetters zeigen, dass sich weltweit das Klima in der Vergangenheit geändert hat. Es erscheint notwendig, diese Veränderungen abschätzen zu können, um die ökonomischen und gesellschaftlichen Folgen zu bestimmen und ggf. Anpassungsstrategien und Maßnahmen gegen das Fortschreiten abzuleiten. Um fundierte Aussagen treffen zu können, ist es nicht ausreichend die Beobachtungen des vergangenen Wetters statistisch zu bewerten. Viel entscheidender ist es, die zukünftigen Änderungen möglichst gut vorhersagen zu können. Ein Hauptinstrument in der Klimaforschung bilden für diesen Zweck numerische 


\section{Klimatische Bedingungen}

Modelle. Die Klimatologie ist eine interdisziplinäre Wissenschaft mit einer starken Verknüpfung zur Mathematik, Physik, Informatik, Ozeanografie, Chemie und Umweltwissenschaft. Es gibt auch Verbindungen zu den Wirtschaftswissenschaften und vielen weiteren Teildisziplinen. Diese Arbeitsweise ist notwendig, um eine Abstraktion der extrem komplexen Mechanismen des Wetters zu erstellen. Die Zusammenhänge der unterschiedlichsten Eingangsparameter können formal mit mathematischen Methoden modelliert werden. Die Lösung dieser Aufgaben kann nur mithilfe von Computersystemen erfolgen.

Die Entwicklung unterschiedlicher Klimamodelle hilft bei der Bewertung der klimatischen Veränderungen und erlaubt - auf Basis unterschiedlicher Eingangsparameter und Annahmen während des Berechnungszeitraums - politische Entscheidungen und deren Folgen mit wissenschaftlichen Methoden zu bewerten. Fragestellungen wie beispielweise nach dem Einfluss einer Erhöhung der Treibhausgaskonzentration in der Atmosphäre auf die globale Lufttemperatur, können mit Klimaprojektionen beantwortet werden. Klimamodelle bilden somit das wichtigste Werkzeug für die Beurteilung von Klimafolgen.

Viele aktuelle Fragestellungen wurden bereits in der Vergangenheit aufgeworfen. Bereits 1896 beschrieb der schwedische Chemiker und Physiker Svante August Arrhenius den positiven Rückkopplungseffekt, welcher durch eine Erhöhung der $\mathrm{CO}_{2}$-Konzentration in der Atmosphäre verursacht wird (Arrhenius, 1896). Er hatte berechnet, dass eine Verdoppelung der Konzentration von $\mathrm{CO}_{2}$ in der Atmosphäre eine Erhöhung der mittleren Oberflächentemperatur der Erde um $6 \mathrm{~K}$ verursacht (Gleichgewichtsklimasensitivität).

Das erste Klimamodell, welches auf physikalischen Grundlagen beruht, wurde 1961 von Manaba und Möller entwickelt (Manaba und Möller, 1961). Dieses eindimensionale Strahlungskonvektionsmodell bildet die Grundlage aktueller Klimamodelle. Die Entwicklung ist jedoch nicht stehen geblieben. Aktuelle Modelle sind dreidimensionale atmosphärische Zirkulationsmodelle, welche in der Wettervorhersage verwendet werden. Der hohe Detailgrad dieser Modelle erfordert eine enorme Rechenleistung, welche kontinuierlich weiterentwickelt und ausgebaut wird. Dieses Vorgehen ermöglicht zugleich eine Implementierung weiterer meist sehr rechenintensiver - physikalischer und chemischer Prozesse. Durch Verwendung von modernster Rechnerinfrastruktur konnte die räumlichen Auflösung der Klimasimulationen kontinuierlich verfeinert werden, was auch die Berücksichtigung von kleinräumigen Klimaunterschieden in Global- und Regionalmodellen ermöglicht.

\subsection{Emissionsszenarien}

Für die Durchführung von numerischen Simulationen müssen Start- und Randbedingungen definiert werden. Diese Daten werden im Bezug auf Klimasimulationen als Emissionsszenarien (kurz Szenarien) bezeichnet. Für den Vierten Sachstandsbericht des Intergovernmental Panel of Climate Change (IPCC) wurden die SRES-Emissionsszenarien für die Durchführung der Klimasimulationen verwendet. In der deutschen Literatur werden oft Ergebnisse aus dem Modellauf A1B, B1 oder A2 dargestellt. 
In Abbildung 2.3 sind sowohl die Änderungen der globalen Treibhausgas-Emissionen ( global GHG emissions) als auch die mittlere Änderung der Oberflächentemperatur der Erde vergleichend dargestellt. Es ist ersichtlich, dass die Eingangs- und Randparameter einen erheblichen Einfluss auf die Klimaparameter haben. Die unterschiedlichen Szenarien bilden die Entwicklung der Gesellschaft und deren politische Entscheidungen im Bezug z. B. auf Ökonomie, Ökologie oder die Energiewirtschaft ab.
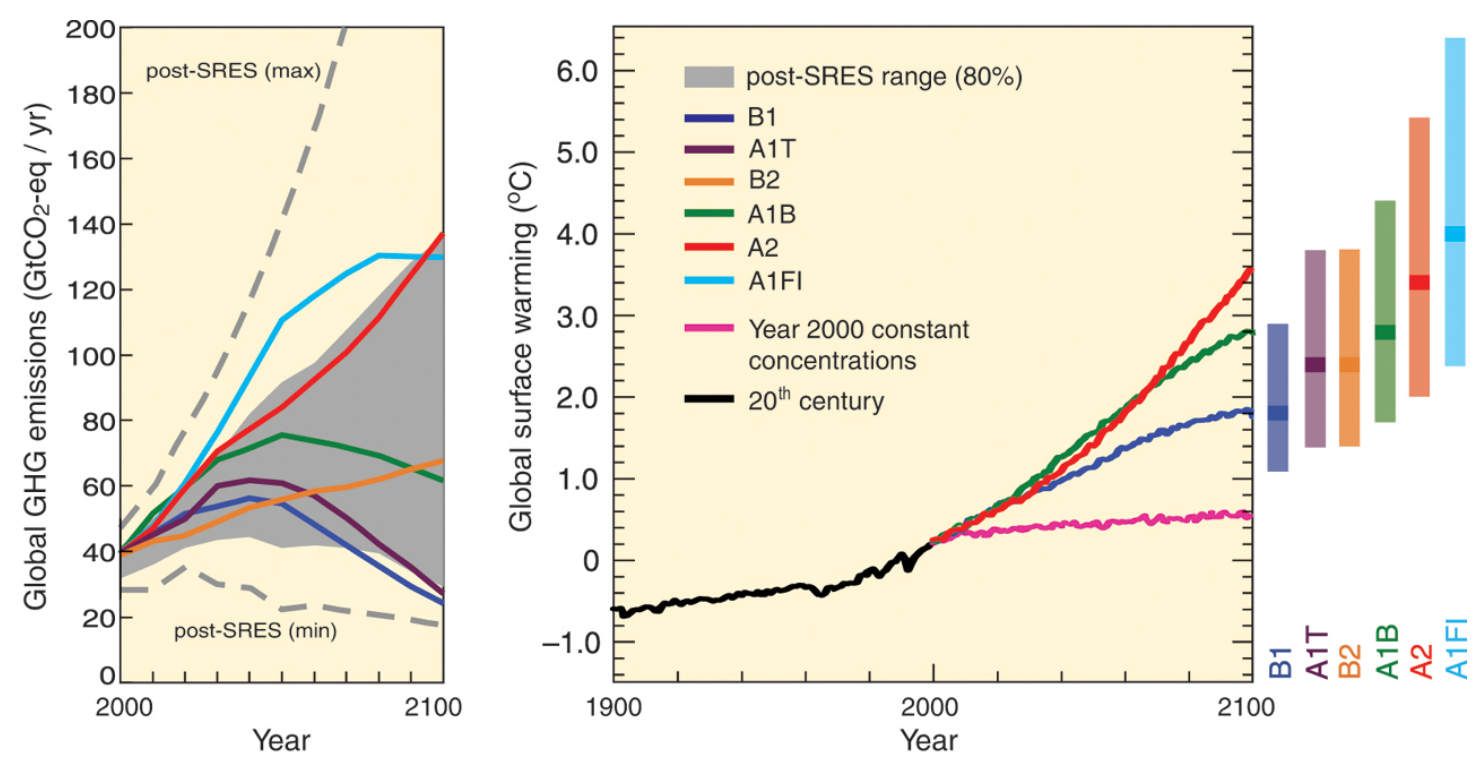

Abbildung 2.3

Darstellung der Treibhausgas-Emissionen nach den SRES-Emissionsszenarien und der resultierenden Temperaturanomalie, (IPCC, 2007, Abbildung SPM.5)

Im Bericht des IPCC wurden vier große Hauptszenarien definiert. In der vorliegenden Arbeit beruhen die Untersuchungen der Auswirkungen der klimatischen Effekte auf den Annahmen des Emissionsszenarios A1B. Dieses Szenario gehört zu der Szenario-Familie A1. Dabei wurde angenommen, dass die Entwicklung der zukünftigen Welt mit einem sehr starken Wirtschaftswachstum einhergeht. Die Weltbevölkerung wird bis Mitte des 21. Jahrhunderts zunehmen und sich dann rückläufig entwickeln. Die Nutzung von neuen Technologien wird verstärkt voranschreiten, wobei der Schwerpunkt auf der Entwicklung effizienter Systeme liegt. Dies bewirkt, dass sich Regionen wirtschaftlich annähern und somit eine Homogenisierung des Einkommens überregional erfolgt. Die Szenario-Familie A1 kann in drei Gruppen aufgeteilt werden. Für das Szenario A1B wird unterstellt, dass eine ausgewogene Nutzung von fossilen und nicht fossilen Energiequellen erfolgt. Die weiteren Szenario-Familien sind A2, B1 und B2. Auf eine gesonderte Betrachtung wird in dieser Arbeit verzichtet.

Für die Rechendurchläufe des fünften Sachstandsberichtes wurden die SRES-Emissionsszenarien überarbeitet und die Representative Concentration Pathways (RCPs) (Vuuren u. a., 2011; Wayne, 2013) anhand aktueller Forschungsergebnisse abgeleitet. Diese Emissionsszenarien beruhen auf der Entwicklung des Strahlungsantriebes der Atmosphäre. 


\section{Klimatische Bedingungen}

In Abbildung 2.4 sind die Strahlungsantriebe der SRES-Szenarien und der RCPs dargestellt. Das in der vorliegenden Arbeit untersuchte Szenario A1B liegt zwischen den RCP6.0 und $R C P 8.5$, entspricht also einer eher pessimistischen Annahme. Aus dem Diagramm ist ersichtlich, dass die Startwerte im Jahr 2020 der unterschiedlichen Annahmen etwas voneinander abweichen. Innerhalb der SRES-Szenarien wird von einem geringeren Strahlungsantrieb der Atmosphäre ausgegangen, was langfristig zu einem geringeren Anstieg der globalen Temperatur führt. Bei dem Szennario A1B hingegen, wird von starker Erhöhung der Treibhausgaskonzentration in den kommenden Jahrzehnten ausgegangen. Dies führt dazu, dass die simulierten Temperaturen in der nahen Zukunft stärker als bei weiteren SRES-Szenarien ansteigen wird.

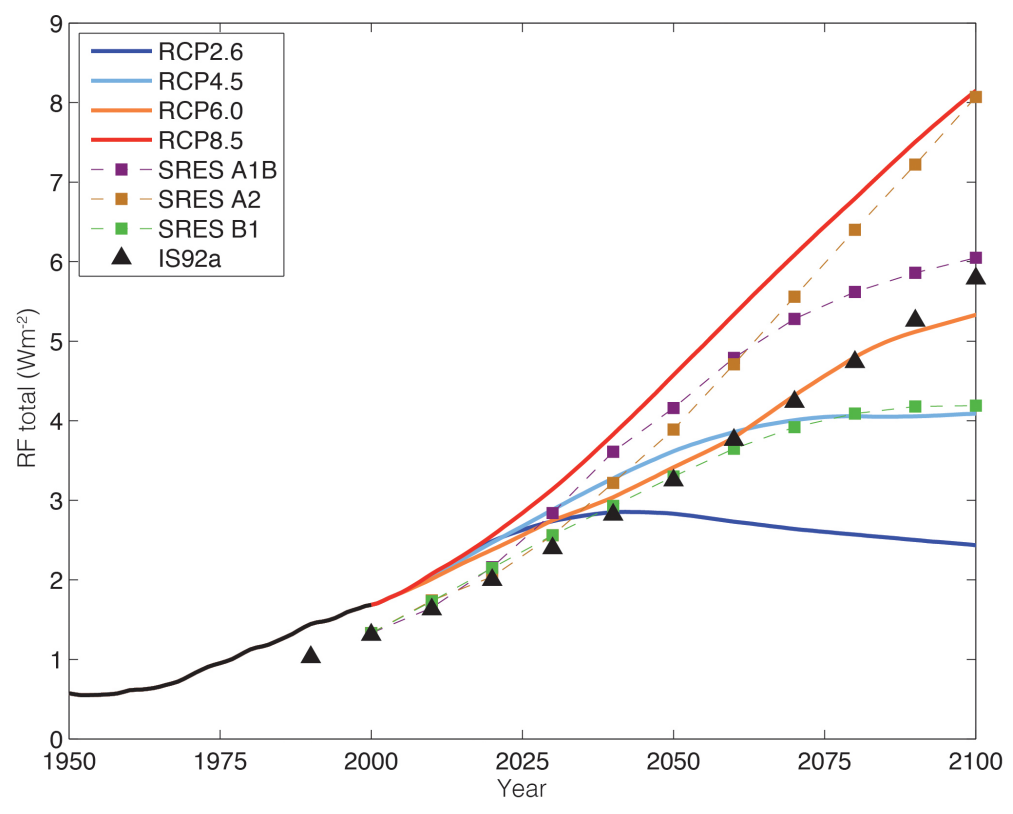

Abbildung 2.4

Veränderung des Strahlungsantriebes der Atmosphäre im Vergleich zwischen den SRESEmissionsszenarien zu den RCPs (IPCC, 2013, Abbildung 1.15, S. 146)

\subsection{Prognose und Bewertung}

Um Aussagen über klimatische Effekte unter Berücksichtigung der Szenarien treffen zu können, wurden Klimasimulationen durchgeführt. Bei der Modellerstellung wird darauf geachtet, dass die Ergebnisse der Simulationen mit den historischen Wetterbeobachtungen bestmöglich übereinstimmen. Die Berechnungsergebnisse werden dazu mit den räumlichen und zeitlichen Messreihen der Wetterdienste weltweit verglichen. Dies ermöglicht die Validierung der numerischen Modelle (Stocker u. a., 2013) sowie die Erstellung von Benchmarks zum Vergleich verschiedener Klimamodelle. 
In Abbildung 2.5 ist die Temperaturanomalie - also Differenz zwischen dem Messwert und dem langjährigen Mittelwert - sowohl von gemessenen Beobachtungen als auch simulierten Daten dargestellt. Als Referenzzeitraum wurde die vorindustrielle Zeit von 1850 bis 1900 gewählt und die globale mittlere Temperatur für diese Periode bestimmt. Die Ergebnisse der Wetterbeobachtungen zeigen, dass im Referenzzeitraum zwischen den gemessenen und gemittelten Temperaturen nur geringe Unterschiede vorhanden sind. Mit Zunahme der Industrialisierung und der damit verbundenen Erhöhung der Konzentration der Treibhausgase in der Atmosphäre stieg, mit einem Zeitversatz, die Temperatur der Erde ab ca. 1920 kontinuierlich an. Die historischen Daten, gewonnen aus Eisbohrkerne, zeigen für die vergangenen 800.000 Jahre eine maximale Konzentration von $\mathrm{CO}_{2}$ in der Atmosphäre von ca. 300 ppm (Lüthi u. a., 2008). Die weltweite Kohlendioxid-Konzentration ist laut dem Bundesumweltamt im Jahr 2019 auf einen Wert von 409,83 ppm gestiegen.

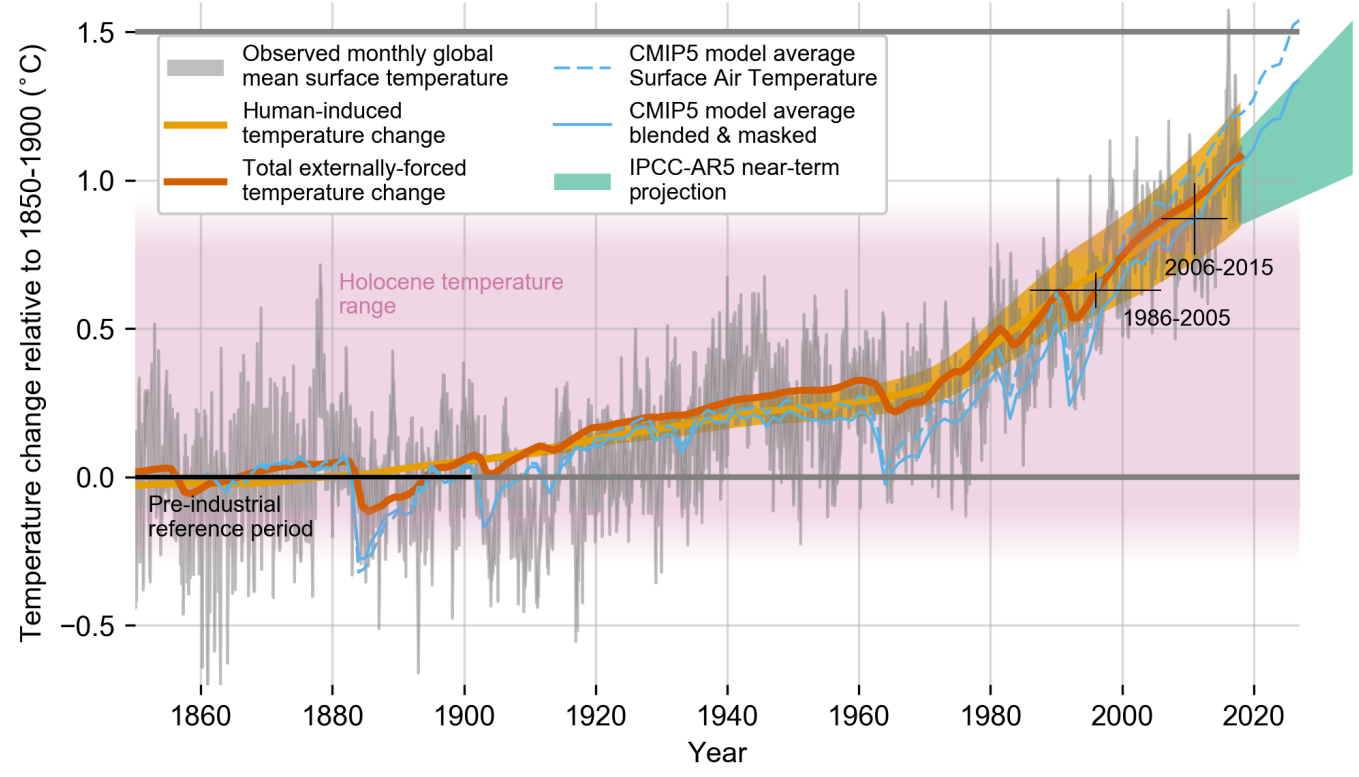

Abbildung 2.5

Globale Temperaturänderung relativ zum Mittelwert der Periode 1850 bis 1900, historische Werte (IPCC, 2013, Abbildung 1.2)

Projektionen des zukünftigen Klimas ergeben, dass zwischen 2030 und 2050 eine Erhöhung der globalen Temperatur der Erde um mehr als $1,50^{\circ} \mathrm{C}$ (Fehlerbalken) wahrscheinlich ist (Abbildung 2.6). Die Schätzung dieser Werte basiert auf der aktuellen Entwicklung der weltweiten Beobachtungen des Wetters. Aus der Darstellung kann weiterhin entnommen werden, dass in Abhängigkeit der emittierten Treibhausgase die langfristige Entwicklung der mittleren Oberflächentemperatur stark beeinflusst wird. Die Prozesse in der Atmosphäre sind träge, sodass eine sofortige Minderung des Ausstoßes aller Treibhausgase kurzfristig 


\section{Klimatische Bedingungen}

keine signifikante Abflachung der Temperaturanomalie bewirkt. Es muss davon ausgegangen werden, dass in den kommenden Dekaden weiterhin ungünstige Klimabedingungen vorherrschen und die Wetterbedingungen sich ungünstig für die Menschheit entwickeln (vgl. die Verläufe der Emissionsszenarien A1B und B1 in Abbildung 2.4).

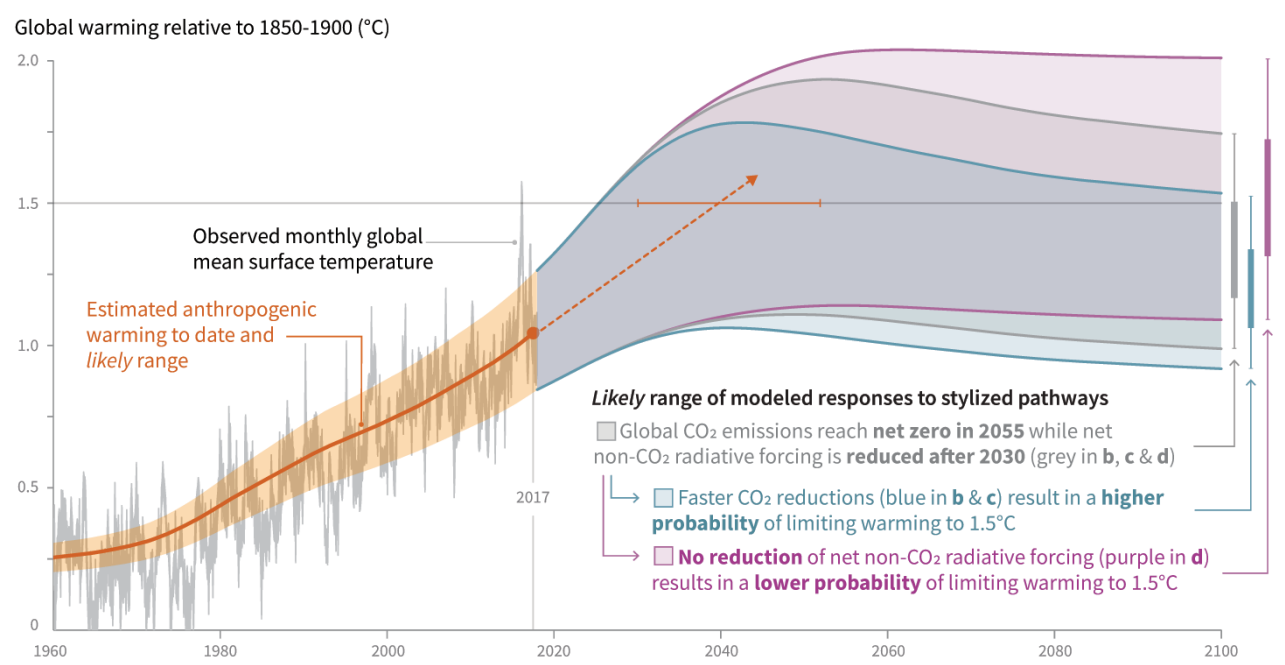

Abbildung 2.6

Globale Temperaturänderung relativ zum Mittelwert der Periode 1850 bis 1900, (IPCC, 2018, Abbildung SPM.1)

Die zukünftige Entwicklung des Klimas ist bedeutend für die politische und ökonomische Ausrichtung eines Landes. Zur Betrachtung von kleinräumigen Klimaunterschieden bedarf es Klimaprojektionen für hochaufgelöste Skalen. Durch Verringerung der räumlichen Auflösung und Anpassung von Modellparametern (statistisches und dynamisches Downsampling) können regionalisierte Klimamodelle erstellt werden. Diese Modelle können zusätzlich mit einem Boden-Vegetationsmodell oder einem Ozeanmodell gekoppelt werden. Grundsätzlich müssen regionale Klimamodelle in der Lage sein, kurzfristige Wetterereignisse ähnlich gut zu beschreiben, wie Modelle welche für die Wetterprognose verwendet werden. Bei Fehlern erfolgt auf Grund der langjährigen Berechnungszeiträume eine Akkumulation der Fehler und somit ergeben sich große Abweichungen zwischen realen Messungen und den simulierten Zuständen (Brasseur, Jacob und Schuck-Zöller, 2017, S. 27 ff.).

In der Vergangenheit wurde eine Vielzahl solcher regionalisierter Modelle generiert und die Entwicklung ist noch lange nicht abgeschlossen. Für die Risikoanalysen oder die Klimafolgenforschung in Deutschland wurden häufig die Modelle Climate Local Model (CLM) oder REgional MOdell (REMO) genutzt.

Die Güte dieser Klimamodelle wird an Beobachtungen des Wetters evaluiert. Dabei werden mit den Modellen Teile der historischen Beobachtungen des Wetters nachgerechnet. Die 
Eingangsdaten bilden sogenannte Reanalysedaten, welche aus Modellen der globalen Zirkulation unter Zuhilfenahme von täglichen Beobachtungen synthetisch generiert werden. Dieses Vorgehen ermöglicht eine realitätsnahe Beschreibung der Randparameter der Validierungsberechnungen (Brasseur, Jacob und Schuck-Zöller, 2017, S. 30). In einer Veröffentlichung von Schoetter u. a. konnte eine sehr gute Übereinstimmung zwischen den Berechnungen von Regionalmodellen (CLM und REMO) und den Beobachtungen der Lufttemperatur sowie der Windgeschwindigkeit im Bereich der Stadt Hamburg festgestellt werden (Schoetter u. a., 2012).

\subsection{Einfluss auf Straßenkonstruktionen}

In bisherigen Studien (Clauß, Kayser und Wellner, 2017; Kayser, 2007) wurden Untersuchungen, basierend auf dem Dimensionierungsverfahren der RDO Asphalt (2009), für exemplarisch ausgewählte Gebiete in Deutschland durchgeführt, wobei für jede untersuchte Region der Flächenmittelwert der Klimaparameter über ein größeres Gebiet bestimmt wurde. Eine genauere Betrachtung der Eingangswerte zeigt jedoch, dass die regionalen klimatischen Bedingungen jeder einzelnen Variante eine hohe Varianz aufweist (Mahlstein und Knutti, 2009). Betrachtet man die Daten der Klimafolgenanalysen für den Straßenbau des Projektes "Projizierter Klimawandel und Dimensionierung von Straßenbefestigungen" (Clauß, Kayser und Wellner, 2017), so zeigen sich signifikante Abweichungen der einzelnen Klimaparameter, z. B. der Temperaturen an den Netzknoten zu den Mittelwerten des Gebietes. Weitere für den Straßenkonstruktion wichtige klimatische Faktoren, wie die mittlere Globalstrahlung, Niederschlagsmenge oder Anzahl und Dauer von Hitzewellen, unterliegen ebenso diesen regional zum Teil stark unterschiedlichen Differenzen vom Mittelwert eines Bezugsgebietes. Der entwickelte Ansatz konnte für die Gebiete zeigen, dass ein erheblicher Unterschied in der Entwicklung von klimainduzierten Schädigungen an der Verkehrsinfrastruktur in Asphaltbauweise sehr wahrscheinlich ist (Abbildung 2.7). Auch haben die thermophysikalischen Materialparameter einen erheblichen Einfluss auf die quantitative Entwicklung der Schädigung.

Wistuba und Walther nutzen einen deutlich anderen Ansatz zur Prognose der Klimafolgen für die Verkehrsinfrastruktur (Wistuba und Walther, 2013). Die Autoren verwenden ein Verfahren, welches auf Stundenbasis den Ermüdungsstatus einer Straßenbefestigung aus Asphalt berechnet. In der Arbeit wurde die Entwicklung der Summe nach der Hypothese von Miner in Abhängigkeit von drei Emissionsszenarien für den Zeitraum 2001 bis 2030 dargestellt. Die Autoren gehen davon aus, dass der Ermüdungssatus nur wenig durch die Eingangsdaten beeinflußt wird. Weiterhin wurde von den Autoren festgestellt, dass die unterschiedlichen klimatischen Parameter zwar Effekte auf die Infrastruktur in Deutschland und Österreich haben, diese sich aber zukünftig nicht signifikant verändern werden. Anhand der Simulationsergebnisse (fünf Gebiete in Deutschland und ein Gebiet in Österreich) wurde auf die Entwicklung in Europa geschlossen. Die Autoren gehen davon aus, dass für 


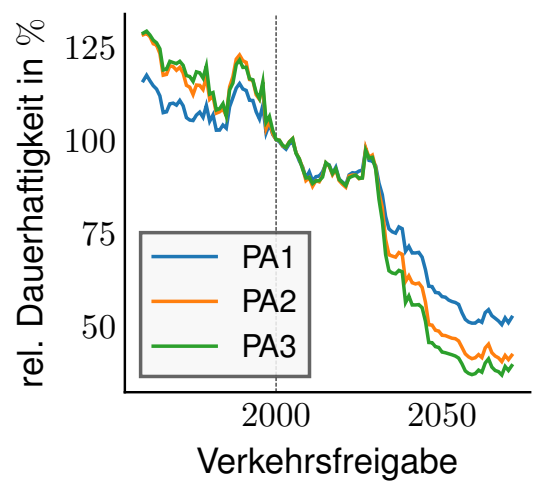

(a) Abhängigkeit von den Materialparametern, Raum Dresden

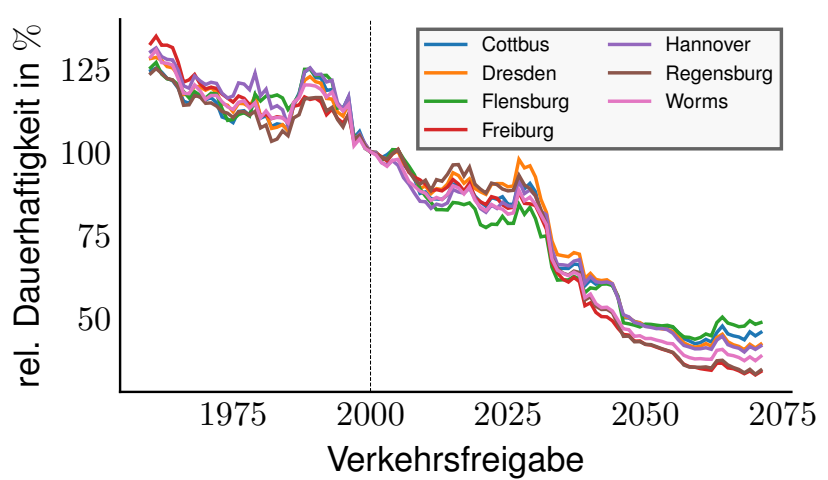

(b) Abhängigkeit vom Ort

Abbildung 2.7

Entwicklung der Dauerhaftigkeit von Straßenkonstruktionen in Abhängigkeit der Verkehrsfreigabe; Daten aus Clauß, Kayser und Wellner (2017)

Zentraleuropa keine Notwendigkeit einer Anpassung des Regelwerkes an die Anforderungen der Folgen des Klimawandels besteht.

In der internationalen Literatur findet man einige Hinweise auf den Einfluss des Klimawandels auf die Infrastruktur. Beispielhaft wird von Viola und Celauro empfohlen, dass die klimatischen Bedingungen bei der Auswahl der Baumaterialien im Prozess der Dimensionierung von Straßenbefestigungen in Italien zu berücksichtigen werden (Viola und Celauro, 2015). Eine quantitative Aussage über den Einfluss wurde nicht verfasst. In einer anderen Studie aus der USA konnte ein klarer Zusammenhang zwischen Klimawandel und den Folgen für die Verkehrsinfrastruktur abgeleitet werden (Gudipudi, Underwood und Zalghout, 2017). Die Autoren gehen davon aus, dass Straßenkonstruktionen stärker durch die Folgen des Klimawandels belastet werden und deshalb verfrüht ausfallen. Die Schädigungen im Bereich der Ermüdungsrissbildung sollen der Studie zufolge um 2\% bis $9 \%$ und die Spurrinnentiefe um $9 \%$ bis $40 \%$ zunehmen. In vielen Studien werden unterschiedlich starke Folgen angegeben. Die Autoren sind sich jedoch einig, dass ein Einfluss auf die Dauerhaftigkeit von Straßenkonstruktionen besteht und geeignete Anpassungsstrategien entwickelt werden müssen. 


\section{Dimensionierung von Straßenbefestigungen}

\subsection{Verfahrensbeschreibung}

In diesem Kapitel wird das genutzte rechnerische Prognoseverfahren unter Erläuterung der wesentlichen Parameter vorgestellt. Im Fokus stehen die relevanten Nachweise für die Bewertung der Auswirkungen des Klimawandels auf die Asphaltschichten. Anschließend werden Empfehlungen für notwendige Modifikationen des Verfahrens vorgeschlagen.

Die Schädigungen einer Straßenbefestigung in Asphaltbauweise durch den Verkehr können nach dem Verfahren der RDO Asphalt (2009) oder RSO Asphalt (2018) rechnerisch bestimmt werden. Dafür müssen analytische oder numerische Modelle der Konstruktionen erstellt und die Beanspruchungen auf Basis der Mehrschichtentheorie oder nach der Theorie der Finiten Elemente berechnet werden, wobei die Ergebnisse der Simulationen die Spannungen, Verformungen und Dehnungen innerhalb der Straßenbefestigung beschreiben. Die Beanspruchungszustände können anschließend mittels analytischer, empirischer oder statistischer Verfahren bewertet werden.

Bei der Verwendung der Mehrschichtentheorie wird die reale Straßenkonstruktion durch ein rotationssymmetrisches Modell idealisiert beschrieben, wobei der zu berechnende Straßenaufbau in Schichten unterteilt wird. Die Materialkennwerte innerhalb einer Subschicht werden als konstant festgelegt. Um die Temperaturabhängigkeit der Asphalte zu berücksichtigen, werden die Asphaltschichten weiterhin in Subschichten aufgegliedert. Der Radius $r$ des Modells wird als unendlich groß angenommen und der Untergrund wird zudem durch einen unendlich ausgedehnten Halbraum beschrieben. Bedingt durch die mathematische Formulierung dieses Systems werden nachfolgende Randbedingungen vorausgesetzt (Numrich, 2003, S. 48 ff; Neutag, 2005, S. 52-58):

- Der Schichtenverbund zwischen den einzelnen Subschichten kann nur als fest verbunden, reibungsfrei aufeinander gelagert oder als Teilverbund definiert werden.

- Die Einzelschichten müssen als dünne unendlich ausgebreitete Subschichten mit jeweils spezifischen Materialparametern definiert sein.

- Die eingetragene Belastung des Systems wirkt statisch.

- Das Stoffmodell wird als linear-elastisch angenommen. 


\section{Dimensionierung von Straßenbefestigungen}

- Die Parameter des Stoffmodells einer Subschicht sind homogen und isotrop.

Am Lehrstuhl für Stadtbauwesen und Straßenbau der Technischen Universität Dresden existiert ein nicht kommerzielles Programm auf Basis der Mehrschichtentheorie mit dem Namen Forschungsprogramm Straßenbau (ForProS). Diese Softwarelösung bietet unterschiedliche Berechnungs- und Materialmodelle und eine freie Programmierung. Im Rahmen dieser Arbeit wurde eine Programmierschnittstelle entwickelt, mit deren Hilfe eine skriptbasierte Modifikation der Eingangsdaten und eine Auswertung der Ergebnisse möglich ist. Die Verwendung eines Rechenkerns auf Basis der Finite Elemente Methode (FEM) hat Vorteile, jedoch verlängert sich die notwendige Zeit für eine Berechnung um ein Vielfaches. Eine Berechnung auf Basis der Mehrschichtentheorie dauert im Regelfall weniger als zwei Sekunden. Für eine Berechnung eines rotationssymmetrischen Straßenaufbaus mittels FEM z. B. in ABAQUS muss mehr als zwei Minuten gewartet werden. Der Umfang dieser Arbeit erfordert die Verwendung eines schnellen Berechnungsverfahrens, also kommt die Mehrschichtentheorie zur Anwendung.

Bevor Berechnungen durchgeführt werden können, müssen die Eingangsparameter für das rechnerische Dimensionierungsverfahren bestimmt bzw. definiert werden. Das Verfahren nach den RDO Asphalt (2009) definiert die notwendigen Arbeitsschritte (Abbildung 3.1, Auszug). Dem Schema ist zu entnehmen, dass zur Bewertung der Asphaltschichten vorab die Belastungen aus dem Verkehr und klimatischen Bedingungen definiert werden müssen. Auf die Eingangsdaten wird vertieft in Abschnitt 3.5 und Abschnitt 5 eingegangen. Der Konstruktionsaufbau wird durch die Festlegung der einzelnen Schichtdicken und der zugehörigen Materialien definiert. Die Parameter der verwendeten Materialmodelle werden im Regelfall im Labor mittels des Spaltzug-Schwellversuches bestimmt.

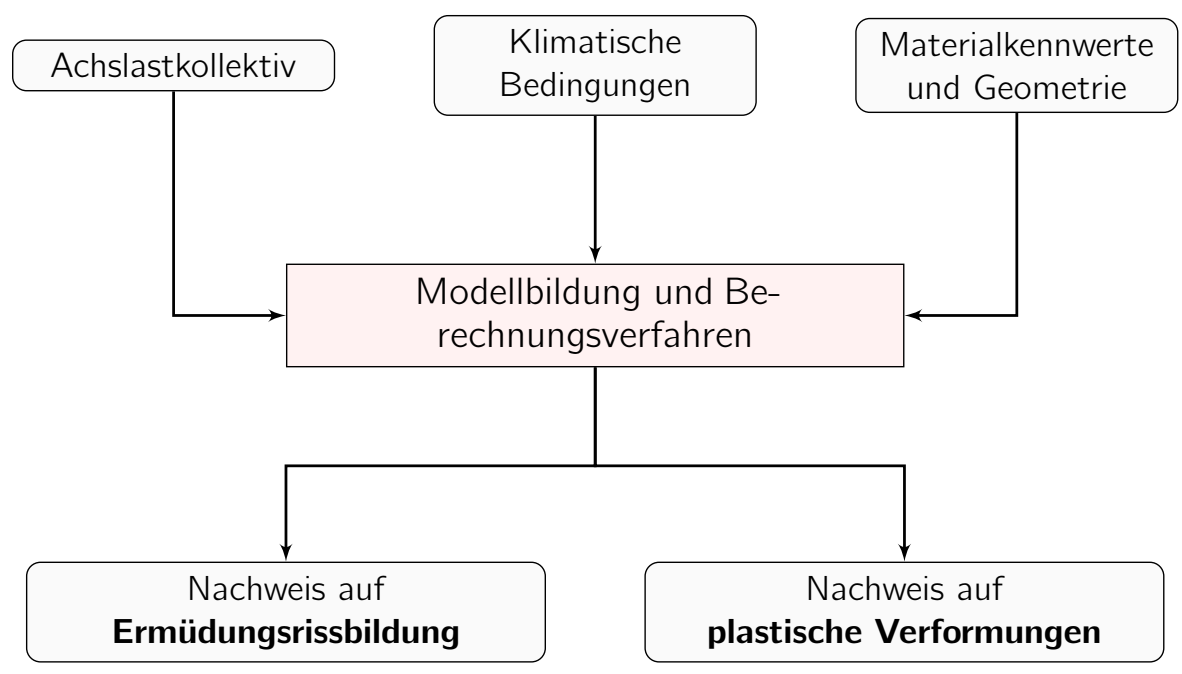

Abbildung 3.1

Ablaufschema über die Arbeitsschritte bei der rechnerischen Dimensionierung von Straßenbefestigungen, Auszug aus dem Gesamtverfahren nach den RDO Asphalt (2009) 
Das Ziel der rechnerischen Dimensionierung sind die Nachweise hinsichtlich Ermüdungsrissbildung und plastischer Verformung (Spurrinnenbildung). Die jeweiligen Methoden werden in den folgenden beiden Abschnitten näher erläutert, wobei die Nachweisführung an das Dimensionierungsverfahren nach den RDO Asphalt (2009) angelehnt ist.

\subsection{Nachweis auf Ermüdungsrissbildung}

Der Verkehr auf einer Straßenkonstruktion führt zu einer mechanischen Beanspruchung der einzelnen Schichten. Im Inneren der Straßenkonstruktion entstehen Spannungen, Dehnungen sowie Verformungen und bedingt durch diese Beanspruchungen können Risse im Asphalt entstehen. Das rechnerische Dimensionierungsverfahren nach den RDO Asphalt (2009) berücksichtigt diesen Schädigungsmechanismus. Nachfolgend sind die Grundlagen des Verfahrens dargestellt.

Die Beanspruchungen infolge des Verkehrs werden nach den Richtlinien durch sogenannte Achslastkollektive - bestehend aus repräsentativen Achslastklassen und deren zugehörigen Häufigkeiten - berücksichtigt. Für jede einzelne Belastung wird eine Simulation der mechanischen Beanspruchung durchgeführt. Je feiner die Aufteilung eines Achslastkollektives ist, desto genauer können die realen Beanspruchungszustände simuliert werden. Die Richtlinien (RDO Asphalt, 2009) definieren in der aktuellen Fassung drei unterschiedliche Achslastkollektive, wobei die Einteilung der Achslasten in Zwei-Tonnen-Klassen vorgenommen wurde.

Die unterschiedlichen klimatischen Bedingungen innerhalb Deutschlands haben Einfluss auf die thermischen Zustände der Straßenkonstruktionen. Im rechnerischen Dimensionierungsverfahren können unterschiedliche Temperaturzustände berücksichtigt werden (RDO Asphalt, 2009, S. 21). Die resultierenden Temperaturbedingungen in der Straßenbefestigung sind nach den Richtlinien nur abhängig von den Oberflächentemperaturen, stoffspezifische Anpassungsfaktoren werden nicht berücksichtigt. In der vorliegenden Arbeit wurden für die Berechnungen stets Simulationen zur Bestimmung des thermischen Verhaltens der gesamten Straßenkonstruktion durchgeführt und auf Basis der Daten statistische Auswertungen durchgeführt. Im Detail werden die Arbeitsschritte im Kapitel 4 und Kapitel 5 beschrieben.

Ergänzend zu Festlegung der Temperaturrandbedingungen und Verkehrlasten müssen die Parameter der Stoffmodelle für das Steifigkeits- und Ermüdungsverhalten der einzelnen Schichten der Straßenkonstruktion festgelegt werden. Die Parameter werden mittels des Spaltzug-Schwellversuches bestimmt. Dabei werden nicht strukturschädigende Multi-StageVersuche zur Bestimmung des temperatur- und frequenzabhängigen Steifigkeitsmoduls eingesetzt. Weiterhin wird das Ermüdungsverhalten bestimmt. Beide funktionalen Zusammenhänge werden im Dimensionierungsverfahren berücksichtigt.

Für jede Kombination aus Achslast und Temperaturbedingung wird eine mechanische Berechnung zur Bestimmung der Dehnungen innerhalb der Straßenbefestigung - mittels Mehrschichtentheorie oder FEM - durchgeführt. Basierend auf den Ergebnissen wird die 


\section{Dimensionierung von Straßenbefestigungen}

zulässige Anzahl an Lastwechseln $N_{\text {zul, } i}$ im Zustand $i$ bestimmt (Gleichung 3.1). Dieser Wert ist abhängig vom Ermüdungsverhalten des Asphaltes (Parameter a und b) und der berechneten Dehnung $\epsilon$. Bedingt durch die Unsicherheit des Verfahrens gegenüber zufälligen Variationen der Eingangsdaten wird die zulässige Lastwechselzahl mit dem Sicherheitsfaktor $S F \cdot F^{-1}$ multipliziert. Weiterhin muss für den Zustand $i$ die Anzahl an prognostizierten Achsübergängen $N_{\text {vor,i }}$ in Abhängigkeit der Häufigkeit des Auftretens eines bestimmten Temperaturprofils $\mathrm{H}_{\text {Klima }}$ und einer Achslastklasse $\mathrm{H}_{\text {Achslast }}$ berechnet werden (Gleichung 3.2).

$$
\begin{aligned}
N_{\text {zul }}, i & =\frac{S F}{F} \cdot a \cdot \epsilon^{b} \\
N_{\text {vor }, i} & =N \cdot H_{\text {Klima }} \cdot H_{\text {Achslast }}
\end{aligned}
$$

Mit der linearen Schädigungshypothese nach Miner (Miner, 1945) wurden die Beanspruchungen bewertet (Gleichung 3.3). Das Verhältnis von vorhandener zu zulässiger Lastwechselzahl ergibt den Schädigungsanteil oder Ermüdungsstatus im Zustand $i$. Die einzelnen Teilschädigungen wurden über alle Kombinationen aus Achslasten und Temperaturbedingungen akkumuliert. Die resultierende Gesamtschädigung zeigt das Potential gegenüber einer Rissbildung am Nachweispunkt. Die Hypothese nach Miner besagt, dass beim Erreichen des Grenzwertes die theoretische Lebensdauer (Makrorisskriteriums) ausgeschöpft ist. Dieser Zustand wird als Versagenskriterium innerhalb der Richtlinien definiert und in der vorliegenden Arbeit verwendet.

$$
\sum_{i=0}^{n} \frac{N_{\text {vor, } i}}{N_{z u l, i}} \leq 1
$$

\subsection{Nachweis auf Spurrinnenentwicklung}

Die Beanspruchungen in Straßenkonstruktionen, hervorgerufen durch Verkehrslasten, führen zu permanenten Verformungen der Asphaltschichten, welche als plastische Verformungen oder als Spurrinnen bezeichnet werden. Die Prognose von plastischen Verformungen ist ein Teil des Nachweisverfahrens in der vorliegenden Arbeit zur Beurteilung der Folgen des Klimawandels.

Im Regelwerk wurde ein Verfahren verankert, welches eine Abschätzung der Spurrinnengefährdung anhand der deviatorischen Vergleichsspannungen im Asphaltpaket ermöglicht (RDO Asphalt, 2009, Abschnitt 7.5.2). Jedoch unterliegt die Berechnungsweise einigen Einschränkungen. Unter anderem wird der Nachweis nur in der Asphaltdeck- und Asphaltbinderschicht oder bis in eine maximale Tiefe von $12 \mathrm{~cm}$ geführt. Weiterhin müssen nach den Berechnungen 
anhand der maximalen deviatorischen Vergleichsspannung Druck-Schwellversuche sowohl am Material der Asphaltdeck- als auch der Asphaltbinderschicht durchgeführt werden. Die labortechnischen Untersuchungen der Materialien liefern Hinweise auf die Anwendbarkeit der Asphalte. Im Verlauf der Belastung stellt sich im Regelfall ein Wendepunkt in der Impulskriechfunktion des Asphaltes ein. Ein Material mit einem sehr späten Wendepunkt gilt als besonders widerstandsfähig gegenüber plastischen Verformungen und sollte als Vorzugsvariante verbaut werden.

Bedingt durch die Verfahrensweise ist es nicht möglich, eine Aussage über die zeitliche Entwicklung der Spurrinne zu treffen. Aufgrund dieser Einschränkungen wurde die Methodik von Dragon und Reinhardt (2015) und Kayser, Reinhardt und Zeißler (2011) weiterentwickelt. Das Verfahren beruht darin, dass ein Stoffmodell aufgestellt wird, welches die Entwicklung der Spurrinnentiefe in Abhängigkeit der konkreten Materialparameter sowie der mechanischen Beanspruchungen in der Straßenbefestigung berücksichtigt.

Die Parameter des Stoffmodells zur Berechnung der Spurrinnenentwicklung können mittels Triaxial- oder einaxialen Druck-Schwellversuchen am schlanken Probekörper ermittelt werden. Dabei wird die Probe zyklisch belastet und die resultierende Verformung gemessen. Aus den Laboruntersuchungen (meist 30.000 Lastwechsel bei $10 \mathrm{~Hz}$ ) kann die Impulskriechfunktion in Abhängigkeit der Randbedingungen wie Temperatur und Spannungszustand anhand der Versuchsergebnisse nach Gleichung 3.6 bestimmt werden. Innerhalb der labortechnischen Bestimmung der Materialkennwerte werden die Versuchsbedingungen der Prüftemperatur sowie der Spannungszustände variiert. Anhand der Versuchsergebnisse können die Parameter $a_{0}$ und $a_{1}$ der Gleichung 3.4 und $b_{0}$ und $b_{1}$ der Gleichung 3.5 bestimmt werden. Diese definieren die Parameter des Stoffmodelles zur Berechnung des plastischen Verhaltens eines Asphaltes.

$$
\begin{aligned}
& a_{\text {axial }}=a_{0} \cdot\left|\epsilon_{e l, \text { anf }}\right|^{a_{1}} \\
& b_{\text {axial }}=b_{1} \cdot \ln \left|\epsilon_{e l, a n f}\right|+b_{0}
\end{aligned}
$$

Mittels Strukturanalysen werden die elastischen Dehnungen innerhalb der Straßenbefestigung infolge von definierten Beanspruchungszuständen berechnet. Jeder Beanspruchungszustand ist durch einen spezifischen Temperaturzustand der Straßenkonstruktion sowie einer konkreten Radlast definiert. Für die berechneten elastischen Dehnungen innerhalb der Straßenbefestigung werden die Parameter $a_{a x i a l}$ und $b_{a x i a l}$ bestimmt. Die resultierenden plastischen Verformungen können abschließend mit Gleichung 3.6 berechnet werden. Die Größe des Wertes ist dabei von der Anzahl der Überrollungen innerhalb eines Beanspruchungszustandes abhängig.

$$
\epsilon_{\text {plast }}=\mathrm{a}_{\text {axial }} \cdot \log (N+1)^{\mathrm{b}_{\text {axial }}}
$$

Unter realen Nutzungsbedingungen von Straßenkonstruktionen erfolgt eine zufällige Beanspruchung der Verkehrsfläche durch den Verkehr und die Temperaturbedingungen. Die 


\section{Dimensionierung von Straßenbefestigungen}

Reihenfolge von Fahrzeugen ist zufällig verteilt, die konkreten Temperaturbedingungen sind jedoch abhängig von der Jahreszeit. Einer sehr warmen sommerlichen Temperaturbelastung folgt nicht unmittelbar ein kaltes Winterereignis. Dies kann im Verfahren durch Temperaturintervalle berücksichtigt werden (Kayser, Reinhardt und Zeißler, 2011). Für jeden Temperaturzustand wird anhand der Achslastklassen eine Anzahl an Fahrzeugen in Abhängigkeit der Radlast definiert. Sowohl die Reihenfolge der Temperaturzustände als auch die Fahrzeuge werden zufällig gewählt. Für jeden Zustand wird die plastische Dehnung in der Straßenbefestigung berechnet und zur Gesamtverformung akkumuliert. Durch eine mehrfache Wiederholung der Berechnungen auf Grundlage von Zufallsprozessen ergeben sich für jeden Rechendurchlauf eine unterschiedliche Entwicklung der Spurrinnentiefe. Diese Entwicklungen können dann statistisch bewertet werden.

Das Berechnungsverfahren ist in Kayser, Reinhardt und Zeißler (2011) sowie Dragon und Reinhardt (2015) ausführlich dargestellt. Eine Anwendung des Verfahrens wurde z. B. in Clauß u. a. (2017) dokumentiert. Das beschriebene Verfahren wurde in dieser Arbeit zur Prognose der Entwicklung von Spurrinnentiefen in Abhängigkeit der projizierten, klimatischen Veränderungen verwendet.

\subsection{Hauptdehnungen}

Grundlage für die Durchführung von Prognose- und Dimensionierungsrechnungen bilden numerische Modelle der Straßenkonstruktion, mit deren Hilfe maßgebende Beanspruchungszustände bestimmt werden. Für die Nachweisführung auf Ermüdungsrissbildung und auf plastische Verformungen werden die berechneten Dehnungen in der Straßenbefestigung bewertet. Im Regelfall werden für die Nachweise auf Ermüdungsrissbildung die horizontalen Dehnungen an der Unterseite der Asphalttragschicht verwendet, da in diesem Bereich der Straßenbefestigung meist die größten horizontalen Dehnungen auftreten. Bei der Berechnung der plastischen Verformungen werden sowohl die vertiaklen als auch die horizontalen Dehnungen in der Straßenbefestigung berücksichtigt.

Der Aufbau der Straßenkonstruktion bewirkt, dass Beanspruchungen, hervorgerufen durch die Belastungen des Verkehrs, räumlich verteilt werden. Dies führt dazu, dass auch außerhalb der Radaufstandsfläche mechanische Beanspruchungen vorhanden sind. Mit analytischen oder numerischen Modellen kann an beliebigen Nachweispunkten in der Straßenkonstruktionen die Komponenten des ebenen Verzerrungstensors (Gleichung 3.7) berechnet werden. Dieser wird dabei durch drei Dehnungen in Richtung des Bezugskoordinatensystems und drei zusätzlichen Scherungen beschrieben.

$$
\epsilon=\left[\begin{array}{ccc}
\epsilon_{x} & \frac{1}{2} \gamma_{x y} & \frac{1}{2} \gamma_{x z} \\
\frac{1}{2} \gamma_{x y} & \epsilon_{y} & \frac{1}{2} \gamma_{y z} \\
\frac{1}{2} \gamma_{x z} & \frac{1}{2} \gamma_{y z} & \epsilon_{z}
\end{array}\right]
$$


Die Beanspruchungszustände innerhalb einer Straßenbefestigung variieren unter anderem in Abhängigkeit von der Verkehrsbelastung, der Fahrgeschwindigkeit, von der Aufstandsflächen des Rades und der Geometrie der einzelnen Schichten sowie deren Verbund. Es wird angenommen, dass der Nachweis auf Ermüdungsrissbildung auf Basis der Hypothese von Miner nur für gleiche oder sehr ähnliche Beanspruchungszustände gültig ist, jedoch konnte die Annahme in der Literatur nicht expliziet identifiziert werden.

Für den Nachweis auf Ermüdungsrissbildung innerhalb der gesamten Straßenbefestigung können nicht nur die horizontalen Dehnungen berücksichtigt sondern es müssen alle Komponenten des ebenen Verzerrungstensors bewertet werden. Durch eine Drehung des lokalen Koordinatensystems kann am Nachweispunkt ein Zustand ermittelt werden, an dem keine Scherungen am Element mehr vorhanden sind (Eigenvektor eines Verzerrungstensors). Die resultierenden drei Dehnungen in Richtung des lokalen Koordinatensystems werden als Hauptdehnungen bezeichnet (Gleichung 3.8). Die betragsmäßig größte Hauptdehnung wird nachfolgend verkürzt als Hauptdehnung bezeichnet und bei der Akkumulation der Einzelschädigungen verwendet.

$$
\bar{\epsilon}=\left[\begin{array}{ccc}
\epsilon_{1} & 0 & 0 \\
0 & \epsilon_{2} & 0 \\
0 & 0 & \epsilon_{3}
\end{array}\right]
$$

Die vielen Einflusskriterien auf eine Straßenbefestigung haben möglicherweise Auswirkungen auf die Akkumulationsprozesse der Einzelschädigungen. Es wird angenommen, dass die Dicke der einzelnen Schichten einer Straßenkonstruktionen sowie die Temperaturbedinungen während der Nutzungsphase die Ausrichtung der Hauptdehnungen beeinflussen. Um die Anwendbarkeit der Hauptdehnungen zur Nachweisführung nach der Hypothese von Miner zu prüfen, wurden in der vorliegenden Arbeit Serienrechnungen mit unterschiedlichen Geometrien eines Konstruktionsaufbaus bei gleichzeitiger Variation der Temperaturzustände durchgeführt. Die Serienrechnungen wurden mit einem kommerziellen Finite-ElementeProgramms (Abaqus) auf Basis eines 3D-Modells durchgeführt. Die Aufstandsfläche des Rades wurde als Kreisfläche simuliert. In Tabelle 3.1 sind die Eingangsdaten der multikriteriellen Analyse dargestellt. Durch Bildung des kartesischen Produktes der Eingangsparameter wurden alle möglichen Parameterkombinationen bestimmt und 729 Einzelrechnungen durchgeführt. In Abbildung 3.2 ist exemplarisch die Verteilung der Hauptdehnungen innerhalb der Asphaltbefestigung für zwei Beispiele dargestellt.

Tabelle 3.1

\section{Eingangsdaten der multikriteriellen Analyse}

\begin{tabular}{lcccc}
\hline \multicolumn{2}{c}{ Parameter } & Asphaltdeckschicht & Asphaltbinderschicht & Asphalttragschicht \\
\hline Dicke & $\mathrm{cm}$ & $2,4,6$ & $6,8,10$ & $14,18,22$ \\
E-Modul & $\mathrm{MPa}$ & & $5000,12500,20000$ & \\
\hline
\end{tabular}




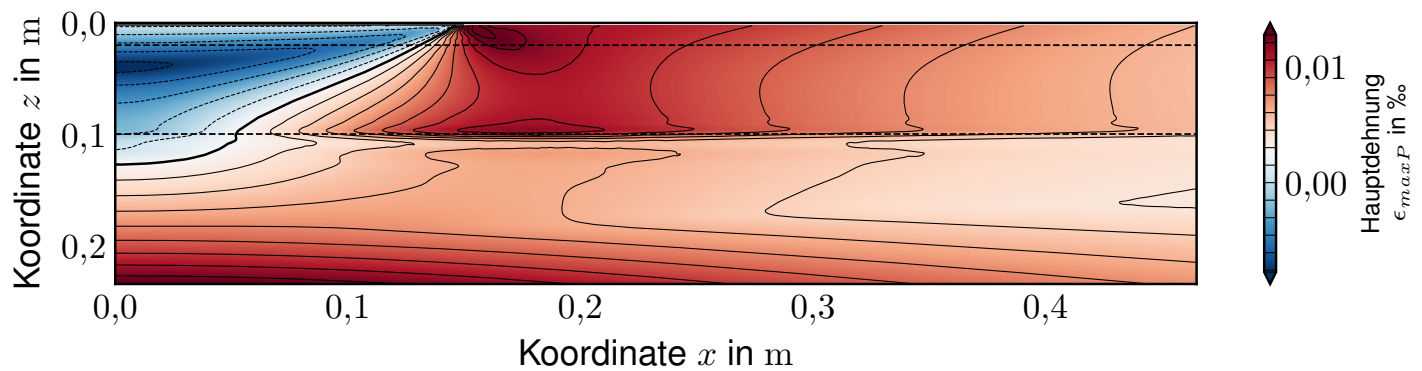

(a) Beispiel 1

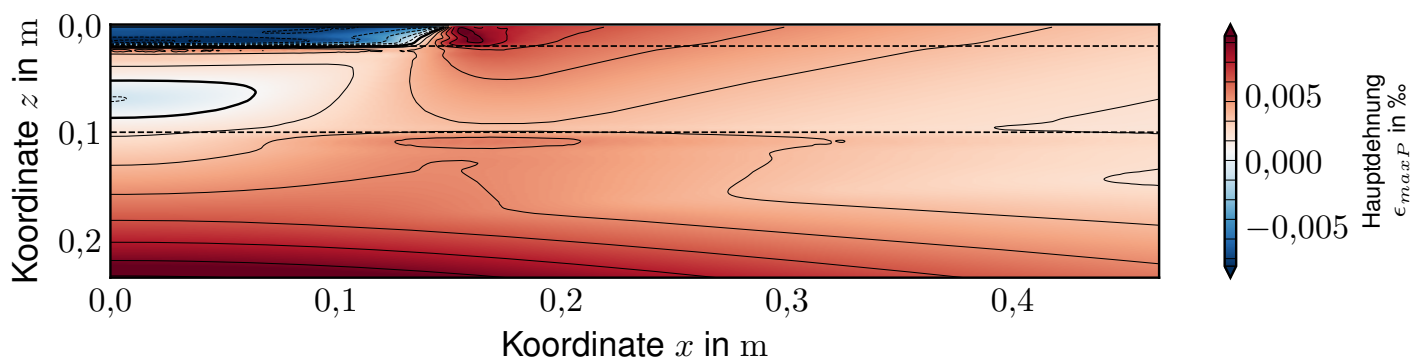

(b) Beispiel 2

Abbildung 3.2

Verteilung der Hauptdehnungen innerhalb einer Straßenkonstruktion, Beispiele aus Serienrechnungen

Aus den Ergebnissen aller Serienrechnunge wurden für alle Elemente anhand des Verzerrungstensors die Eigenvektoren (Richtung) und zugehörigen Hauptdehnungen (Wert) berechnet. Zusätzlich wurden die Winkel zwischen den Achsen des Bezugskoordinatensystems $(\vec{v})$ und dem lokalen Koordinatensystem des Eigenvektors der Hauptdehnungen ( $\vec{u}$ ) für jeden Nachweispunkt nach Gleichung 3.9 bestimmt.

$$
\cos (\varphi)=\frac{\vec{u} \cdot \vec{v}}{|\vec{u}| \cdot|\vec{v}|}
$$

In Abbildung 3.3 sind die Richtungen und Größen der Hauptdehnungen für eine untersuchte Geometrie exemplarisch dargestellt. Da die maximalen Beanspruchungen innerhalb der einzelnen Asphaltschichten in diesen Untersuchungen im Bereich der Lastachse auftreten, ist eine Reduktion auf eine zweidimensionale Darstellung möglich. In der Abbildung werden nicht die Hauptdehnungen aller Element der FE-Rechnungen dargestellt, sondern nur die Nachweispunkte mit der größten Hauptdehnung je Schicht der Straßenbefestigung. Für jede Geometrie ergeben sich 27 Kombinationen der zugeordneten Steifigkeiten, welche einzeln bewertet wurden. Die Simulationsergebnisse zeigen, dass bei gleicher Geometrie sich nur geringfügige Abweichungen bei der Richtung der Hauptdehnungen ergeben. Eine Anwend- 
barkeit der Hypothese von Miner unter Verwendung der Hauptdehnungen zur Akkumulation der Gesamtschädigung ist somit möglich.

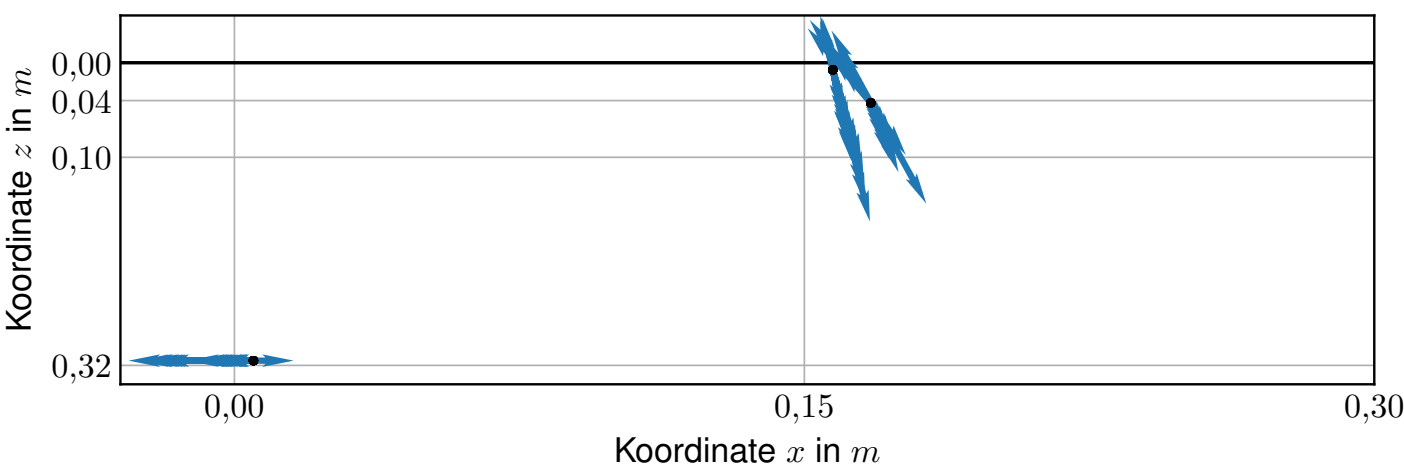

Abbildung 3.3

Lage der Nachweispunkte sowie Richtung und Größe (Länge der Pfeile) der Hauptdehnungen, dargestellt ist der Maximalwerte je Asphaltschichtin

Der Vergleich deutet weiterhin an, dass im Bereich der Asphaltdeckschicht ein erhöhtes Risiko gegenüber einer Ermüdungsrissbildung besteht. Dieser Sachverhalt wurde in der vorliegenden Arbeit für alle untersuchten Geometriekombinationen in Abhängigkeit der Steifigkeiten der Einzelschichten untersucht. Dazu wurde jede Asphaltschicht in neun Subschichten eingeteilt und der Nachweispunkt der entsprechenden Subschicht zugeordnet. Dies ermöglicht eine Bewertung der Lage unabhängig von der Dicke der einzelnen Schichten. In der Breite musste dies nicht vorgenommen werden, da die Einteilung des Netzes des FE-Modells für alle Varianten als gleich festgelegt wurde. Für jeden Rasterpunkt des FE-Netzes wurde weiterhin der Ermüdungsstatus unter der Verwendung der Ermüdungsfunktionen nach Tabelle 3.2 bestimmt. Zwischen den Varianten wurden einheitliche Randwerte für die Nachweisführung gewählt.

Tabelle 3.2

Ermüdungsfunktionen für die Nachweisführung

\begin{tabular}{lcll}
\hline Parameter & Asphaltdeckschicht & Asphaltbinderschicht & Asphalttragschicht \\
\hline a & 1 & 2 & 3 \\
b & 1 & 2 & 3 \\
\hline
\end{tabular}

Auf Basis der Analysen kann festgestellt werden, dass das höchste Risiko gegenüber der Entstehung von Ermüdungsrissen an der Unterseite der Asphalttragschicht zentrisch in der Lastachse besteht (Abbildung 3.4). Die Ergebnisse zeigen weiterhin, dass ein nicht unerhebliches Risiko auf Entstehung von Rissen innerhalb der Asphaltdeckschicht vorhanden ist. Bedingt durch die Verkehrsbeanspruchungen entstehen starke Dehnungen außerhalb der Aufstandsfläche des Rades. Dabei deutet sich an, dass ein Versagen nicht direkt an 


\section{Dimensionierung von Straßenbefestigungen}

der Oberseite der Asphaltdeckschicht entsteht, sondern in ca. 1/3 der Tiefe der Schicht. Sowohl die Ergebnisse aus Abbildung 3.3 als auch die Analysen über den Ort des Versagens (Abbildung 3.4) zeigen, dass Risse in diesem Bereich primär diagonal entstehen. Innerhalb der Asphaltbinderschicht konnte bei der Nachweisführung ein deutlich geringeres Risiko gegenüber einer Rissentstehung nachgewiesen werden. Die Lage ist nah am wahrscheinlichen Nachweispunkt in der Asphaltdeckschicht. Nachdem ein initialer Riss in der Asphaltdeckschicht entsteht, ist eine rasche Aufweitung in diesen Bereich sehr wahrscheinlich.

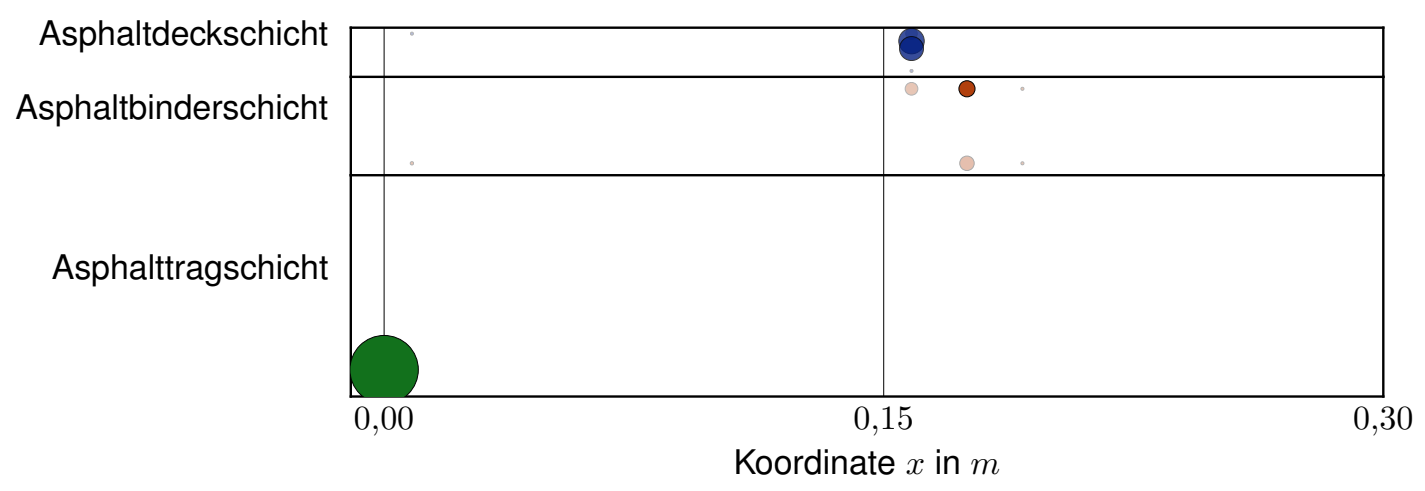

Abbildung 3.4

Ermüdungsstatus aller berechneten Varianten; der Durchmesser gibt die Summe aller Einzelberechnungen an

Möchte man innerhalb der gesamten Straßenbefestigung den Nachweis auf Ermüdungsrissbildung nach den RDO Asphalt (2009) führen, können nur gleiche Beanspruchungszustände zur Gesamtschädigung akkumuliert werden. Aus den Ergebnissen der durchgeführten Serienrechnungen konnte abgeleitet werden, dass eine Verwendung der Hauptdehnungen bei der Nachweisführung auf Ermüdungsrissbildung möglich ist. Die Bewertung aller Komponenten des Verzerrungstensors bietet zwei wesentliche Vorteile. Zum einen wirkt die maximale Hauptdehnung bei einer Geometrievariante stets in die gleiche oder eine ähnliche Richtung. Zum anderen wird durch die Bestimmung der Hauptdehnungen aus dem Verzerrungstensor die maximal auftretende Dehnung im Nachweisverfahren verwendet und somit eine Unterbewertung der Folgen der Belastungen aus dem Verkehr vermieden.

\subsection{Tagesganglinie der Verkehrsstärken}

Der Verkehr bildet die Hauptbelastung für Straßenbefestigungen und wird im Dimensionierungsverfahren nach den RDO Asphalt (2009) berücksichtigt. Für die Berechnung der Schädigungen der Straßenbefestigungen werden die Anzahl der Fahrzeuge in Abhängigkeit eines Achslastkollektives verwendet, wobei in den RDO Asphalt (2009) spezifische Verteilungen definiert sind. Diese wurden von Uhlig (Uhlig, 2019) analysiert und unter Berücksichtigung von Verkehrsmessungen erweitert. 
Wie bereits dargestellt, sind die resultierenden Dehnungen innerhalb der Straßenbefestigung stark von der Temperatur abhängig. Aus dem Alltag ist bekannt, dass vor allem am Tag eine hohe Anzahl an Fahrzeugen des Individual- als auch Schwerverkehrs fahren, wogegen sich in den Nachtstunden deutlich geringere Verkehrsstärken ergeben. Der Begriff der Verkehrsstärke beschreibt dabei die Anzahl an Fahrzeugen an einem Querschnitt während einer bestimmten Zeitspanne. Im Prognose- und Dimensionierungsverfarhren ist im Regelfall die Verkehrsstärke in einer Periode (meist jährlich oder gemittelt über 30 Jahre) als konstant definiert. In dieser Arbeit wurde der Einfluss dieser beiden Ansätze auf die Dauerhaftigkeit von Straßenbefestigung geprüft.

Entlang der deutschen Bundesstraßen und Autobahnen wurde ein dicht verteiltes Netz von Dauermesstellen zur automatisierten Erfassung des Verkehrsaufkommens installiert (Abbildung 3.5). Die Bundesanstalt für Straßenwesen (BASt) veröffentlicht regelmäßig die Messergebnisse dieser Dauermessstellen.

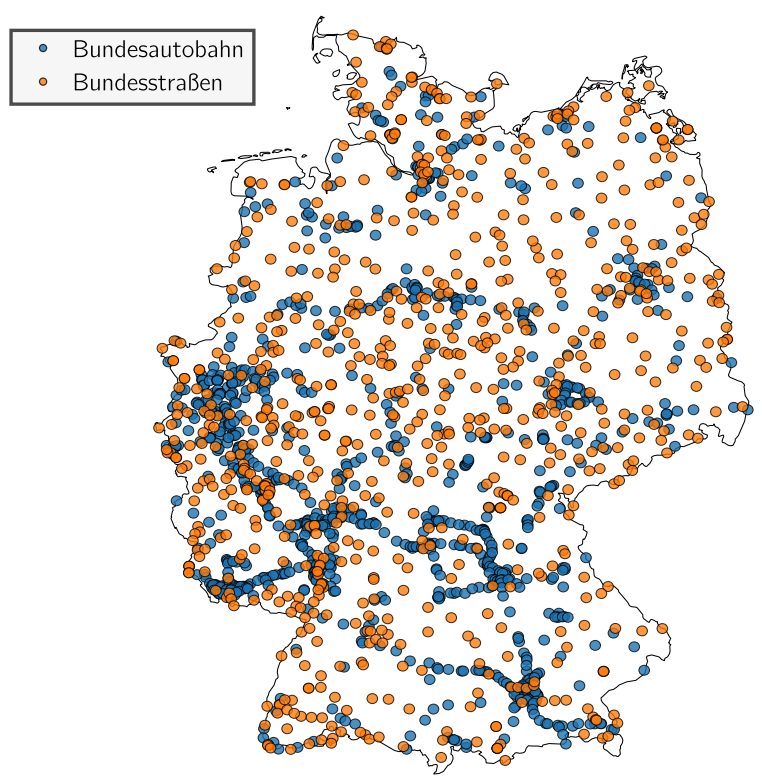

\section{Abbildung 3.5}

Übersicht über die Standorte der Dauermesstellen zur Erfassung des Verkehrsaufkommens innerhalb der Bundesrepublik Deutschland

Der öffentliche Datenbestand der Dauermessstellen umfasst allgemeine Angaben wie die Lage der Messstelle, als auch eine zeitabhängige Auflistung der einzelnen Fahrzeugklassen für den Zeitraum ab 2003. Die Daten aller Messstationen konnten mittels eines Auswertealgorithmus verarbeitet werden, wobei enthaltene Zeitlücken und Fehlmessungen berücksichtigt wurden. Für eine grundlegende Betrachtung wurden die Fahrzeugarten Pkw, Kleintransporter und Motorräder der Klasse KFZ und alle sonstigen Fahrzeuge der Klasse Schwerverkehr zugeordnet. Auf Basis der Klassifizierung der Daten wurde die Anzahl der Fahrzeuge in 


\section{Dimensionierung von Straßenbefestigungen}

Abhängigkeit von Tageszeit und Station berechnet (Abbildung 3.6). Weiterhin erfolgte eine Unterscheidung nach der Kategorie der Straße, also nach Bundesautobahnen und Bundesstraßen. In den Abbildungen sind die Einzelwerte aller Stationen als dünne Linien im Hintergrund dargestellt. Für jede Stunde wurde der Mittelwert aller Einzelstationen (Tabelle B.1) bestimmt und im Diagramm ergänzt. Auch ohne die Ermittlung der genauen Achslastverteilung können mit dieser Auswertung zeitliche Abhängigkeiten getrennt für KFZund Schwerlastverkehr analysiert werden. Diese zeitlichen Verteilungen können später auch mit vorhandenen, standardisierten Achslastverteilungen überlagert werden, um z.B. eine genauere Aufteilung des hier nur als Gesamtklasse erfassten Schwerverkehrs zu erreichen.

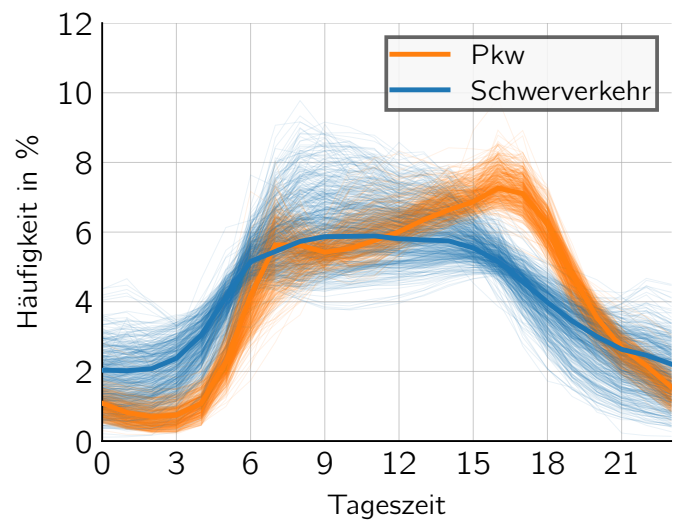

(a) Bundesautobahnen

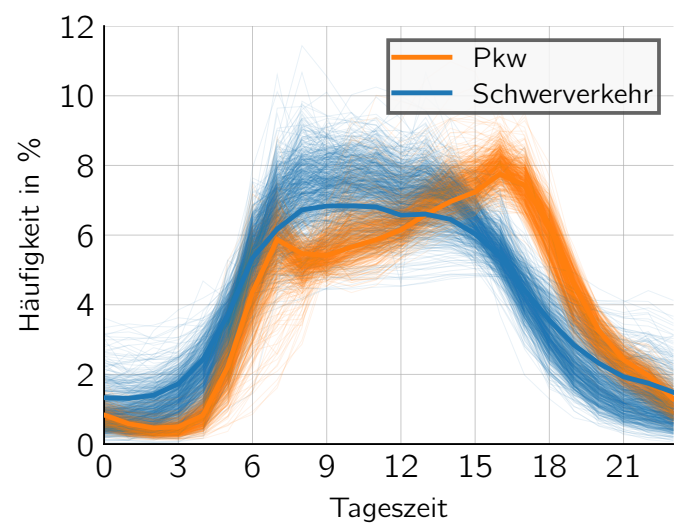

(b) Bundesstraßen

Abbildung 3.6

Relative Häufigkeit der Verkehrsbelastung in Abhängigkeit von der Tageszeit, Bundesdurchschnitt aller Messstationen, Zeitraum 2003 - 2016

Eine starke Abhängigkeit der Anzahl der Fahrzeuge von der Tageszeit konnte für beide Klassen ermittelt werden. In den Abend- und Nachtstunden sinkt die Häufigkeit des Auftretens der Fahrzeuge der Klasse KFZ und Schwerverkehr auf das gerinste Niveau und in den frühen Morgenstunden nimmt die Verkehrsstärke zu. Die Verteilung der Klasse Schwerverkehr hat den Höhepunkt am Vormittag und bleibt bis zum Nachmittag nahezu auf demselben hohen Niveau. In den Nachmittagsstunden sinkt die Verkehrsstärke allmählich gegen den Minimalwert, welcher in den Nachtstunden erreicht wird. Die Verteilung der Klasse KFZ sieht ähnlich aus. Die Daten zeigen auch hier eine deutliche Tag- und Nachtabhängigkeit. Die relative Anzahl der Fahrzeuge sinkt in den Nachtstunden deutlich ab. In den Tagstunden zeigen sich zwei Peaks durch den arbeitsbedingten Pendlerverkehr. Die maximale Verkehrsdichte wird, im Gegensatz zum Schwerverkehr, zwischen 16 Uhr und 19 Uhr erreicht.

In den RDO Asphalt (2009) wird eine lineare Verteilung der Verkehrsstärken über den Tag angenommen. Dies entspricht nicht den Beobachtungen des realen Verkehrsaufkommens. Die Häufigkeitsverteilungen der Temperaturzustände nach den RDO Asphalt (2009) wurden 
jedoch unabhängig von der Tageszeit definiert. Die Analyse der Oberflächentemperaturen einer Simulation des thermischen Verhaltens konnte bestätigen, dass wie zu erwarten ein erheblicher Unterschied auch bei den Temperaturzuständen in Abhängigkeit der Tageszeit vorhanden ist (Abbildung 3.7). Die Temperaturdifferenzen zwischen Tag und Nacht sind im Sommer am deutlichsten ausgeprägt, wobei Temperaturunterschiede von bis zu $20 \mathrm{~K}$ ermittelt wurden.

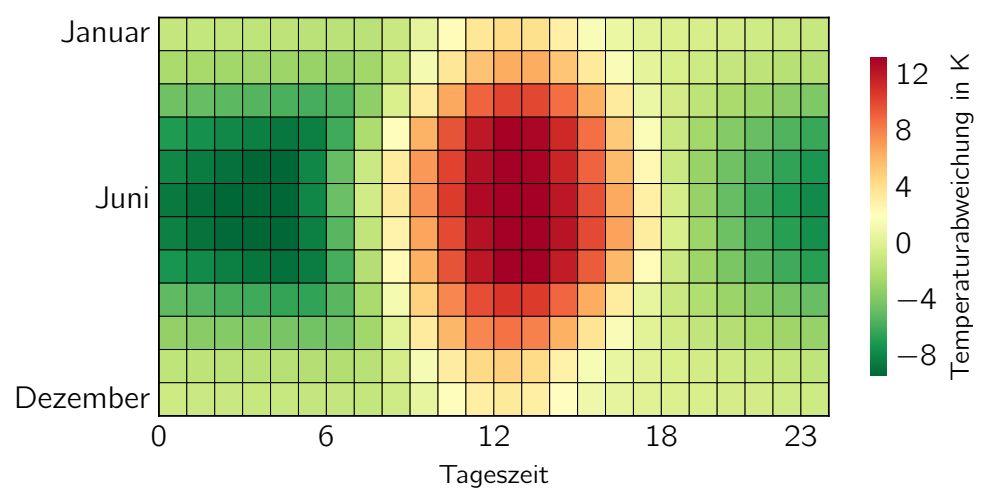

\section{Abbildung 3.7}

\section{Abweichung der Oberflächentemperaturen zum Monatsmittelwert in Abhängigkeit der Tages- zeit, Periode 2020 bis 2049 für das Gebiet Dresden}

Die Abängigkeit der Verkehrsstärken und Temperaturen in der Straßenbefestigung lässt vermuten, dass ein erheblicher Einfluss auf die Dauerhaftigkeit von Straßenbefestigungen vorhanden ist. Zur Überprüfung wurden in dieser Arbeit vergleichende Berechnungen durchgeführt (Abbildung 3.8). Zum einem wurde mit einer linearen Verteilung der Verkehrsstärken und zum anderen mit der Verteilungsfunktion der Verkehrsstärken für Bundesautobahnen gerechnet. Die Verteilung der Verkehrsstärken wurde mit dem Achslastkollektiv „BAB Fernverkehr" nach den RDO Asphalt (2009) überlagert und die Schädigungen infolge Ermüdungsrissbildung an der Unterseite der Asphalttragschicht bestimmt.

Die Prognoserechnungen zeigen, dass die zeitabhängige Berücksichtigung der Verkehrsstärke einen Einfluss auf die Ergebnisse haben. Für den Zeitraum mit der Verkehrsfreigabe ab 2020 wurde unter Verwendung der linearen Verteilung der Verkehrsstärken ein Ermüdungsstatus von 0,585 bestimmt. Die Berücksichtigung der zeitabhängigen Verteilung der Verkehrsstärken ergibt für denselben Zeitraum einen Wert von 0,604. Die Werte in diesem Beispiel unterscheiden sich nicht erheblich. Eine Berücksichtigung im Prognoseverfahren erscheint trotzdem sinnvoll, da die Überlagerung von hohen Temperaturen mit einer hohen Anzahl an Überrollungen durch den Verkehr wahrscheinlich die Entwicklung von Spurrinnen beeinflusst. Bedingt durch die Komplexität der Nachweisführung wurde dies in dieser Arbeit nicht berücksichtigt, eine Weiterentwicklung des Verfahrens unter Berücksichtigung der Verteilung der Verkehrsstärke wird empfohlen. 


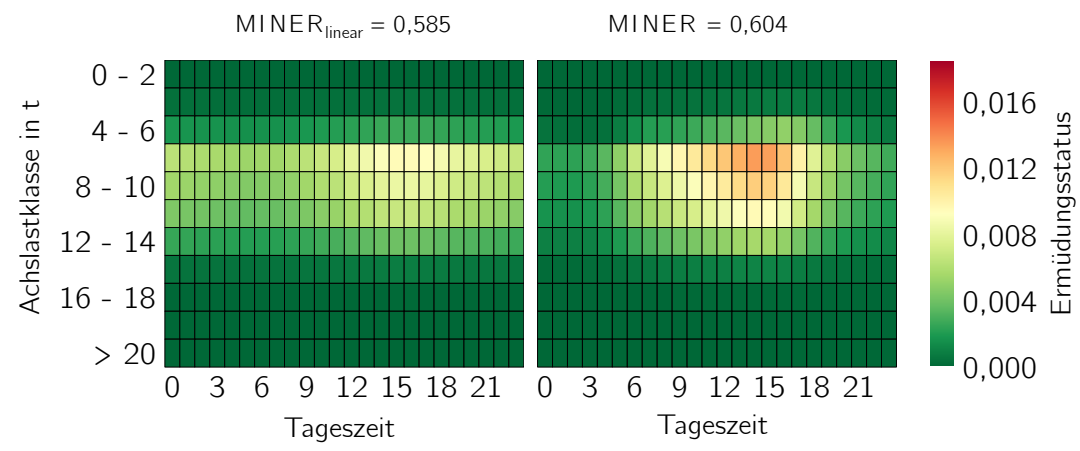

Abbildung 3.8

Ermüdungsstatus in Abhängigkeit der Tageszeit und der Achslastklasse, Raum Dresden, (links) linearen Verteilung der Verkehrsstärken (rechts) zeitabhängigen Verteilung der Verkehrsstärken

Die Unterteilung der Ergebnisse der Prognoserechnungen sowohl nach der Tageszeit als auch nach der Achslastklasse konnten bestätigen, dass leichte Fahrzeuge nur sehr geringe Schädigungsanteile an der Gesamtschädigung verursachen. Fahrzeuge mit Achslasten zwischen $6 \mathrm{t}$ und $14 \mathrm{t}$ verursachen den Hauptanteil an der Gesamtschädigung der Straßenkonstruktion.

Die Verwendung von unterschiedlichen Verteilungen der Verkehrsstärken haben einen direkten Einfluss auf die Ergebnisse der Prognoserechnungen. Im Falle linearer Verteilung der Verkehrsstärken entstehen relativ hohe Schädigungsanteile in den Nachtstunden. Die Verteilungen der Verkehrsstärke haben jedoch gezeigt, dass in diesem Zeitraum nur sehr wenige Fahrzeuge fahren, womit die hohen Schädigungsanteile in den Nachtstunden unrealistisch erscheinen. Die Verwendung des von der Verkehrsstärke abhängigen Achslastkollektives ergab, dass hauptsächlich Schädigungen im Tagesverlauf - in einem besonders hohen Maß in den Mittagsstunden - auftreten. Dies sind die Zeiträume, an denen ein sehr hoher Anteil des Schwerverkehrs die Infrastruktur nutzt. Das Simulationsergebnis ähnelt stark den gemessenen Verkehrsstärken.

Aus dieser Erkenntnis lassen sich Möglichkeiten ableiten, um die Dauerhaftigkeit von Straßenkonstruktionen durch eine aktive Verkehrssteuerung zu beeinflussen. Dabei könnten über eine Festlegung von Fahrzeiten die Verkehrsstärke kontrolliert beeinflusst werden. In Abbildung 3.9 sind drei mögliche Varianten vergleichend dargestellt. Die Basisvariante entspricht den Verkehrsstärke nach Abbildung 3.6, es wird also von den realen Verkehrsstärken ausgegangen. Bei den Vergleichsvarianten wurden die Verteilungen der Verkehrsstärken des Schwerverkehrs einmal um 6 Stunden nach vorn und um 6 Stunden zurück verschoben. Für jeden Zustand wurde der Ermüdungsstatus an der Unterseite der Asphalttragschicht mit sonst gleichen Eingangsdaten bestimmt. Der Versatz der Verteilung um 6 Stunden nach vorn führt zu einer Erhöhung des Schädigungspotenzials, daraufhin bewirkt ein früherer Startzeitpunkt eine deutliche Reduzierung des prognostizierten Ermüdungsstatus der Straßenbefestigung.

Die Anwendung des Verfahrens erfordert einen deutlich größeren Berechnungsaufwand im Vergleich zum Verfahren nach den Richtlinien. Für einen Nachweispunkt müssen im Falle 

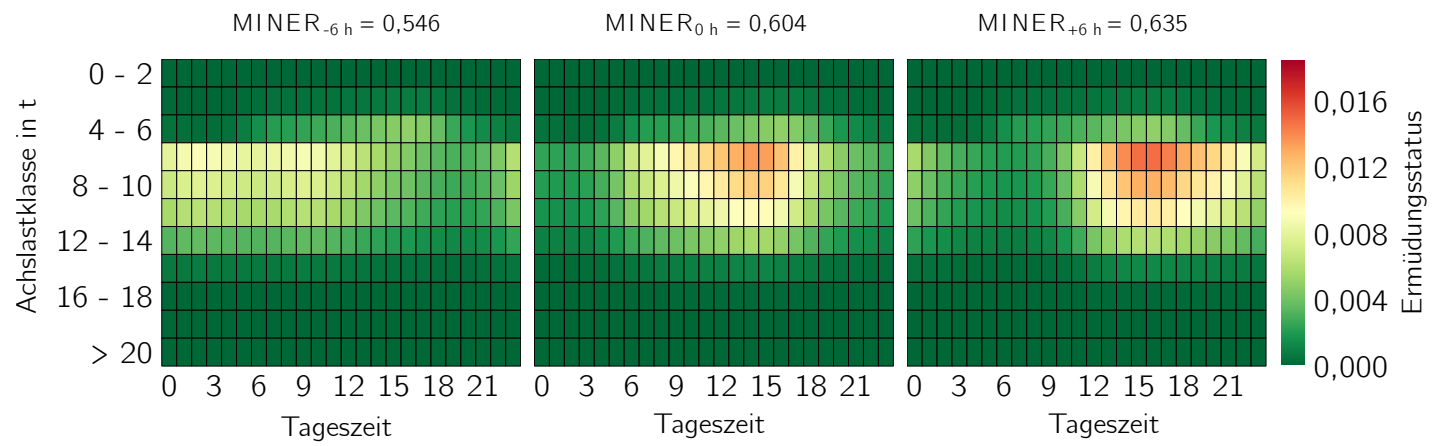

Abbildung 3.9

Potentialanalyse über den Einfluss einer Verkehrssteuerung für den Schwerverkehr, Versatz der Teilung der Verkehrsstärke (links) - 6 h, (mittel) 0 h, (rechts) $+6 \mathrm{~h}$

der Temperaturprofile und Achslastklassen nach den RDO Asphalt (2009) anstelle von 132 Einzelrechnungen bis zu 3168 Berechnungen durchgeführt werden. Bei Verwendung der normierten charakteristischen Temperaturprofilen (ncTs) ergibt sich eine notwendige Anzahl an Rechnungen von bis zu 41.184 Nachweisen. Das in dieser Arbeit vorgestellte Verfahren zur Bestimmung der Mittelwertfunktionen erfordert ca. 31.680 bis zu 118.800 Rechnungen für jeden Nachweispunkt. Unter Anwendung eines linear-elastischen Stoffmodells ist die Lösung dieser Aufgabe selbst mit herkömmlichen Rechnern mit einem vertretbaren Zeitaufwand möglich.

Die hier dargestellten Verteilungen der Verkehrsstärke zeigen einen deutlichen Einfluss auf die Berechnungen der Dauerhaftigkeit von Straßenbefestigung. Auf Grund der Komplexität dieser Berechnungen wurden keine Serienrechnungen zum Nachweis einer allgemeinen Anwendbarkeit durchgeführt. Es wird empfohlen, eine Integration des beschriebenen Verfahrens in die Richtlinien zu prüfen. Die Verwendung von zeitlich abhängigen Eingangsparametern aus Temperaturzuständen in Straßenbefestigungen und von der Verkehrsstärke abhängige Achslastkollektiven führt wahrscheinlich zu einer Steigerung der Güte des rechnerischen Dimensionierungs- und Prognoseverfahrens. 



\section{Simulation der thermischen Beanspruchungen}

\subsection{Allgemeines}

Mittels Simulationsrechnungen kann das thermische Verhalten von Objekten auf Basis von numerischen Verfahren abgeschätzt werden. Im spezifischen Fall von Straßenkonstruktionen ist es möglich, in Abhängigkeit der klimatischen Randbedingungen, die zeitlich nicht konstanten Temperaturbedingungen im Inneren zu berechnen. Die Eingangsparameter für die thermischen Simulationen können real gemessene Wetterdaten oder numerisch bestimmte Daten aus Klimamodellen sein. Die Anforderungen, der Modellaufbau und die Validierung der Simulationsergebnisse sind nachfolgend beschrieben.

Die Materialeigenschaften von Asphalten besitzen eine ausgeprägte Abhängigkeit von der Temperatur. Es ist also notwendig, die thermischen Prozesse innerhalb einer Straßenkonstruktion möglichst detailliert abzubilden. Langjährige Betrachtungen zur Energiebilanz des Gesamtsystems und Temperaturverteilungen in der Straße müssen möglichst genau die realen Prozesse abbilden. Die berechneten Temperaturverläufe in der Straßenkonstruktion werden als Eingangsgrößen für Dimensionierungsrechnungen verwendet, mit denen Aussagen über Dauerhaftigkeit von Straßenkonstruktion in Abhängigkeit unterschiedlichster Eingangsparameter wie thermophysikalische Materialparameter, Geometrie der Straßenkonstruktion oder klimatische Bedingungen abgeleitet werden sollen.

\subsection{Modellbeschreibung}

Im Projekt Untersuchung multifunktionaler Straßenbaumaterialien und Verbundwerkstoffe zur Nutzung solarer Energie und Verbesserung der Dauerhaftigkeit (Clauß u. a., 2019) wurde ein thermisches Modell entwickelt, welches die beschriebenen Anforderungen erfüllt und daher für sämtliche Betrachtungen in dieser Arbeit verwendet wurde. Bedingt durch die räumliche Ausdehnung der Straßenkonstruktion ist die Anwendung eines vereinfachten 1D-Modells denkbar und sinnvoll. Dieses thermische Simulationsmodell wurde auf Genauigkeit bei einer gleichzeitig hohen Performance optimiert. Dies ermöglicht die nachfolgenden detaillierten Betrachtungen auch über sehr lange Zeiträume. 


\section{Simulation der thermischen Beanspruchungen}

Die Aufbauten für Straßenkonstruktionen in Asphaltbauweise in Deutschland werden in den RStO 12 (2012) definiert und bestehen im Regelfall aus einem Asphaltpaket mit mehreren funktionalen Schichten, einer ungebundenen Frostschutzschicht und dem Untergrund. Der prinzipielle Aufbau ist schematisch in Abbildung 4.1 dargestellt. Zur Berechnung der Temperaturverteilung innerhalb der Straßenkonstruktion muss die Energiebilanz für das thermische Modell formuliert werden.

Die von der Sonne emittierte kurzwellige Globalstrahlung $\dot{Q}_{G}$ und die langwellige atmosphärische Gegenstrahlung $\dot{Q}_{\mathrm{AG}}$ bilden den eintretenden Strahlungswärmestrom und werden teilweise von der Fahrbahnoberfläche absorbiert. Ein Teil dieser Wärmeströme wird von der Straßenkonstruktion reflektiert. Aufgrund der Temperatur der Fahrbahnoberfläche wird der Strahlungswärmestrom $\dot{Q}_{\mathrm{FO}}$ emittiert. Zusätzlich wird durch die Luftbewegung zwischen der Umgebung und der Fahrbahnoberfläche ein konvektiver Wärmestrom $\dot{Q}_{K}$ übertragen. Die restliche aufgenommene Energie wird als Wärmestrom $\dot{Q}_{E}$ konduktiv in das Erdreich abgeleitet (Clauß u. a., 2019, S. 25 ff).
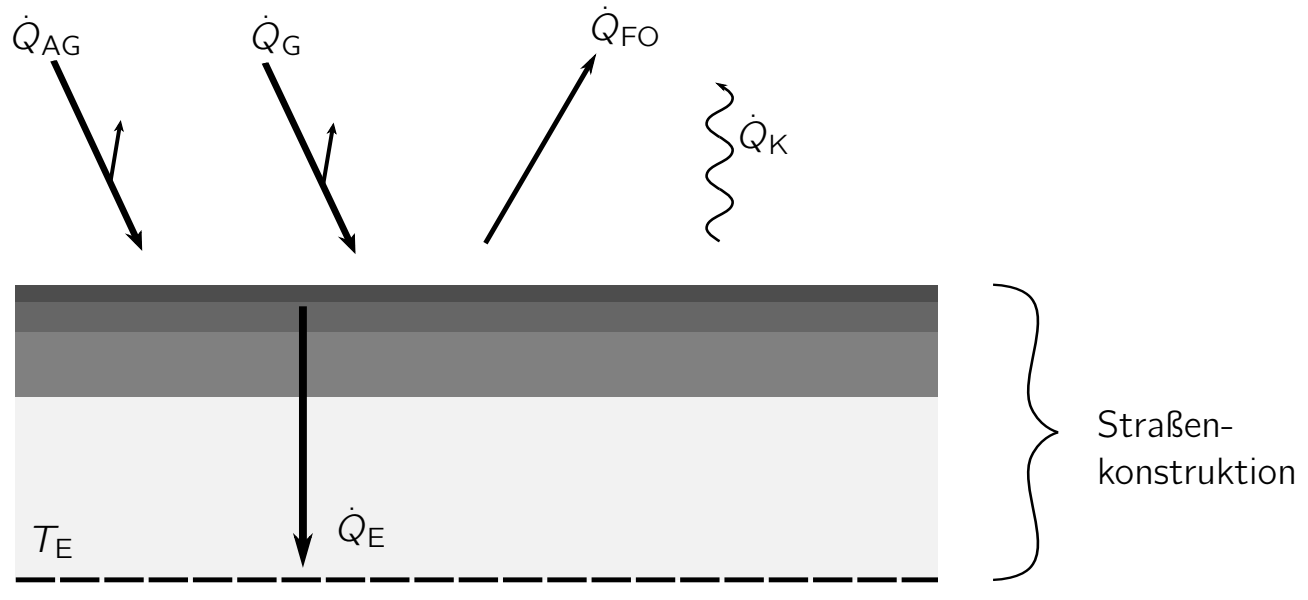

Abbildung 4.1

\section{Energiebilanz des thermischen Modells}

Zwischen den einzelnen Schichten der Straßenbefestigung erfolgt der Wärmetransport infolge von Wärmeleitung. Die Implementierung innerhalb des 1D-Schichtenmodells wird durch die Finite Volumen Methode (FVM) realisiert. Dabei wird angenommen, dass die Temperaturverteilung in Tiefenrichtung einer kontinuierlichen Änderung unterliegt, jedoch innerhalb einer Ebene der jeweiligen Schichten konstante Temperaturen auftreten. Diese Annahme ist aufgrund der im Verhältnis großen Breite und Länge zur Tiefe möglich, so dass Randeinflüsse vernachlässigt werden können. Die Betrachtung von zeitlich veränderlichen Randbedingungen bei instationären Simulationen erfordert die Berücksichtigung der Wärmespeicherfähigkeit aller Schichten und der zugehörigen spezifischen Materialien.

Zur Beschreibung der Wärmetransportvorgänge innerhalb der Straßenkonstruktion wird diese in finite Volumenelemente unterteilt (Abbildung 4.2). Für jedes Element werden die 
Koordinate $x_{i}$ und die Temperatur $T_{i}$ definiert. Die Energiebilanz für ein Volumenelement wird nach Alexiades und Solomon (1993) definiert als

$$
\begin{aligned}
& \frac{\mathrm{d} h_{i}}{\mathrm{~d} t}=\frac{1}{\varrho_{i} \Delta x_{i} A_{i}}\left(\dot{Q}_{i-1 / 2}-\dot{Q}_{i+1 / 2}\right) \\
& \text { mit: } \varrho \quad \text { Dichte } \\
& \Delta x_{i} \quad \text { Position des Mittelpunktes eines Zellvolumens } \\
& A_{i} \quad \text { Querschnittsfläche des Zellvolumens } \\
& \dot{Q}_{i \pm 1 / 2} \quad \text { Wärmestrom zu den angrenzenden Zellvolumen }
\end{aligned}
$$

mit den Wärmeströmen zu den angrenzenden Zellvolumen

$$
\begin{aligned}
\dot{Q}_{i-1 / 2}= & -\frac{T_{i}-T_{i-1}}{R_{i-1 / 2}} \\
\dot{Q}_{i+1 / 2}= & -\frac{T_{i+1}-T_{i}}{R_{i+1 / 2}} \\
\text { mit: } & T \quad \begin{array}{ll}
T & \text { Temperatur } \\
& R_{i \pm 1 / 2} \quad \text { Wärmewiderstände zwischen zwei Zellmittelpunkten }
\end{array}
\end{aligned}
$$

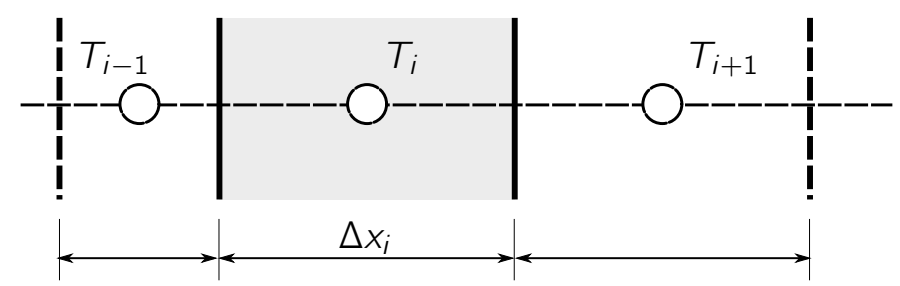

Abbildung 4.2

1D-Diskretisierung einer ebenen Platte mit der Finite-Volumen-Methode

Mit den Gleichungen

$$
\begin{aligned}
& R_{i-1 / 2}=\left(\frac{\Delta x_{i-1}}{2 \lambda_{i-1}}+\frac{\Delta x_{i}}{2 \lambda_{i}}\right) \cdot \frac{1}{A_{i-1 / 2}} \\
& R_{i+1 / 2}=\left(\frac{\Delta x_{i}}{2 \lambda_{i}}+\frac{\Delta x_{i+1}}{2 \lambda_{i+1}}\right) \cdot \frac{1}{A_{i+1 / 2}} \\
& \text { mit: } \Delta x_{i} \quad \text { Position des Mittelpunktes eines Zellvolumens } \\
& \lambda \quad \text { Wärmeleitfähigkeit } \\
& A_{i} \quad \text { Querschnittsfläche des Zellvolumens }
\end{aligned}
$$

können die beiden Wärmewiderstände $R$ zwischen zwei Zellmittelpunkten jeweils als Reihenschaltung der Widerstände zweier halber Zellen bestimmt werden. Dieses Vorgehen ermöglicht es, unter Berücksichtigung der Zustandsgleichung $h=h(p, T)$ (spezifische Ent- 


\section{Simulation der thermischen Beanspruchungen}

halpie $h$ und Temperatur $T$ ), temperaturabhängige Wärmekapazitäten zu nutzen (EnthalpieMethode nach Alexiades und Solomon, 1993). Damit lassen sich auch Phasenwechsel wie z. B. Frost-Tau-Wechselprozesse betrachten. Weiterhin können auch temperaturabhängige Stoffdatenfunktionen für die spezifische Wärmeleitfähigkeit $\lambda$ bei der Berechnung der Temperaturzustände berücksichtigt werden.

An der Ober- und Unterseite des thermischen Modells müssen Randbedingungen definiert werden. Auf die Oberfläche der Straßenkonstruktion treffen die kurzwellige Globalstrahlung der Sonne $\dot{Q}_{G}$ und die langwellige atmosphärische Gegenstrahlung $\dot{Q}_{\mathrm{AG}}$ ein. Ein Teil der Gesamtstrahlung wird an der Fahrbahnoberfläche reflektiert. Der Anteil der Reflexion wird auch als Albedo oder Reflexionsgrad mit dem Formelzeichen $\alpha$ bezeichnet. Die Größe des Albedos kann sowohl für die kurzwellige als auch für die langwellige Strahlung definiert werden. Bedingt durch die Temperatur der Fahrbahnoberfläche werden zusätzlich überwiegend langwellige Strahlen emittiert. Die ab- und zugeführten Wärmeströme an der Oberseite der Straßenkonstruktion können mittels der Gleichungen

$$
\begin{aligned}
& \dot{Q}_{\mathrm{FO}, \mathrm{zu}}=A \cdot\left(\dot{q}_{\mathrm{G}}+\dot{q}_{\mathrm{AG}}\right) \\
& \dot{Q}_{\mathrm{FO}, \mathrm{ab}}=A \cdot\left[\epsilon \sigma T^{4}+\left(1-\alpha_{\mathrm{G}}\right) \dot{q}_{\mathrm{G}}+\left(1-\alpha_{\mathrm{AG}}\right) \dot{q}_{\mathrm{AG}}\right] \\
& \text { mit: } \quad A_{i} \quad \text { Querschnittsfläche des Zellvolumens } \\
& \dot{q}_{\mathrm{G}} \quad \text { Wärmestromdichte der Globalstrahlung } \\
& \dot{q}_{\mathrm{AG}} \quad \text { Wärmestromdichte der athmosphärischen Gegenstrah- } \\
& \text { lung } \\
& \epsilon \quad \text { Emissionsgrad } \\
& \sigma \quad \text { Stefan-Boltzmann-Konstante } \\
& T \quad \text { Temperatur } \\
& \text { a } \quad \text { Albedo }
\end{aligned}
$$

beschrieben werden.

Bedingt durch den Effekt der Konvektion findet eine Wärmeübertragung zwischen der umgebenden Luft und der Fahrbahnoberfläche statt. Dieser Vorgang kann in die Komponenten der freien und erzwungenen Konvektion unterteilt werden. Der Anteil der Stoffübertragung infolge von Temperatur- und Dichteunterschieden wird als freie Konvektion bezeichnet, wohingegen bei der erzwungenen Konvektion eine äußere Strömung z. B. durch Wind aufgeprägt wird. Die Berechnung des konvektiven Wärmestromes $\dot{Q}_{K}$ wird durch das Newton'sche Wärmeübergangsgesetz

$$
\dot{Q}_{\mathrm{K}}=\alpha_{\mathrm{K}} A\left|\left(T_{\mathrm{OF}}-T_{\mathrm{L}}\right)\right|
$$

$\begin{array}{lll}\text { mit: } & \alpha_{K} & \text { Wärmeübergangskoeffizienten } \\ A & \text { Fläche der Fahrbahn } \\ & T_{\text {OF }} & \text { Temperatur der Fahrbahnoberfläche } \\ T_{\mathrm{L}} & \text { Temperatur der Luft }\end{array}$


beschrieben. Die Größe des Wärmestromes ist abhängig von Wärmeübergangskoeffizienten $\alpha_{K}$, der von vielen Randbedingungen, wie der Temperatur oder der Luftgeschwindigkeit, abhängig ist. Die Ermittlung des Wärmeübergangskoeffizienten kann mittels empirischer Gleichungen oder Kennzahlgleichungen erfolgen. Verfügbare Kennzahlgleichungen werden im Regelfall an einer Vielzahl von Experimenten validiert und sind somit Standard für Berechnungen des Wärmeüberganges.

Bei der Verwendung von Kennzahlgleichungen wird der konvektive Wärmeübergang zwischen der Fahrbahnoberfläche und der strömenden Luft mit der Nusselt-Zahl Nu beschrieben.

$$
\begin{aligned}
& N u=\frac{\alpha_{K} L}{\lambda} \\
& \text { mit: } \alpha_{K} \quad \text { Wärmeübergangskoeffizienten } \\
& L \quad \text { Charakteristische Länge } \\
& \lambda \quad \text { Wärmeleitfähigkeit }
\end{aligned}
$$

Der Zusammenhang zwischen dem Temperaturunterschied, der Strömungsgeschwindigkeit der Luft und der charakteristischen Länge der Fahrbahnoberfläche wird im Abschlussbericht des Forschungsprojektes (Clauß u. a., 2019) ausführlich beschrieben und für die nachfolgenden Berechnungen verwendet. Die Bestimmung des Wärmeübergangskoeffizienten erfolgt dabei mit Nusselt-Korrelationen aus dem VDI-Wärmeatlas (VDI e. V., 2013).

Im Rahmen des FuE-Projektes wurde das thermische Modell erstmals angewendet und anhand eines Testaufbaus validiert (Clauß u. a., 2019, S. 53 f).

\subsection{Eingangsparameter}

\subsubsection{Klimadaten}

Klimadaten bilden die Eingangsdaten für die Simulation des thermischen Verhaltens von Straßenkonstruktionen. In dieser Arbeit wurden die Eingangsdaten der Klimarechnungen auf Basis des Klimamodells REMO-UBA (Jacob, 2005a) mit den Emissionsszenarien C20 und $\mathrm{A} 1 \mathrm{~B}$ verwendet. Ziel der Untersuchungen ist es, eine Bewertung der thermischen Bedingungen sowohl für die Vergangenheit als auch für die Zukunft durchzuführen. Aus diesem Grund wurden die Klimadaten der Emissionsszenarien C20 und A1B kombiniert, was eine kontinuierliche Berechnung für den Zeitraum von 1960 bis 2100 ermöglicht.

Nachfolgend sind die einzelnen Arbeitsschritte zur Vorbereitung der Eingangsdaten aufgezeigt und abschließend werden die maßgebenden Klimaparameter am Beispiel des Stadtgebietes von Dresden dargestellt. 


\section{Simulation der thermischen Beanspruchungen}

Die Daten der Klimamodelle liegen in Form des Dateiformates netCDF vor. Dies ist ein spezifisches Dateiformat für den Austausch wissenschaftlicher Daten und die binären Datensätze sind in einer normierten Form abgespeichert. Für die thermischen Berechnungen der Temperaturen innerhalb von Straßenkonstruktionen wurden die Daten für die Lufttemperatur in einer Höhe von $2 \mathrm{~m}$ über dem Boden, die atmosphärische Gegenstrahlung, Globalstrahlung, Windgeschwindigkeit, Niederschlagsmenge und relative Luftfeuchtigkeit in einer sehr hohen zeitlichen Auflösung verwendet.

Die Ergebnisse der Klimarechnungen liegen in Stundenwerten vor. Im Betrachtungszeitraum von 1960 bis 2100 der Untersuchungen dieser Arbeit ergibt dies ca. 1,32 Millionen Datenpunkte. Das gewählte Klimamodell hat weiterhin eine sehr hohe räumliche Dichte an Knotenpunkten. Innerhalb des Gebietes der Bundesrepublik Deutschlands sind 4589 Knoten gleichmäßig verteilt und haben einen Rasterabstand von ca. $10 \mathrm{~km}$. Die Kombination aus den Zeitwerten, der räumlichen Knotenpunkte sowie der Klimaparameter ergibt eine Gesamtzahl von etwa 36,35 Milliarden Datenpunkten.

Bedingt durch die extrem hohe Dichte der verwendeten Klimadaten mussten diese vor Beginn der thermischen Simulationen aufbereitet werden. Ziel der Arbeit ist, die Folgen des Klimawandels auf die Verkehrsinfrastruktur in Deutschland zu beschreiben. Die Fläche in Deutschland ist in Bundesländer, Bezirke und Landkreise aufgeteilt. Die Bewertung der Klimafolgen auf Ebene der Bundesländer und Bezirke erscheint sehr grob. Somit erfolgte eine räumliche Mittelung der Eingangsdaten auf die Fläche der einzelnen Landkreise (Downsampling). Für diesen Vorgang wurden alle Knotenpunkte der Klimasimulation innerhalb eines Landkreises selektiert und in Abhängigkeit von dem Klimaparameter und der Zeit gemittelt. Die Anzahl der resultierenden Datensätze und der damit verbundenen Anzahl an Berechnungen konnte somit auf unter 500 Standorte reduziert werden. Die so synthetisch generierten Datensätze bilden die Eingangsdaten der nachfolgend dargestellten thermischen Simulationen.

In Abildung 4.3 sind exemplarisch drei klimatische Parameter aus der Klimasimulation für das Stadtgebiet von Dresden in Abhängigkeit von der Zeit und den Emissionsszenarien dargestellt. Die Stundenwerte der synthetischen Daten wurden aufbereitet und die jährlichen Mittelwerte berechnet. Anhand dieser Daten erfolgt die Bestimmung der gleitenden 30jährigen Mittelwerte (schwarze Kurven). Die gemittelte Lufttemperatur in einer Höhe von $2 \mathrm{~m}$ unterliegt einer starken jährlichen Schwankung (Abbildung 4.3a). Betrachtet man den Verlauf, so ist ersichtlich, dass die projizierten Temperaturen in der Vergangenheit unterhalb der Mittelwerte aus dem Jahr 2020 liegen. In den 1980er Jahren konnte eine geringfügig erhöhte Temperatur festgestellt werden, welche jedoch nach wenigen Jahren wieder abfiel. Seit Beginn der 2020er Jahre muss von einem kontinuierlichen Anstieg der Lufttemperatur ausgegangen werden. Die Daten des Klimamodells ergeben eine Erhöhung der jährlichen Mitteltemperatur für 2070 um über 2,50 K im Vergleich zu 2020.

Der Strahlungshaushalt der Erde wird sich zukünftig verändern. Die Werte der atmosphärischen Gegenstrahlung zeigen tendenziell ein ähnliches Verhalten wie die Lufttemperaturen (Abbildung 4.3b). Die Projektionen ergaben einen deutlichen Anstieg bis Ende des 


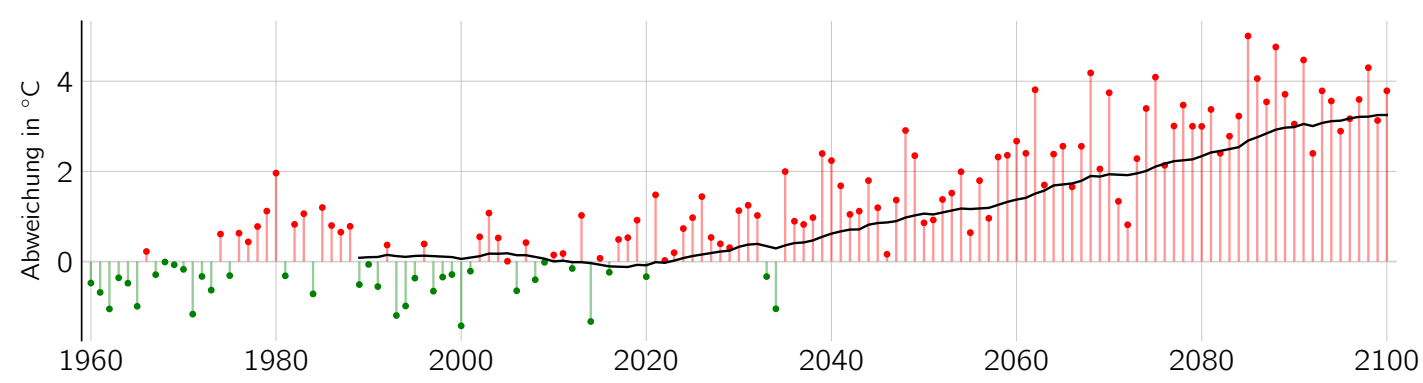

(a) Lufttemperatur in einer Höhe von $2 \mathrm{~m}$

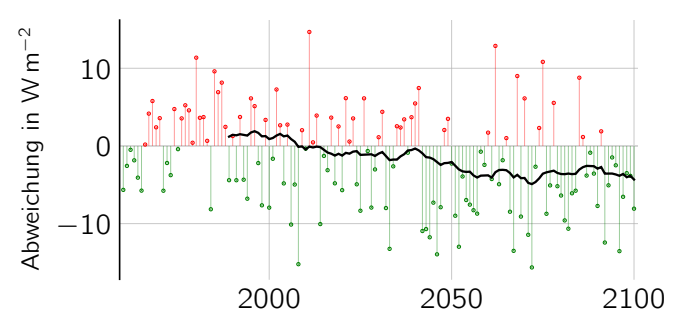

(b) Atmosphärische Gegenstrahlung

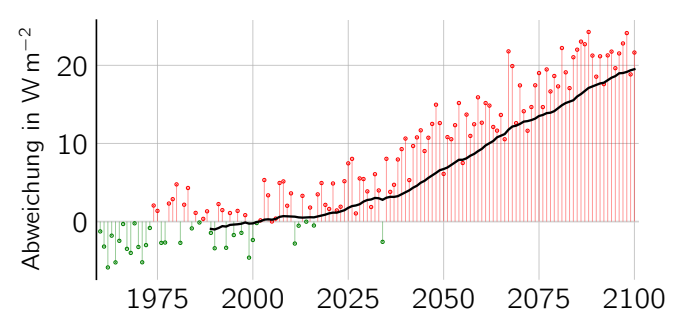

(c) Globalstrahlung

Abbildung 4.3

Abweichung der Klimaparameter bezogen auf den über die Fläche des Stadtgebietes von Dresden gemittelten Durchschnittswert der Periode 1960 bis 2020

Jahrtausends. Die Globalstrahlung innerhalb der Simulation unterlag starken jährlichen Schwankungen, wird jedoch zukünftig leicht abfallen (Abbildung 4.3c).

Die eingehenden Strahlungswärmeströme haben einen direkten Einfluss auf die Temperaturen in den Straßenkonstruktionen. Eine Erhöhung der Lufttemperatur bewirkt ebenso eine Erhöhung der resultierenden Temperaturan an der Fahrbahnoberfläche. Die Auswertungen der Klimadaten lässt somit vermuten, dass mit einer deutlichen Zunahme der Temperaturen in der Straßenkonstruktion zu rechnet ist. Die exakte Größe dieser Änderung kann aber nur mittels numerischer Berechnungen prognostiziert werden.

\subsubsection{Thermophysikalische Materialparameter und Geometrie}

Zur Durchführung der thermischen Simulationen werden Materialkennwerte für die einzelnen Schichten anhand der Messergebnisse aus dem FuE-Projekt (Clauß u. a., 2019) verwendet.

Nachfolgend werden die experimentellen Untersuchungen zur Bestimmung der thermischen Materialkennwerte kurz vorgestellt. Die Ermittlung der Raumdichten erfolgte an den Einzelproben nach dem Verfahren der TP Asphalt-StB Teil 6 (2007). Die Wärmeleitfähigkeit, Temperaturleitfähigkeit und die spezifische Wärmekapazität der einzelnen Asphaltproben wurden mit dem Messgerät ISOMET 2114 von Applied Precision Ltd. (Abbildung 4.4) an 


\section{Simulation der thermischen Beanspruchungen}

mehreren Positionen je Probe gemessen. Die Untersuchungen wurden unter konstanten Randbedingungen mit einer Temperatur der Probe von $20^{\circ} \mathrm{C}$ durchgeführt. Die Validierung des Messverfahrens erfolgte im Rahmen des FuE-Projektes.

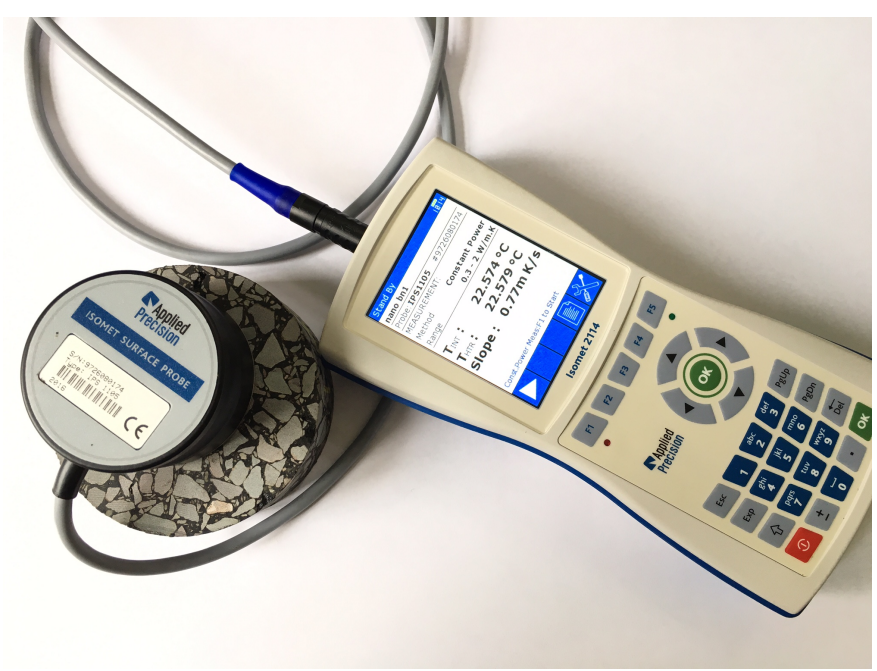

Abbildung 4.4

Wärmeleitfähigkeitsmessgerät ISOMET 2114 der Firma Applied Precision Ltd.

Die Einzelmessungen zeigen, dass die untersuchten Proben keine homogenen Materialkennwerte aufweisen. Asphalt ist ein Stoffgemisch aus Gestein, Bitumen und Luftporen, welche die thermophysikalischen Materialparameter lokal beeinflussen. Die Wärmestromdichte innerhalb einer Asphaltschicht ist somit nicht über die gesamte Fläche konstant. Zur realitätskonformen Beschreibung der Wärmetransportvorgänge müssten diese Aspekte mittels eines 3D-Modells unter Berücksichtigung der Mikrostruktur umgesetzt werden. Dies ist denkbar, führt jedoch zu einer erheblichen Steigerung der Berechnungszeiten. Bei thermischen Simulationen mit langen Betrachtungszeiträumen ist dies nicht praktikabel. Für die Anwendung eines 1D-Modells wurden Einzelmessungen aufbereitet und in Abhängigkeit des Materials gemittelt um Effektivgrößen zu erhalten. Die so bestimmten Materialkennwerte sind in Tabelle 4.1 zusammengestellt.

Tabelle 4.1

Thermophysikalische Materialparameter der verwendeten Asphalte

\begin{tabular}{|c|c|c|c|c|}
\hline Material & $\begin{array}{l}\text { Raumdichte } \\
\rho\left[\frac{g}{c m^{3}}\right]\end{array}$ & $\begin{array}{l}\text { Wärmeleit- } \\
\text { fähigkeit } \\
\lambda\left[\frac{W}{m \cdot k}\right]\end{array}$ & $\begin{array}{l}\text { spezifische } \\
\text { Wärmekapazität } \\
c_{p}\left[\frac{J}{\mathrm{~kg} \cdot \mathrm{K}}\right]\end{array}$ & $\begin{array}{l}\text { Temperatur- } \\
\text { leitfähigkeit } \\
a \cdot 10^{-6}\left[\frac{\mathrm{m}^{2}}{\mathrm{~s}}\right]\end{array}$ \\
\hline Asphaltdeckschicht & 2,53 & 1,46 & 654,13 & 0,88 \\
\hline Asphaltbinderschicht & 2,56 & 1,64 & 675,85 & 0,95 \\
\hline Asphalttragschicht & 2,56 & 1,33 & 648,61 & 0,80 \\
\hline
\end{tabular}




\subsection{Simulationsergebnisse}

Nachfolgend werden die Ergebnisse der Simulationen der thermischen Zustände von Straßenkonstruktionen grafisch dargestellt und erläutert. Die aufgeführten Zeitangaben entsprechen jeweils einem 30-jährigen Zeitraum, dessen Beginn die Nutzung der Straßenkonstruktion, also den Zeitpunkt der Verkehrsfreigabe, darstellt. Die Analysen von Zeiträumen erfolgen jeweils an dem gleitenden Durchschnitt über eine 30-jährige Periode und die Ergebnisse werden nachfolgend sowohl als relative als auch absolute Änderungen verwendet.

Weiterhin werden die Analysen nur für die Asphaltdeckschicht und Asphalttragschicht durchgeführt. Diese sind im Allgemeinen die Schichten, in denen Schädigungen an der Straßenkonstruktion am wahrscheinlichsten sind. Die zu analysierenden Parameter wie z. B. die mittlere Temperatur in der Asphaltdeckschicht werden nicht punktuell an der Oberoder Unterseite der Schicht bestimmt, sondern es werden jeweils die Temperaturzustände innerhalb der gesamten Schicht berücksichtigt.

Die im nachfolgenden dargestellten Diagramme und weiteren Parameter sind in einer detaillierteren Form dem Anhang A beigefügt.

Für den Schichtenaufbau wurde nach den RStO 12 (2012, Tafel 1) die Variante Asphalttragschicht auf Frostschutzschicht mit den Schichtstärken Asphaltdeckschicht $4 \mathrm{~cm}$, Asphaltbinderschicht $8 \mathrm{~cm}$. Asphalttragschicht $22 \mathrm{~cm}$, Frostschutzschicht $56 \mathrm{~cm}$ und einer Gesamttiefe von 10 m gewählt.

\subsubsection{Maximaltemperaturen}

Die maximalen Temperaturen innerhalb der untersuchten Straßenkonstruktion bilden einen Indikator für die Anfälligkeit einer Asphaltbefestigung gegenüber klimatischen Veränderungen. Dies begründet sich auch aus der starken Abhängigkeit der Materialeigenschaften von der Temperatur. Aus Laboruntersuchungen geht hervor, dass im hohen Temperaturbereich, also bei Temperaturen größer als $50^{\circ} \mathrm{C}$. Asphalte nur noch ein sehr geringes Steifigkeitsmodul aufweisen. Somit entstehen unter Belastungen aus dem Verkehr sehr hohe resultierende Dehnungen innerhalb von Straßenkonstruktionen, welche unter dem Aspekt der Ermüdungsrissbildung nach der Hypothese von Miner und den bleibenden plastischen Deformationen zu einer erhöhten Schädigung der Straßenkonstruktion führen.

Die Abbildung 4.5 zeigt eine starke Abhängigkeit der Temperaturen in der Straßenbefestigung von den lokalen Klimabedingungen. Sowohl die Verteilungen der Temperaturen in der Asphaltdeckschicht als auch innerhalb der Asphalttragschicht zeigen ein deutliches Nord-Süd-Gefälle. Straßenkonstruktion, welche im Bereich der Nord- und Ostsee errichtet werden, unterliegen aufgrund der lokalen klimatischen Bedingungen einer geringeren thermischen Belastung. Je südlicher sich eine Straßenkonstruktion innerhalb des Bundesgebietes befindet, desto höher ist auch die thermische Beanspruchung, welche jedoch nicht homogen verteilt ist. Regionale Bedingungen wie Gebirge haben einen erheblichen Einfluss auf die 


\section{Simulation der thermischen Beanspruchungen}

Temperaturzustände innerhalb einer Straßenkonstruktion. Bedingt durch den Prozess der Mittelung der Klimaparameter auf die Fläche der Kreise (vgl. Kapitel 4.3) werden sehr kleinräumige Klimaparameter geglättet, sodass die realen Bedingungen von den hier gezeigten Mittelwerten abweichen können.

Die zukünftigen klimatischen Bedingungen haben weiterhin einen direkten Einfluss auf die resultierenden Temperaturverteilungen innerhalb der Straßenkonstruktionen. Die maximalen Temperaturen in der Asphaltdeckschicht werden zukünftig kontinuierlich steigen (Abbildung 4.5a). Die gemittelten Maximalwerte der Temperaturen innerhalb der Asphaltdeckschicht liegen für die Zeiträume 1960 und 2020 nahezu konstant bei ca. $57,50^{\circ} \mathrm{C}$. Mit Zunahme der Effekte des projizierten Klimawandels steigt auch die maximale Temperatur innerhalb der Asphaltdeckschicht auf einen mittleren Wert von ca. $60,40{ }^{\circ} \mathrm{C}$ im Jahr 2070. Die genannten Werte entsprechen dabei dem gemittelten Wert für das Bundesgebiet. Die absolute maximale Temperatur innerhalb der Straßenkonstruktion wurde mit ca. $68^{\circ} \mathrm{C}$ simuliert. Aus der räumlichen Verteilung ist zu entnehmen, dass der Großraum München, Zentraldeutschland und die Gebiete im Südwesten einer deutlichen Steigerung der thermischen Belastung unterliegen.

Die Beeinflussung der thermischen Zustände der Asphaltdeckschicht kann qualitativ auch für die Asphalttragschicht nachgewiesen werden (Abbildung 4.5b). Bedingt durch die tiefere Lage der Asphalttragschicht innerhalb der Straßenkonstruktion sind die absoluten Maximalwerte der Temperatur geringer als bei den Asphaltdeckschichten. Die Aussagen zu zeitlichen und räumlichen Veränderungen treffen jedoch ebenso für die Asphalttragschicht zu. 

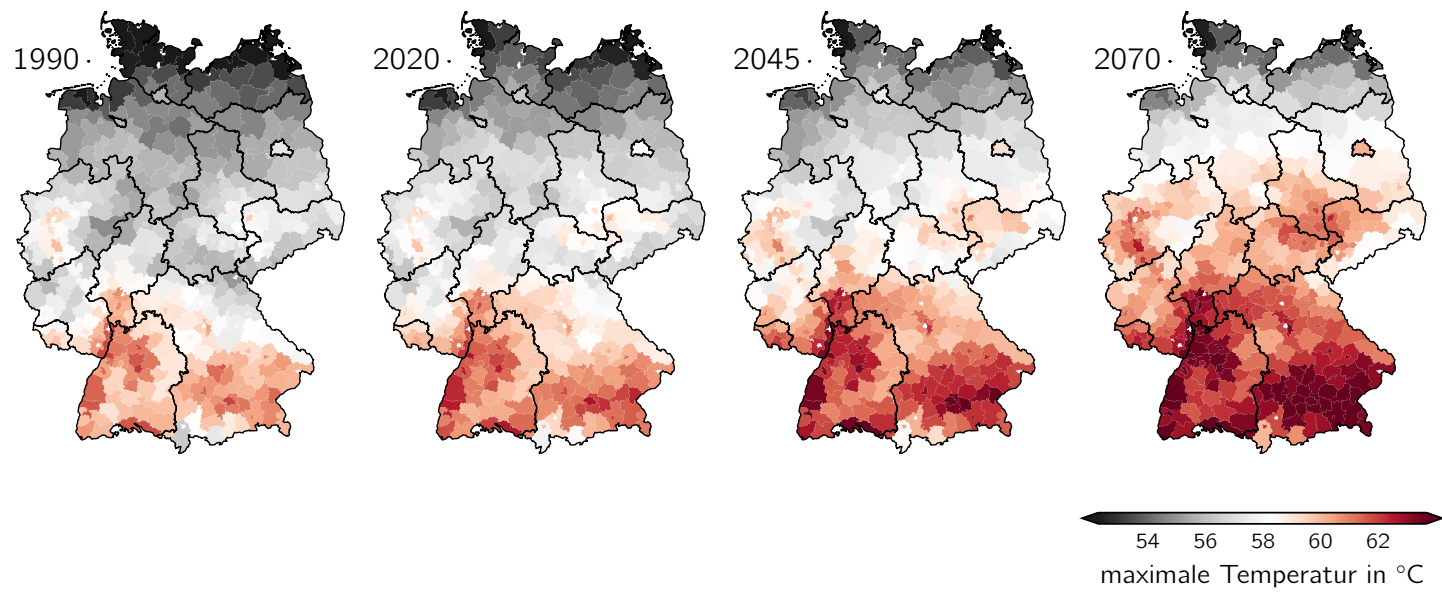

(a) Asphaltdeckschicht
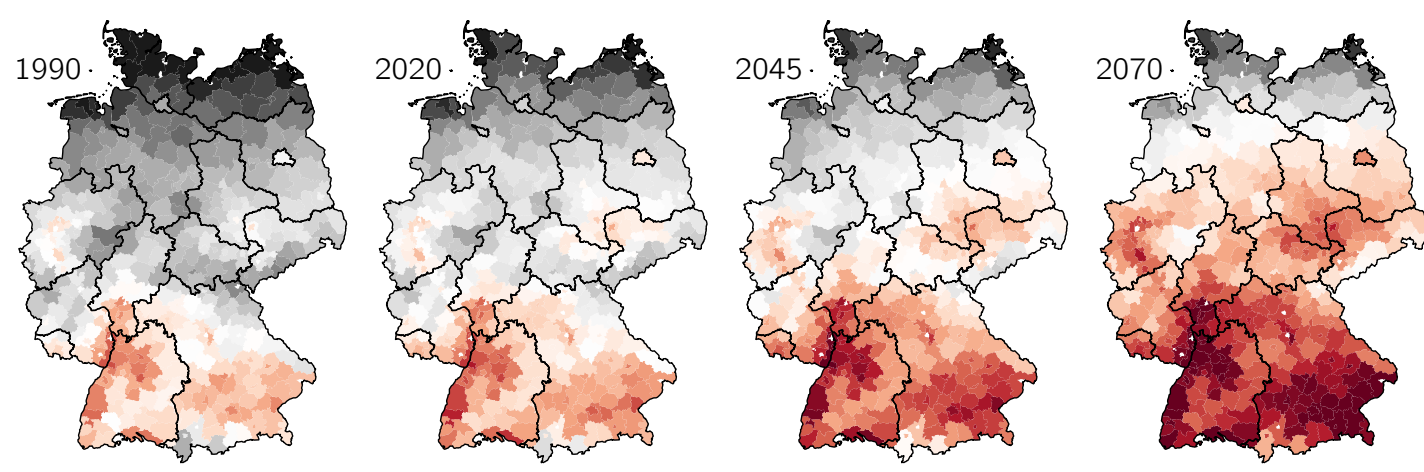

(b) Asphalttragschicht

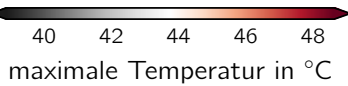

Abbildung 4.5

30-jähriger Maximalwert der Temperatur in Abhängigkeit von der Verkehrsfreigabe

\subsubsection{Anzahl an heißen Tagen in der Asphaltbefestigung}

Die Simulationsergebnisse dieser Arbeit wurden auf die Anzahl der Tage mit einer Temperatur größer gleich $30^{\circ} \mathrm{C}$ für die Asphaltschichten gesondert geprüft. Der Parameter wird nachfolgend als heiße Tage in der Asphaltbefestigung verwendet.

In der Abbildung 4.6 ist die Änderung der Anzahl an heißen Tagen in der Asphaltbefestigung bezogen auf den Wert von 2020 dargestellt. Der Verlauf der Änderung innerhalb der Asphaltdeckschicht zeigt, dass in der Vergangenheit eine recht konstante Anzahl an heißen Tagen vorherrschte. Anhand der Simulationen des thermischen Verhaltens konnten auch keine großen Schwankungen innerhalb der einzelnen Regionen festgestellt werden. Die etwas 


\section{Simulation der thermischen Beanspruchungen}

höheren Temperaturen in den 1970er Jahren führten auch zu geringfügig höheren Temperaturen innerhalb der Asphaltdeckschicht. Die thermischen Bedingungen unter dem Aspekt der heißen Tage konnte als konstant angenommen werden. Die simulierten Kurvenverläufe weisen zukünftig aber eine deutlich höhere thermische Belastung für Straßenkonstruktionen aus. Die Änderung steigt ab dem Jahr 2030 kontinuierlich auf eine mittlere Abweichung von $127 \%$ im Jahr 2070 an.

Betrachtet man die Änderungen innerhalb der Asphalttragschicht, so konnte ein analoge Entwicklung anhand der Simulationen nachgewiesen werden. Die Werte der Vergangenheit weisen geringe zeitliche Schwankungen bei gleichzeitig geringen räumlichen Änderungen auf. Die zukünftigen Werte steigen jedoch deutlich stärker an. Ab dem Jahr 2070 muss mit einer mittleren Zunahme an heißen Tagen in der Asphaltbefestigung auf ca. 160\% gerechnet werden. Somit steigt die Dauer der thermischen Exposition durch sehr hohe Temperaturen innerhalb des Nutzungszeitraums von Straßenkonstruktionen.

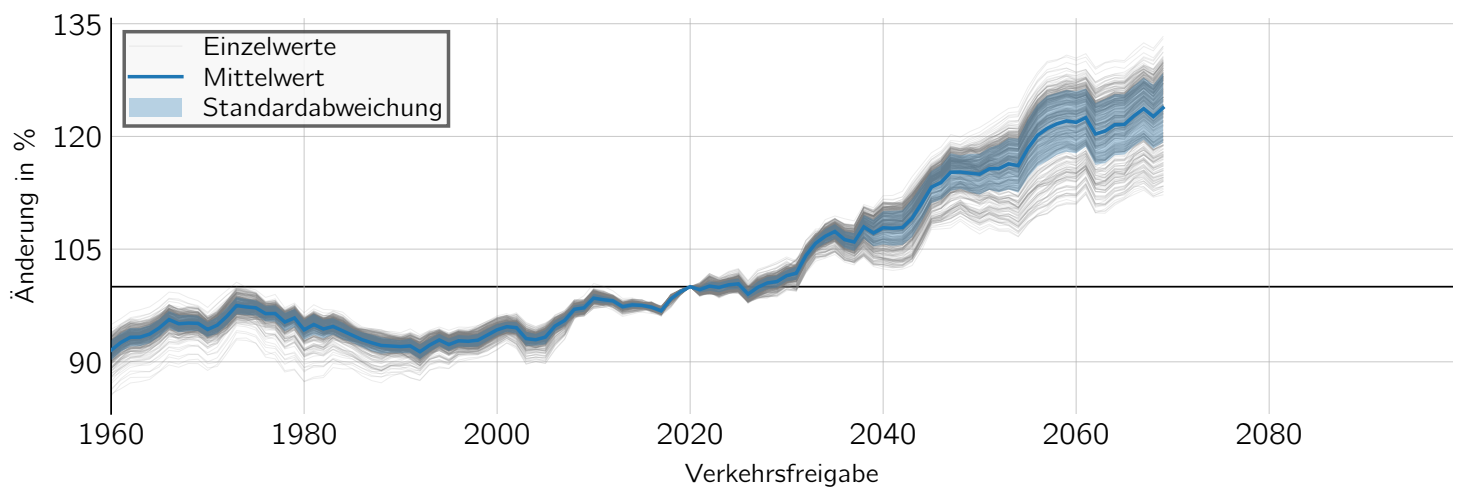

(a) Asphaltdeckschicht

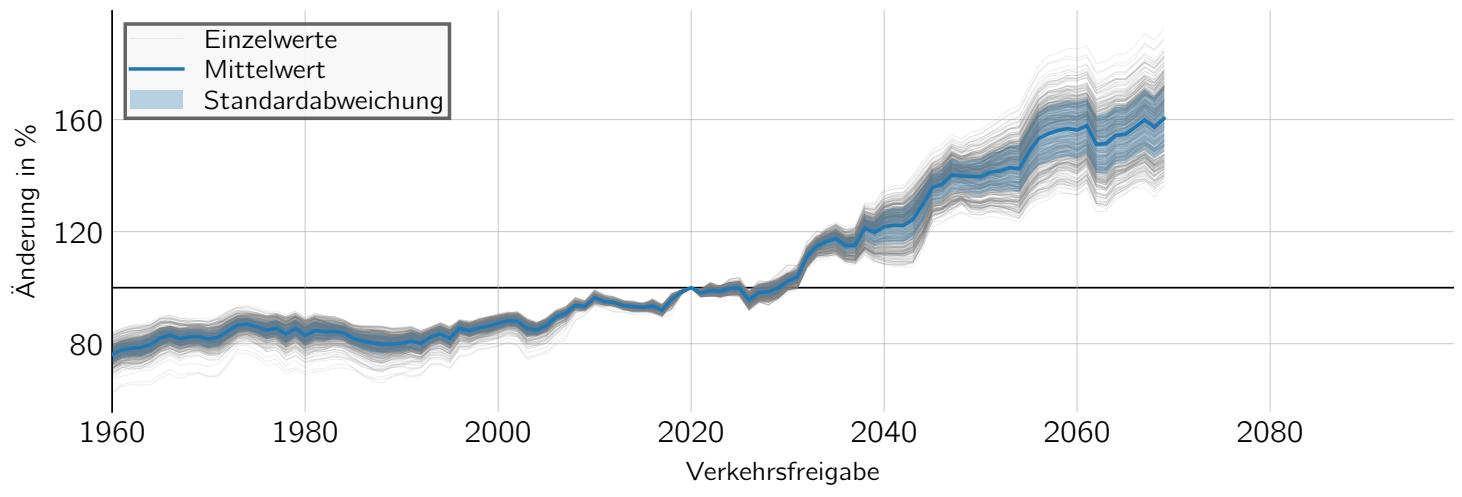

(b) Asphalttragschicht

Abbildung 4.6

Abweichung der heißen Tage in der Asphaltbefestigung bezogen auf den Wert mit der Verkehrsfreigabe von 2020 


\subsubsection{Mittlere Temperaturen}

Die über die Asphaltschicht gemittelte Temperatur bildet einen weiteren Indikator für das Schädigungspotenzial der Straßenkonstruktionen. Eine Verringerung der gemittelten Temperatur hat wahrscheinlich einen positiven Effekt auf die Dauerhaftigkeit der Straßenkonstruktionen. Ein Anstieg der mittleren Temperatur geht mit einer Erhöhung der thermischen Beanspruchung innerhalb der Straßenkonstruktionen einher. Dieser Effekt ist als negativ zu bewerten, da höhere Temperaturen zu einer Absenkung des Steifigkeitsmoduls des Asphaltpaketes führen. Dies verursacht, bei sonst gleichen Randbedingungen, erhöhte Schädigungen durch größere Dehnungen innerhalb der Straßenkonstruktion.

In Abbildung 4.7 sind die räumlichen und zeitlichen Veränderungen der mittleren Temperaturen innerhalb einer 30-jährigen Periode dargestellt. Die Ergebnisse der Simulationen zeigen, dass die Temperaturbedingungen innerhalb der Asphaltdeckschicht und Asphalttragschicht im Jahr 1960 und 2020 nahezu identisch sind. Die über die Fläche gemittelte Temperatur liegt für die Asphaltdeckschicht als auch für die Asphalttragschicht bei ca. $12,90^{\circ} \mathrm{C}$. Dieses Verhalten ändert sich jedoch in Zukunft. Die mittleren Temperaturen im Jahr 2070 liegen mit einem Mittelwert von $16,00{ }^{\circ} \mathrm{C}$ deutlich über den aktuellen Werten.

Stellt man die Änderungen in Abhängigkeit der Zeit dar (Abbildung 4.8), so ist ersichtlich, dass in der Vergangenheit von nahzu konstanten Bedingungen ausgegangen werden konnte. Die mittleren Temperaturen in den einzelnen Regionen unterscheiden sich, jedoch ergaben sich kaum zeitliche Veränderungen. Die Simulationen ergeben, dass ab dem 21. Jahrhundert von einem kontinuierlichen Anstieg ausgegangen werden muss. Die einzelnen Simulationsergebnisse weisen ab ca. 2030 deutliche Differenzen auf, welche sich bis Ende des Betrachtungszeitraums weiter vergrößern. 

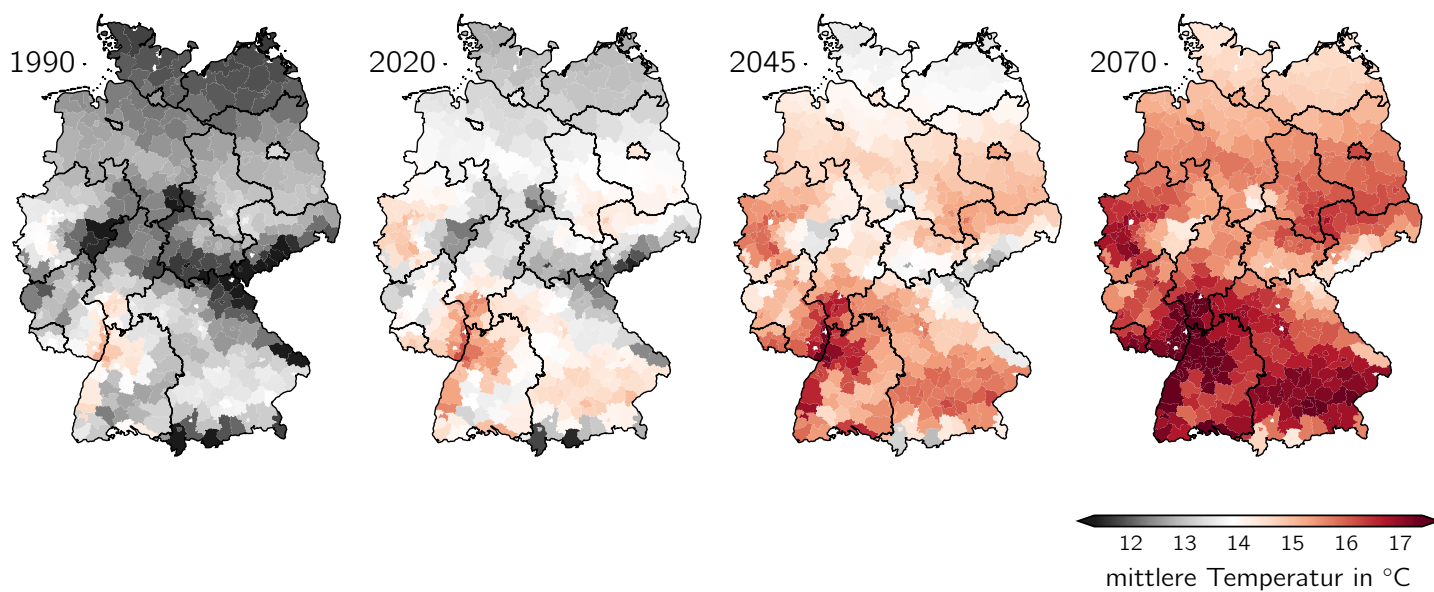

(a) Asphaltdeckschicht
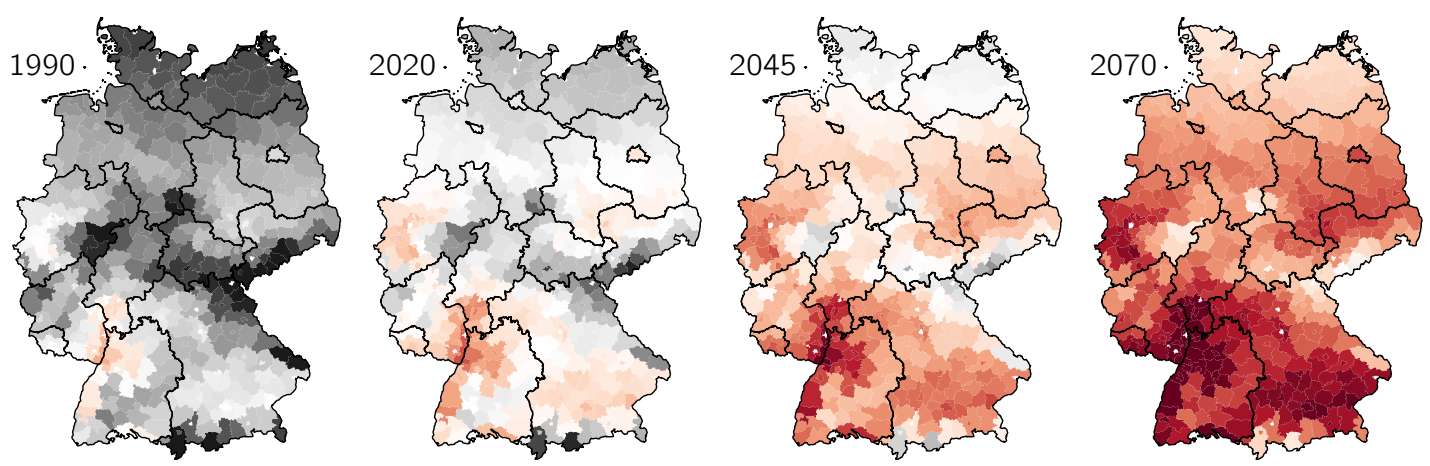

(b) Asphalttragschicht

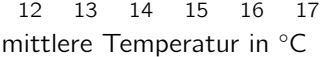

Abbildung 4.7

30-jähriger Mittelwert der Temperatur 


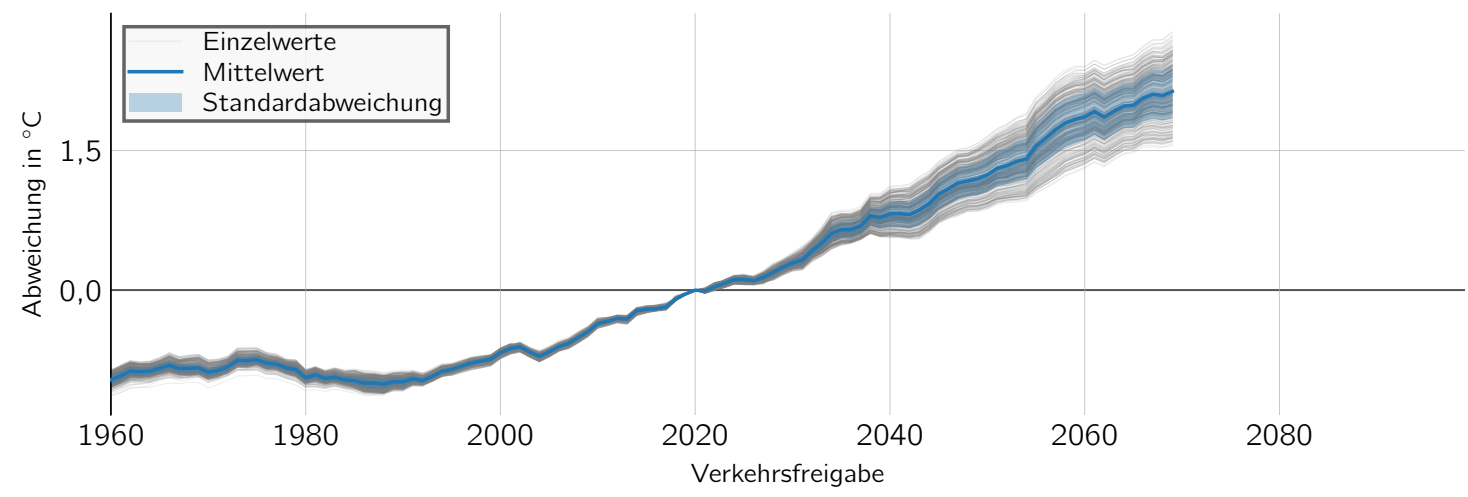

Abbildung 4.8

Abweichung des Mittelwertes der Temperatur innerhalb der Asphaltdeckschicht bezogen auf den Basiswert mit der Verkehrsfreigabe von 2020 


\subsubsection{Anzahl an Tage mit Frost in der Straßenbefestigung}

Aus den Simulationsergebnissen zur Berechnung der Temperaturzustände in den Straßenkonstruktionen können die Anzahl an Tagen mit Frost in allen Asphaltschichten bestimmt werden, wobei die Temperatur in einer Schicht kleiner gleich $0,00^{\circ} \mathrm{C}$ als Frosttemperatur definiert wurde. Weiterhin gilt, dass die Temperatur nicht den gesamten Tag unterhalb der Frosttemperatur liegen muss, sondern schon ein einmaliges Unterschreiten zu einer Wertung als voller Tag führt.

Die Abbildung 4.9 stellt die räumliche Verteilung der Tage mit Frost in der Asphaltbefestigung dar. Die Werte der Vergangenheit zeigen, dass im Bereich der Küstenregionen und entlang der westlichen Grenze eine geringe Anzahl an Tagen mit Frost in der Asphaltbefestigung simuliert wurde. Im Bereich der Mittelgebirge, Alpen und den südlichen Bundesländern ist die Anzahl an Tagen mit Frost signifikant höher. Im Laufe des Betrachtungszeitraums ändert sich Anzahl der Tage jedoch deutlich. Vergleicht man bereits die Verteilung der Tage mit Frost zwischen 1990 und 2020, so ist eine Abnahme der Anzahl sichtbar. Dieser Aspekt gilt sowohl für die Asphaltdeckschicht als auch Asphalttragschicht. In einem besonderen Maße erfolgt die Reduzierung der Tage mit Frost in der Asphaltbefestigung in Thüringen, Sachsen, Bayern und der Alpenregion. Die globale Erwärmung führt dazu, dass sich die Anzahl der Tage mit Frost in der Asphaltbefestigung bis Ende des Betrachtungszeitraums auf weniger als ein Viertel reduziert. Dies gilt gleichbleibend für alle untersuchten Asphaltschichten.

Die Anzahl der Tage mit Frost in der Asphaltbefestigung ist ein Indikator für das Potenzial von Frostschäden, sowohl im Asphaltpaket als auch in den ungebundenen Schichten. Eine Abnahme der Frosttage reduziert die Länge der Exposition und dies hat theoretisch einen positiven Effekt auf die resultierenden Steifigkeitmoduln der Asphalte während der Nutzung. Eine Reduktion der Tage mit Frost in der Asphaltbefestigung könnte jedoch auch eine Erhöhung der Frost-Tau-Prozesse zu Folge haben. Dies könnte in einer gesonderten Analyse bestimmt werden, ist aber nicht Gegenstand dieser Arbeit.

In der Abbildung 4.10 ist die zeitliche Abnahme der Frosttage getrennt für die Asphaltdeckschicht und die Asphalttragschicht dargestellt. Die anfängliche Häufigkeit der Frosttage innerhalb der Asphaltdeckschicht ist deutlich höher als die Anzahl in der Asphalttragschicht. Innerhalb des Betrachtungszeitraums sinkt die Anzahl der Tage kontinuierlich, bis in einigen Regionen Deutschlands ab dem Jahr 2070 keine oder nur sehr wenige Frosttage innerhalb des Nutzungszeitraums auftreten. Die Daten zeigen jedoch nicht, dass der Belastungszustand Frost-Tau-Wechsel in Zukunft nicht mehr auftritt. Die Anforderungen für Straßenkonstruktionen bleiben wie aktuell definiert bestehen. Das konstruktive Maß, also z. B. die Dicke der Frostschutzschicht oder die Anforderungen an die verwendeteten Bitumen, könnte jedoch an die sich ändernden thermischen Beanspruchungen angepasst werden. 

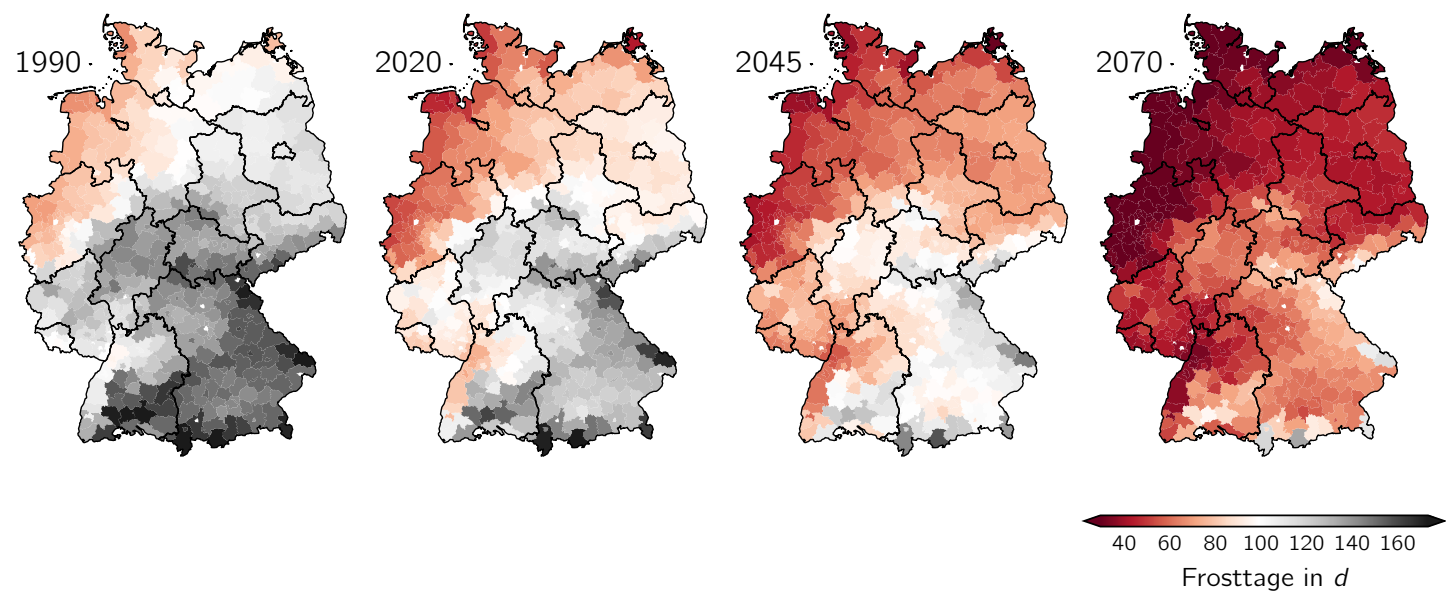

(a) Asphaltdeckschicht
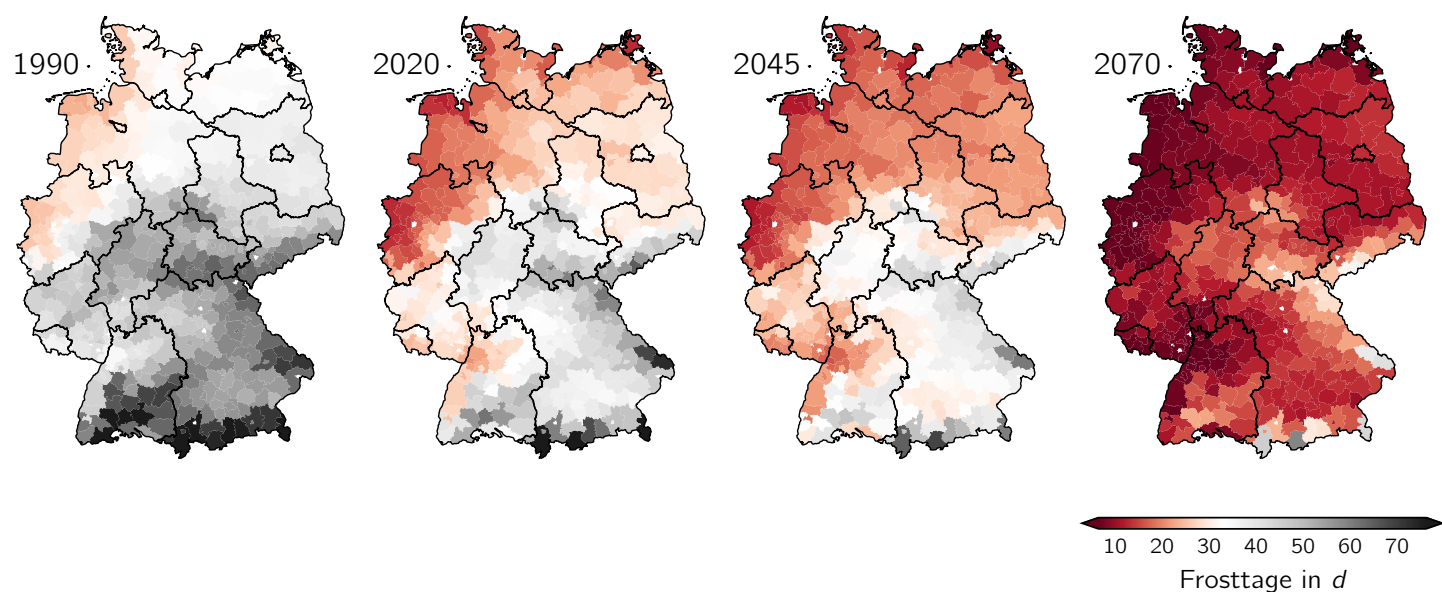

(b) Asphalttragschicht

Abbildung 4.9

Mittlere Anzahl an Tagen mit Frost in der Asphaltbefestigung 


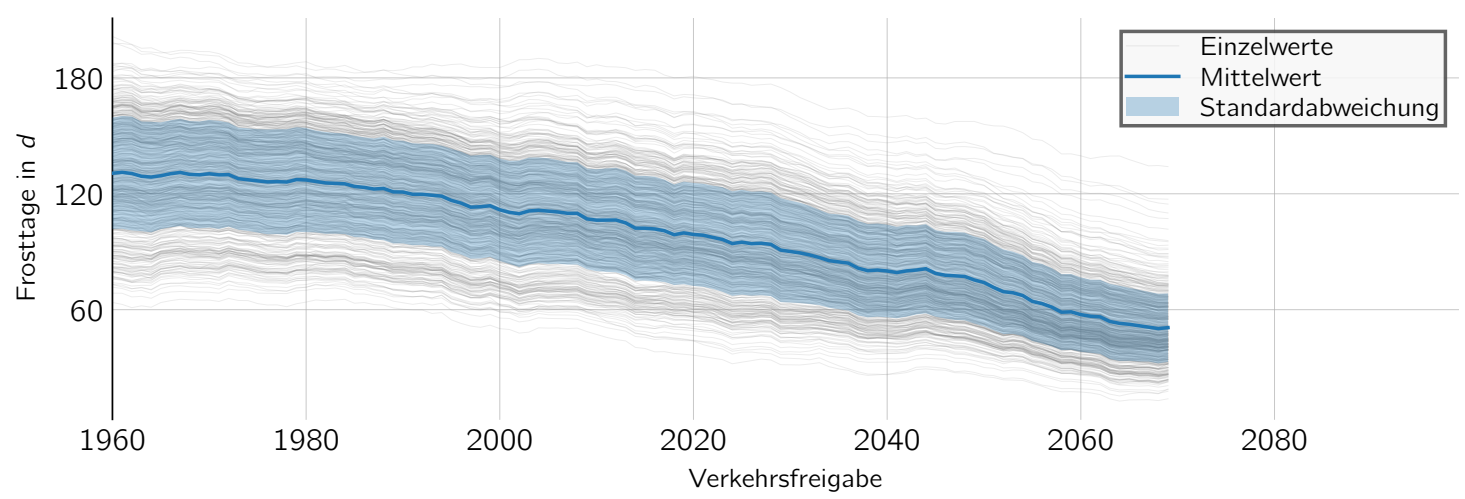

(a) Asphaltdeckschicht

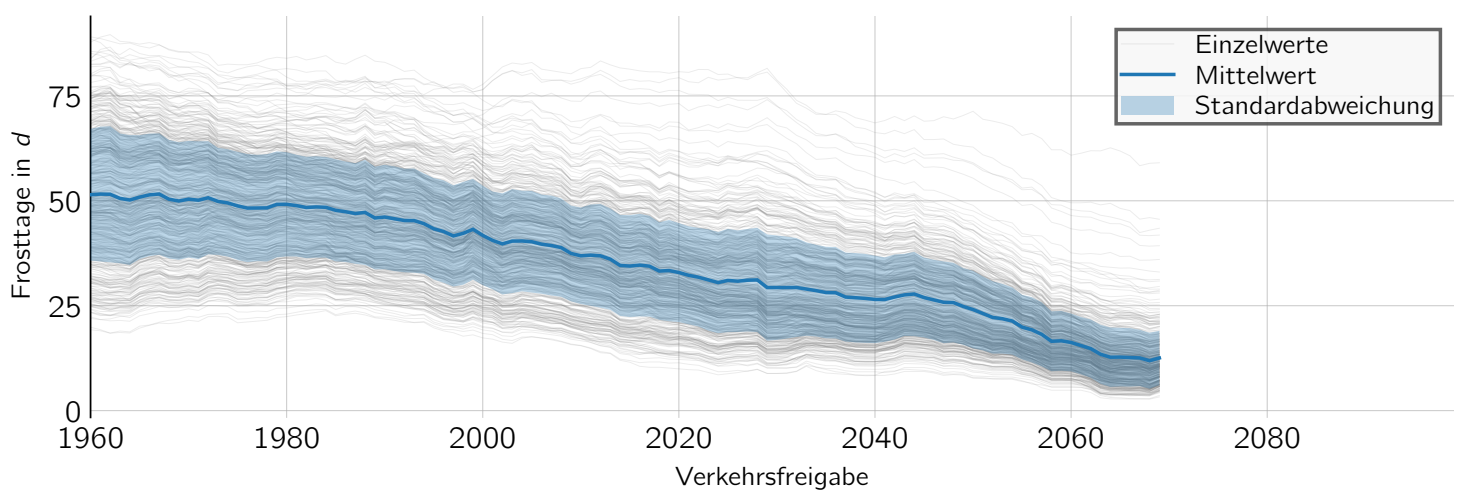

(b) Asphalttragschicht

Abbildung 4.10

Entwicklung der Anzahl an Frosttagen aller untersuchten Regionen 


\subsubsection{Zusammenfassung}

Die klimatischen Bedingungen der untersuchten Regionen bestimmen das thermische Verhalten von Straßenkonstruktionen. Die prognostizierten Veränderungen durch den Klimawandel, vor allem die Erhöhung der mittleren Lufttemperaturen und der Anstieg des eingehenden Strahlungswärmestroms, führen zu einer langfristigen kontinuierlichen Steigerung der StraBentemperaturen (Abbildung 4.11). Im Mittel erhöhen sich die maximalen Temperaturen innerhalb der Asphalttragschicht um ca. $5 \mathrm{~K}$ und die minimalen Temperaturen um bis zu $8 \mathrm{~K}$. Dies entspricht einer deutlich stärkeren Erhöhung als die zukünftig prognostizierte globale Steigerung der mittleren bodennahen Lufttemperatur (vgl. Abschnitt 4.3).

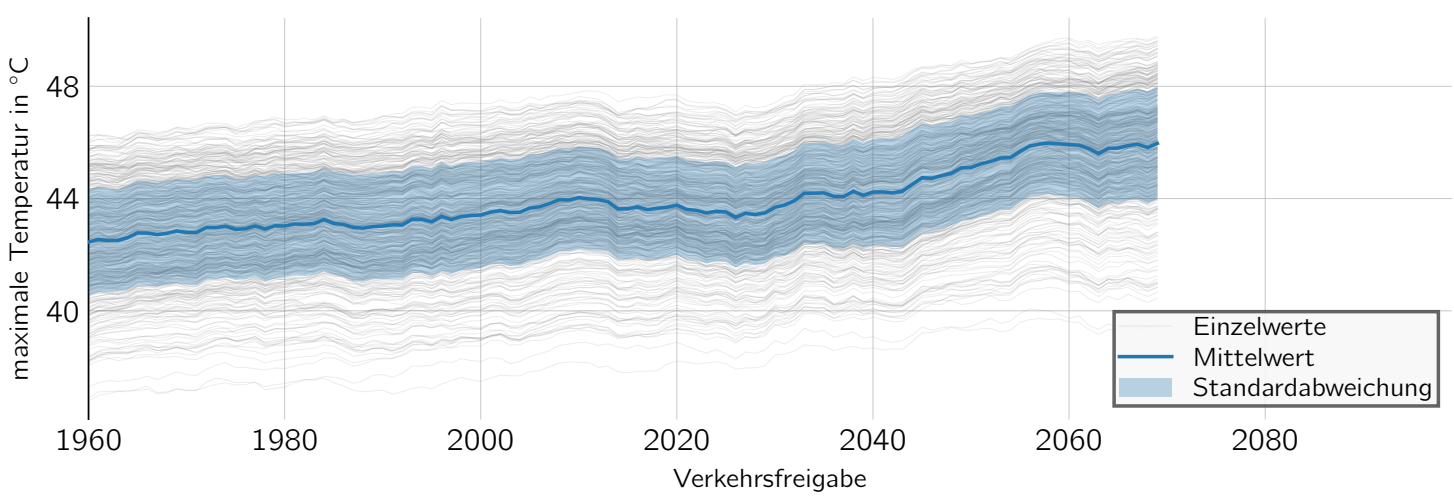

(a) maximale Temperaturen

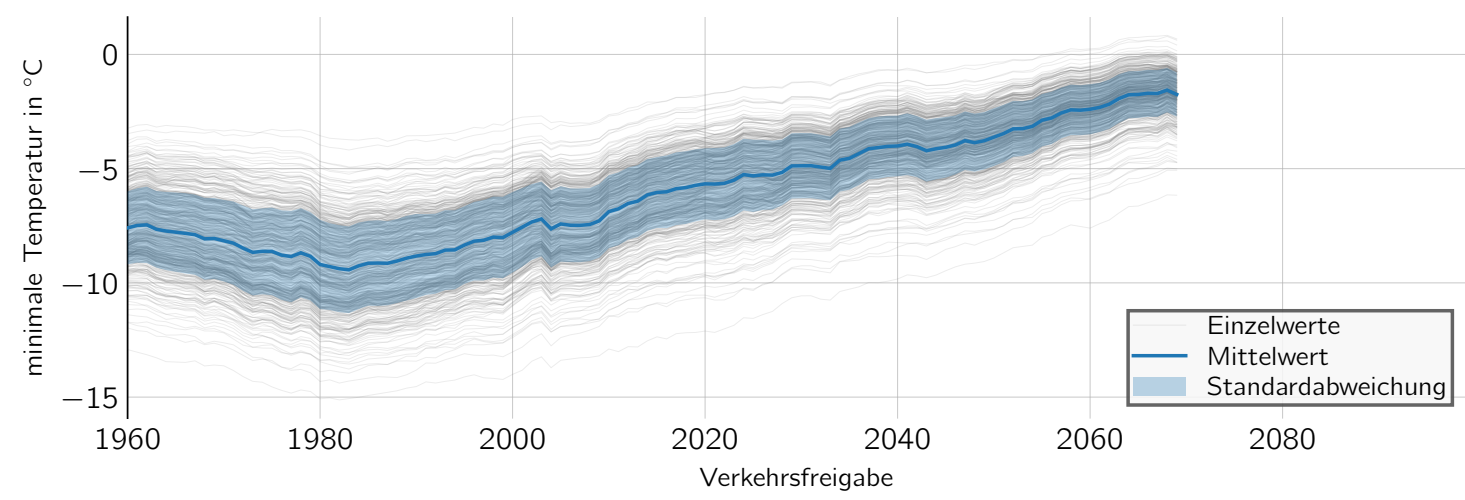

(b) minimale Temperaturen

Abbildung 4.11

Veränderung des Minimalwertes der Temperatur in der Asphalttragschicht aller untersuchten Regionen

Die einzelnen Regionen in Deutschland unterliegen einer deutlichen Variabilität der resultierenden Temperaturen innerhalb der Straßenkonstruktionen. Im gesamten Bundesgebiet steigen die maximalen, mittleren und minimalen Temperaturen unter den festgelegten Randbedingungen kontinuierlich an. Die regionalen Klimabedingungen führen jedoch dazu, dass 


\section{Simulation der thermischen Beanspruchungen}

nicht jede Region im gleichen Maße von den Folgen des Klimawandels betroffen ist. Es muss davon ausgegangen werden, dass die Dauerhaftigkeit von Straßenbefestigungen in Asphaltbauweise regional unterschiedlich stark beeinflusst wird.

Die Bewertung der Ergebnisse mittels einer Changepoint Analyse ${ }^{1}$ (Killick, Fearnhead und Eckley, 2012) ergibt (Abbildung 4.12), dass eine Einteilung der klimatischen Bedingungen ab 1960 in zeitlich unterschiedliche Abschnitte möglich ist. Jeder Abschnitt beschreibt dabei einen Zeitraum, in dem signifikante Änderungen gegenüber dem vorhergehenden Abschnitt auftreten. Unter den Bedingungen der Changepoint Analyse ergeben sich mehrere Abschnitte. Die Periode 1960 bis ca. 2010 wurde in drei von vier Varianten als homogener Abschnitt erkannt. Sowohl die mittleren Temperaturen als auch die Anzahl der heißen Tage und Frosttage in den Asphaltschichten sind nahezu konstant. Die Analyse der maximalen Temperaturen ergibt zwei Zeiträume, wobei die Anstiege der linearen Trendwerte nur geringfügig voneinander abweichen. Die Ergebnisse der Gegenwart sind unter den aufgeführten Bedingungen nahezu identisch, wobei ab den 2030er Jahren mit einer signifikanten Abweichung zu den aktuellen Werten gerechnet werden muss. Die Berechnungen der Temperaturen innerhalb der Straßenkonstruktionen zeigen zum Ende dieser Periode einen deutlichen Sprung bei den auftretenden maximalen Temperaturen an. Innerhalb der Periode kommt es kontinuierlich zu einer Erhöhung der mittleren Temperaturen bei einem gleichzeitigen Absinken der Frosttage in den Asphaltschichten.

In den folgenden Perioden steigen die mittleren Temperaturen innerhalb der Asphalttragschicht kontinuierlich an, wobei sich der Zuwachs nach 2030 und 2050 von den vorhergehenden Abschnitten unterscheidet.

Dies deutet darauf hin, dass Straßenkonstruktionen besonders kritisch auf die sich ändernden klimatischen Bedingungen reagieren.

Daraus kann abgeleitet werden, dass diese Veränderungen zukünftig im Dimensionierungsverfahren berücksichtigt werden müssen - entweder mit einer in der Planungsphase etwas aufwändigeren Berücksichtigung regionaler Gegebenheiten oder mit einer in der Ausführung teureren überregionalen Annahme des worst-case für das gesamte Bundesgebiet.

\footnotetext{
${ }^{1}$ Verfahren nach Belt, minimaler zeitlicher Abstand: 1 Jahr, kernel: radial basis function
} 


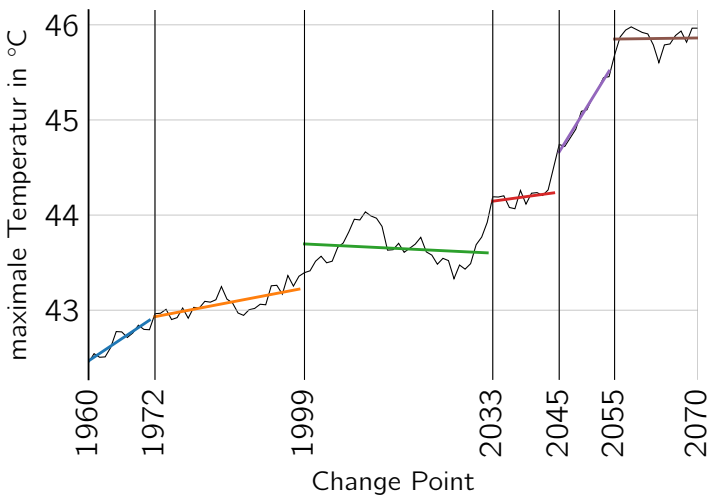

(a) Maximale Temperaturen

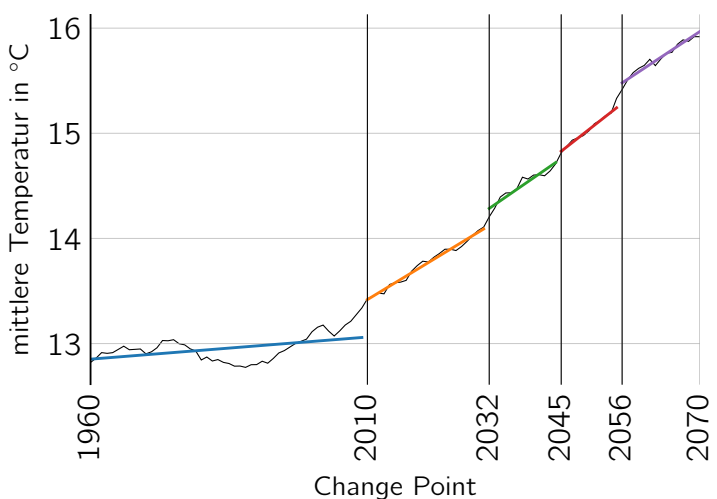

(c) Mittlere Temperaturen

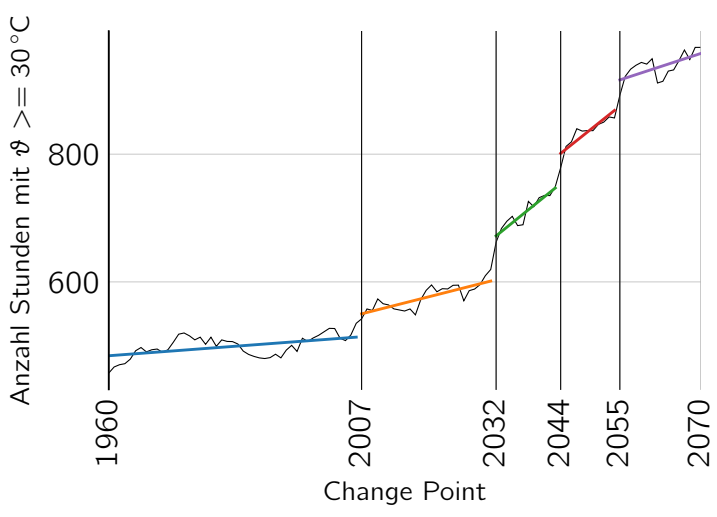

(b) Heiße Tage

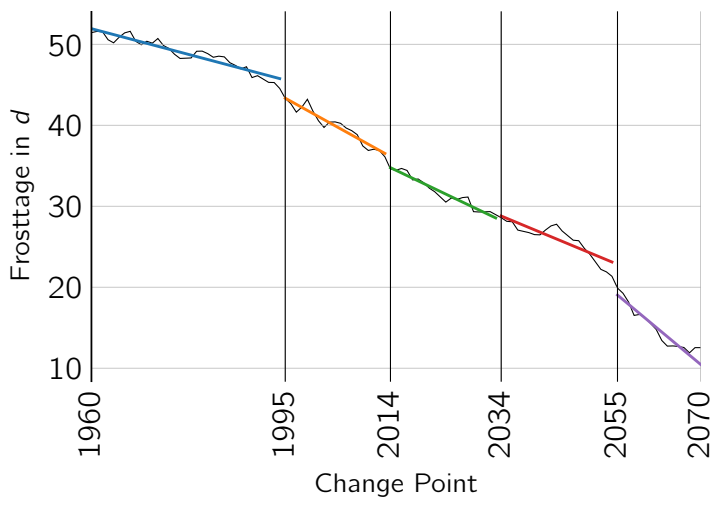

(d) Frosttage

Abbildung 4.12

Changepoint Analyse für unterschiedliche Parameter bezogen auf den Einfluss der thermischen Eigenschaften der Asphalttragschicht 



\section{Clusteranalyse}

In vielen Bereichen wie der Medizin, Wirtschaft oder Wissenschaft werden Daten erfasst und Zusammenhänge zwischen Merkmalen der Daten gesucht. Ziel dabei ist es, Zusammenhänge oder Ähnlichkeiten zwischen Datensätzen zu identifizieren und somit die Struktur von Daten zu bewerten. Der primäre Aspekt der Clusteranalyse ist, anhand von verfügbaren Daten die Beziehung der Einzelelemente zueinander zu beschreiben und homogene Subgruppen innerhalb der Datenstruktur zu definieren. Gleichzeitig ist es sinnvoll, dass die untersuchte Struktur eine große Heterogenität besitzt, also die Gruppen möglichst unähnlich zueinander sind. Dies erfolgt durch eine schrittweise Agglomeration der einzelnen Datensätze zu Clusterzentren. Diese Zentren beschreiben wiederum die mittleren Eigenschaften der zugehörigen Daten (Lletí u. a., 2004; Backhaus u. a., 2016).

Zur Bildung dieser Cluster stehen differenzierte mathematische Modelle zur Verfügung. Grundsätzlich kann zwischen hierarchischen und partinionierenden Modellen unterschieden werden. Die einzelnen Verfahren unterscheiden sich in zwei Aspekten. Erstens gibt es verschiedene Methoden um das Distanzmaß (Proxitätsmaß) zwischen den Objekten zu berechnen. Zweitens unterscheiden sich die Vorgehensweisen nach denen ähnliche Objekte zusammengefasst werden (Gruppierungsverfahren).

In dieser Arbeit soll die Clusteranalye verwendet werden, um aus den simulierten Temperaturbedingungen in der Straßenkonstruktion (siehe Kapitel 4) eine endliche Menge repräsentativer Klassen von Temperaturprofilen zu erstellen. Diese Temperaturprofile können dann als Eingangsdaten für die Dimensionierungsrechnungen verwendet werden. Dazu wurden mehrere Verfahren wie k-Means, k-Median, Ward hierarchical clustering oder DBSCAN verwendet und deren Eignung geprüft. Die Ergebnisse wurden untereinander verglichen und bewertet. Der k-Means Algorithmus wurde als Vorzugsvariante ausgewählt, da die Berechnungsgeschwindigkeit bei der Anzahl an Daten sehr schnell ist, bei einer gleichzeitig hohen Güte der Ergebnisse. Bedingt durch die Komplexität der Algorithmen wurde auf die Beschreibung der nicht verwendeten Verfahren verzichtet.

Nachfolgend werden das k-Means Verfahren und dessen Funktionsweise im Detail beschrieben. Weiterhin werden die Ergebnisse der Clusteranalysen von Temperaturbedingungen in Straßenkonstruktionen mit den Verfahren nach den RDO Asphalt (2009) sowie zu den normierten charakteristischen Temperaturprofilen (ncTs) nach Kayser (2007) verglichen. 


\section{Clusteranalyse}

\section{1 k-Means Algorithmus}

Der k-means Algorithmus ist ein Clusterverfahren der Kategorie partitionierende Verfahren. Dabei müssen Anwender die Anzahl an Clustern festlegen und mittels des Algorthmus werden die Clusterzentren in Abhängigkeit der Merkmale bestimmt. Die Eigenschaften der Clusterzentren leiten sich aus den Mittelwerten der zugehörigen Einzeldaten ab.

Mittels des k-Means Algorithmus können nicht klassifizierte und mehrdimensionale Daten analysiert und in Abhängigkeit der Merkmale (features) in Cluster mit ähnlichen Merkmalen segmentiert werden. Dabei wird ein einfaches Konzept zur Bestimmung der optimalen Zugehörigkeit der Einzelwerte zu den Clustern genutzt. Grundlegend sind zwei Arbeitsschritte bei der Analyse notwendig:

1. Die Mittelwerte aller Merkmale der Einzelpunkte eines Clusters bilden ein Clusterzentrum.

2. Jeder Einzelwert liegt näher am eigenen Clusterzentrum als zu einem anderen Clusterzentrum.

Bedingt durch die Komplexität der Anwendung, nämlich die Clusteranalyse von zeitabhängigen Temperaturprofilen in Straßenkonstruktion, wurde das recht einfache jedoch schnelle Verfahren k-means für die Bestimmung der Cluster gewählt. Die erhöhte Berechnungsgeschwindigkeit im Vergleich zu anderen Verfahren erlaubt eine mehrfache Wiederholung der Analyse zur Steigerung der Güte der Ergebnisse. Ein erheblicher Nachteil des Verfahrens ist jedoch, dass die Anzahl an Clustern bereits vor der Durchführung der Analyse bekannt sein muss. Da im Regelfall die Anzahl der Cluster unbekannt ist, muss dies mittels eines geeigneten Verfahrens für jeden Fall neu bestimmt werden.

Nachfolgend wird der Prozess der Clusterbildung an einem vereinfachten Beispiel dargestellt. Dabei wurde ein Testdatensatz aus Zufallszahlen um vier Clusterzentren mit einer definierten Standardabweichung der Einzelparameter generiert. Jeder Datenpunkt umfasst dabei zwei Merkmale (Features), welche mit den Parametern a und b gekennzeichnet sind (Abbildung 5.1).

Für dieses Beispiel wird angenommen, dass die Anzahl der Cluster bekannt ist und die Analyse mit einer Clusteranzahl (k) von vier Clustern ausgeführt wird. Als erster Arbeitsschritt erfolgt eine zufällige Festlegung der vier Clusterzentren (5.2). Für diesen Schritt sind mehrere Methoden vorhanden. Unter anderem kann die Initialisierung der Clusterzentren anhand $\mathrm{k}$ zufälliger Elemente der Grundgesamtheit gewählt werden oder es erfolgt eine Generierung von Zufallsparametern innerhalb der Grenzen der einzelnen Merkmale. In diesem Beispiel wurde die zweite Variante verwendet. Die Clusterzentren sind in der Abbildung farblich markiert. 


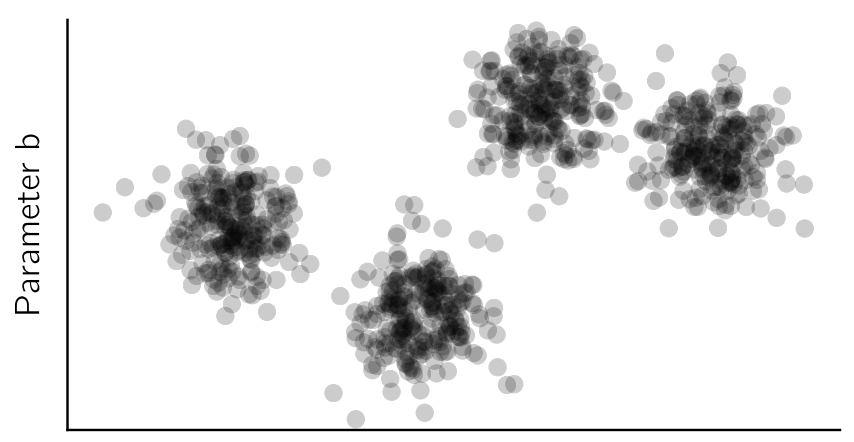

Parameter a

Abbildung 5.1

Beispieldaten mit vier Clusterzentren und zwei Merkmalen

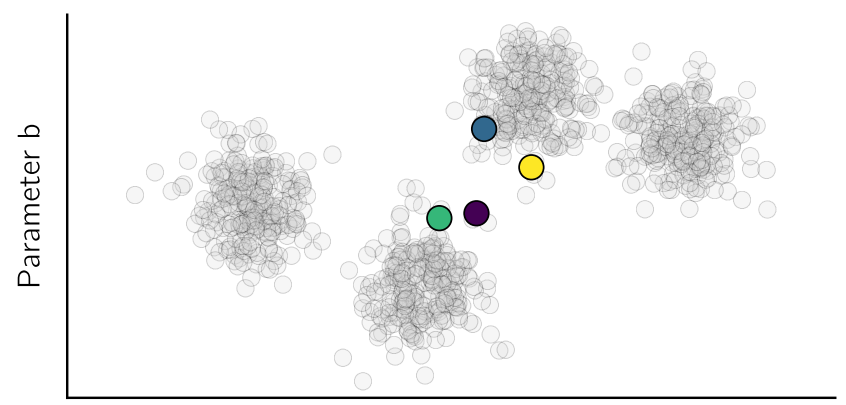

Parameter a

Abbildung 5.2

Initiale Festlegung der Clusterzentren

Mittels der gewählten Clusterzentren wird zwischen diesem und jedem Einzelwert die Euklidische Entfernung nach

$$
d=\sqrt{\sum_{i=1}^{n}\left(q_{i}-p_{i}\right)^{2}}
$$

berechnet. Für jeden Einzelwert werden die Entfernungen zu allen Clusterzentren bestimmt und der Klasse mit der geringsten Entfernung zugeordnet (Abbildung 5.3a, Schritt 1). Anschließend werden die Parameter der Clusterzentren anhand aller zugeordneten Einzelwerte neu berechnet (Abbildung 5.3b, Schritt 1). Dies erfolgt durch die Mittelwertbildung jedes einzelnen Merkmals der zugeordneten Elemente. Der Gesamtfehler innerhalb der Analyse wird durch eine Fehlerbetrachtung, meist die Summe der kleinsten Quadratfehler bestimmt (Carvalho u. a., 2016). 


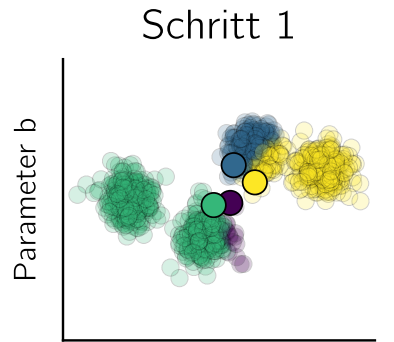

Parameter a

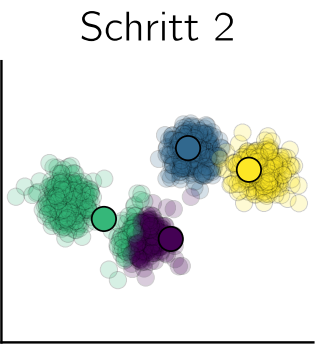

Parameter a
Schritt 3

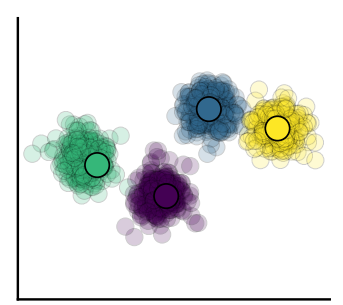

Parameter a
Schritt 4

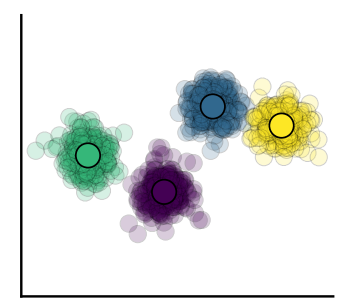

Parameter a

(a) Berechnung der Entfernung aller Einzelpunkte zu den Clusterzentren

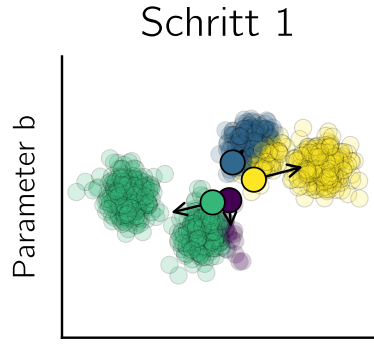

Parameter a
Schritt 2

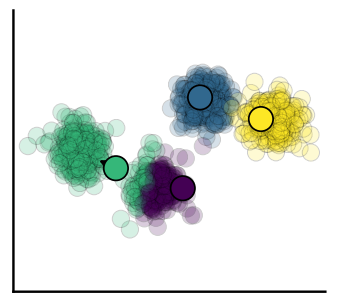

Parameter a
Schritt 3

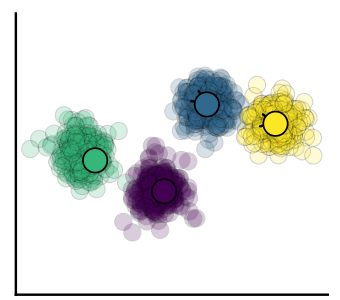

Parameter a
Schritt 4

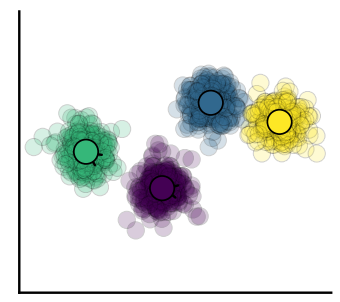

Parameter a

(b) Zuordnung Einzelwerte zu den Clusterzentren

Abbildung 5.3

Veränderung der Zuordnung der Einzelwerte zu den Clusterzentren in Abhängigkeit der Iterationsschritte des k-Means Algorithmus

Im zweiten Schritt erfolgt die erneute Bestimmung des Distanzmaßes zwischen den Einzelpunkten und den Clusterzentren. Auf Basis dieser Zuordnung wird eine Neuberechnung der Merkmale der Clusterzentren anhand der mittleren Eigenschaften durchgeführt. Die Arbeitsschritte werden iterativ solange wiederholt, bis eine definierte Abweichung zwischen den im Schritt $i$ und $i-1$ berechneten Merkmalen der Clusterzentren unterschritten wird.

In Abbildung 5.4 wurde die berechnete Abweichung (mittlere euklidische Distanz) zwischen den Einzelwerten und den Clusterzentren in Abhängigkeit der Iterationsschritte dargestellt. Im ersten Schritt wurde eine sehr hohe Abweichung berechnet. Nach Neuberechnung der Clusterzentren im Schritt 2 konnte eine Verminderung in allen Clustern festgestellt werden. Die Durchführung weiterer Iterationen führt in diesem Beispiel zu einer kontinuierlichen Reduktion der Abweichungen zwischen den Merkmalen der zugehörigen Elemente und der mittleren Eigenschaften der Clusterzentren, bis die Iteration am definierten Konvergenzkriterium abgebrochen wird.

Die Zuordnung der Daten zu den Clusterzentren hängt von den gewählten Startparametern der Clusterzentren ab. Um den numerisch bedingten Fehler bei der Analyse zu minimieren, sollten Clusteranalysen mehrfach wiederholt werden. Dabei werden die Merkmale der 


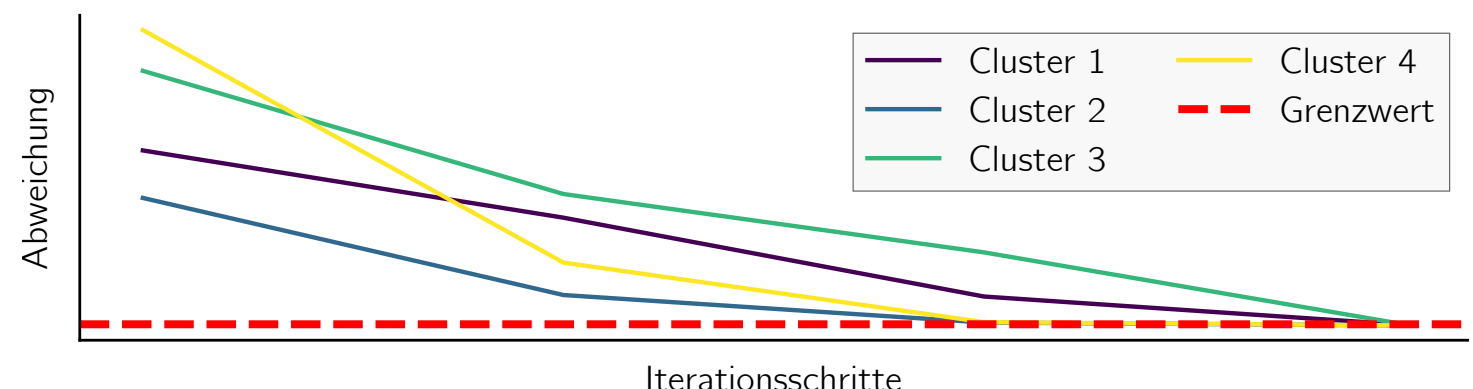

Abbildung 5.4

Änderung der Abweichungen zwischen den Einzelwerten und den Clusterzentren in Abhängigkeit der Iterationsschritte

Clusterzentren zu Beginn der Analysen in Abhängigkeit des Verfahrens mehrfach neu gewählt und jeweils die Arbeitsschritte bis zum Erreichen des Grenzwertes der Abweichung wiederholt. Abschließend erfolgt eine Mittelung der einzelnen Merkmale aller durchgeführten Einzelbetrachtungen. Bei den nachfolgend dargestellten Beispielen in dieser Arbeit wurde das Verfahren mindestens 1000-mal wiederholt, um eine hohe Güte der Analyse sicherzustellen.

Da im Normalfall die Anzahl der Cluster (k) vor der Analyse nicht bekannt ist, ergibt sich das Problem, dass sich die Zuordnung der Einzelpunkte mit der Wahl von $k$ ändert. Bei einer zu geringen Anzahl an gewählten Clustern (zu kleines k) ist die Segmentierung der Daten nicht ausreichend genau. Ist $k$ zu groß gewählt worden, ergeben sich möglicherweise Cluster, die entweder nicht eindeutig getrennt sind oder welche sich kaum voneinander unterscheiden. Beispielhaft ist dieser Sachverhalt für das oben definierte Beispiel dargestellt. Wählt man die Anzahl an Clustern der Analyse zu klein $(k=2)$, ergibt sich keine gute Trennung zwischen den Clusterzentren (Abbildung 5.5). Dahingegen führt ein zu großer Wert $(k=7$ oder $k=10)$ zu einer Fragmentierung der einzelnen Cluster. Die mittleren Eigenschaften der Clusterzentren würden sich in diesem Falle verändern. Weiterhin entstehen unnötig viele Unterteilungen, welche bei den nachfolgenden Dimensionierungsrechnungen zu einer erhöhten Berechnungszeit führen. Für die Wahl einer optimalen Anzahl stehen Verfahren wie die Elbow Method (Yan, 2005), Gap Statistics (Pham, Dimov und Nguyen, 2005; Carvalho u. a., 2016) oder Silhouette Method (Rousseeuw, 1987; Bador u. a., 2015) zur Verfügung. 


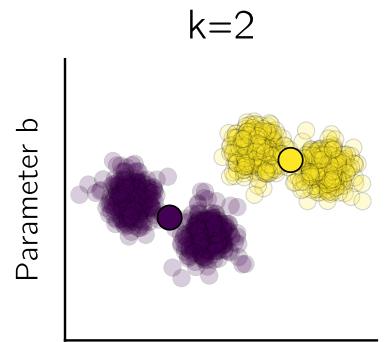

Parameter a

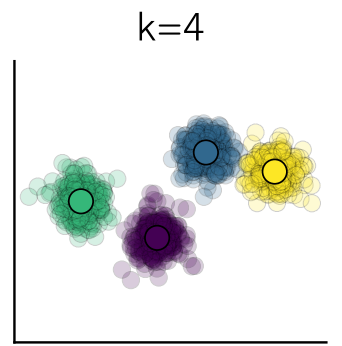

Parameter a

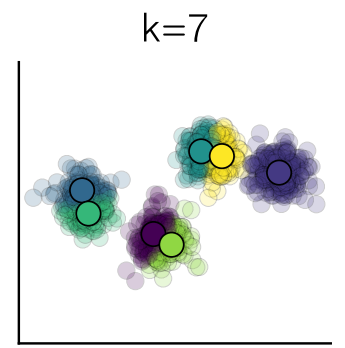

Parameter a

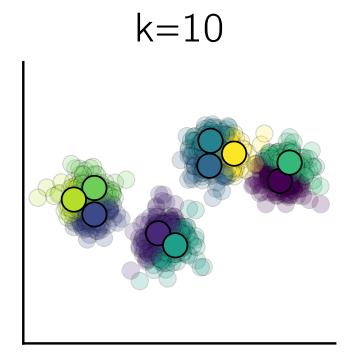

Parameter a

Abbildung 5.5

Ergebnisdarstellung der Clusteranalyse mit variabler Anzahl an definierten Clustern

\subsection{Vergleich der Verfahren}

Die Temperaturprofile aus den Simulationen der thermischen Beanspruchungen können mittels des oben beschriebenen Clusterverfahrens analysiert werden. Die resultierenden Temperaturprofile der Clusterzentren werden nachfolgend als Mittelwertfunktionen bezeichnet. Die einzelnen Verläufe der Mittelwertfunktionen besitzen also die gemittelten Eigenschaften (Temperaturbedingungen) aller zugeordneten Einzelprofile. Zum Vergleich wurden die Simulationen der thermischen Beanspruchungen nach dem Verfahren der RDO Asphalt (2009) und nach Kayser (2007) analysiert. Beide Verfahren beruhen dabei auf der Klassifizierung der einzelnen Zustände anhand der Oberflächentemperatur. Im Verfahren zur Bestimmung der ncTs nach Kayser (2007) werden zusätzlich die täglichen Schwankungen der Temperaturen auf der Fahrbahnoberfläche berücksichtigt. Beide Verfahren finden unter anderem Anwendung im wissenschaftlichen Bereich.

Exemplarisch werden nachfolgend die Ergebnisse der Clusteranalyse und die ermittelten Häufigkeitsverteilungen für das Stadtgebiet Dresden betrachtet. Die Verläufe der Mittelwertfunktionen und ncTs sind in Abbildung 5.6 für den Zeitraum von 2020 bis 2049 dargestellt. Im Hintergrund der Abbildungen sind die simulierten Temperaturen innerhalb der Straßenkonstruktion dargestellt. Die Häufigkeiten des Auftretens der Temperaturzustände sind durch die Sättigung der Farbe visuell gekennzeichnet.

Die als Linien dargestellten repräsentativen Temperaturprofile der Mittelwertfunktionen verlaufen innerhalb des simulierten Temperaturbereiches und die Mehrheit der Clusterzentren liegen im mittleren Temperaturspektrum. Die Dicke der einzelnen Verläufe gibt jeweils die Häufigkeit des Auftretens innerhalb der Periode an. Je dicker die Strichstärke ist, desto häufiger wurden diese Zustände ermittelt. Die Schwerpunkte der Häufigkeitsverteilungen der Mittelwertfunktionen und der Ausgangsdaten liegen übereinander. Die standardisierten Verläufe der ncTs sind in Abbildung 5.6 (b) dargestellt. In den Randbereichen verlaufen die vorab nach Auftreten gefilterten ncTs nicht innerhalb des simulierten Temperaturspektrums. Es werden vor allem im Bereich der Asphalttragschicht unrealistische Verläufe ermittelt. Die Strichstärke der Einzelverläufe geben hier ebenfalls die Häufigkeit des Auftretens an und 


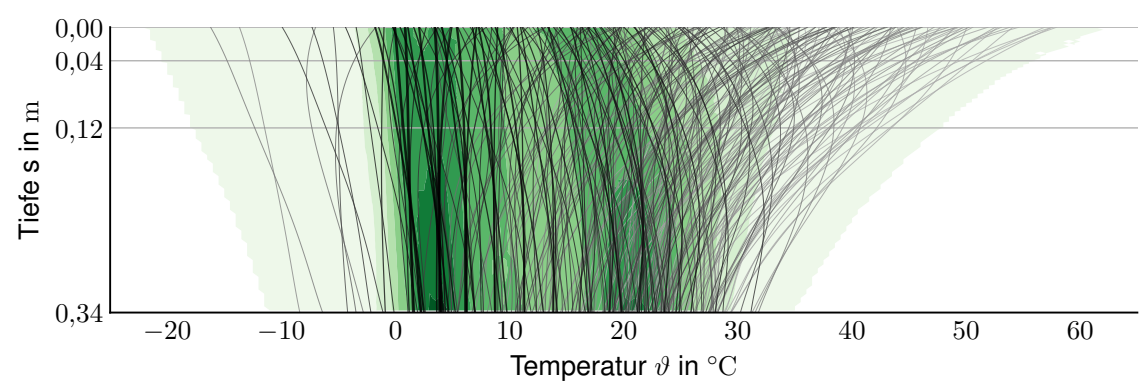

(a) Mittelwertfunktionen

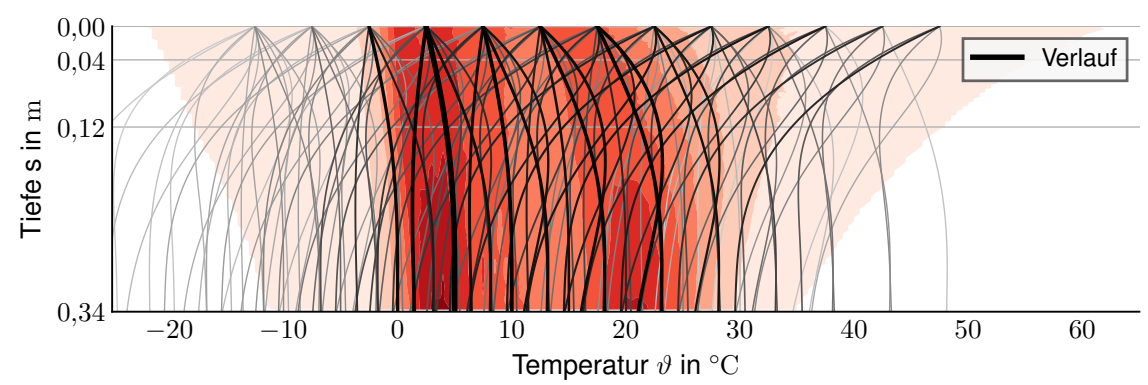

(b) normierte charakteristische Temperaturprofile

Abbildung 5.6

Relevante Temperaturprofile nach den Analysen, Hintergrund entspricht dem ermittelten Temperaturspektrum und Farbe kennzeichnet die Häufigkeit des Auftretens, Betrachtungszeitraum 2020 - 2049, Stadtgebiet Dresden

diese stimmen nicht mit dem Kernbereich der simulierten Temperaturzustände überein. Vor allem die starken Abweichungen im hohen Temperaturbereich sind als kritisch zu bewerten, da unter diesen Temperaturzuständen verstärkte Schädigungen der Straßenkonstruktion auftreten.

Betrachtet man im Detail die Abweichung zwischen den Clusterzentren und den einzelnen Temperaturprofilen so zeigt sich, dass zwischen den Verfahren nach RDO Asphalt (2009), den ncTs sowie den Mittelwertfunktionen sehr große Unterschiede bestehen. In Abbildung 5.7 sind jeweils eine Klasse der unterschiedlichen Verfahren mit einer Oberflächentemperatur von ca. $2,50{ }^{\circ} \mathrm{C}$ vergleichend dargestellt. Die Diagramme zeigen, dass die Verfahren nach den RDO Asphalt (2009) sowie nach den ncTs erhebliche Abweichungen zu den simulierten Temperaturprofile aufweisen. Besonders im Bereich der Asphalttragschicht ergeben sich Differenzen zum Referenzwert von deutlich mehr als $10 \mathrm{~K}$. Der Algorithmus zur Bestimmung der Mittelwertfunktionen ergibt eine signifikante Reduktion der Abweichungen innerhalb der gesamten Tiefe der Straßenkonstruktion. Es konnte eine Reduktion der Abweichungen auf weniger als $5 \mathrm{~K}$ nachgewiesen werden. Weitere Beispiele sind dem Anhang B beigefügt. Vergleicht man weiterhin den Verlauf der Temperaturprofile in der Tiefe, so ist ersichtlich, dass die Variante nach den RDO Asphalt (2009) und nach Kayser (2007) den tatsächlichen 


\section{Clusteranalyse}

Verlauf nur teilweise nachbilden ( $\mathrm{vgl}$. Verlauf des 0.99 -Quantil) und somit ergeben sich große Unterschiede zwischen den berechneten und den repräsentativen Temperaturzuständen. Eine deskriptive Vereinfachung der Realität ist mit der Anwendung kaum möglich.

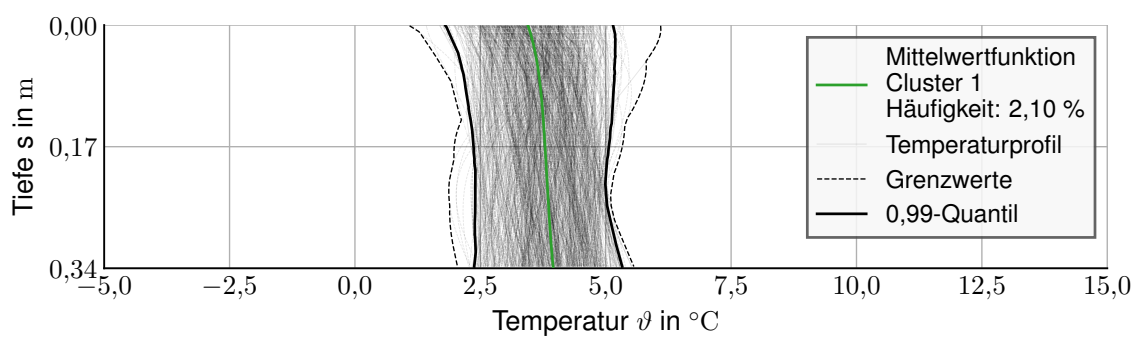

(a) Mittelwertfunktionen

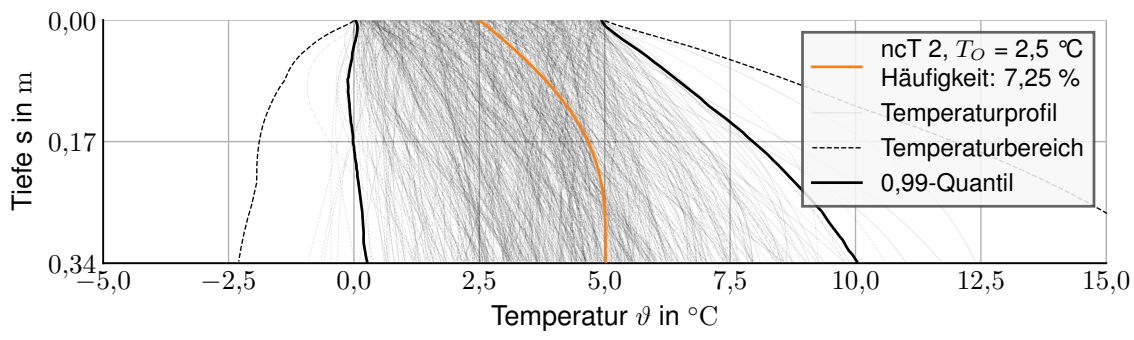

(b) normierte charakteristische Temperaturprofile

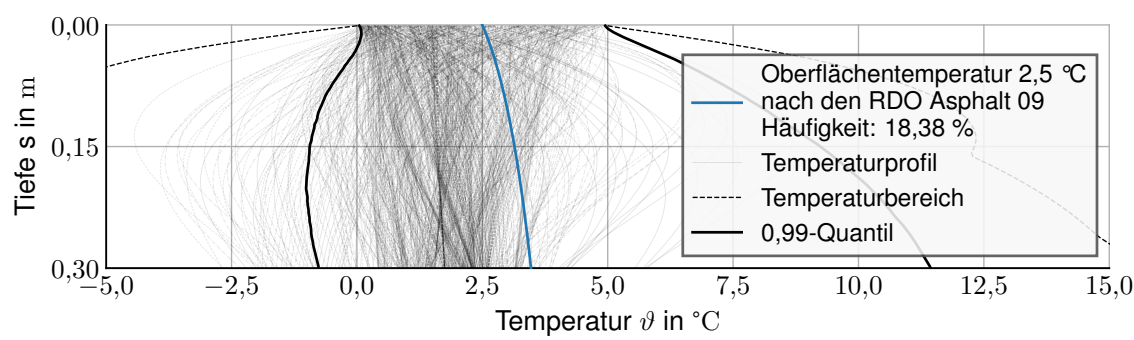

(c) nach RDO Asphalt (2009)

Abbildung 5.7

Darstellung der Funktionsverläufe sowie zufällig ausgewählter ,jedoch zugehöriger Temperaturprofile, Betrachtungszeitraum 2020 - 2049

In Abbildung 5.8 wurde die Abweichung zwischen den repräsentativen Temperaturprofilen aller Klassen bzw. Cluster zu den zugeordneten Temperaturbedingungen innerhalb der StraBenkonstruktion dargestellt. Der Vergleich wurde getrennt nach dem üblichen Verfahren und dem Verfahren zur Bestimmung der Mittelwertfunktionen durchgeführt. Die Funktionsverläufe wurden dabei in $1 \mathrm{~cm}$ große diskrete Teilstücke unterteilt und die jeweilige absolute Differenz zwischen dem Mittelwert und allen zugehörigen Temperaturprofilen berechnet. 
Extreme Abweichungen wurden bei den drei Verfahren nicht berücksichtigt, da hier das 0.99 -Quantil der Abweichungen dargestellt wird.

Aus den Grafiken (Abbildung 5.8a) ist ersichtlich, dass die Mehrheit der Cluster der Mittelwertfunktionen eine Abweichung kleiner $6 \mathrm{~K}$ aufweisen. Nur wenige Cluster überschreiten eine Abweichung größer als 8,00 K. Der Maximalwert liegt mit weniger als 9,00 K in einem tolerierbaren Bereich. Die resultierende Abweichung in die Tiefe der Konstruktion ist über alle Asphaltschichten als gleichwertig anzusehen.

Der direkte Vergleich mit den Verfahren nach den RDO Asphalt (2009) und Kayser (2007) in Abbildung 5.8 ( $b$ und c) zeigt, dass nahezu alle Klassen dieser Verfahren eine signifikant höhere Abweichung aufweisen. Die berechnete Differenz zwischen den realen Temperaturprofilen und den dimensionierungsrelevanten Temperaturverläufen ist zum Teil erheblich. In vielen Fällen wurde eine Abweichung von deutlich mehr als $20^{\circ} \mathrm{C}$ festgestellt. Aus den Daten konnte weiterhin abgeleitet werden, dass die statistischen Temperaturen in der Asphaltdeckschicht relativ geringe Abweichungen zu den simulierten Werten aufweisen. Mit Zunahme der Tiefe in der Straßenkonstruktion nehmen die Unsicherheiten aber deutlich zu.

Der Einfluss dieser Abweichungen kann mithilfe eines fiktiven Straßenaufbaus exemplarisch dargestellt werden. Die beispielhaft gewählte Straßenkonstruktion besteht aus drei Asphaltschichten, wobei die Gesamtdicke der Asphaltdeck-, Asphaltbinder- und Asphalttragschicht mit $34 \mathrm{~cm}$ festgelegt wurde. Für die Referenzvariante wurde eine konstante Temperatur von $20^{\circ} \mathrm{C}$ im gesamten Aufbau gewählt. Es wurden weitere lineare Temperaturprofile definiert, wobei die Oberflächentemperaturen konstant sind und sich die Temperatur in der Tiefe ändert. Die definierten Temperaturen an der Unterseite der Asphalttragschicht liegen im Bereich zwischen $10{ }^{\circ} \mathrm{C}$ und $30^{\circ} \mathrm{C}$ (Abbildung 5.9).

Für jeden Temperaturverlauf wurde eine eigenständige Dimensionierungsrechnung mit konstanten Eingangsparametern durchgeführt. Der berechnete Ermüdungsstatus entspricht einem Zustand in der Betrachtung der Dauerhaftigkeit von Straßenkonstruktionen und die ermittelten Teilschädigungen wurden dem Referenzwert gegenübergestellt (Abbildung 5.9a). Eine Änderung der Temperatur in der Asphalttragschicht um $-10,00 \mathrm{~K}$ hat eine Reduzierung des Ermüdungsstatus auf ca. 20,00 \% zur Folge. Eine Veränderung der Temperatur an der Unterseite der Asphalttragschicht um 10,00 K führt dazu, dass der Schädigungsanteil auf ca. 800,00\% gegenüber dem Referenzwert steigt.

Analog zu diesem Vorgehen wurde der Einfluss einer ungenauen Zuordnung der Temperaturen im Bereich der Asphaltdeckschicht bewertet (Abbildung 5.10). Die Oberflächentemperatur wurde dafür im Bereich zwischen $30,00^{\circ} \mathrm{C}$ und $50,00{ }^{\circ} \mathrm{C}$ variiert, wobei die Temperatur an der Unterseite der Asphalttragschicht als konstant festgelegt wurde. Auf Basis der Temperaturverläufe wurde der Ermüdungsstatus an der Unterseite der Asphalttragschicht ermittelt. Die resultierende Abweichung ist deutlich geringer als die im vorhergehenden Beispiel.

Daraus kann abgeleitet werden, dass eine möglichst geringe Abweichung zwischen realen Temperaturbedingungen und den statistischen Mittelwerten auftreten sollte. Eine hohe 

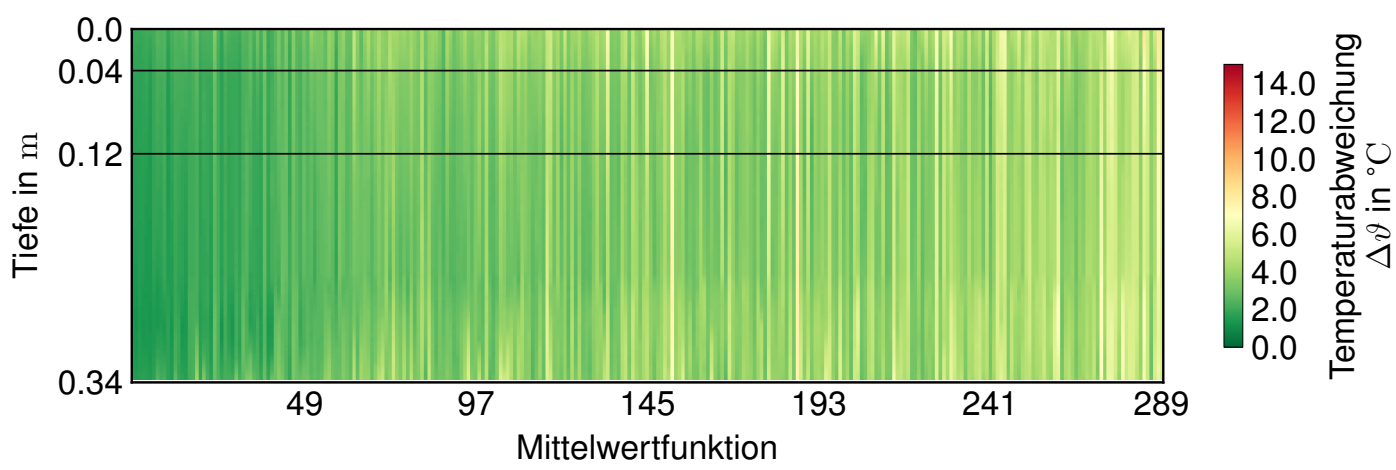

(a) Mittelwertfunktionen
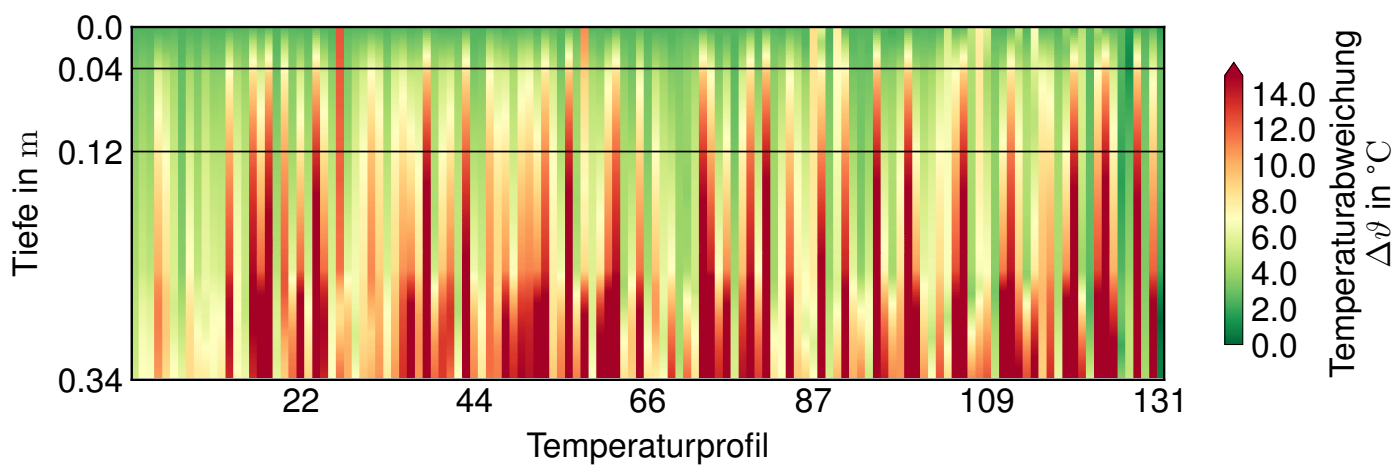

(b) normierte charakteristische Temperaturprofile

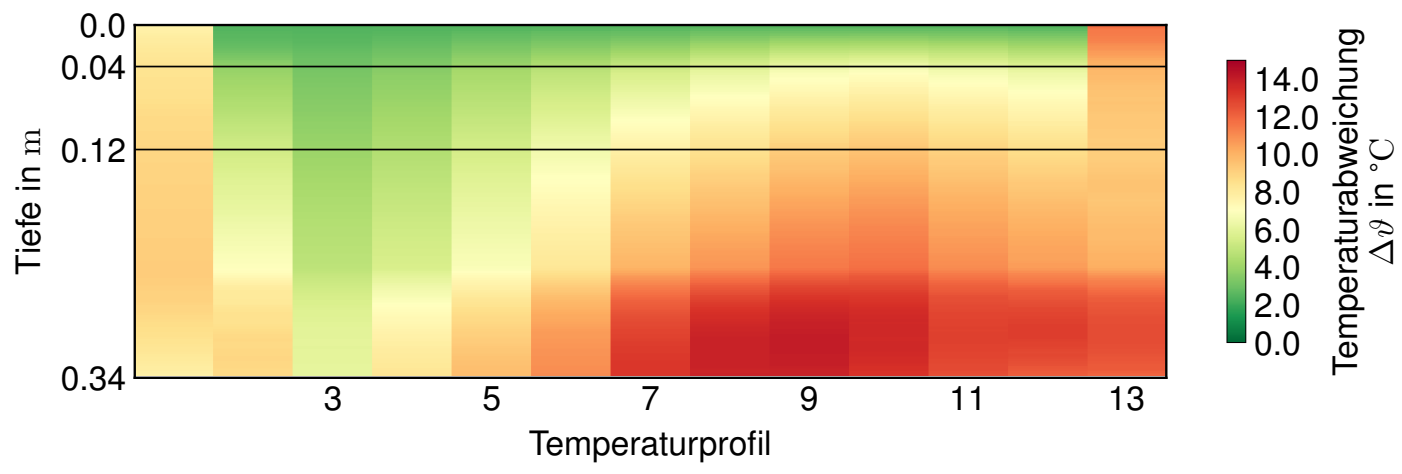

(c) nach RDO Asphalt (2009)

Abbildung 5.8

Abweichungen zwischen den Klassenmittelwerten (Temperaturprofilen) und allen zugehörigen Tempertaturbedingungen, 0,99 Quantil 


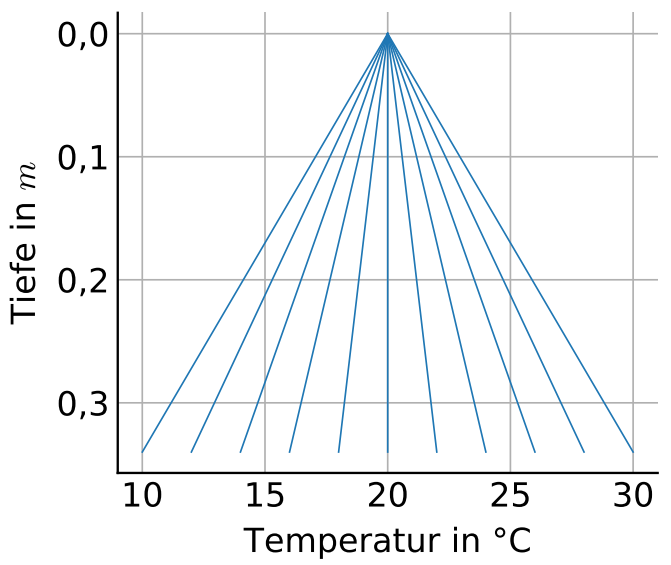

(a) Temperaturprofile

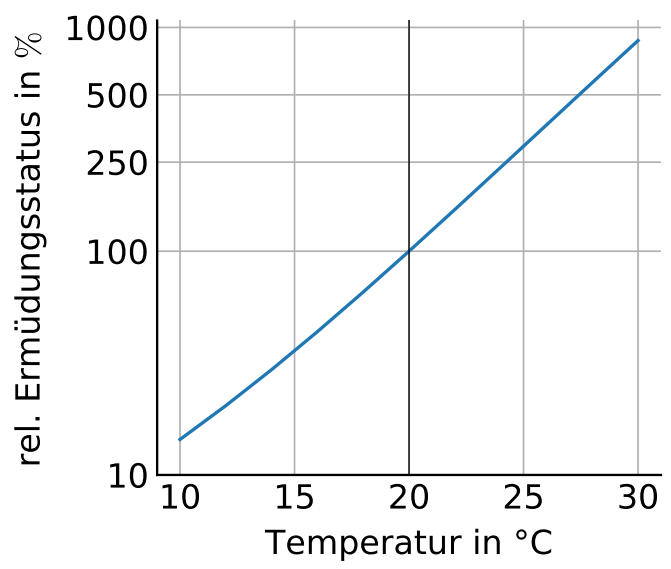

(b) Schädigungspotenzial

Abbildung 5.9

Eingangsdaten und Ergebnisse einer Beispielrechnung zur Ermittlung des Einflusses der Temperatur auf die Dauerhaftigkeit von Straßenkonstruktionen; Parameterstudie mit hohen Abweichungen innerhalb der Asphalttragschicht

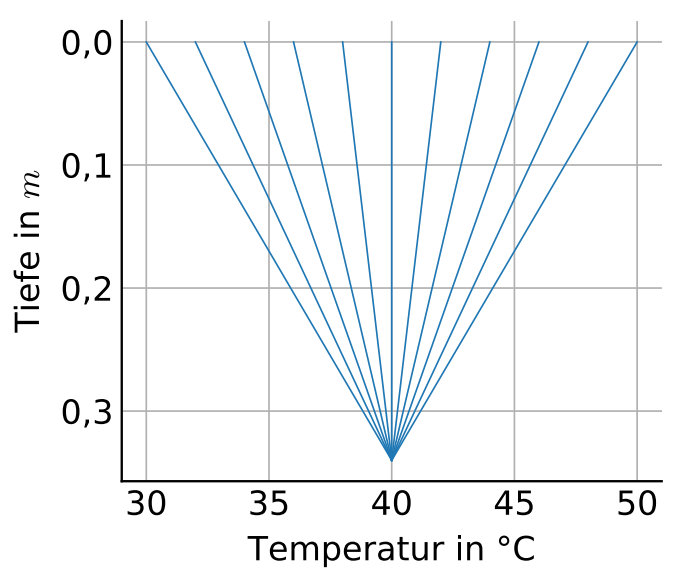

(a) Temperaturprofile

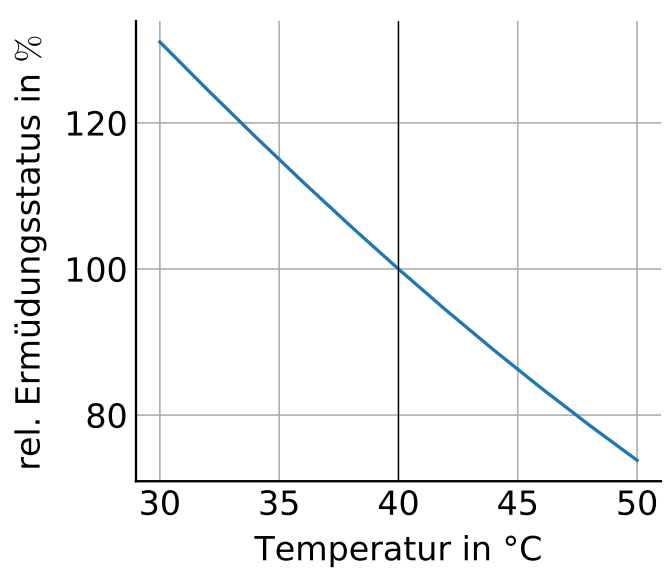

(b) Schädigungspotenzial

Abbildung 5.10

Eingangsdaten und Ergebnisse einer Beispielrechnung zur Ermittlung des Einflusses der Temperatur auf die Dauerhaftigkeit von Straßenkonstruktionen; Parameterstudie mit hohen Abweichungen innerhalb der Asphaltdeckschicht 


\section{Clusteranalyse}

Abweichung in den tieferen Schichten ist besonders kritisch für den Nachweis auf Ermüdungsrissbildung. Eine möglichst exakte Bestimmung der Temperaturbedingungen ist daher extrem wichtig für die Güte von Dimensionierungsrechnungen.

Die Reduktion der Anzahl an Temperaturbedingungen durch Anwendung des Clusterverfahrens zur Berechnung der Mittelwertfunktionen stellt eine legitime Möglichkeit dar, die Vielzahl an potenziellen Temperaturbedingungen innerhalb einer Straßenbefestigung auf eine praktikable Menge zu verringern. Dabei werden charakteristische Merkmale wie die thermophysikalischen Materialeigenschaften sowie die lokalen klimatischen Bedingungen berücksichtigt. Dies stellt sicher, dass die real vorhandenen thermischen Bedingungen innerhalb der zu untersuchenden Straßenbefestigung im Dimensionierungsverfahren mit möglichst geringen Abweichungen berücksichtigt werden können. Weiterhin kann festgestellt werden, dass lokale Bedingungen und die Materialeigenschaften der Baustoffe von Straßenbefestigungen so unterschiedlich sind, dass eine Standardisierung der Temperaturbedingungen für eine vereinfachte Analyse kaum möglich ist. Die Anwendung eines vereinfachten Verfahrens führt mit einer sehr hohen Wahrscheinlichkeit zu stark abweichenden Ergebnissen. Diese Abweichungen haben wiederum sehr hohe Unsicherheiten bei der Ermittlung des Schädigungspotenzials von Straßenbefestigungen durch unterschiedliche Fahrzeuge zur Folge. 


\section{Klimafolgen für Asphaltbefestigungen}

\subsection{Motivation}

Die Daten des verwendeten Klimamodells zeigen, dass langfristig mit ungünstigen Veränderungen der Randbedingungen zu rechnen ist (Abschnitt 4.3). Der projizierte Anstieg der Lufttemperatur im Mittel von mehr als $3 \mathrm{~K}$ und die Zunahme des Strahlungswärmestroms haben einen direkten Einfluss auf die thermischen Zustände von Straßenkonstruktionen.

In einer ersten Studie wurde bereits der Einfluss auf wenige ausgewählte Gebiete für Straßenbefestigungen in Asphaltbauweise und Betonbauweise detektiert (Clauß, Kayser und Wellner, 2017). Darin konnte jedoch festgestellt werden, dass die in den Regelwerken vorgeschlagenen Temperaturprofile und Häufigkeiten deutliche Abweichungen zu den simulierten Ergebnissen aufweisen.

Die Fragestellungen, die sich aus der Studie ergeben, sind vielfältig. Unter anderem ist unklar, ob einzelne Bundesländer und Landkreise in Deutschland gesondert auf die zu erwartenden klimatischen Änderungen reagieren müssen. Weiterhin ist von Interesse, ob die klimatischen Bedingungen das Ermüdungsverhaltens von Asphaltbefestigungen negativ beeinflusst oder ob zusätzlich auch ein höheres Potenzial gegenüber der Entwicklung von Spurrinnen besteht.

Zu diesem Zweck wurde in dieser Arbeit für die Landkreise der gesamten Bundesrepublik Deutschland eine Analyse beruhend auf dem vorstehend beschriebenen erweiterten Clusterverfahren zur Bestimmung der Mittelwertfunktionen der Temperaturprofile in StraBenkonstruktionen durchgeführt. Die einzelnen Arbeitsschritte und Ergebnisse sind in den vorhergehenden Abschnitten ausführlich aufgezeigt. Das beschriebene Verfahren ermöglicht es, die klimatischen Bedingungen innerhalb der untersuchten Straßenkonstruktionen für den Zeitraum von 1960 bis 2100 für die einzelnen Landkreise zu berechnen.

Die Ergebnisse der numerischen Simulationen wurden in diskrete Abschnitte von je 30 Jahren mit einem Abstand von zehn Jahren eingeteilt (z. B. 2000 bis 2029 oder 2010 bis 2039) und die Dauerhaftigkeit von Straßenbefestigungen in Asphaltbauweise für alle Perioden berechnet. Das Dimensionierungsverfahren nach den RDO Asphalt (2009) unterscheidet zwei Kriterien für die Nachweisführung der Asphaltschichten. Zum einen kann die Entwicklung der Ermüdungsrissbildung in Abhängigkeit der Eingangsparameter aus der Beanspruchung berechnet werden und zum anderen erfolgt die Bewertung der Entwicklung von Spurrinnen. Beide Faktoren wurden für eine Analyse der Vulnerabilität der deutschen Verkehrsinfrastruktur in Asphaltbauweise verwendet. 


\section{Klimafolgen für Asphaltbefestigungen}

In diesem Kapitel werden die Auswirkungen der betrachteten klimatischen Veränderungen ausgewertet und mögliche Folgen für Asphaltbefestigungen abgeleitet.

\subsection{Risiko Ermüdungsrissbildung}

In diesem Abschnitt werden die Ergebnisse der Berechnungen auf Basis des Kriteriums auf Ermüdungsrissbildung von Asphaltbefestigungen im Rahmen des prognostizierten Klimawandels analysiert. Die notwendigen Arbeitsschritte für das Verfahren sind detailliert in Abschnitt 3.1 erläutert.

Die Sensitivität der Nutzungszeiträume von Straßen in Asphaltbauweise kann mittels Serienrechnungen in Abhängigkeit der regionalen Klimaparameter untersucht werden. Das zugrunde gelegte Modell ist eine Kopplung eines thermischen und mechanischen Modells, wobei die Einzelrechnungen sequenziell ausgeführt wurden.

In dieser Arbeit wurden die lokalen Klimaparameter des Klimamodells REMO-UBA mit dem Emissionsszenario A1B verwendet (Jacob, 2005a). Die klimatischen Eingangsparameter wurden für jeden Landkreis spezifisch berechnet (siehe Abschnitt 4.3). Dabei wurden Zeitreihen mit den jeweiligen Flächenmittelwerten aller einzelnen Parameter bestimmt und für die individuellen thermischen Berechnungen als Eingangsparameter verwendet. Alle weiteren Parameter zur Berechnung des Nutzungszeitraums von Straßenkonstruktionen nach den Verfahren der RDO Asphalt (2009) wurden für alle Landkreise als konstant angenommen. Dies ermöglicht es, die Auswirkungen der klimatischen Parameter und deren Änderungen isoliert zu bewerten.

Nach der Durchführung der Berechnungen der thermischen Beanspruchungen für alle Landkreise, wurden die Ergebnisse für jede einzelne Simulation nach dem Verfahren der Mittelwertfunktionen analysiert (Kapitel 5) und relevante Temperaturbedingungen, also der Verlauf der Temperaturprofile und der zugehörigen Häufigkeiten, für die nachfolgenden Dimensionierungsberechnungen individuell bestimmt.

Auf Basis der Simulationen der thermischen Zustände und der daraus bestimmten Mittelwertfunktionen wurden die mechanischen Beanspruchungen für alle Kombinationen von Temperaturprofilen und Verkehrslasten innerhalb der fiktiven Straßenkonstruktion berechnet und der Nutzungszeitraum in Abhängigkeit vom Beginn der Verkehrsfreigabe bestimmt. Die Beanspruchungen innerhalb der Periode 1960 bis 2100 wurden berücksichtigt und in die Analysen mit einem zeitlichen Abstand von 10 Jahren ab 1960 durchgeführt. Für jeden Standort wurden somit 12 Einzelzeiträume bewertet.

Die Eingangswerte für die mechanischen Berechnungen sind in Tabelle 6.1 und die verwendeten Materialkennwerte sind in Tabelle 6.2 aufgeführt. In den Serienrechnungen wurden die Eingangsparameter und die Materialkennwerte für jede Variante genutzt. Dies ermöglicht die Vergleichbarkeit der Ergebnisse im Bundesgebiet. 
Tabelle 6.1

Eingangswerte für die Dimensionierungsrechnungen

\begin{tabular}{|c|c|c|c|}
\hline Parameter & & Wert & Quelle \\
\hline Nutzungszeitraum & $\mathrm{N}$ & 30 Jahre & \\
\hline $\begin{array}{l}\text { Achsübergänge } \\
\text { mittlerer jährlicher Zuwachs in- } \\
\text { nerhalb einer Periode }\end{array}$ & $A$ & $\begin{array}{l}\text { 100,00 Mio. Aü } \\
3,0 \%\end{array}$ & \\
\hline Achslastklasse & & BAB-Fernverkehr & RDO Asphalt, 2009, Tabelle A 1.1 \\
\hline Shiftfaktor & SF & 1500 & RDO Asphalt, 2009, S. 26 \\
\hline Sicherheitsbeiwert & $\mathrm{F}$ & 2,1 & RDO Asphalt, 2009, Tabelle 8.2 \\
\hline
\end{tabular}

Tabelle 6.2

Parameterdarstellung der Materialkennwerte der Basisvariante nach (Clauß u. a., 2019)

\begin{tabular}{lccccccc}
\hline Material & \multicolumn{4}{c}{ Masterfunktion } & \multicolumn{3}{c}{ Ermüdungsfunktion } \\
& $\mathrm{E}_{\min }$ & $\mathrm{E}_{\max }$ & $\mathrm{b}_{0}$ & $\mathrm{~b}_{1}$ & $\mathrm{~m}$ & $\mathrm{C}_{1}$ & $\mathrm{C}_{2}$ \\
\hline Asphaltdeckschicht & 0 & 18527,51 & $-0,94$ & 2,43 & 18000,00 & 1,28 & $-3,40$ \\
Asphaltbinderschicht & 0 & 34456,97 & $-0,74$ & 1,35 & 19569,00 & 0,71 & $-3,84$ \\
Asphalttragschicht & 0 & 25281,45 & $-0,72$ & 1,41 & 18000,00 & 1,38 & $-3,35$ \\
\hline
\end{tabular}

Bedingt durch die Vielzahl an notwendigen Berechnungen wurde ein mechanisches Modell auf Basis der Mehrschichtentheorie verwendet. Für die Ermittlung des Schädigungspotenzials wurden die horizontalen Dehnungen an der Unterseite der Asphalttragschicht in der Lastachse ausgewertet.

Die regional unterschiedlichen klimatischen Bedingungen haben einen direkten Einfluss auf das thermische Verhalten von Straßenkonstruktionen (Abschnitt 4.4). Die beschriebene Analysemethodik ermöglicht es, diesen Einfluss zu berücksichtigen. Die Änderung des lokalen Klimas wird je Variante und Zeitraum unter Verwendung von statistisch bestimmten Temperaturverläufen (Mittelwertfunktionen) und deren stündlichen Häufigkeitsverteilungen berechnet. Die weiteren Parameter, wie die Anzahl der Achsübergänge oder die Sicherheitsbeiwerte, wurden für alle Varianten als konstant festgelegt. Das gewählte Vorgehen ermöglicht einen direkten Vergleich zwischen den unterschiedlichen Verfahren und Regionen.

In Abbildung 6.1 sind die Ergebnisse der Serienrechnungen auf Basis der Mittelwertfunktionen dargestellt. Die Abbildung enthält exemplarisch vier Zeiträume, wobei die Verkehrsfreigaben ab 2000, 2020, 2040 und 2070 aufgeführt sind. Alle Darstellungen der unterschiedlichen Untersuchungszeiträume sind dem Anhang C.1 beigefügt. Die Ergebnisse sind auf den Flächenmittelwert für die Variante mit der Verkehrsfreigabe ab 2020 normiert.

Die Verteilung der Ergebnisse zeigt, dass zukünftig mit einem erheblichen Einfluss des Klimawandels auf die Nutzungszeiten von Asphaltbefestigungen gerechnet werden muss. Die Simulationsergebnisse ergeben einen kontinuierlichen Abfall der resultierenden Nutzungszeiträume, wobei in einigen Regionen eine Verringerung um mehr als $50 \%$ detektiert werden kann. 

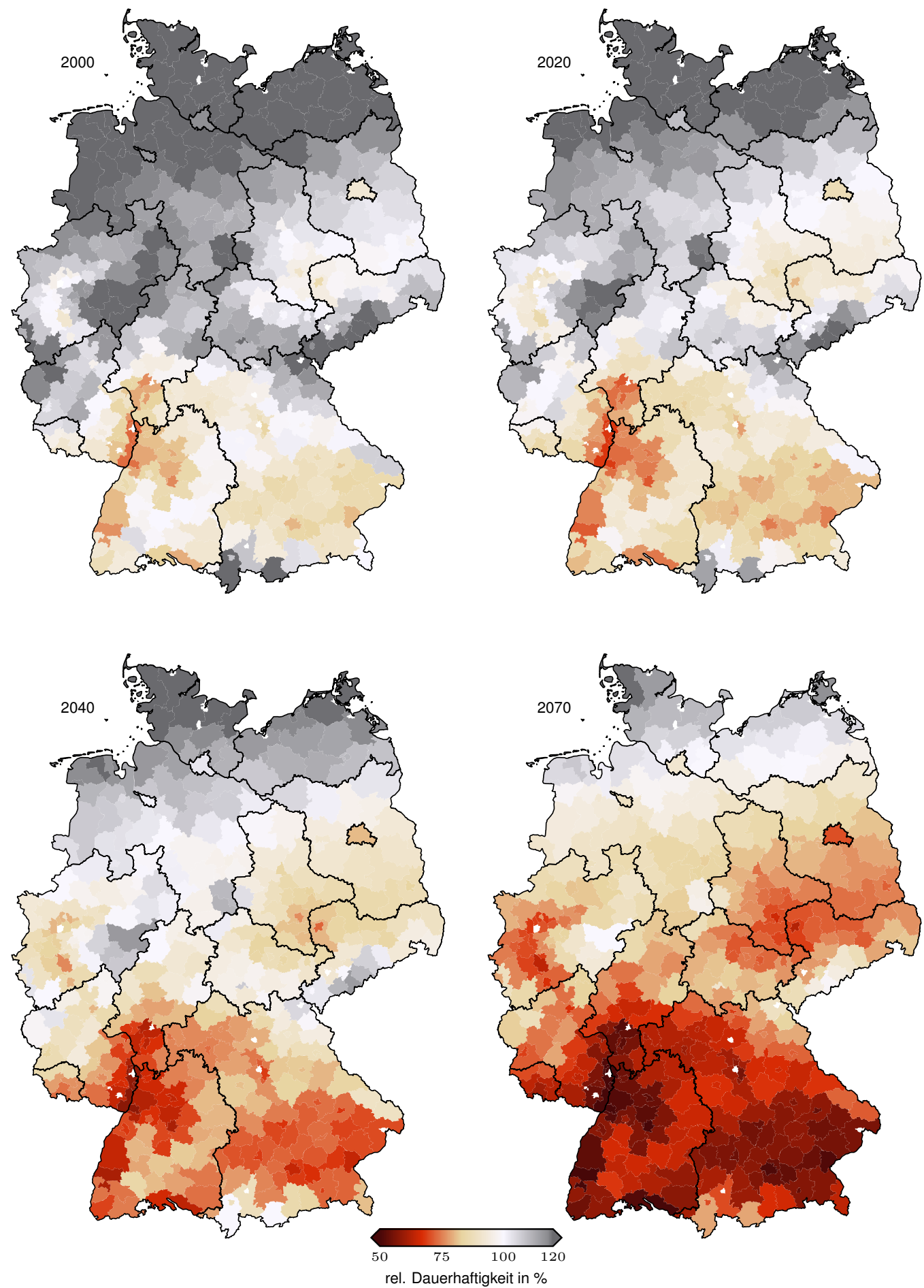

Abbildung 6.1

Relative Dauerhaftigkeit der Asphalttragschicht für das Gebiet Deutschland auf Basis der Mittelwertfunktionen; normiert auf den über die Fläche bestimmten Mittelwert aller Varianten mit der Verkehrsfreigabe ab 2020 
Die Daten der Klimaprognosen haben für die Zeiträume von 1960 bis ca. 2020 zeitlich konstante Bedingungen gezeigt (vgl. Abschnitt 4.4). Dies kann auch in den Ergebnissen der Simulationen des mechanischen Verhaltens der Straßenbefestigung nachgewiesen werden. Der Vergleich der Verteilungen der Ergebnisse über das Bundesgebiet in 2000 und 2020 ergibt nur geringe Unterschiede. In süd-westlichen Gebieten ergeben sich etwas höhere Beeinflussungen der Dauerhaftigkeiten infolge der klimatischen Bedingungen, jedoch sind die Ergebnisse relativ ähnlich. Der Vergleich zeigt für eine Verkehrsfreigabe ab dem Jahr 2040 bereits eine deutliche Verringerung der Nutzungszeiträume. Diese sinken im gesamten Bundesgebiet ab, wobei davon auszugehen ist, dass Süd- und Mitteldeutschland besonders stark betroffen sein werden. Bis zum Ende des Betrachtungszeitraums (Verkehrsfreigabe ab 2070) schreitet dieser Prozess entsprechend dieser Prognosen noch deutlich stärker voran. Der gesamte südliche Raum unterliegt einer Reduktion der Nutzungszeiten um mindestens $25 \%$. Für einige Landkreise konnte sogar ein Abfall der Nutzungszeiträume auf die Hälfte gegenüber der über die Fläche gemittelten Nutzungszeit mit einer Verkehrsfreigabe im Jahr 2020 festgestellt werden.

Ein signifikanter Einfluss kann auch für den Großraum Berlin mit Teilen von Brandenburg und der Altmark festgestellt werden. Die Küstenregionen unterliegen ebenfalls einer kontinuierlichen Verkürzung der Nutzungszeiträume.

Die berechnete Dauerhaftigkeit von Straßenkonstruktionen innerhalb einer Zeitperiode variiert zwischen den einzelnen Landkreisen. In Abbildung 6.2 ist diese Varianz in Abhängigkeit von der Verkehrsfreigabe für drei Zeiträume dargestellt. Die Normierung erfolgt, analog zu den vorherigen Analysen, über die relative Abweichung zum Flächenmittelwert des Bundesgebietes für die Periode mit der Verkehrsfreigabe ab 2020. Aus der Darstellung ist ersichtlich, dass die Varianz für das Jahr 2020 im Bereich von ca. $60 \%$ bis ca. 150\% liegt. Der Flächenschwerpunkt befindet sich im mittleren Bereich bei ca. 100\%.

Vergleicht man die Werte für die Verkehrsfreigabe ab 1960, so ergibt sich eine Verschiebung hin zu höheren Nutzungszeiträumen. Die Varianz und der Verlauf der Funktion ähneln den Werten von 2020, es wurde jedoch ein Versatz um ca. $20 \%$ festgestellt.

Die Verteilung der zukünftigen Parameter (Betrachtungszeitraum ab 2070) ist stark in den negativen Bereich verschoben. Im ungünstigsten Fall ergibt sich eine Reduktion der Nutzungszeit auf unter $40 \%$ im Vergleich zur Freigabe in 2020. Die Linkslastigkeit des Verlaufes zeigt, dass die klimatischen Veränderungen einen nicht linearen Einfluss auf die Berechnungsergebnisse haben. Auf Basis der Ergebnisse ist eine signifikante Verringerung der Dauerhaftigkeiten von Straßenbefestigungen in Asphaltbauweise in einem Großteil des Bundesgebietes sehr wahrscheinlich. Eine Anpassung der Asphalte an die Folgen des Klimawandels muss erfolgen.

Die Gruppierung der Einzelergebnisse nach Bundesland und Zeit ermöglicht die Bewertung der Vulnerabilität auf Landesebene. Für jedes Bundesland kann anhand der Einzelwerte die mittlere Entwicklung abgeleitet werden. Aus den Ergebnissen der Berechnung kann eine 


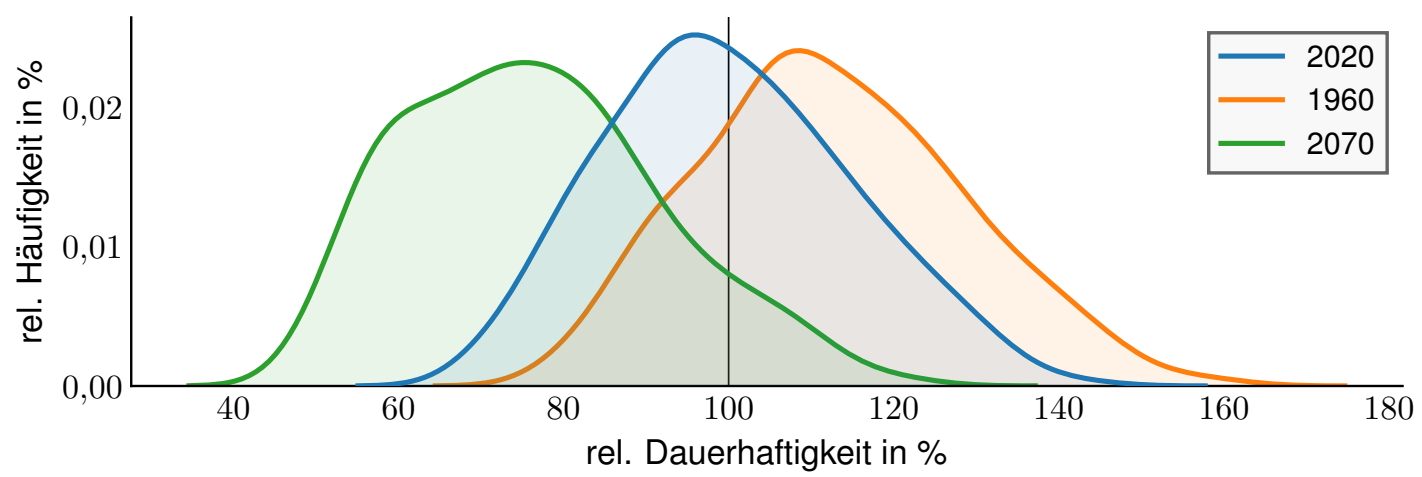

Abbildung 6.2

Häufigkeitsverteilung der prognostizierten Dauerhaftigkeit für alle Landkreise normiert auf den Bundesdurchschnitt für die Periode ab dem Jahr 2020

solche Übersicht auch problemlos bis auf die Ebene von Landkreisen zusammengestellt werden.

Die Ergebnisse in Abbildung 6.3 zeigen, dass die Infrastruktur in Baden-Württemberg das größte Risiko gegenüber den Folgen des projizierten Klimawandels aufweist. Im Gebiet dieses Bundeslandes sinken die prognostizierten Nutzungszeiträume am stärksten. In Bayern, Berlin, Hessen, Rheinland-Pfalz und dem Saarland erfolgt eine etwas abgeflacht Entwicklung, jedoch muss auch in diesen Bundesländern von einer deutlichen Verstärkung der klimabedingten Schädigungen an der Verkehrsinfrastruktur in Asphaltbauweise gerechnet werden.

Die Dimensionierungsrechnungen nach dem Verfahren der Mittelwertfunktionen zeigen differenzierte Abweichungen zwischen den Regionen. Die Berechnungen ergeben, dass unter Beachtung von gleichen Verkehrszahlen und Randparametern die klimabedingten Schädigungen an der Verkehrsinfrastruktur in Asphaltbauweise deutlich geringer ausgefallen sind. Die Ergebnisse dieser Arbeit belegen, dass die klimatischen Bedingungen der Vergangenheit einen begünstigenden Einfluss auf die Nutzungszeiträume hatten. Überlagert man den klimatischen Effekt mit den zum Teil deutlich geringeren Verkehrszahlen der Vergangenheit, so kann angenommen werden, dass aktuelle Verkehrsflächen ein deutlich erhöhtes Risiko gegenüber klimainduzierten Schädigungen aufweisen. Die zeitliche Änderung der Daten ergibt, dass die Vulnerabilität der Verkehrsinfrastruktur in Deutschland stetig steigt. Dies kann bei allen untersuchten Landkreisen und Bundesländern festgestellt werden. 


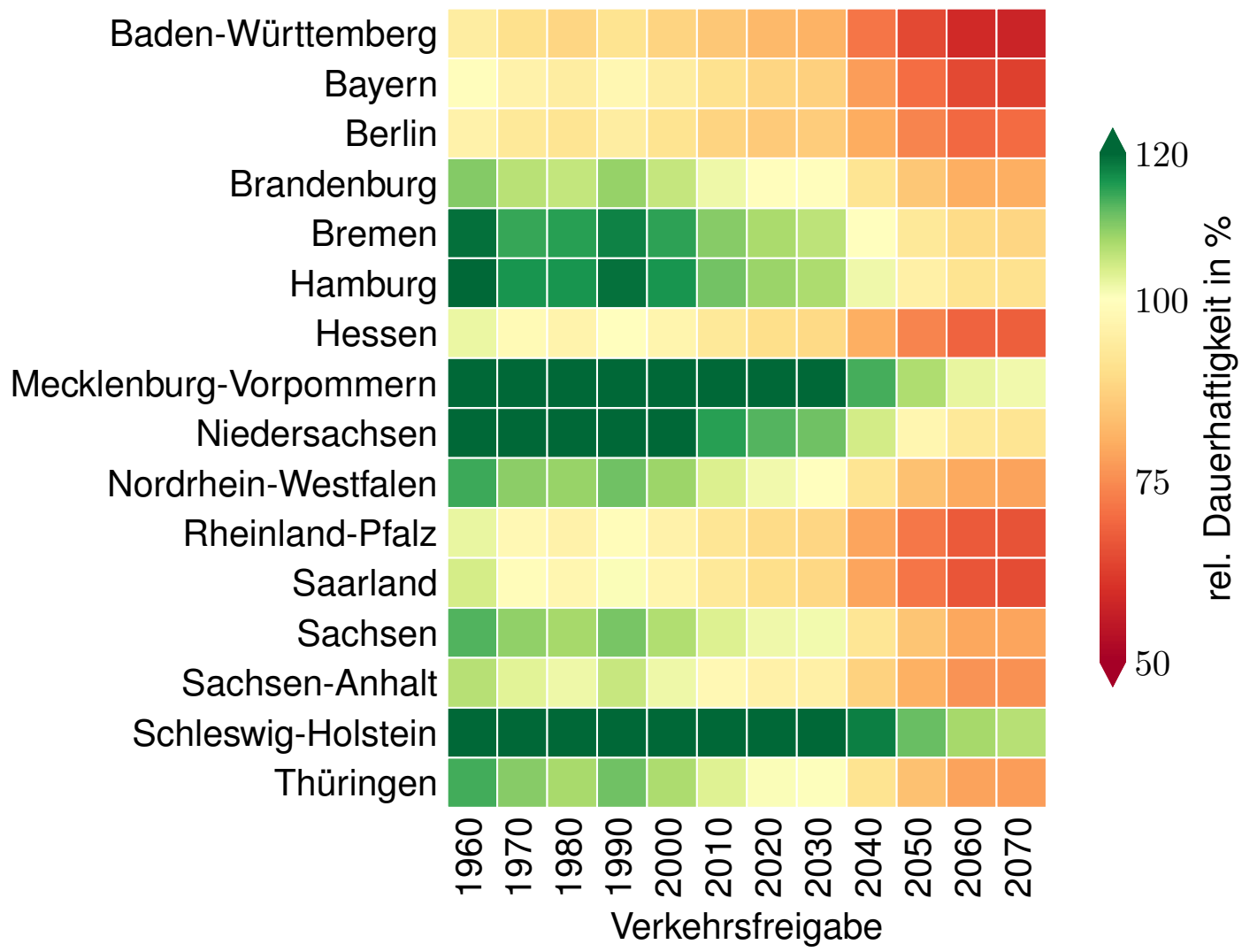

Abbildung 6.3

Ermüdungsstatus in Abhängigkeit des Bundeslandes und der Verkehrsfreigabe

\subsection{Risiko Spurrinnenentwicklung}

Eine weitere sehr wahrscheinliche Folge des Klimawandels betrifft das plastische Verhalten von Asphaltschichten. Die Erhöhung der Temperaturen innerhalb der Konstruktionen führen zu einer Abminderung der Steifigkeit von Asphalten und somit steigt auch das Verhältnis zwischen den elastischen und den plastischen Anteilen im Materialverhalten von Asphalten. Dies führt zu einem höheren Potenzial gegenüber bleibenden Verformungen. Das Berechnungsverfahren ist im Anschnitt 3.3 aufgezeigt.

Die Entwicklung der Spurrinnentiefe ist nachfolgend dargestellt. In Abbildung 6.4 sind die relativen Veränderungen bezogen auf die mittlere Spurrinnentiefe des Bundesgebietes für den Betrachtungszeitraum 2020 dargestellt.

Die einzelnen Karten zeigen, dass in der Vergangenheit und in der Gegenwart ein nahezu gleiches Risiko gegenüber der Entwicklung der Tiefe von Spurrinnen besteht. Im Norden von Deutschland ergeben die Berechnungen die geringsten Tiefen, wohingegen im Bereich des 

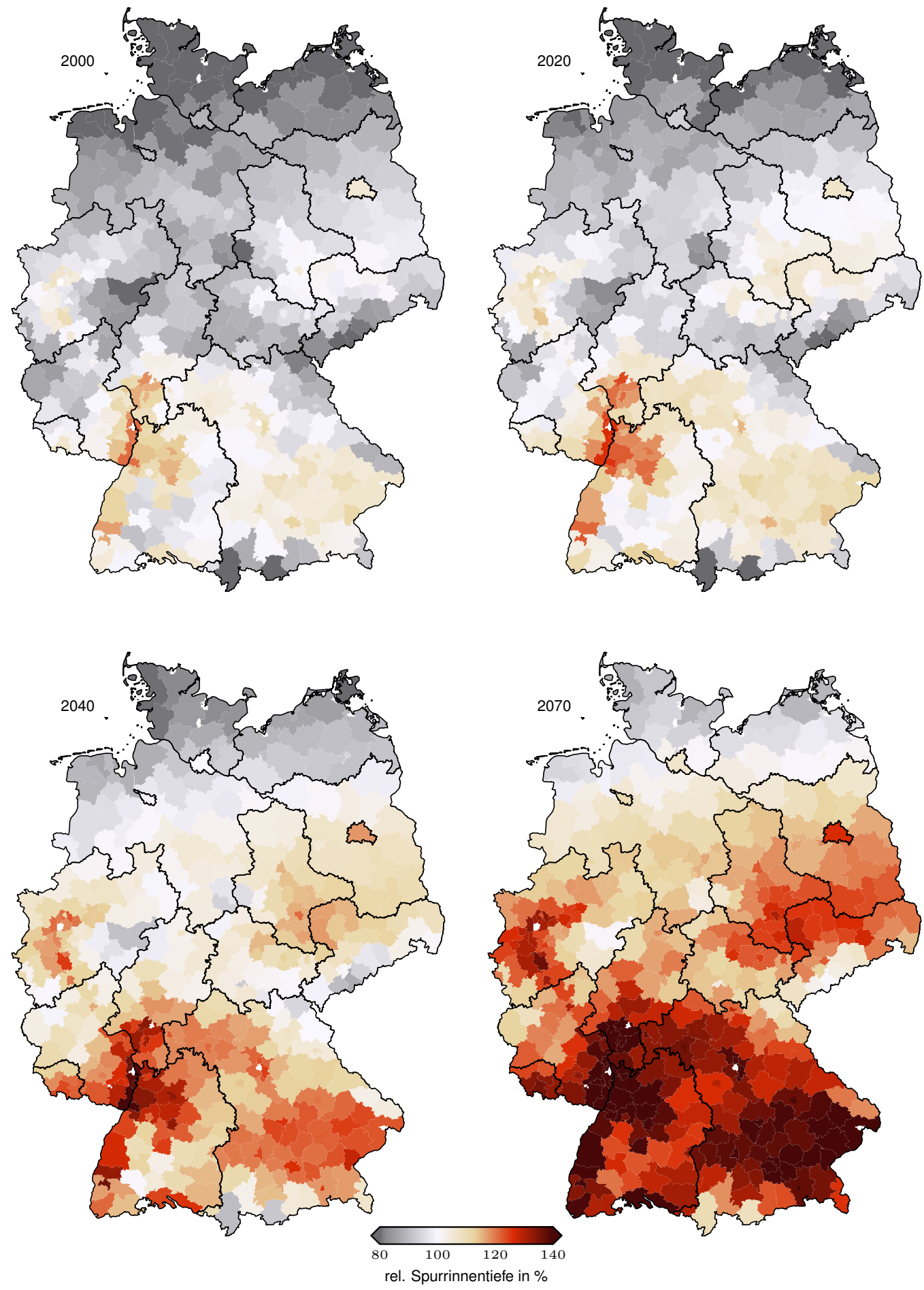

Abbildung 6.4

Ermittelte Spurrinnentiefe für das Gebiet Deutschland auf Basis der Mittelwertfunktionen; normiert auf den Mittelwert aller Landkreise mit Verkehrsfreigabe ab 2020 
Rheins und um Frankfurt am Main größere Beanspruchungen berechnet werden konnten. Mit dem Fortschreiten des Klimawandels erfolgt bundesweit ein Anstieg der resultierenden Spurrinnentiefen. Die Entwicklung nimmt ab 2040 signifikant zu. In Gebieten entlang des Rheins, der Großraum um Frankfurt am Main, Berlin und München sowie Franken, Altmark und Teile von Brandenburg konnte anhand der Prognosen ein erheblicher Anstieg der Spurrinnentiefe festgestellt werden. Für den Betrachtungszeitraum ab 2070 steigt dieser Effekt nochmals deutlich an. Im nahezu gesamten südlichen Bundesgebiet erhöht sich die resultierende Spurrinnentiefe um mehr als $20 \%$, in Teilgebieten sogar um mehr als $40 \%$.

In Abbildung 6.5 ist die Spannweite und die Häufigkeit des Auftretens der relativen Spurrinnentiefe für vier 30-jährige Perioden dargestellt. Die zugrunde gelegten Daten sind auf den Mittelwert der Periode mit der Verkehrsfreigabe ab 2020 normiert. Der Verlauf der Referenzperiode ergibt, dass die relative Spurrinnentiefe für das Bundesgebiet im Bereich zwischen $70 \%$ und $130 \%$ liegen. Der Mittelwert konnte mit ca. $100 \%$ bestimmt werden. Der Vergleich mit den Werten von 1960 zeigt, dass die Werte um ca. 15\% - in Richtung von geringeren Beschädigungen - vermindert sind. Die Form des Verlaufs ähnelt jedoch dem Verlauf von 2020, was einer Verschiebung der mittleren Spurrinnentiefe im Bundesgebiet entspricht. Die zukünftige Entwicklung der Spurrinnentiefe ist von den klimatischen Veränderungen des Klimawandels beeinflusst. Die Varianz der Simulationsergebnisse nimmt 2040 im Vergleich zu 2020 deutlich zu und und dieser Sachverhalt wird bis Ende des Jahrhunderts weiter zunehmen. Mit der Entwicklung der klimatischen Folgen ist auch eine kontinuierliche Zunahme der Spurrinnentiefen im Bundesgebiet zu erwarten. Die Varianz der Simulationsergebnisse weißt darauf hin, dass die Entwicklung nicht homogen erfolgen wird und somit ist eine relevante Schädigung der Straßenbefestigungen durch eine verstärkte Entwicklung von Spurrinnen auch in Gebieten, welche aktuell wenig bis kaum betroffen sind, wahrscheinlich.

Normiert man die Entwicklung der Spurrinnentiefe auf den jeweiligen Wert des Landkreises aus dem Jahr 2020, so ergeben sich die relativen Veränderungen für jeden Landkreis. Dieses Verfahren ermöglicht es, zu prüfen ob der Einfluss des Klimawandels im gleichen Maße für alle Landkreise des Bundesgebietes vorhanden ist. Die Abbildung 6.6 stellt die Ergebnisse in Abhängigkeit vom Jahr der Verkehrsfreigabe dar.

Die vorhergehenden Darstellungen haben gezeigt, dass es zwischen den Landkreisen absolute Unterschiede der berechneten Spurrinnentiefen gibt. Die relativen Veränderungen der einzelnen Landkreise sind tendenziell ähnlich. Zwischen 1960 und 2030 ergeben die Berechnungen nur geringe Abweichungen und die Tiefe der Spurrinnen ändert sich kaum. Die Daten zeigen weiterhin einen sehr geringen Zuwachs innerhalb dieser Periode. Ab 2030 kann ein überproportionaler Zuwachs der Spurrinnentiefe in allen Landkreisen festgestellt werden. Die Werte steigen dabei von ca. $100 \%$ auf über $120 \%$ bis zum Ende des Betrachtungszeitraumes. Einige Landkreise unterliegen einer deutlich höheren Belastung, prognostizierte Zunahmen auf bis zu $140 \%$ wurden detektiert.

Die Entwicklung der Spurrinnentiefe ist stark abhängig von den klimatischen Randbedingungen und führt in Zukunft (ab ca. 2030) zu einer signifikanten Steigerung der Gefährdung durch tendenziell größere Tiefen der Spurrinne. Eine Optimierung der Asphalte gegenüber der 


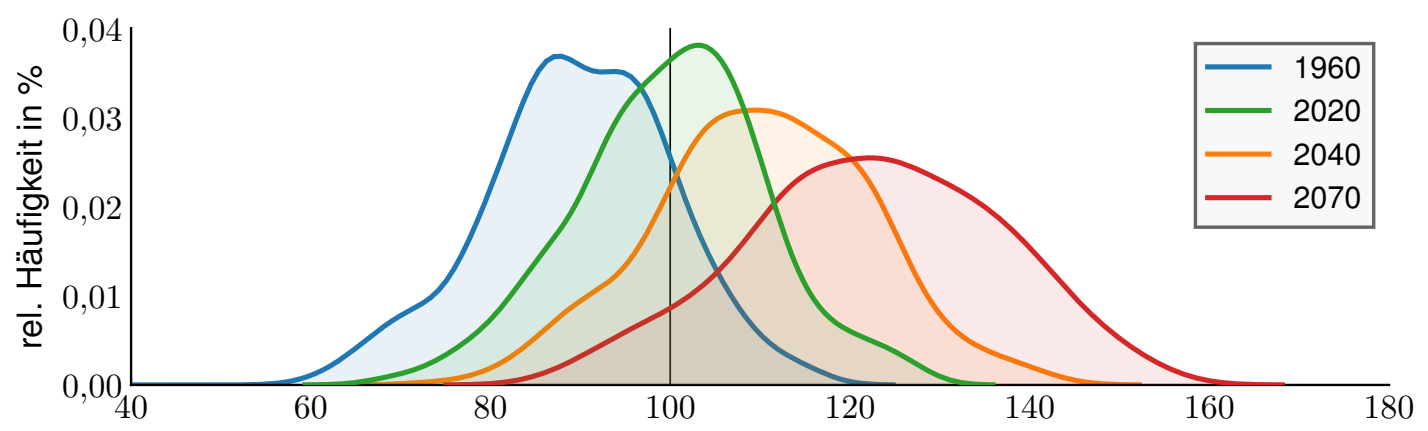

rel. Spurrinnentiefe in \%

Abbildung 6.5

Häufigkeitsverteilung der Spurrinnentiefe für alle Landkreise normiert auf den Bundesdurchschnitt für die Periode ab dem Jahr 2020

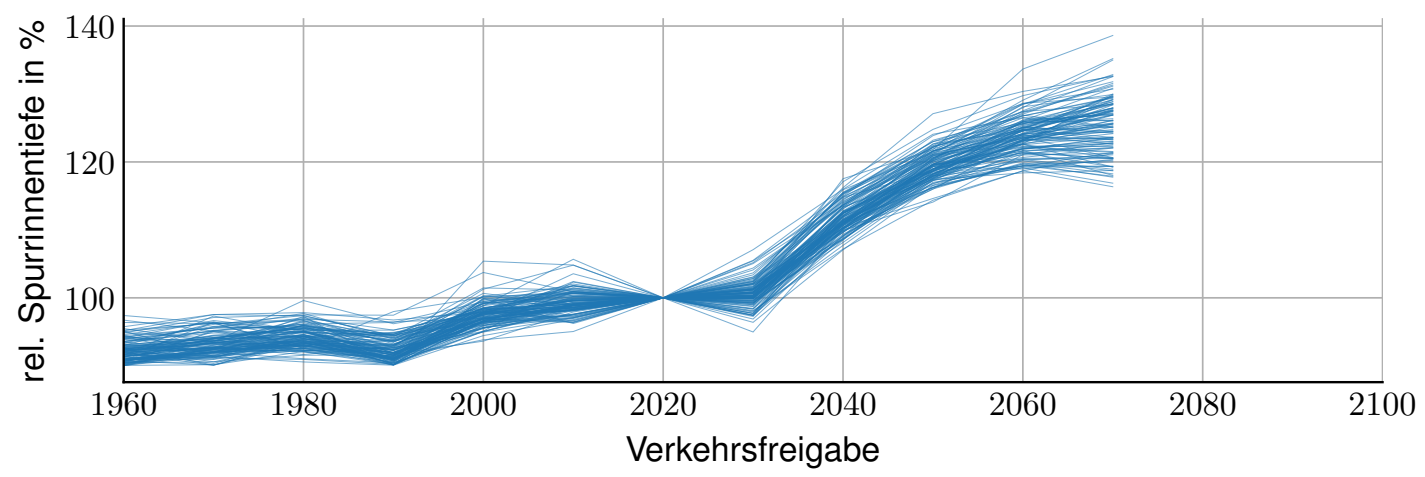

Abbildung 6.6

Relative Spurrinnentiefe in Abhängigkeit der Verkehrsfreigabe, Basiswert ist der Einzelwert jeder Variante für das Jahr 2020 
Anfälligkeit auf plastische Verformung auch für Temperaturen größer als $50^{\circ} \mathrm{C}$ ist zwingend notwendig.

Die Berechnungen hier erfolgten unter Annahme einer konstanten Tagesganglinie des Verkehrs, da die stündlichen Berechnungen sehr umfangreich und zeitaufwändig sind. Die realen Verteilungen haben sehr wahrscheinlich einen deutlich größeren Einfluss auf die Entwicklung der Spurrinnenbildung, da die Verläufe der Mittelwertfunktionen in den Mittagsstunden, also bei relativ hohen Temperaturen, in Kombination mit hohen Verkehrsdichten zu starken Dehnungen in der Konstruktion führen. Dies hat einen deutlichen Einfluss auf die Beanspruchung in den Straßenkonstruktionen und bewirkt eine Steigerung der Spurrinnentiefe.

\subsection{Zusammenfassung}

Mit den in dieser Arbeit durchgeführten Analysen konnte gezeigt werden, dass im gesamten Bundesgebiet Einflüsse der klimatischen Veränderungen prognostiziert werden. Straßenkonstruktionen unterliegen in einem deutlichen Maße den klimatischen Veränderungen. Grundsätzlich muss davon ausgegangen werden, dass eine Reduzierung der Nutzungszeiträume von Straßenbefestigungen erfolgen wird. Weiterhin konnte nachgewiesen werden, dass ein Nord-Süd-Gefälle entsteht. Die südlichen Bundesländer werden verstärkt von den Folgen des Klimawandels beeinflusst werden.

An dieser Stelle muss noch einmal darauf hingewiesen werden, dass in dieser Arbeit die Kriterien auf Ermüdungsrissbildung in der Asphalttragschicht sowie Spurrinnentiefe an der Fahrbahnoberseite als Parameter zur Bewertung verwendet wurden. Aus Gründen der Rechenkapazität konnte das detaillierte Verfahren nach Clauß u. a. (2019), welches die räumlichen Beanspruchungszustände in allen Asphaltschichten berücksichtigt, nicht verwendet werden. Die Ergebnisse aus dem Forschungsprojekt (Clauß u.a., 2019) deuten jedoch an, dass die Beanspruchungen in den Asphaltdeckschichten unter bestimmten Randbedingungen zu einer deutlich früheren Rissbildung führen. Exemplarisch ist dies in einem Fallbeispiel im Abschnitt 7.1 dargestellt.

Die genannten Aspekte führen unweigerlich zu einem erhöhten Bedarf an Instandsetzungskosten, welche Bund und Kommunen zukünftig zum Erhalt der Infrastruktur investieren müssen.

Es wird empfohlen schnellstmöglich auf die Herausforderungen durch die klimatischen Veränderungen zu reagieren. Das Verfahren nach den RStO 12 (2012) kann die entsprechenden kleinräumigen klimatischen Faktoren nicht ausreichend berücksichtigen. Weiterhin berücksichtigen empirische Methoden nur die Datenlage der Vergangenheit. Die Anwendung von numerischen Methoden zur Dimensionierung von Straßenbefestigungen sollte bei Bundesstraßen sowie Autobahnen stets durchgeführt werden. Wichtig ist jedoch auch, dass die mechanischen sowie die thermophysikalischen Materialparameter der eingesetzten Asphalte labortechnisch bestimmt und im Dimensionierungsverfahren berücksichtigt werden. Die 


\section{Klimafolgen für Asphaltbefestigungen}

zukünftige Entwicklung des Klimas sowie des Verkehrs sollte gesondert diskutiert werden. In dieser Arbeit wurde nur ein mögliches Szenario auf Basis des Klimamodells REMOUBA (A1B) durchgeführt. Weitere Untersuchungen und Serienrechnungen sind dringend notwendig.

Außerdem muss an innovativen Bauweisen und Materialien geforscht werden, welche möglichst klimaneutral den zukünftigen Beanspruchungen der Verkehrsströme widerstehen. Eine langfristige und kontinuierliche Förderung ist ratsam. Auch alternative Materialien und Konstruktionsaufbauten sollen verstärkt im Mittelpunkt der Forschung verankert werden. Es gilt zu beachten, dass durch innovative Bauweisen, welche in Deutschland entwickelt werden, auch die Möglichkeit besteht, diese weltweit zu vermarkten. Eine Minderung der Qualität der Verkehrswege sollte dringend verhindert werden, da ein Großteil des Transportes von Gütern auf der Straßeninfrastruktur erfolgt. 


\section{Fallbeispiele}

\subsection{Temperierung von Straßenbefestigungen}

Straßenkonstruktionen unterliegen im Laufe der Nutzungsphase einer Vielzahl an klimatischen Einflüssen. Besonders hohe Temperaturen führen zu einer überproportionalen Schädigung der Straßenbefestigung durch einen schnellen Zuwachs an Ermüdungsrissen oder plastischen Verformungen. Im Rahmen des Forschungsprojektes Untersuchung multifunktionaler StraBenbaumaterialien und Verbundwerkstoffe zur Nutzung solarer Energie und Verbesserung der Dauerhaftigkeit (Clauß u. a., 2019) wurde das Potenzial der Reduzierung der Temperatur in der Straßenkonstruktion durch Nutzung der thermischen Energie geprüft. Die Auskoppelung der Wärme wurde dabei über integrierte Rohrkollektorsysteme realisiert.

Die Funktionen der einzelnen Schichten der Straßenkonstruktion mit Rohrkollektor wurden innerhalb des Projektes differenziert betrachtet. Das Wirkprinzip der Kollektoren besteht darin, durch einen Entzug der thermische Energie an der Oberseite der Straßenkonstruktion die Temperatur in der gesamten Konstruktion zu mindern. Aus dieser Überlegung kann abgeleitet werden, dass die optimale Einbautiefe des Kollektors möglichst oberflächennah sein sollte. Die Asphaltbinderschicht wurde somit als Bettungsschicht für die Kollektoren verwendet. Die Dicke der Bettungsschicht wurde an den Durchmesser der verwendeten Rohre inklusive Überdeckung angepasst. Weiterhin wurde die Dicke der Asphaltdeckschicht auf die technisch minimale Dicke reduziert. Somit können Schädigungen an der Asphaltdeckschicht repariert, gleichzeitig die Beanspruchungen aus dem Verkehr verteilt und die thermische Energie optimal ausgekoppelt werden.

Innerhalb der Bearbeitungsphase des Forschungsprojektes wurden detaillierte thermische und mechanische Modelle der Straßenkonstruktion mit integriertem Kollektorsystem erstellt. Nachfolgend werden die Simulationsergebnisse der thermischen Beanspruchungen aus dem Projekt mit der in dieser Arbeit verwendeteten Methodik der Mittelwertfunktionen analysiert und die Ergebnisse dargestellt.

Grundlegend wurden im Rahmen des Forschungsprojektes zwei Typen von Straßenkonstruktionen untersucht. Die Referenzvariante bildet ein konventioneller Straßenaufbau mit der Bauweise Asphalttragschicht auf Frostschutzschicht nach den RStO 12 (2012, Tafel 1). Die Vergleichsvariante bildet der Konstruktionsaufbau mit integriertem Rohrkollektor innerhalb der Asphaltbinderschicht. Der Rohrkollektor besteht aus einem bifilar verlegten Rohr mit einem Außendurchmesser von 2,54 cm. Das Material ist ein Verbundwerkstoff aus Kunststoff, Aluminium und einer weiteren Lage Kunststoff. 
In Abbildung 7.1 ist ein schematischer Aufbau der Straßenkonstruktion mit integriertem Kollektor dargestellt. Die aufgeführten Varianten wurden als Pilotanlage auf dem Demonstrations-, Untersuchungs- und Referenzareal der Bundesanstalt für Straßenwesen (duraBASt) errichtet. Im Projekt konnte weiterhin eine Validierung der numerischen Simulationen anhand realer Messdaten durchgeführt werden.

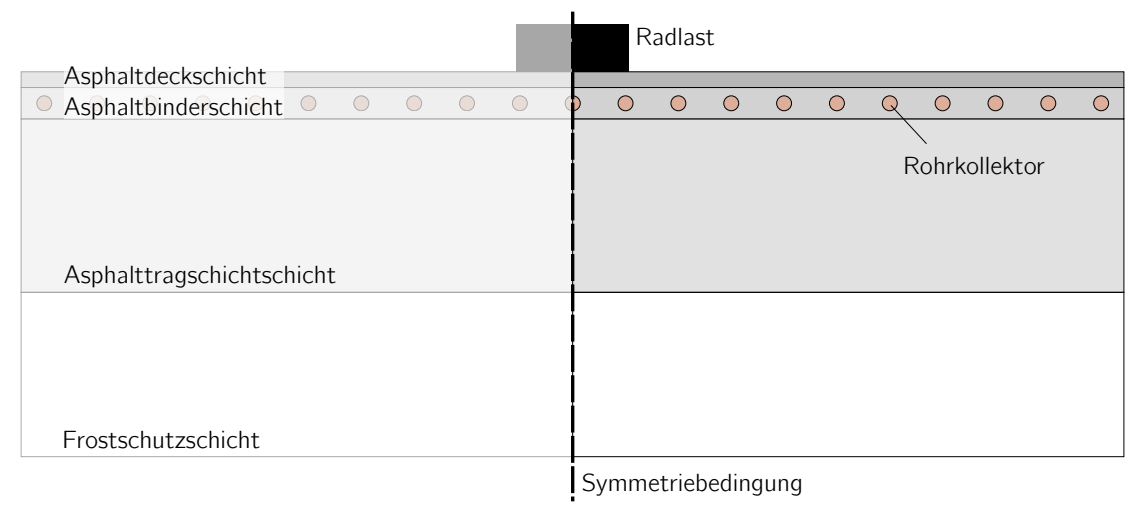

Abbildung 7.1

Schematische Darstellung des Finite Elemente Modells zur Berechnung der mechanischen Beanspruchungen

Die Berechnung der mechanischen Beanspruchungen innerhalb der beiden Straßenbefestigungen wurde mittels FE-Simulationen durchgeführt. Die Netzstruktur der zwei Modelle ist in Abbildung 7.2 dargestellt. Bedingt durch die Rohre ist eine Anwendung eines rotationssymetrischen Modells nicht möglich, daher wurde ein 3D-Modell erstellt. Die Symmetriebedingungen wurden zur Steigerung der Berechnungsgeschwindigkeit berücksichtigt. Der Eintrag der Belastungen aus dem Verkehr erfolgt mittels einer Kreislast (Halbkreis) am linken oberen Rand. Die Netzstruktur unterhalb der Last ist stark verfeinert. Aufgrund der Belastungen sind in diesem Bereich hohe Spannungsgradienten vorhanden. Eine feine Netzstruktur ermöglicht eine möglichst realistische Simulation der mechanischen Beanspruchungen. Zum Rand hin können die Elemente aufgrund abnehmender Beanspruchungen vergrößert werden. Die Integration des Rohrkollektors führt dazu, dass im Bereich um die Rohre keine Rechteckelemente verwendet werden konnten. Die Elemente in der Bettungsschicht wurden als prismatische Elemente definiert. Aus Vorstudien konnte abgeleitet werden, dass an der Ober- und Unterseite der Rohre sehr hohe Spannungsgradienten zwischen dem Rohr und den umliegenden Asphalten entstehen. Diese wurden im finalen Modell berücksichtigt, indem die Elementgröße in diesem Bereich erheblich verringert wurde. Aus Gründen der Elementanzahl des Gesamtmodells und der damit verbundenden Berechnungsgeschwindigkeit wurde die Anzahl der Rohre auf vier Stück reduziert. Weiter entfernte Rohre haben kaum Einfluss auf die mechanischen Beanspruchungen im Bereich der Lasteinleitungsfläche.

Die thermischen Zustände innerhalb der Straßenkonstruktion haben einen direkten Einfluss auf die Steifigkeit der Asphalte und somit auf das mechanische Verhalten der Straßenkonstruktion. Zu diesem Zweck wurden für beide Varianten detaillierte thermische Simulationen 


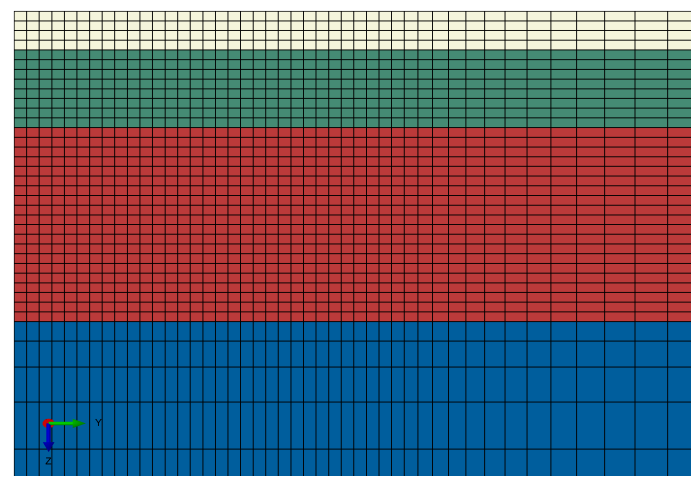

(a) Referenzvariante

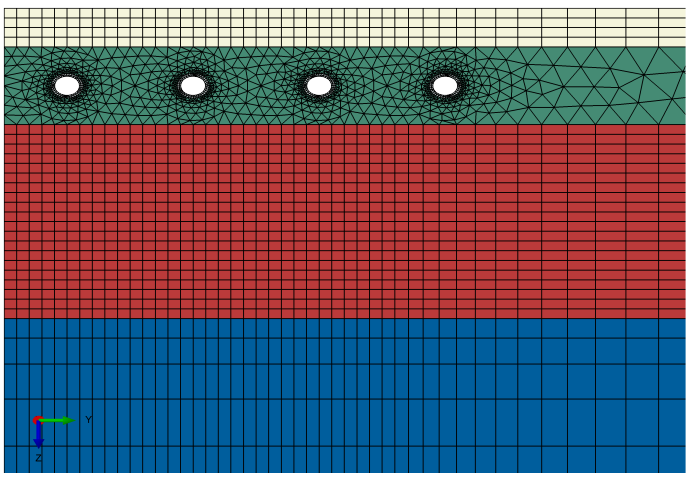

(b) Variante mit Rohrkollektor

Abbildung 7.2

\section{Darstellung der Netzstruktur der ausgewählten mechanischen Modelle}

durchgeführt. Die Eingangsparameter für die Untersuchungen bilden die gemessenen thermophysikalischen Materialparameter der Asphalte sowie fiktive, übliche Materialparameter der Frostschutzschicht und des Untergrundes. Die lokalen Klimaparameter für das Stadtgebiet Dresden wurden als Eingangsdaten für die numerischen Simulationen verwendet. Die Daten entstammen der Klimaprojektion REMO-UBA mit dem Emissionsszenario A1B (Jacob, 2005b), das auch für die anderen bereits vorgestellten Untersuchungen verwendet wurde. Die hier dargestellten Ergebnisse beruhen auf den Simulationsergebnissen des Untersuchungszeitraums 2020 bis 2049.

Die Einbettung des Rohrkollektors ermöglicht nicht nur eine Auskopplung von thermischer Energie im Sommer, sondern auch eine aktive Erwärmung der Straßenkonstruktion im Winter. Für die Variante mit integriertem Rohrkollektor wurde das Szenario der Frostfreihaltung im Winter sowie der Kühlung im Sommer mit einer Vorlauftemperatur eines Kühlkreislaufes von $30^{\circ} \mathrm{C}$ gewählt. Dieses verwendete Szenario koppelt mehrere Nutzungskonzepte und hat somit das größte wirtschaftliche Potenzial.

In Abbildung 7.3 sind die Ergebnisse der Untersuchungen getrennt nach den Asphaltschichten vergleichend dargestellt. Die Höhen der Formen entsprechen den über die Tiefe gemittelten Temperaturen je Schicht. Die Häufigkeit des Auftretens einer spezifischen Temperatur wird über die zugeordnete Breite der Form dargestellt. Innerhalb der Form ist mit einer gestrichelten Linie der Mittelwert über den gesamten Untersuchungszeitraum gekennzeichnet. Die fein gestrichelten Linien entsprechen dem 0,25-Quantil und dem 0,75-Quantil. Vergleicht man die auftretenden Temperaturen in der Asphaltdeckschicht so ist ersichtlich, dass die Referenzvariante ohne Kollektor ein größeres Temperaturspektrum aufweist. Im Winter wurden Oberflächentemperaturen bis zu $-25^{\circ} \mathrm{C}$ und im Sommer bis zu $65^{\circ} \mathrm{C}$ simuliert. Die Verwendung des Kollektors führt dazu, dass niedrige Temperaturen durch die aktive Erwärmung der Bettungsschicht hin zu wärmeren Temperaturen verschoben werden. Der Einsatz des Kollektors im Winter wurde für eine Eis- und Schneefreihaltung konzipiert. Die 
zulässige Oberflächentemperatur wurde in der Simulation auf Minimum von $5{ }^{\circ} \mathrm{C}$ begrenzt. Dies bewirkt, dass die Temperaturen in der Straßenbefestigung unterhalb der Aktivierungstemperatur des Kollektors signifikant seltener auftretenden. Dies ist in der Darstellung als deutlich verbreiterter Bereich in Abbildung 7.3b ersichtlich. Die extrem hohen Temperaturen im Sommer konnten ebenfalls durch eine aktive Kühlung der Bettungsschicht erheblich reduziert werden. Je tiefer eine Schicht liegt, desto geringer ist auch die Temperaturspanne. Die Integration eines Rohrkollektors beeinflusst damit nicht nur die oberflächennahen, sondern auch die tiefer liegenden Asphaltschichten.

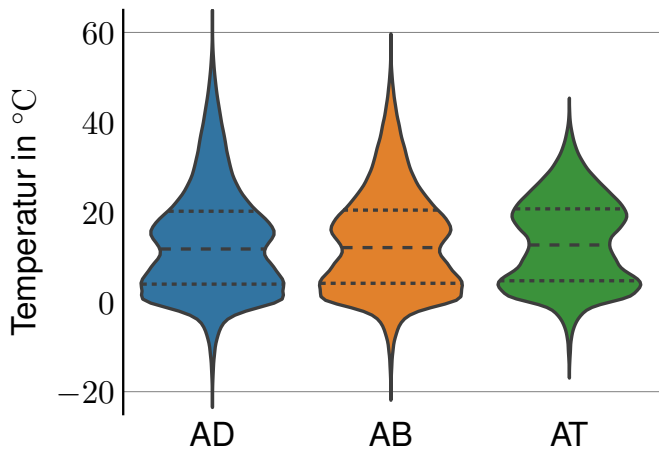

(a) Referenzvariante

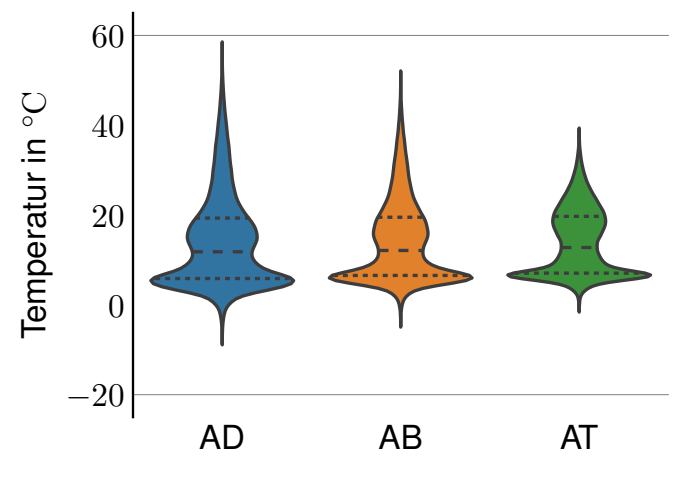

(b) Variante mit Rohrkollektor

Abbildung 7.3

Verteilung der Temperaturen innerhalb der Asphaltschichten der Straßenkonstruktionen für den Simulationszeitraum 2011 bis 2040

Für die Berechnung der thermischen Zustände innerhalb der Straßenkonstruktionen wurde das thermische Modell im Rahmen des Projektes erweitert (siehe Abschnitt 4.2), um die Berücksichtigung einer Kühlung und Heizung zu ermöglichen. Anhand der Simulationsergebnisse wurden einmal die Häufigkeitsverteilungen der normierten charakteristischen Temperaturprofilen (ncTs) nach Kayser (2007) und die konkreten Verläufe der Mittelwertfunktionen bestimmt (Abbildung 7.4).

Für die Ermittlung des Ermüdungsstatus werden die im Abschnitt 3.5 dargestellten Ganglinien des Verkehrs berücksichtigt. Dies erfordert, dass die Häufigkeitsverteilung des Auftretens der Cluster bzw. Temperaturprofile in Abhängigkeit der Tageszeit ermittelt werden (Abbildung 7.5). Der Vergleich zeigt erhebliche Unterschiede zwischen den Auswertungsalgorithmen. Die Verteilung auf Basis der ncTs hat einzelne Bereiche mit zum Teil sehr hohen Häufigkeiten des Auftretens eines Zustandes. Weiterhin ist die im Verfahren verwendete Einteilung nach der Tageszeit gut sichtbar. Die Clusteranalyse der Ergebnisse der thermischen Simulation ergibt ein deutlich differenzierteres Ergebnis. Die Mehrheit der Temperaturzustände (die ersten 80 Cluster) kommen im Laufe des gesamten Tages vor, wobei die Häufigkeit des Auftretens innerhalb der Periode schwankt. Tendenziell kommen diese Temperaturprofile 


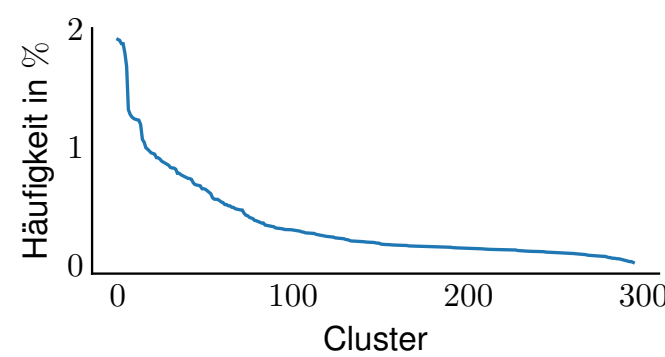

(a) Mittelwertfunktionen

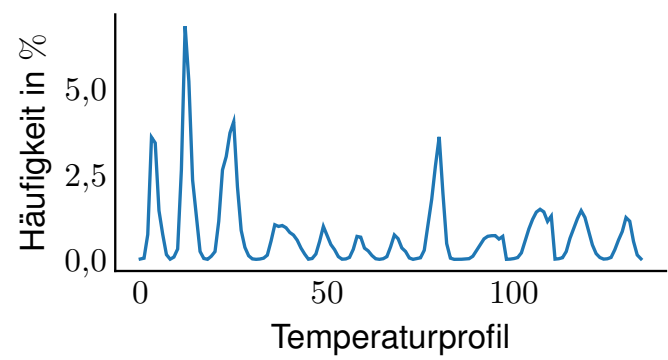

(b) Normierte charakteristische Temperaturprofile

Abbildung 7.4

\section{Häufigkeitsverteilung der Cluster bzw. Temperaturprofile der Variante mit Rohrkollektor}

vermehrt in den Nachtstunden vor. Mit Abnahme der Häufigkeit eines Clusters (höhere Nummern) nimmt gleichzeitig auch die Spannweite des Zeitraums des Auftretens ab.

Die Anwendung des Clusterverfahrens zur Bestimmung der Mittelwertfunktionen ergibt die in Abbildung 7.6 dargestellten repräsentativen Temperaturprofile. Der direkte Vergleich der beiden konstruktiven Varianten zeigt, dass die Spannweite der Temperaturen innerhalb der Straßenkonstruktion durch den Einsatz des Kollektors deutlich gemindert werden konnte. Temperaturen im negativen Spektrum kommen auch nach Anwendung des Clusterverfahrens nicht vor. Die Reduktion der hohen Temperaturen im Bereich der Aspahltbinder- und Asphalttragschicht konnten auch in den Ergebnissen der Analyse nachgewiesen werden. Anhand der repräsentativen Verläufe ist ersichtlich, dass die Anwendung des Verfahrens zur Bestimmung der Mittelwertfunktionen zu einer erheblichen Steigerung der Genauigkeit führt. Reale thermische Zustände in Straßenkonstruktionen werden damit umfänglich berücksichtigt.

Die Abweichung zwischen den simulierten Daten und den zugehörigen Temperaturprofilen ist ein Indikator für die Güte des verwendeten statistischen Verfahrens. In Abbildung 7.7 sind die Temperaturabweichungen dargestellt. Die weiße Hintergrundfarbe bedeutet, dass die berechnete Abweichung des Temperaturprofils kleiner als der Minimalwert der Legende ist. Eine dunkelrote Farbe zeigt hingegen an, dass der obere Wert der Skala überschritten ist, also eine deutlich höhere Abweichung als angegeben besteht. Der direkte Vergleich zwischen den statistischen Verfahren ergibt sowohl für die Referenzvariante als auch die dargestellte Variante mit Rohrkollektor eine erheblich geringere Differenz bei der Verwendung der Mittelwertfunktionen. In einer Vielzahl an Clustern liegt die Temperaturabweichung unterhalb des angezeigten Minimalwertes von $5,50^{\circ} \mathrm{C}$. Das Verfahren der Mittelwertfunktionen ergibt nur bei einer sehr geringen Anzahl an repräsentativen Temperaturprofilen eine erhöhte Abweichung. Diese kommen vorwiegend bei Clustern vor, welche selten bis sehr selten auftreten. Durch eine Erhöhung der Clusteranzahl wäre hier eine weitere Verringerung der Abweichung möglich. Dies führt aber auch zu einer Erhöhung der Zustände im Dimen- 


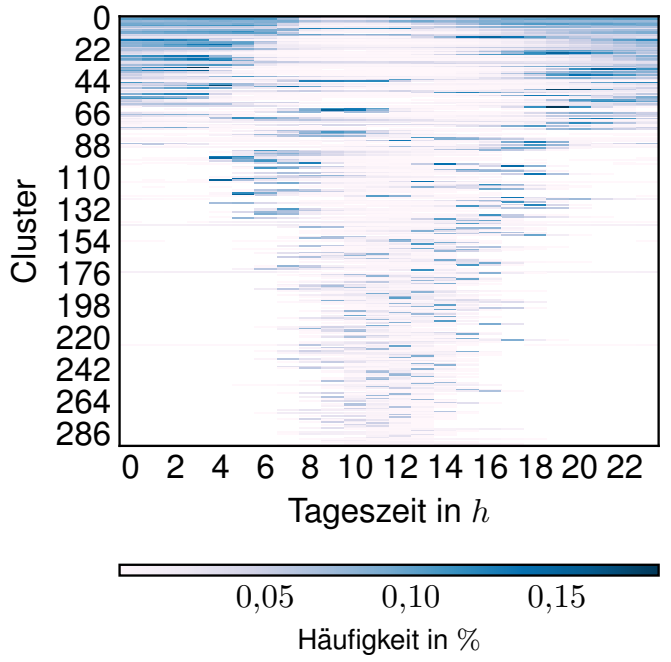

(a) Mittelwertfunktionen

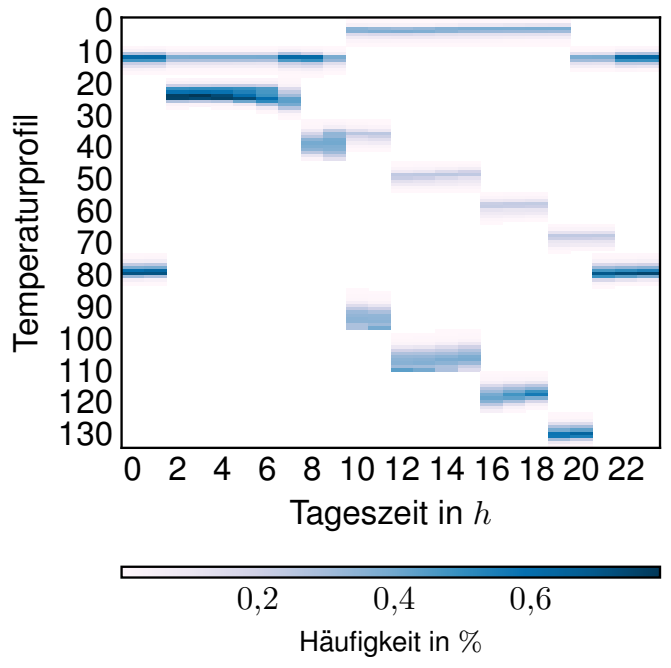

(b) Normierte charakteristische Temperaturprofile

Abbildung 7.5

Häufigkeitsverteilung der Cluster bzw. Temperaturprofile in Abhängigkeit der Tageszeit; Variante mit Rohrkollektor

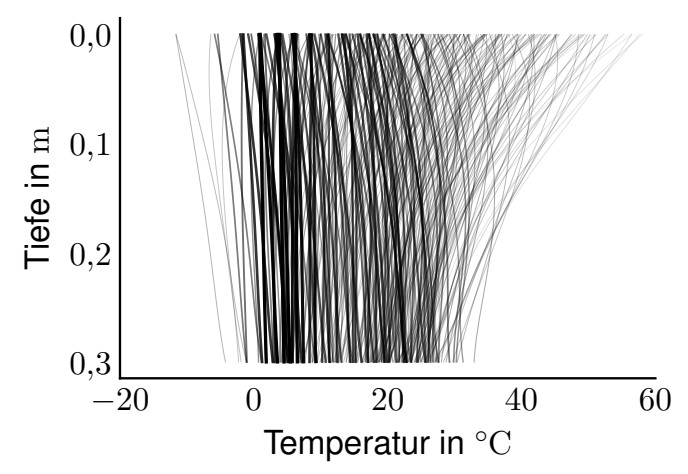

(a) Referenzvariante

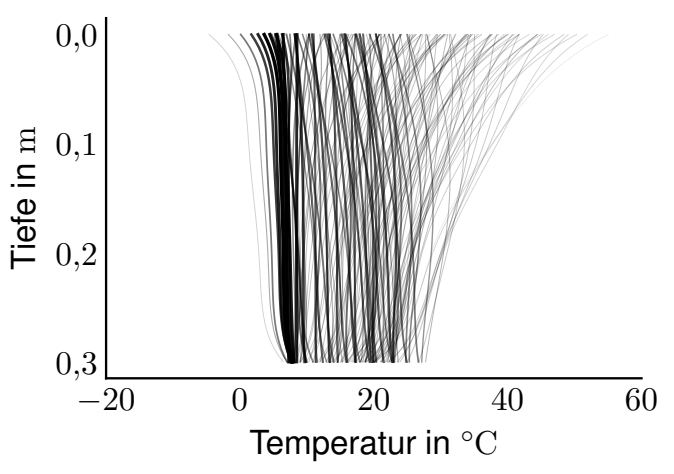

(b) Variante mit Rohrkollektor

Abbildung 7.6

Verläufe der Clusterzentren 
sionierungsverfahren, welche durch mechanische Berechnungen bewertet werden müssen.

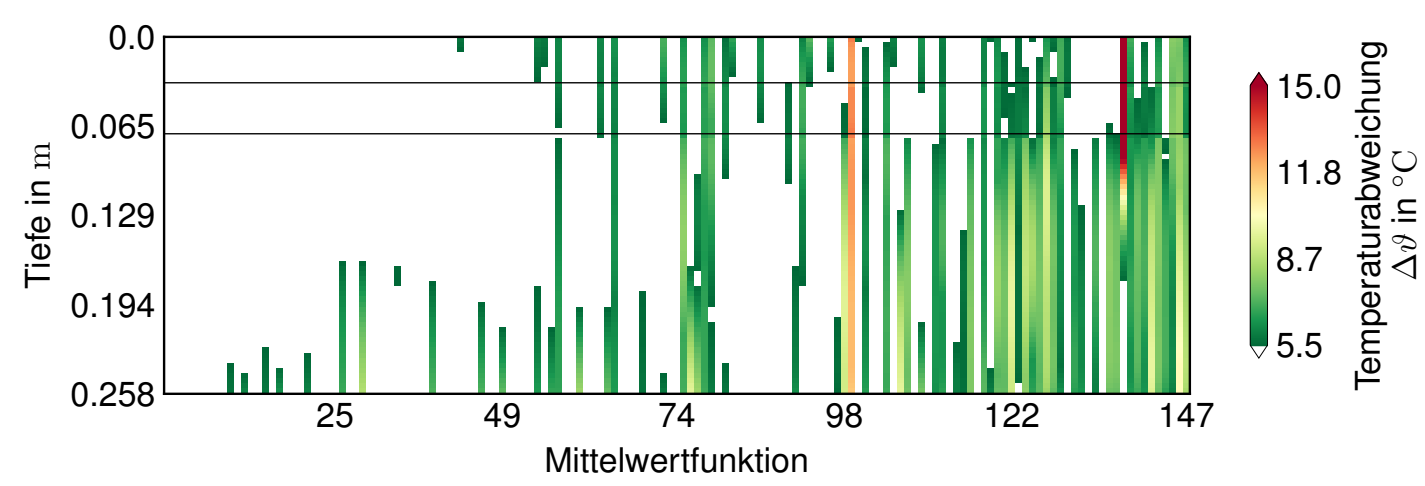

(a) Mittelwertfunktionen

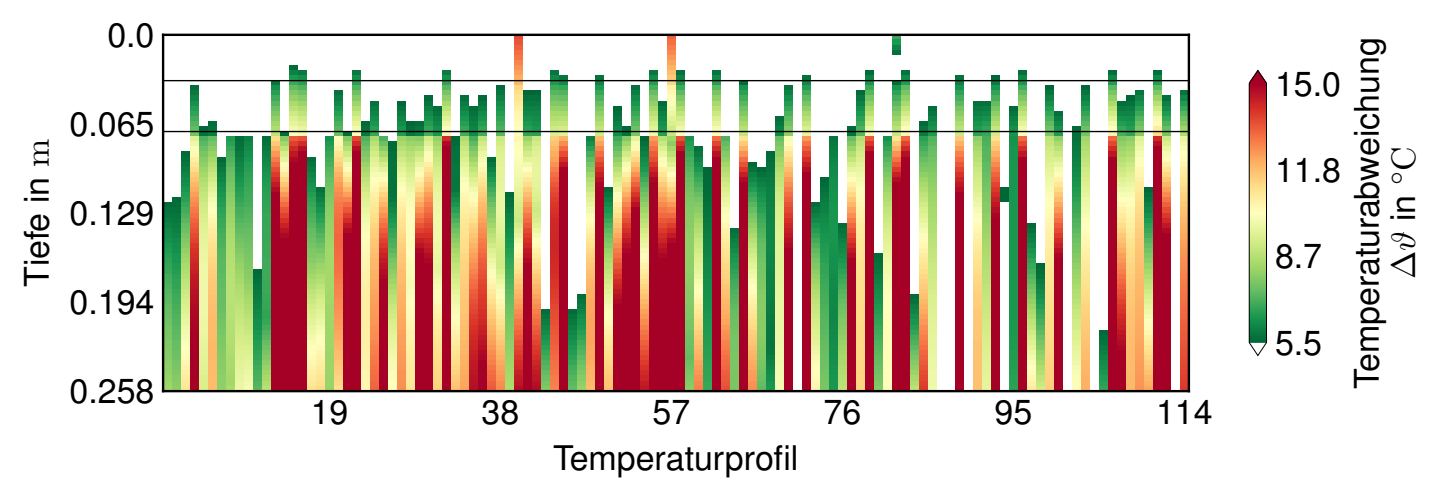

(b) Normierte charakteristische Temperaturprofile (ncTs)

Abbildung 7.7

Abweichung zwischen den repräsentativen Temperaturprofilen und den zugehörigen simulierten Temperaturbedingungen innerhalb der Straßenkonstruktion; Variante mit Rohrkollektor

Die Verwendung der ncTs ergibt für den Bereich der Asphaltdeckschicht sehr gute Übereinstimmungen zwischen dem statistischen Mittelwert und den zugehörigen Temperaturbedingungen. In den tieferen Schichten steigen die Temperaturabweichungen jedoch erheblich an und sind somit nicht mehr repräsentativ für die tatsächlichen Temperaturzustände. In der Mehrzahl der bestimmten ncTs steigen die Abweichungen auf einen Wert größer als $10,00^{\circ} \mathrm{C}$. In einer signifikanten Anzahl konnten Abweichungen von mehr als $15,00^{\circ} \mathrm{C}$ festgestellt werden. Dies wirkt sich auf die Ergebnisse der Dimensionierungsberechnungen aus. Auf einen Vergleich zwischen den Verfahren wird an dieser Stelle verzichtet. Die Ergebnisse auf Basis der ncTs können dem Forschungsbericht entnommen werden (Clauß u. a., 2019, S. $80 \mathrm{ff}$.$) .$ 


\section{Fallbeispiele}

Mittels der repräsentativen Temperaturprofile auf Basis der Mittelwertfunktionen wurden Berechnungen zur Bestimmung der mechanischen Beanspruchungen mit der Software ABAQUS durchgeführt. Die Ergebnisse wurden anschließend aufbereitet und der Ermüdungsstatus nach dem im Kapitel 3.2 beschriebenen Verfahren berechnet. Die Parameter wurden zum Vergleich identisch zu den Annahmen im Forschungsprojekt (Clauß u. a., 2019, Tabelle 7.3, S.81) festgelegt. Um die Charakteristiken der Rohrkollektoren in den untersuchten Straßenkonstruktionen zu berücksichtigen, wurde weiterhin das erweiterte Verfahren unter Nutzung der Hauptdehnungen genutzt (Abschnitt 3.4).

Die Verteilung der potenziellen Nachweispunkte der untersuchten Varianten ist in Abbildung 7.8a und Abbildung 7.8b dargestellt. Die graue Fläche auf der Oberseite der Fahrbahn gibt die Lasteinleitungsfläche an. In dieser Variante wurde mit einer quadratischen Fläche äquivalent zu einem Kreis mit Radius 150 mm gerechnet.

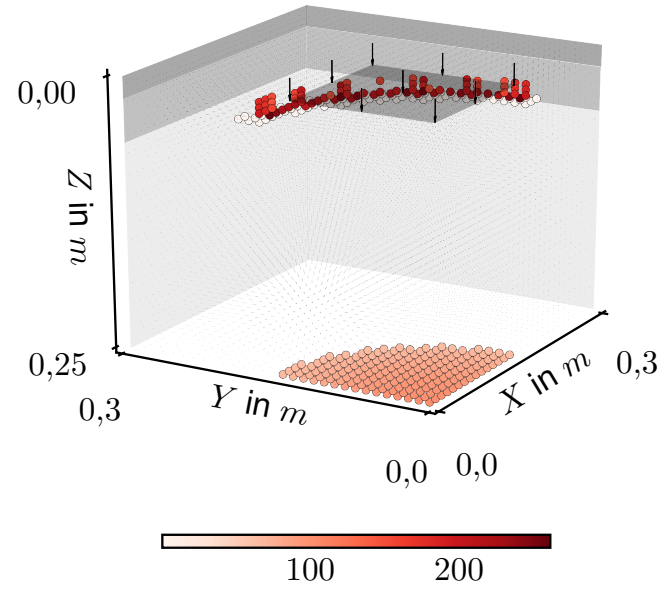

rel. Ermüdungsstatus in \%

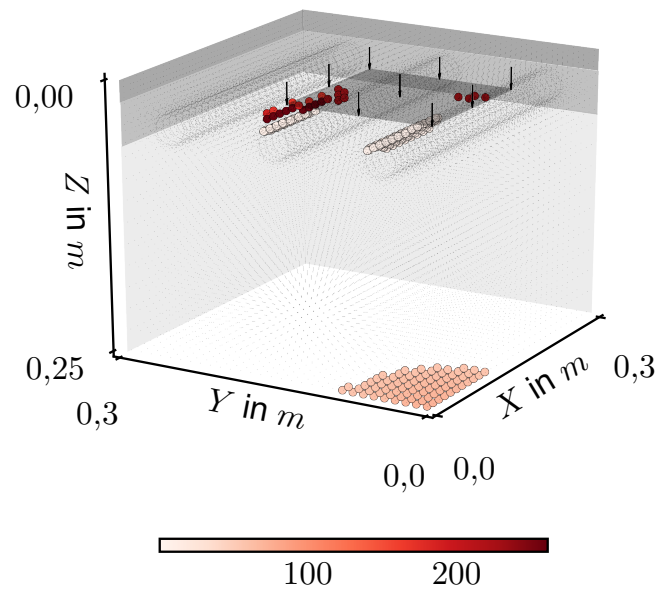

rel. Ermüdungsstatus in \%

(b) mit Kollektorsystem

Abbildung 7.8

Ermüdungsstatus in Abhängigkeit der Lage innerhalb der Straßenkonstruktion; Vergleich zwischen Referenzvariante und Variante mit integriertem Rohrkollektorsystem

Die Materialparameterkombinationen führen zu einem deutlich erhöhten Schadensrisiko in der Asphaltdeckschicht der Referenzvariante (vgl. Abbildung 7.8a). Entlang des äußeren Randes der Lasteinleitungsfläche wurden signifikant höhere Schädigungen ermittelt. Die durch die Belastung bedingten Hauptdehnungen, welche alle Komponenten des Verzerrungstensors berücksichtigen, führen infolge der größeren, absoluten Dehnungen zu einer rechnerisch früheren Entstehung von Ermüdungsrissen in der Asphaltdeckschicht. Es gilt zu beachten, dass die Schichtdicke auf ein Minimum reduziert wurde, um möglichst viel thermische Energie aus dem System auszukoppeln. Dies geht mit einer Verkürzung der Nutzbarkeit 
der Asphaltdeckschicht einher. In der Asphaltbinderschicht konnten keine nennenswerte Schädigungen detektiert werden. An der Unterseite der Asphalttragschicht entstehen starke horizontale Dehnungen, welche zu Ermüdungsrissen führen können.

Die Einbettung des Rohrsystems hat zur Folge, dass eine Beeinflussung der Lastverteilung bis in die Tiefe der Straßenkonstruktion erfolgt (Abbildung 7.8b). Oberhalb der Rohrleitungen entstehen konzentrierte Spannungsspitzen. Der Rohrkollektor wird mit einem Medium gefüllt und mit einem Innendruck von 1,50 bar betrieben. Weiterhin ist die resultierende Gesamtverformung des Rohres unter Last geringer als die Verformung des Asphaltes bei hohen Temperaturen. Der Asphalt verformt sich entlang des Rohres. Diese führen zu erhöhten Dehnungen in der Asphaltbinder- und Asphaltdeckschicht, was mit einer deutlichen Zunahme des Risikos auf Ermüdungsrissbildung einhergeht. Die Berechnungen ergeben, dass die Nutzungszeiträume der Schichten um den Faktor zwei bis drei sinken. Die Auskopplung der thermischen Energie durch den Kollektor führt zu einer Absenkung von höheren Temperaturen in der Tiefe der Straßenkonstruktion. Dies bewirkt, dass die berechnete Schädigung an der Unterseite der Asphalttragschicht abnimmt. Das Risiko für die Entstehung von Ermüdungsrissen sinkt in diesem Bereich.

In Abbildung 7.9 sind die Ergebnisse der Untersuchungen vergleichend dargestellt. Dabei wurden die Eingangsparameter für die Durchführung der Dimensionierungsrechnungen nicht verändert, also sind die Verkehrslasten und die Sicherheitsbeiwerte zwischen den Varianten als konstant definiert. Die thermischen Zustände innerhalb der Straßenkonstruktionen wurden jeweils nach dem Verfahren der Mittelwertfunktionen und der ncTs analysiert und für die Berechnung der mechanischen Beanspruchungen verwendet. Für den Vergleich der Einzelrechnungen wurde die Referenzvariante auf Basis der Mittelwertfunktionen gewählt und die maximale Schädigung je Asphaltschicht bestimmt. Diese Werte bilden den Basiswert für die nachfolgenden Analysen.

Im Bereich der Asphaltdeckschicht sinkt die relative Änderung der Nutzungsdauer bei der Referenzvariante unter Verwendung der ncTs um ca. 49\%. Die Integration des Rohrkollektors hat eine deutlich negative Beeinflussung auf die Nutzungsdauer der Schicht. Der Vergleich der Asphaltbinderschicht zwischen den Varianten mit und ohne Rohrkollektor ist kaum möglich. Die Einbettung der Rohre bewirkt eine deutliche Zunahme von lokalen Spannungsspitzen mit der Folge, dass Risse kurz nach Beginn der Verkehrsfreigabe entstehen. Der Einsatz eines Rohrkollektors hat rechnerisch ein deutliches Potenzial sowohl bei der Verwendung der ncTs (siehe Differenz zwischen Referenzvariante und Variante mit Rohrkollektor) als auch der Mittelwertfunktionen. Unter der Berücksichtigung der Tagesganglinien des Verkehrs ist eine Verlängerung der Nutzungszeiten für die Asphalttragschicht um 37,70 \% möglich, wohingegen die Berechnungsergebnisse auf Basis der ncTs eine deutlich geminderte Veränderung von 7,50 \% (Differenz zwischen Variante mit Rohrkollektor und der Referenzvariante auf Basis der ncTs) ergeben.

Sowohl die Berechnungen innerhalb des Forschungsprojektes als auch die ergänzenden Untersuchungen in dieser Arbeit zeigen, dass ein erhebliches Potenzial für die Verwendung von Kollektorsystemen zum Thermomanagement von Straßenkonstruktionen besteht. Die 


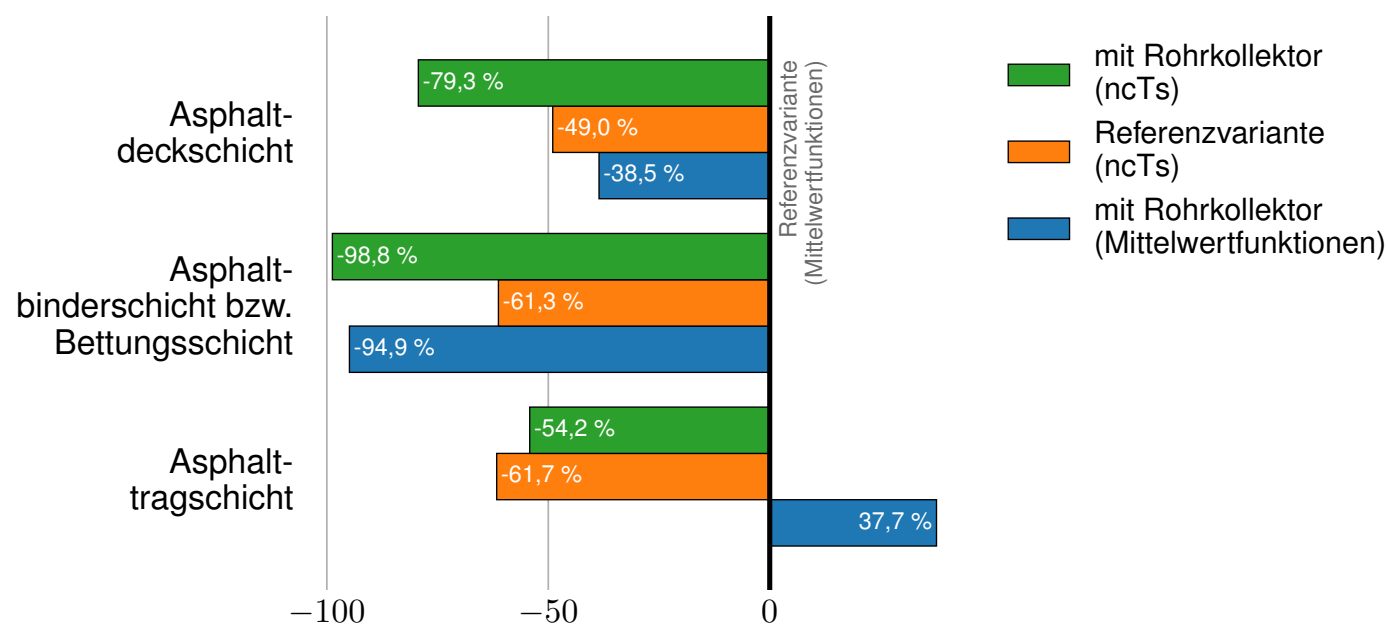

rel. Änderung der Dauerhaftigkeit in \%

Abbildung 7.9

Vergleich der relativen Änderungen der Nutzungsdauer zwischen der Referenzvariante und einem Straßenaufbau mit integriertem Kollektorsystem jeweils auf Basis der Mittelwertfunktionen oder normierten charakteristischen Temperaturprofilen; Referenzwert bildet der maximale Ermüdungsstatus der Referenzvariante auf Basis der Mittelwertfunktionen

Betrachtungen in dieser Arbeit konnten zeigen, dass die Verwendung der ncTs zu starken Abweichungen zwischen realen Temperaturbedingungen und den repräsentativen Temperaturen bestehen. Ein aktives Temperaturmanagement beeinflusst die realen Temperaturbedingungen in der gesamten Straßenkonstruktion erheblich. Das entwickelte Verfahren der Mittelwertfunktionen ermöglicht eine Minimierung der notwendigen Berechnungsdurchläufe unter einer gleichzeitig hohen Güte der statistischen Betrachtungen. Aus diesem Grund sollte das Verfahren der Mittelwertfunktionen für zukünftige Betrachtungen verwendet werden.

\subsection{Hitzeperiode}

In den Jahren 2018 und 2019 stellte das Wetter in vielen Regionen Deutschlands die Bevölkerung und Natur vor enorme Herausforderungen (Kaspar und Friedrich, 2020). In Europa herrschten extrem hohen Temperaturen und lang anhaltende warme Perioden. Es hat sich gezeigt, dass diese Wetterereignisse einen erheblichen Einfluss auf die Verkehrsinfrastruktur haben.

Zur Analyse des Einflusses wurden für die Region Dresden (Wetterstation Dresden Klotzsche) Messdaten der Wetterbedingungen ab Sommer 2012 heruntergeladen und aufbereitet. Die Daten liegen in einer zeitlichen Auflösung von 15 min vor. Auf Basis der verfügbaren Wetterreihen wurden thermische Simulationen vorgenommen. Die Randbedingungen der 
Simulationen, wie die thermophysikalischen Materialkennwerte oder der Reflexionsbeiwert, wurden analog zu den Angaben in Abschnitt 4.3 gewählt.

In Abbildung 7.10 sind die simulierten Oberflächentemperaturen der Straßenkonstruktion dargestellt. Die Abbildung zeigt, dass wie zu erwarten eine ausgeprägte Temperaturdifferenz im Betrachtungszeitraum simuliert werden konnte. Weiterhin konnten deutlich unterschiedliche Oberflächentemperaturen zwischen Winter und Sommer ermittelt werden.

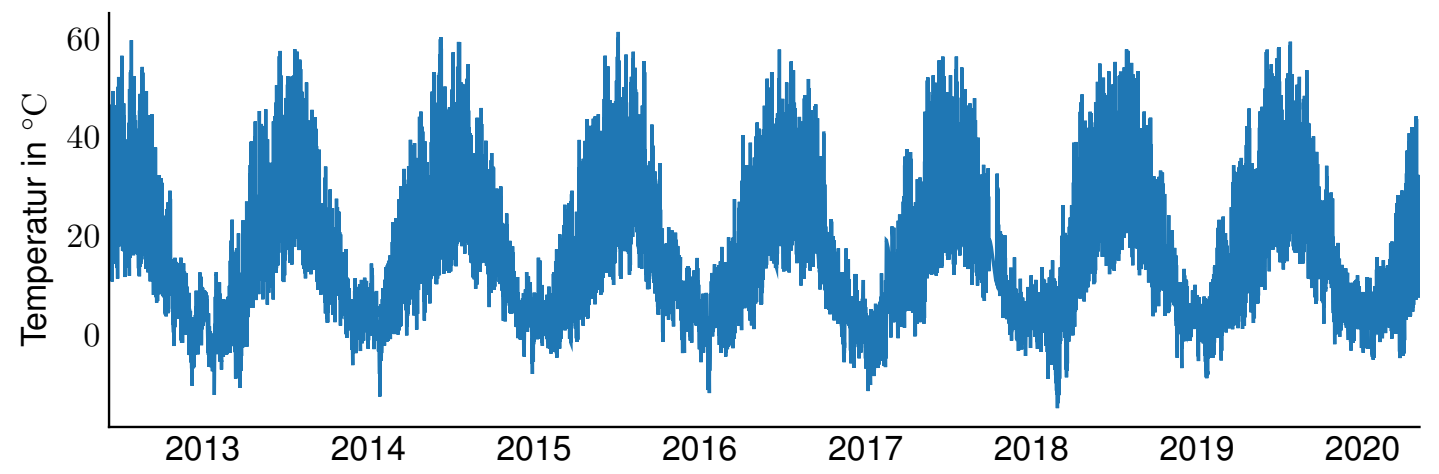

Abbildung 7.10

Oberflächentemperaturen der Simulationen auf Basis von Wetterdaten (Station Dresden Klotzsche)

Der relativ kurze Zeitraum ermöglicht kaum die Ermittelung von Langzeittrends, jedoch konnten innerhalb dieser kurzen Periode deutliche Unterschiede bei den Beobachtungen festgestellt werden. Dazu wurde die mittlere Temperatur je Monat für den Zeitraum 2013 bis 2018 ermittelt. Ein Vergleich der Monatsmittelwerte eines Betrachtungsjahres mit den langjährigen Monatsmittelwerten zeigt auftretende Temperaturanomalien (Abbildung 7.11). Diese Betrachtung zeigt, dass z. B. im Juni 2019 eine über $6{ }^{\circ} \mathrm{C}$ höhere Monatstemperatur auf der Oberfläche der Asphaltbefestigung simuliert wurde. Die verheerenden klimatischen Bedingungen im Jahr 2018 und 2019 führten dazu, dass in 15 von 24 Monaten eine positive Temperaturanomalie festgestellt werden konnte. Die Messdaten für 2020 ergeben, dass die Temperaturen der ersten vier Monate des Jahres ebenfalls über dem langfristigen Trendwert lagen.

Für die Simulationsergebnisse wurde eine Ermittelung der statistisch relevanten Temperaturbedingungen mittels des Clusterverfahrens zur Bestimmung der Mittelwertfunktionen durchgeführt. Die Häufigkeit des Auftretens der einzelnen Cluster in Abhängigkeit von der Tageszeit sind in Abbildung 7.12 dargestellt. Die berechneten Mittelwertfunktionen treten nicht konstant über den Tag verteilt auf, sondern es wurde eine erhebliche Abhängigkeit von der Tageszeit festgestellt, die bei den Prognoserechnungen zu berücksichtigen ist.

Die ermittelten Verläufe der für die Nachweise verwendeten Temperaturprofile sind in Abbildung 7.13 dargestellt. Alle Profile verlaufen innerhalb der Spannweite der auftretenenden 


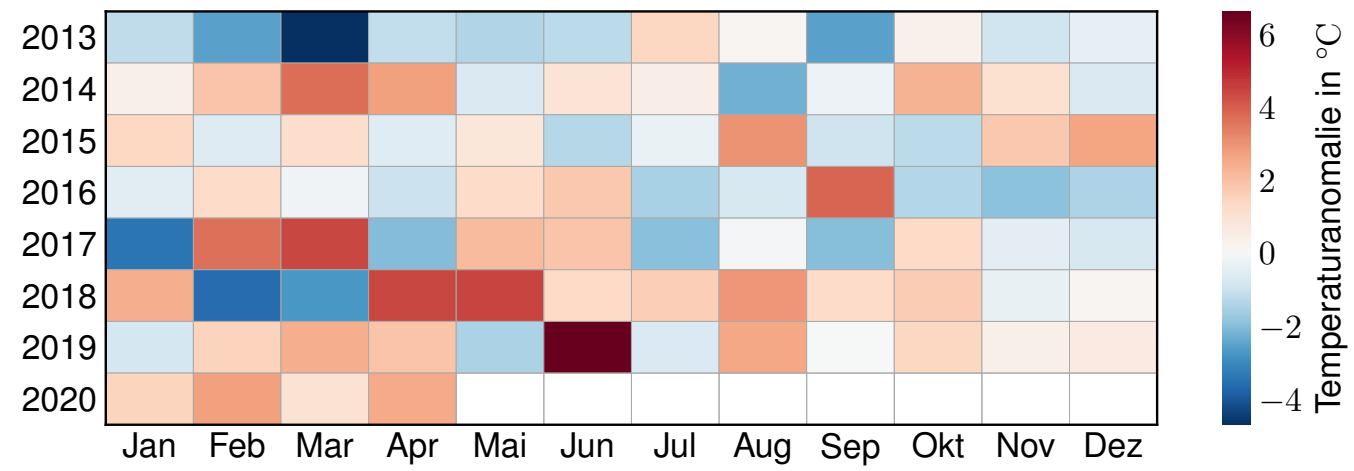

Abbildung 7.11

Anomalie der simulierten Oberflächentemperaturen, Referenzzeitraum 2013 bis 2017

Temperaturen der Straßenbefestigung. Auf Basis der Verläufe wurden Berechnungen zur Bestimmung der mechanischen Beanspruchungen in der Straßenbefestigung vorgenommen. Bedingt durch die schnelle Simulationszeit erfolgte die Berechnung der Beanspruchungen auf Basis der Mehrschichtentheorie. Es wurde jedoch nicht nur mit einem einzelnen Nachweispunkt, sondern mit einem dichten Netz von Punkten gerechnet. Dies ermöglicht es, analog zu FEM-Rechnungen, eine Bewertung der Anfälligkeit bzw. des Risikos gegenüber einer Bildung von Ermüdungsrissen in der gesamten Straßenbefestigung vorzunehmen. Für die Bestimmung des Ermüdungsstatus jedes Nachweispunktes wurde für jedes Jahr eine feste Anzahl an Achsübergängen verwendet. Weiterhin wurden die Achslastkollektive mit der Tagesganglinie der Verkehrsstärken (siehe Kapitel 3.5) überlagert. Die Berechnung der Nutzungsdauer erfolgte unter Verwendung der Hauptdehnungen.

Die Prognoserechnungen ergeben, dass ein Zusammenhang zwischen den klimatischen Bedingungen und den Schädigungen der Straßenkonstruktion besteht. Für die Periode von 2013 bis 2017 konnten im Bereich der Asphalttragschicht eher konstante Schädigungsraten ermittelt werden. Seit dem Jahr 2017 konnte eine erheblich erhöhte Schädigungsrate festgestellt werden. Die klimatischen Bedingungen haben einen etwas differenzierten Einfluss auf die Nutzungszeiten der Asphaltdeckschicht. Bezogen auf den Mittelwert des Referenzzeitraumes ergaben die Simulationen, dass in der Periode 2013 bis 2015 nur geringe Unterschiede bei der Schädungsrate vorhanden waren und im Jahr 2016 ist diese signifikant abgefallen. Seit 2016 musste ein kontinuierlicher Anstieg der Schädigungsrate festgestellt werden, wobei dieser seit 2018 deutlich oberhalb der mittleren Schädigungsrate liegt. 


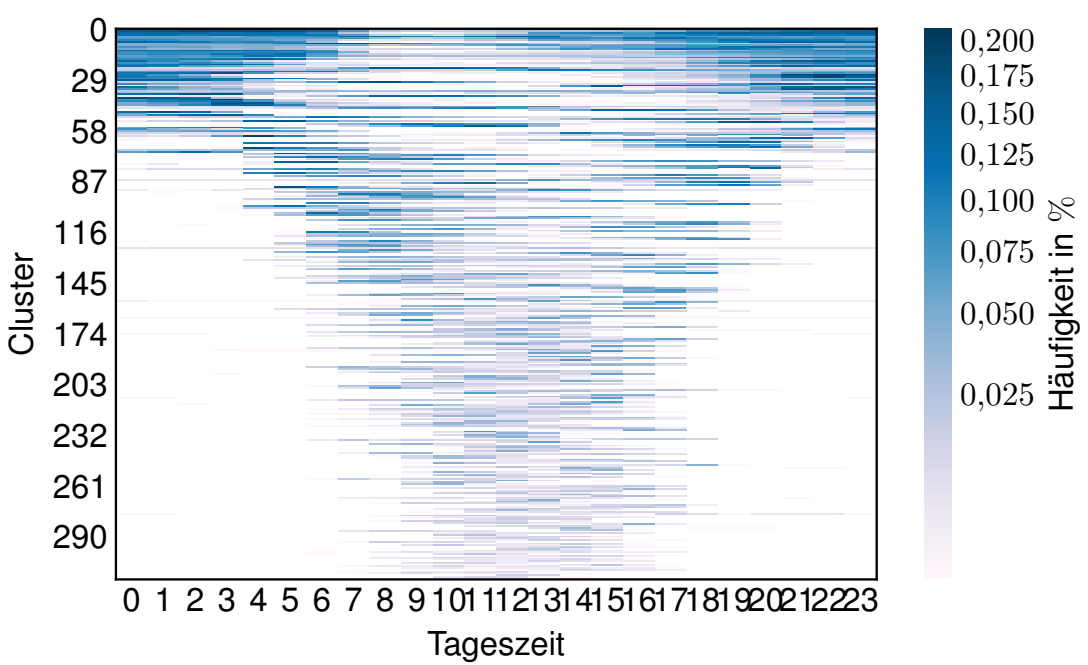

Abbildung 7.12

Häufigkeitsverteilung der Mittelwertfunktionen in Abhängigkeit der Tageszeit

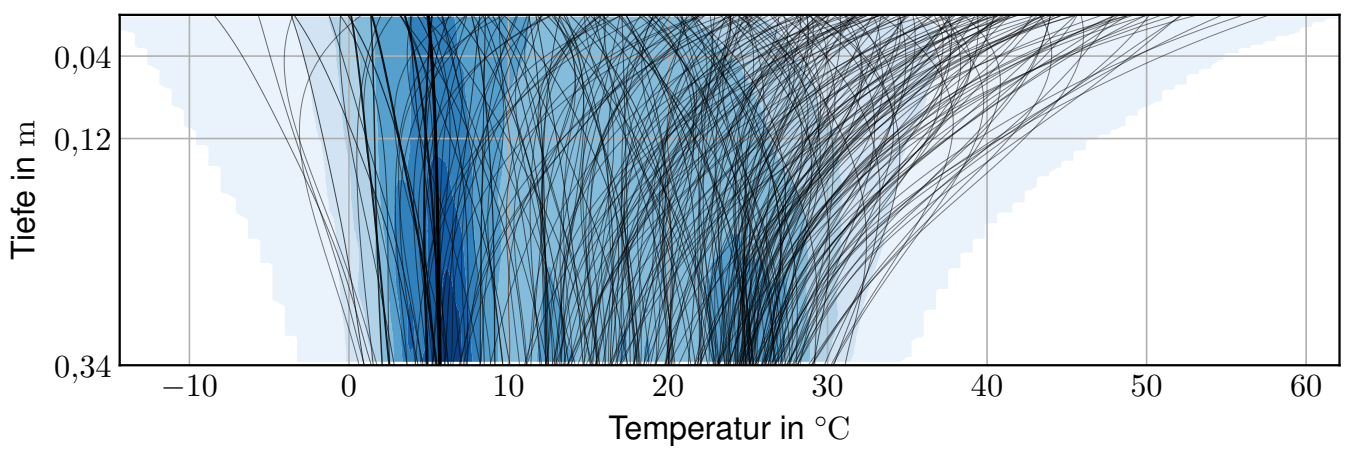

Abbildung 7.13

Verlauf der Mittelwertfunktionen der Temperaturzustände für die Simulation mit Wetterdaten Station Dresden Klotzsche 
7 Fallbeispiele

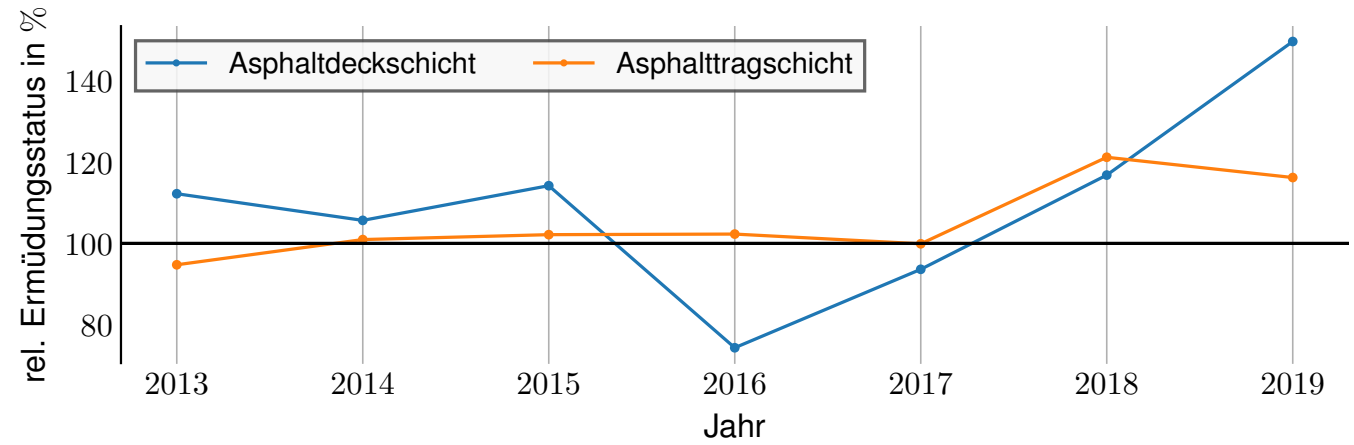

Abbildung 7.14

Relative Änderung der Nutzungszeit, Referenzzeitraum 2013 bis 2017 


\subsection{Phase Change Materials}

Da in den bisherigen Untersuchungen ein negativer Einfluss des Klimawandels auf die Dauerhaftigkeit von Asphaltstraßenkonstruktionen deutlich aufgezeigt werden konnte, soll in diesem Abschnitt eine weitere konstruktive und innovative Idee zum Management der Temperaturen innerhalb der Straßenkonstruktion vorgestellt und analysiert werden. Der Ansatz besteht darin, mit einem im Asphalt eingebetteten Speichermaterial den Anstieg (Sommer) oder den Abfall (Winter) der Temperatur in der Straßenbefestigung zu dämpfen und dadurch extreme Temperaturzustände zu reduzieren.

Als besonders vielversprechend werden dabei sogenannte Phasenwechselmaterialien oder Phase Change Materials (PCM) angesehen. Diese Materialgruppe kennzeichnet sich dadurch aus, dass innerhalb des Arbeitstemperaturbereiches ein Phasenwechsel durchlaufen wird, in dem große Wärmemengen bei kleinen Temperaturdifferenzen gespeichert werden können. Bei der Aufheizung schmilzt das PCM unter Wärmeaufnahme, bei der Abkühlung des Materials wird die gespeicherte Wärmeenergie wieder an das umliegende Asphaltmaterial abgegeben.

Da viele Stoffe mit unterschiedlichsten Schmelzpunkten als Phasenwechselmaterialien (PCM) infrage kommen, können viele Speicheranwendungen vom Kälte- bis zum Hochtemperaturwärmespeicher mit dieser Technik abgedeckt werden. Die bekanntesten kommerziellen Anwendungen sind derzeit aber noch Kühlakkus und Wärmekissen. Es gibt jedoch auch Anwendungsbereiche im sommerlichen und winterlichen Wärmeschutz von Gebäuden (Cabeza u. a., 2011; Sharma u. a., 2009). Durch Einbringen von PCM in Putze oder Gipsbauplatten erhöht sich die thermische Kapazität und verzögert im Sommer die Erhöhung der Raumtemperatur bis in die Nachtstunden. Durch eine Abkühlung der Raumluft wechselt das Phasenwechselmaterial erneut in den festen Aggregatzustand.

Dieser Effekt kann theoretisch auf Straßenkonstruktionen übertragen werden, wobei das PCM in die Struktur von Asphalten eingebettet werden könnte. Denkbar ist, dass entweder Bestandteile der Asphaltrezeptur durch PCM substituiert werden oder das eine offenporige Asphaltschicht hergestellt wird, in deren Hohlräume PCM eingebettet werden. Die PCM sollten dazu (mikro-)verkapselt sein, um mögliche Wechselwirkungen mit dem Asphalt auszuschließen. Bevor ein praktischer Einsatz der Bauweise möglich ist, müssen ausführliche Untersuchungen zur Recyclingfähigkeit des Verbundmaterials aus PCM und Asphalt durchgeführt werden.

Die Substitution von Bestandteilen im Asphalt erfordert einen sehr hohen Forschungsbedarf, da die Ersetzung von Füller- und Feinstkornanteilen in Asphalten einen erheblichen Einfluss auf das Gebrauchsverhalten hat (M. Z. Chen u. a., 2011; M. Chen, Wan und Lin, 2012; Cao u. a., 2011).

Eine Einbettung in eine offenporige Asphaltschicht hat den Vorteil, dass Konzepte aus dem konventionellen Straßenbau adaptiert werden können. Die PCM füllen die Zwischenräume, kommen jedoch durch die Mikroverkapselung nicht in direkten Kontakt mit dem Bitumen. Eine Beeinflussung der Materialkennwerte des Bindemittels ist dadurch nicht zu erwarten. 


\section{Fallbeispiele}

Im Rahmen von Voruntersuchengen wurde eine Asphaltplatte mit einem Hohlraumgehalt von $15 \mathrm{M} \%$ im Labor hergestellt. Der Zwischenraum wurde anschließend mit einem PCM komplett gefüllt. Zum Einsatz kam hier ein Salzhydrat, welches günstige Materialkennwerte aufweist. Bei der Phasenänderung von fest zu flüssig darf kein Wasser aus dem Substrat verdampfen oder Kondenswasser eingelagert werden, da sich sonst die Eigenschaften des Salzhydrates verändern. Um dies zu vermeiden, wurde die Asphaltplatte in mehreren Lagen mit Kunststofffolie verpackt. Im Kern der Platte wurden Temperatursensoren angebracht und die Kerntemperatur während der Beprobungsphase kontinuierlich aufgezeichnet.

Die Theorie besagt, dass beim Erwärmen des Asphaltes die Kerntemperatur kontinuierlich zunimmt. Beim Erreichen des Schmelzpunktes kommt es zu einer starken Abflachung des Temperaturgradienten, die Temperatur im Asphalt verändert sich, bis zum kompletten Aufschmelzen des PCM, nur geringfügig. Wenn die Speicherfähigkeit des PCM aufgebraucht ist, dann steigt erneut die Temperatur des Asphaltes weiter an. Sinkt anschließend die Umgebungstemperatur unterhalb des Schmelzpunktes, so wird der Vorgang umgekehrt. Die gespeicherte Energie wird langsam abgegeben, so dass eine Auskühlung nicht so stark erfolgt. Nach Abgabe der gesamten Energie folgt ein übliches Abkühlverhalten.

Das Verhalten wurde experimentell überprüft. Dazu wurden zwei Asphaltplatten mit einer sehr offenporigen Struktur und einer Dicke von $6 \mathrm{~cm}$ im Walzsektor-Verdichtungsgerät hergestellt. Eine Platte wurde nach Auskühlung mit einem PCM (Natriumacetat-Trihydrat) gefüllt (Abbildung 7.15a). Dazu wurde die Temperatur des Salzhydrates über dessen Schmelztemperatur von $58{ }^{\circ} \mathrm{C}$ erhöht und das PCM konnte flüssig in die Hohlräume eingefüllt werden. Das Material wurde aus Gründen der kostengünstigen Verfügbarkeit gewählt. Die Schmelztemperatur von $58^{\circ} \mathrm{C}$ ist für den Einsatz in Straßenkonstruktionen unbrauchbar, jedoch kann der positive Effekt des Einsatzes von PCM in Asphaltschichten damit demonstriert werden. Im Plattenmittelpunkt wurden drei Temperatursensoren zur Erfassung der Temperaturzustände angebracht. Die Asphaltplatten wurden in einer Temperierkammer gelagert und die Lufttemperatur alternierend zwischen $40^{\circ} \mathrm{C}$ und $75^{\circ} \mathrm{C}$ verändert (Abbildung 7.15b). Je Zyklus wurde eine Temperierzeit von $24 \mathrm{~h}$, zum Erreichen einer homogenen Temperaturverteilung in der gesamten Platte, gewählt.

In Abbildung 7.16 sind die Ergebnisse der experimentellen Untersuchung dargestellt. Dabei sind die zeitlichen Verläufe der Kerntemperaturen der Asphaltplatten mit und ohne PCM im Vergleich zur Lufttemperatur in der Temperierkammer zu sehen. Die Leistungsfähigkeit der Temperiereinheit ist sehr hoch, sodass sich die Zieltemperatur im Luftraum schnell einstellt. Diese sprunghafte Änderung führt zu einem kontinuierlichen Temperaturausgleich der Asphaltplatten an die neue Umgebungstemperatur. Für die Referenzvariante gilt, dass zu Beginn der Änderung der Lufttemperatur ein großer Temperaturgradient entsteht, welcher im Laufe der Zeit kleiner wird. Bei Temperaturgleichheit zwischen Kern- und Umgebungstemperatur ergibt sich keine weitere Veränderung. Der Einsatz von PCM beeinflusst das thermische Verhalten der Platte maßgebend. Beim Erwärmen der Asphaltplatte steigt auch die Kerntemperatur. Sobald die Temperatur des PCM den Schmelzpunkt von $58^{\circ} \mathrm{C}$ erreicht, setzt der Phasenwechsel ein. Dies führt dazu, dass die Kerntemperatur trotz höherer 


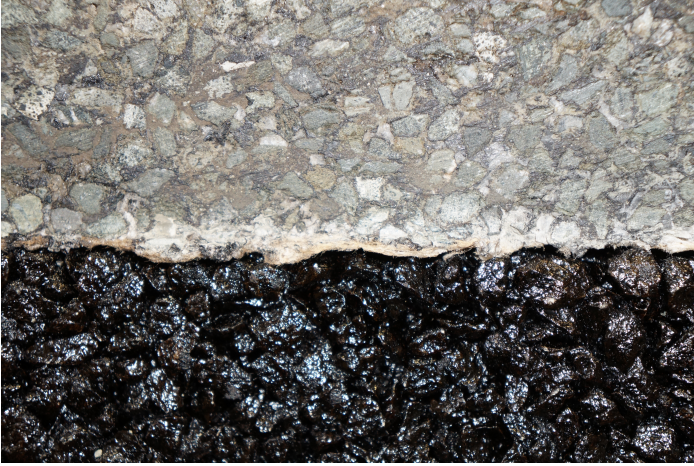

(a) oben Asphalt mit PCM, unten offenporige Referenzvariante

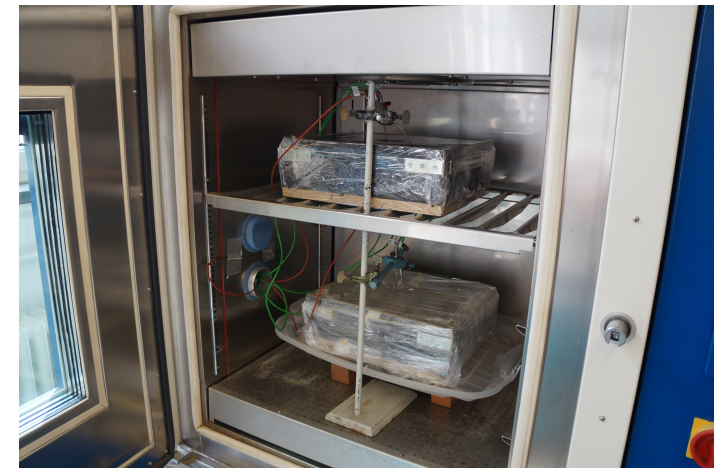

(b) Lagerung der Platten in der Temperierkammer

Abbildung 7.15

\section{Versuchsaufbau zur Untersuchung der Asphalt-PCM-Platte}

Umgebungstemperatur nur geringfügig weiter ansteigt. Dieser Zustand kann bei diesem Material für mehrere Stunden gemessen werden. Sobald die Phasenänderung von fest nach flüssig komplett abgeschlossen ist, steigt die Temperatur weiter an. Bedingt durch das Einbringen von PCM in die Hohlräume der Asphaltplatte ändern sich auch die mittleren thermophysikalischen Materialparameter (Wärmekapazität, Wärmeleitfähigkeit). Dies bewirkt zusätzlich ein verändertes Verhalten beim Erwärmen oder Abkühlen der Platte im gesamten Temperaturbereich. Beim Abkühlen der Asphaltplatte zeigt sich ein umgekehrtes Verhalten. Beim Erreichen der Erstarrungstemperatur erfolgt die Umwandlung von der flüssigen PCM in die feste Phase. Die gespeicherte Energie wird langsam an die Umgebung abgegeben. Dies führt dazu, dass die Temperatur um den Schmelzpunkt lange aufrechterhalten bleibt. Die gemessenen Zyklen zeigen, dass eine Mehrfachbeanspruchung des PCM keinen signifikanten Einfluss auf das thermische Verhalten hat. Die Zyklen sind harmonisch abgelaufen und demonstrieren die Einsatzfähigkeit des Konzeptes.

Mittels eines numerischen Modells wurden die Veränderungen der thermischen Bedingungen in Straßenkonstruktionen in Asphaltbauweise mit PCM berechnet. Die Simulationsergebnisse zeigen, dass die gewählte Schmelztemperatur der PCM einen erheblichen Einfluss auf die Temperaturzustände innerhalb der Straßenkonstruktion hat. In Abbildung 7.17 ist ein Ausschnitt der Simulationsergebnisse getrennt nach der Oberflächentemperatur und der Temperatur an der Oberseite der Asphalttragschicht dargestellt. Die Temperaturen der ersten beiden Tage liegen meist unter $30^{\circ} \mathrm{C}$ und somit sind die Verläufe aller untersuchten Varianten nahezu gleich. Nur die Variante mit dem Schmelzpunkt von $30^{\circ} \mathrm{C}$ ergibt minimale Abweichungen. Die simulierten Temperaturen ab dem 14.08. steigen erheblich an und die Wirkung der einzelnen PCM können deutlich festgestellt werden. Die PCM wurden bei den Simulationen in die Asphaltbinderschicht berücksichtigt. Sobald die Temperatur innerhalb der Schicht das Gebiet der Phasenwechseltemperatur erreicht hat, beginnt die Einlagerung der Wärme im Phasenwechsel. Dieser Effekt bewirkt eine Minderung der resultierenden 


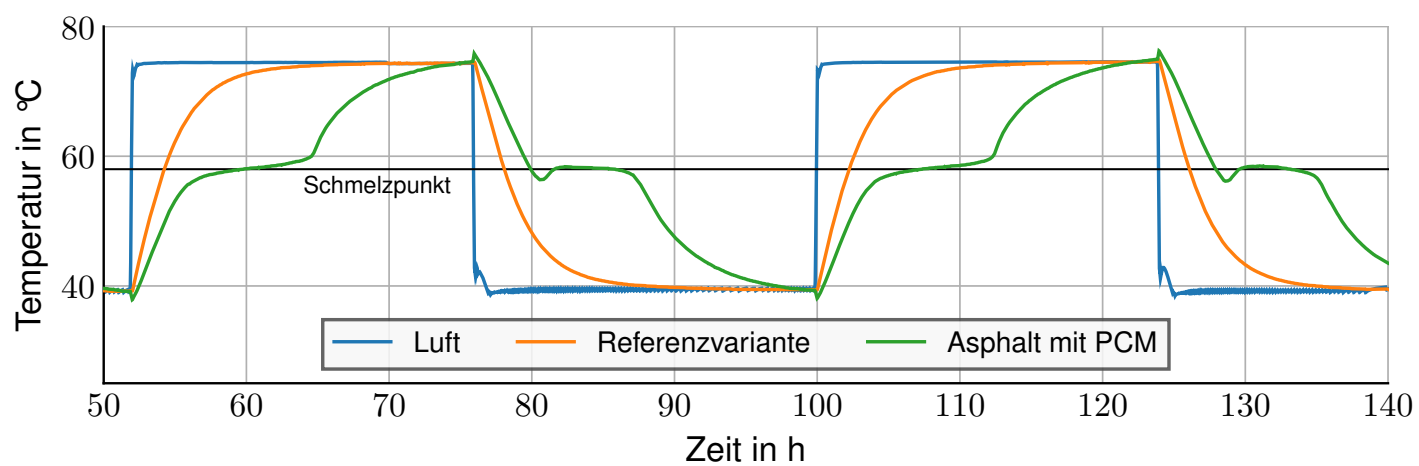

Abbildung 7.16

Temperatur innerhalb der Asphaltproben und der Temperierkammer in Abhängigkeit der Dauer des Experimentes

Temperaturen innerhalb der Straßenkonstruktion um die Mittagszeit. Dieses Verhalten kann sowohl in der Asphalttrag-, Asphaltbinder- als auch in der Asphaltdeckschicht festgestellt werden. Sinkt die Temperatur des Asphaltes unter den Schmelzpunkt des PCM oder kann das Material keine weitere thermische Energie binden, gibt das PCM Wärme an die umgebenden Stoffe ab. Dieses Phänomen kann aus den Temperaturgängen der Simulationsergebnisse, vor allem in den Nachtstunden, abgeleitet werden. Die Straßenkonstruktion kühlt nicht so schnell und stark ab, sodass die Temperaturen zu Beginn der nachfolgenden Tage leicht höher als bei der Referenzvariante sind. Trotz der geringeren nächtlichen Auskühlung liegen die Spitzenwerte der Varianten mit einem Schmelzpunkt kleiner gleich $40{ }^{\circ} \mathrm{C}$ an Tagen mit hoher thermischer Belastung der Straßenkonstruktion stets unterhalb der Temperatur der Referenzvariante.

Bestimmt man aus den Daten die Häufigkeitsverteilung in Abhängigkeit von der Temperatur (Abbildung 7.18), so ist ersichtlich, dass die Modifikationen nicht nur im Bereich der spezifischen Schmelzpunkte der PCM liegen, sondern das gesamte Spektrum mit Temperaturen größer als $15^{\circ} \mathrm{C}$ beeinflussen. Die Darstellung des Gesamtverlaufs (linke Diagramme) der Asphaltdeckschicht zeigt für die Variante mit einem Schmelzpunkt von $30^{\circ} \mathrm{C}$ eine deutliche Erhöhung des Auftretens von Temperaturen im Bereich des spezifischen Schmelzpunktes. Dies ist auf die Fähigkeit zur Speicherung der thermischen Energie zurückzuführen. Die Ergebnisse der Variante zeigen jedoch auch, dass die Häufigkeiten im Temperaturbereich zwischen $15^{\circ} \mathrm{C}$ und $25^{\circ} \mathrm{C}$ deutlich sinken. Die Deckschicht kühlt in den Nachtstunden nicht so stark ab. Dieses Verhalten ist sowohl auf der Asphaltdeckschicht der Straßenbefestigung als auch an der Oberseite der Asphalttragschicht zu beobachten. Die Größe der Abweichung ist deutlich vom Schmelzpunkt des jeweiligen PCM abhängig. Der Einsatz eines PCM bewirkt im hohen Temperaturspektrum (rechte Diagramme) eine deutliche Reduzierung der Temperaturen. Die Projektionen ergeben, dass die Häufigkeiten der hohen Temperaturen sowie die Maximalwerte in der Asphaltdeckschicht als auch Asphalttragschicht sinken. Die Variante mit einem Schmelzpunkt von $40^{\circ} \mathrm{C}$ bewirkt, dass sowohl die maximalen 


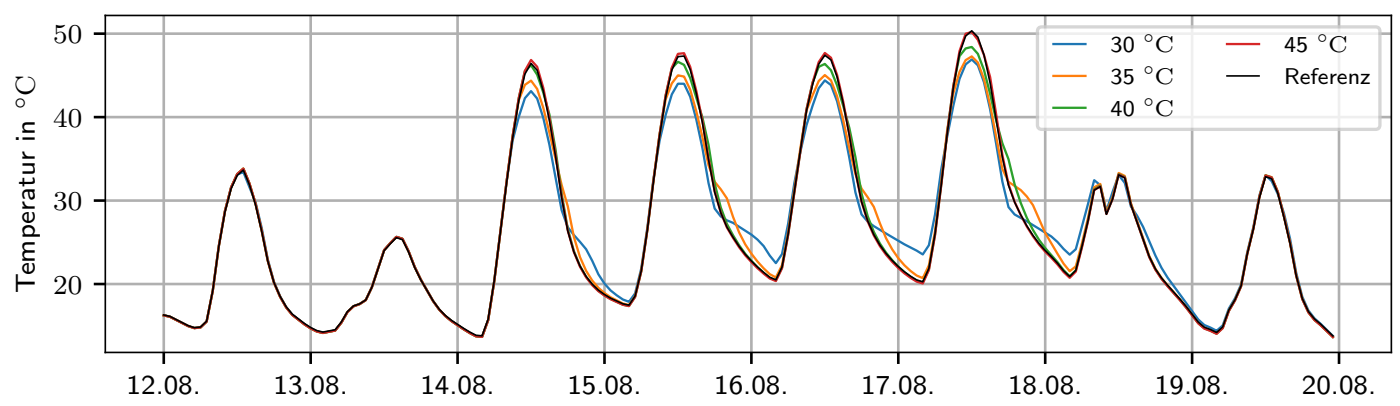

(a) Oberflächentemperatur

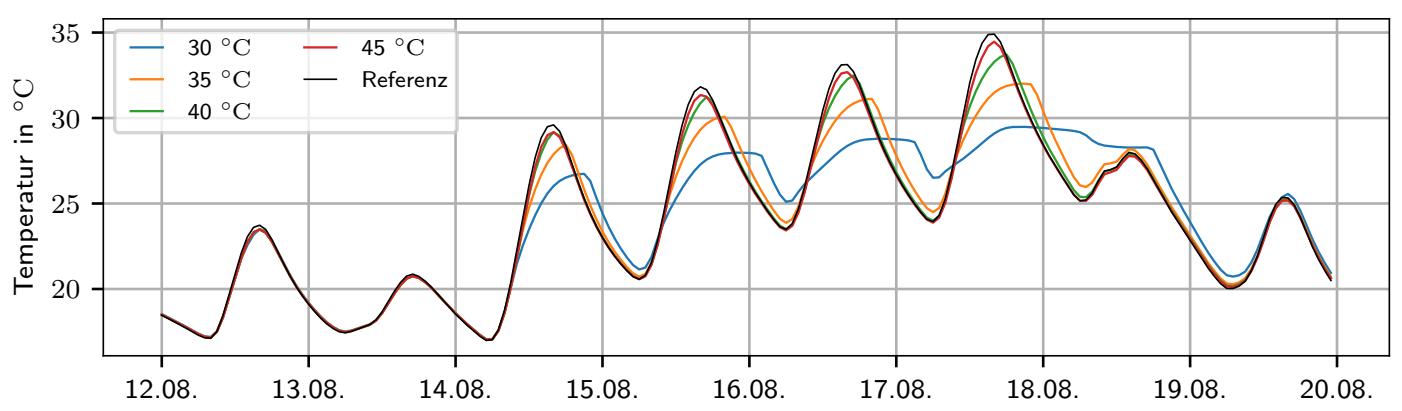

(b) Oberseite der Asphalttragschicht

Abbildung 7.17

Temperaturverlauf der einzelnen Variante mit unterschiedlichen Schmelzpunkten im Vergleich zur Referenzvariante, Ausschnitt

Temperaturen als auch die Häufigkeiten in der Asphaltdeck- und Asphalttragschicht am stärksten gemindert wurden. Diese Materialvariante hat den höchsten Einfluss auf das Temperaturverhalten bei sehr hohen Temperaturen und ist möglicherweise sehr gut geeignet, um die Spurrinnenentwicklung am stärksten zu mindern.

In Abbildung 7.19 ist die Beeinflussung der Temperaturzustände an der Oberseite der Fahrbahn in Abhängigkeit von der Tageszeit dargestellt. Die Diagramme geben die Abweichungen der Stunden an, in denen durch den Phasenwechsel der PCM eine Differenz der Temperaturen zwischen den Varianten mit PCM und der Referenzvariante besteht. Dabei wurden alle Temperaturzustände in Temperaturklassen in Abhängigkeit von der Tageszeit zugeordnet. Der Phasenwechsel der PCM führt zu einer Verschiebung innerhalb der Temperaturklassen. Diese Änderung wird mit der Verteilung der Referenzvariante verglichen. Die unterschiedlichen Schmelzpunkte haben einen direkten Einfluss auf die resultierenden Abweichungen. Die Variante mit einem Schmelzpunkt von $30^{\circ} \mathrm{C}$ hat den größten Einfluss auf das thermische Verhalten der Fahrbahnoberfläche. In den Mittagsstunden ergibt sich eine deutliche Reduktion der Temperaturen, wobei dies mit einer erhöhten Dauer der Tempera- 

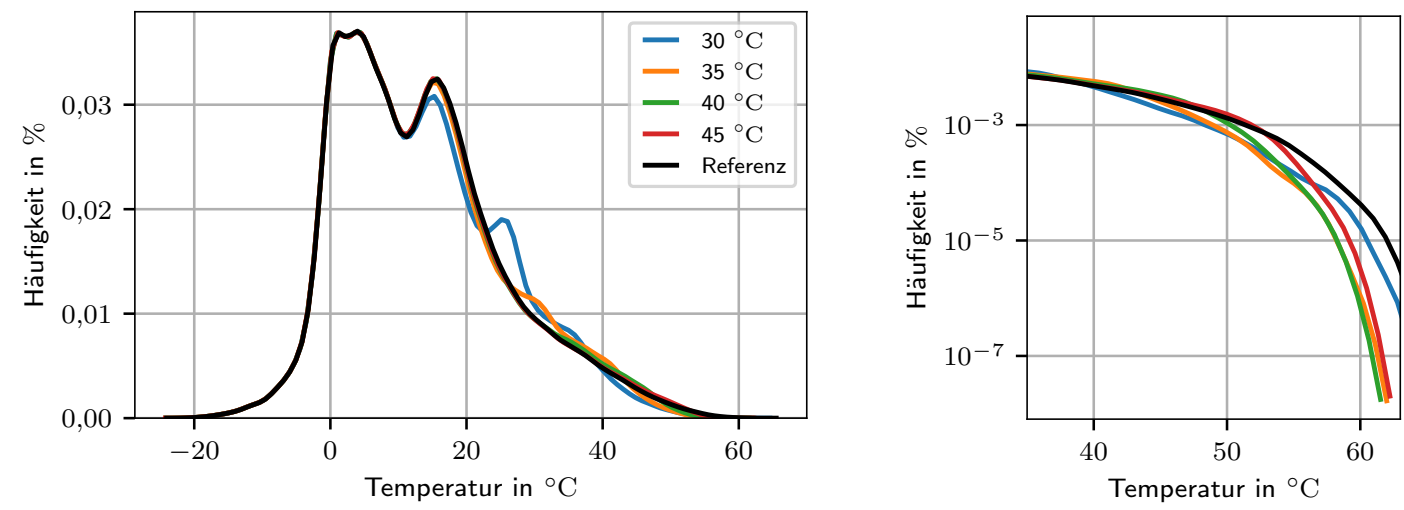

(a) Asphaltdeckschicht
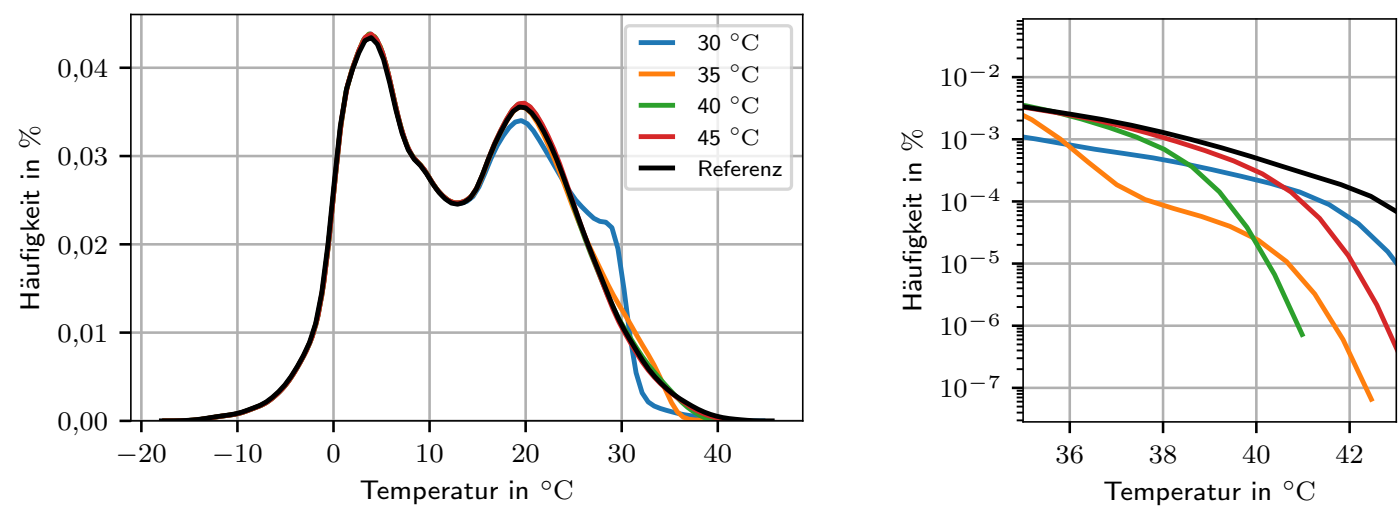

(b) Asphalttragschicht

Abbildung 7.18

Häufigkeitsverteilung der Kerntemperaturen der Referenzvariante und der Varianten mit PCM

turen um den Schmelzpunkt einhergeht. In den Nachtstunden kühlt die Straßenkonstruktion $a b$, wobei ein erneuter Phasenwechsel erfolgt und die gespeicherte Wärme an den Asphalt abgegeben wird. Dieser Effekt ist bei der Variante mit dem Schmelzpunkt von $30^{\circ} \mathrm{C}$ am deutlichsten ausgeprägt. Die weiteren Varianten haben ein identisches, jedoch weniger effektives Verhalten gezeigt. Dies führt dazu, dass im Laufe eines Jahres eine geringere Anzahl an Phasenwechseln erfolgt und die Temperaturen in den Nachtstunden nicht so stark beeinflusst werden. Die Aussagen gelten analog für die Analysen der Temperaturzustände an der Oberseite der Asphaltbinder- und Asphalttragschicht (siehe Anhang D.1).

Die Simulationen der thermischen Beanspruchungen haben ergeben, dass die Bewertung der Varianten mit dem Verfahren nach den RDO Asphalt (2009) möglich ist. Es muss jedoch davon ausgegangen werden, dass bei der Verwendung der konstanten Verkehrsverteilung über 


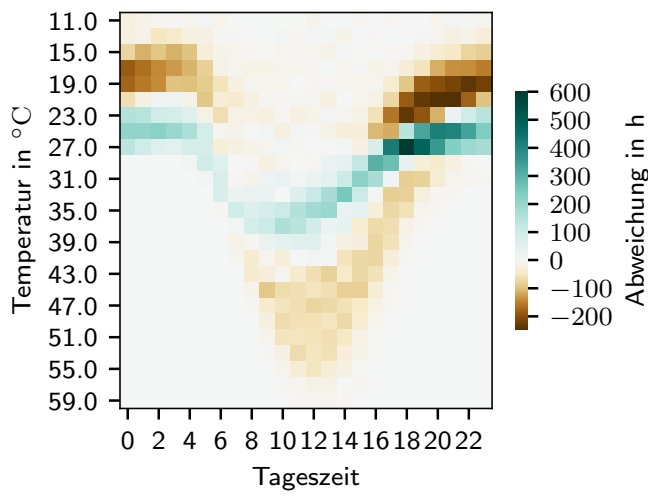

(a) Schmelzpunkt $30,00^{\circ} \mathrm{C}$

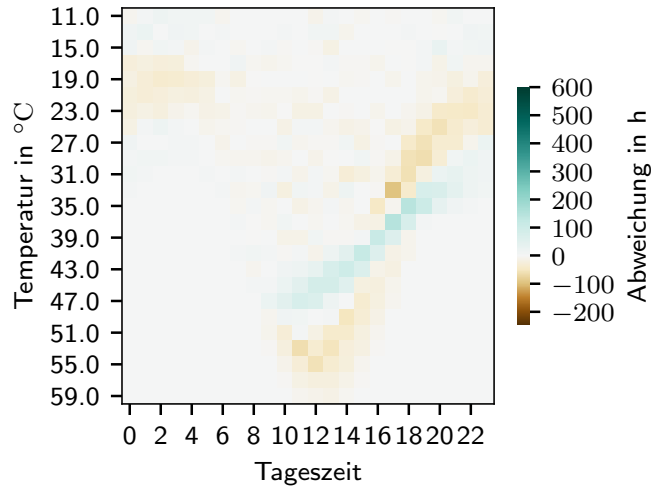

(c) Schmelzpunkt $40,00^{\circ} \mathrm{C}$

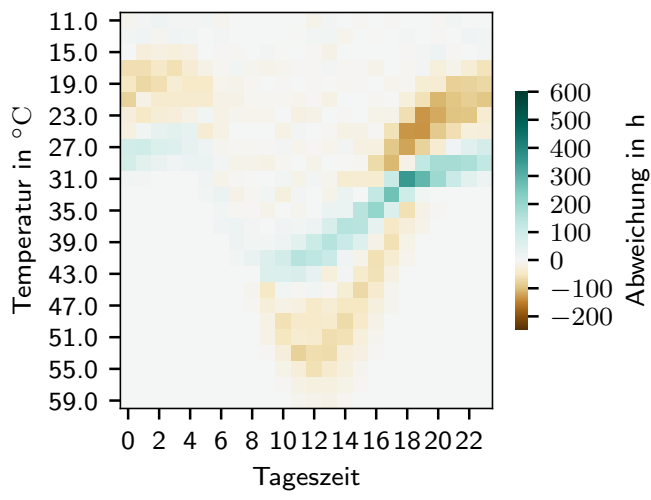

(b) Schmelzpunkt $35,00^{\circ} \mathrm{C}$

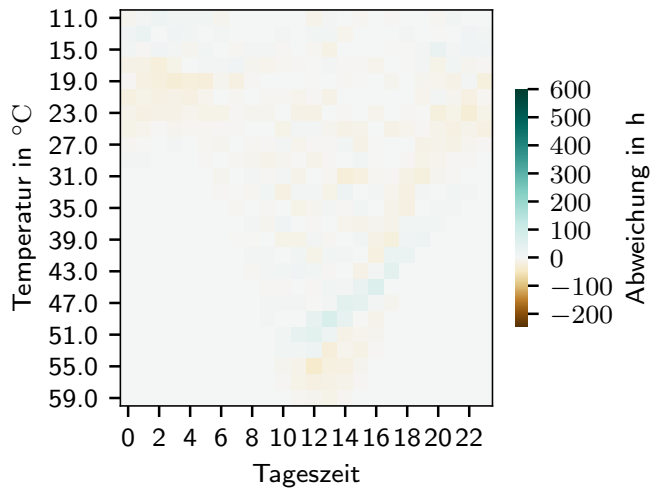

(d) Schmelzpunkt $45,00^{\circ} \mathrm{C}$

Abbildung 7.19

Akkumulierte stündliche Abweichung der Temperaturen auf der Oberseite einer Straßenkonstruktion in Abhängigkeit von der Tageszeit

den Tag eine Fehleinschätzung erfolgt. Die relativ gesehenen, höheren Temperaturen in der Nacht würden nach dem herkömmlichen Verfahren überproportional bewertet. Gleichzeitig erfolgt eine Minderung des Einflusses in den Mittagsstunden. Aus diesem Grund wurden für die Varianten die Mittelwertfunktionen bestimmt und die Schädigung der Straßenkonstruktion in Abhängigkeit von der Tageszeit auf Basis der Ganglinien des Verkehrs (siehe Abschnitt 3.5) berechnet. Da nur eine geringe Anzahl an Voruntersuchungen zur Bestimmung des Potenzials durchgeführt wurde, konnte das deutlich erweiterte Verfahren mit Berücksichtigung der Wochentage und Tageszeit angewendet werden.

Die einzelnen Ergebnisse der Berechnungen wurden jeweils relativ zum Ermüdungsstatus der Referenzvariante berechnet und als Abweichungen dazu dargestellt (Abbildung 7.20). Die Ergebnisse der Berechnungen deuten an, dass ein erhebliches Potenzial für die Verwendung 


\section{Fallbeispiele}

derartiger Stoffe besteht. Bei allen untersuchten Schmelzpunkten ergibt sich eine rechnerische Verringerung des Ermüdungsstatus, also kommt es zu einem späteren Zeitpunkt zu einer Rissentwicklung. Die Effekte in der Asphalttragschicht sind eher untergeordnet, da hier nur eine maximale Verringerung von 1,30\% berechnet wurde. Der Einfluss auf die Asphaltdeckschicht ist jedoch erheblich. Der Einsatz eines PCM mit einer Schmelztemperatur zwischen $30^{\circ} \mathrm{C}$ und $35^{\circ} \mathrm{C}$ hat hier einen deutlichen Einfluss auf die Dauerhaftigkeit. Entsprechend dieser Prognosen ist eine Verlängerung der Nutzungszeiträume von bis zu $40 \%$ möglich.
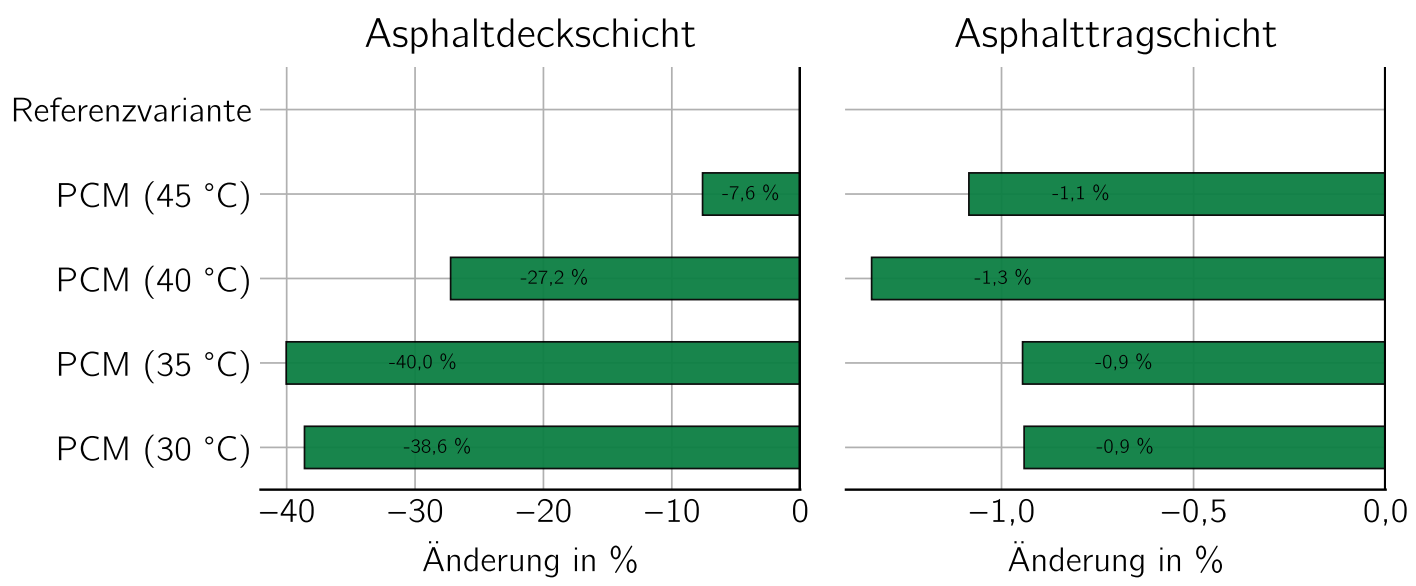

Abbildung 7.20

Relative Abweichung des Ermüdungsstatus zwischen den PCM-Varianten und der Referenzvariante unter Berücksichtigung der Tagesganglinie des Verkehrs

Der Einsatz von PCM in Asphalten verspricht eine kostengünstige Modifikation von Straßenbaumaterialien zur Optimierung der Gebrauchszeiträume von Straßenbefestigungen. Unter der Berücksichtigung des projizierten Klimawandels sollte an der Verwendung dieses Konzeptes weiter geforscht werden. Das Einbringen von PCM hat mit hoher Wahrscheinlichkeit einen Einfluss auf die Materialkennwerte von Asphalten. Möglicherweise ist das hier dargestellte Potenzial als zu groß anzusehen. Dies sollte dringend in weiteren Studien untersucht werden. 


\section{Zusammenfassung}

Hoher prognostizierter Zuwachs der Verkehrszahlen auf deutschen Straßen und immer extremer werdende Wetterbedingungen haben einen direkten Einfluss auf die Dauerhaftigkeit von Straßenkonstruktionen, insbesondere in Asphaltbauweise. Seit 2015 werden jährlich neue Hitzerekorde - die Temperaturanomalien in Europa übersteigen sogar die globalen Durchschnittswerte - gemessen. Diese Wetterextreme wurden bereits in der Vergangenheit mit Klimamodellen vorhergesagt. Die Geschwindigkeit, mit der die Prozesse sich jedoch aktuell entwickeln, übersteigt in vielen Bereichen die Ergebnisse aus früheren Studien (IPCC, 2012).

Die Zunahme der thermischen Belastungen in Kombination mit einem deutlich vergrößerten Schwerverkehrsaufkommen erfordert moderne Methoden für die Dimensionierung und Prognose von Straßenbefestigungen. Die Festlegung von Straßenaufbauten anhand von Standardwerten und Tabellen erscheint für hochbelastete Straßenkonstruktionen nicht mehr möglich. Die Anwendung von numerischen Modellen zur Vorhersage des mechanischen Verhaltens von Asphaltbefestigungen sollte mehrheitlich angewendet werden.

Für die rechnerische Dimensionierung und für Prognoserechnungen für Straßenkonstruktionen in Asphaltbauweise steht in Deutschland ein umfangreiches Regelwerk zur Verfügung (RDO Asphalt, 2009; RSO Asphalt, 2018). Dieses beschreibt die Methodik und definiert Eingangsdaten für die Durchführung der numerischen Berechnungen. Die Verfahren berücksichtigen dabei die konkreten mechanischen Materialparameter, die Verkehrsbelastungen, die Geometrie und die Temperaturzustände in den Straßenkonstruktionen. In Kapitel 3 wurden zwei Modifikationen des Regelwerks vorgeschlagen. Es konnte nachgewiesen werden, dass die vertikalen und horizontalen Dehnungskomponenten nicht für jeden Nachweispunkt der Straßenkonstruktion maßgebend sind. Die Verwendung der Hauptdehnungen berücksichtigt alle Komponenten des Verzerrungstensors, sodass die Maximalwerte der resultierenden Dehnungen im Berechnungsverfahren genutzt werden können. Dies hat einen erheblichen Einfluss auf die Ergebnisse der numerischen Berechnungen.

In Deutschland wird der Verkehrsfluss durch automatische Zählstationen auf Bundesautobahnen und Bundeslandstraßen an wichtigen Stellen erfasst. Auf Basis der ermittelten Daten konnte eine vereinfachte Tagesganglinie für die beiden Straßenkategorien abgeleitet werden. Bedingt durch die Datenlagen konnten zwei Funktionen für den KFZ-Verkehr und den Schwerverkehr ermittelt werden. Mittels dieser Erkenntnisse wurde das Berechnungsverfahren nach den RDO Asphalt (2009) erweitert und ein Vergleich mit einer linearen Tagesganglinie vorgenommen. Die Ergebnisse zeigen, dass ein signifikanter Unterschied zwischen den Verfahren festgestellt werden kann. Dies ist maßgeblich darauf zurückzuführen, 


\section{Zusammenfassung}

dass eine überproportionale Schädigung in den Mittagsstunden erfolgt. In diesem Zeitraum fahren auch die meisten Fahrzeuge der Klasse Schwerverkehr. In den Nachtstunden sinkt das Risiko gegenüber einer Schädigung. Es wird empfohlen, die vorhandenen Daten im Detail zu analysieren und Methoden zu entwickeln, um dezidierte Aussagen über eine möglichst hohe Bandbreite an Fahrzeugklassen und der Tageszeit des Auftretens ableiten zu können.

Durch Nachweise und Beispiele konnte gezeigt werden, dass die Adaption der vorgeschlagenen Erweiterungen einen erheblichen Vorteil bei der Durchführung von Dimensionierungsund Prognoserechnungen hat. Beide Erweiterungen sollten bei der Nachweisführung auf Ermüdungsrissbildung sowie Spurrinnenentwicklung Anwendung finden.

Für die Durchführung von Dimensionierungsrechnungen müssen charakteristische Temperaturzustände in Straßenkonstruktionen bekannt sein. Die klimatischen Bedingungen sowie die thermophysikalischen Materialparameter der eingesetzten Baustoffe ergeben zum Teil erhebliche Differenzen zwischen unterschiedlichen Berechnungsvarianten. Die Klimaprojektionen für Deutschland zeigen, dass die Jahresmitteltemperatur in Deutschland kontinuierlich ansteigen wird. Die zusätzlichen thermischen Belastungen haben einen direkten Einfluss auf die Temperaturbedingungen in Straßenkonstruktionen. Die Anzahl von heißen Tagen im Jahr, also Tagen mit einer maximalen Temperatur größer $30^{\circ} \mathrm{C}$, wird bis Ende des Jahrhunderts im Durchschnitt für die Asphaltdeckschicht mehr als $20 \%$ über den langjährigen Mittelwerten liegen, für die Asphalttragschicht beträgt diese Steigerung sogar $60 \%$. Die mittlere Temperatur steigt im Durchschnitt um über $1,50^{\circ} \mathrm{C}$ bezogen auf den Mittelwert aus 2020. Die Daten zeigen, dass Extremwerte innerhalb der Asphaltbefestigungen nicht nur im Sommer auftreten. Der Einfluss von Kälte muss bei der Konzipierung von Straßenbefestigungen weiterhin beachtet werden.

Die Veränderungen der thermischen Zustände innerhalb von Straßenkonstruktionen haben einen erheblichen Einfluss auf Asphaltbefestigungen. Bedingt durch die in Deutschland sehr großen klimatischen Unterschiede, erfolgt der beschriebene Prozess nicht gleichmäßig. Weiterhin haben Faktoren wie das Reflexionsvermögen der Oberflächen, die thermophysikalischen Materialparameter und die Geometrie einen zusätzlichen Einfluss auf die Temperaturbedingungen in Straßenkonstruktionen. Eine Verallgemeinerung zur Ableitung eines allgemeingültigen Anpassungsprozesses erscheint nicht möglich.

Die Berücksichtigung von stoffspezifischen Materialparametern bei der Berechnung der thermischen Zustände von Straßenbefestigungen und die Erkenntnis, dass möglichst geringe Abweichungen zwischen realen Temperaturbedingungen und statistischen Temperaturprofilen vorherrschen müssen, führt dazu, dass eine Weiterentwicklung der RDO Asphalt (2009) empfohlen wird. Die Untersuchungen in der vorliegenden Arbeit haben gezeigt, dass die Nutzung von standardisierten Temperaturprofilen zum Teil erhebliche Abweichungen zwischen den statistischen Temperaturzuständen und den Simulationswerten, besonders im Bereich der Asphalttragschicht, aufweisen. Die Verwendung der in dieser Arbeit entwickelten Methodik zur Analyse der thermischen Zustände von Straßenbefestigungen berücksichtigt die Geometrie und die thermophysikalischen Stoffparameter der Baustoffe der gesamten Straßenkonstruktion sowie die lokalen klimatischen Bedingungen. 
Die Bewertung der thermischen Zustände beruht auf einer Clusteranalyse des gesamten Temperaturspektrums der Nutzungsphase einer Straßenbefestigungen. Die Ergebnisse der Analysen bilden Cluster, welche die mittleren Eigenschaften der zugehörigen Temperaturzustände abbilden (Mittelwertfunktionen). Die Verwendung des Verfahres führt zu einer signifikanten Erhöhung der Güte der statistischen Betrachtung der Temperaturzuständen in Straßenbefestigungen. Weiterhin ist es möglich, die Methodik auch bei der Bewertung der ungebundenen Schichten von Straßenkonstruktion oder für die Beton- und Pflasterbauweise zu verwenden. Mit dem Verfahren ist es weiterhin möglich, Sonderbauweisen zu bewerten.

In einzelnen Studien konnte gezeigt werden, dass für die Straßeninfrastruktur in Asphaltbauweise ein erhebliches Risiko infolge des projizierten Klimawandels besteht. Die Vulnerabilität der Verkehrsinfrastruktur für ganz Deutschland wurde in dieser Arbeit auf Basis von umfänglichen Serienrechnungen bestimmt. Gegenstand der Berechnungen ist ein fiktiver Straßenaufbau mit repräsentativen Materialparametern für die Asphalte sowie sonstigen Baustoffe. Weiterhin wurde eine einheitliche Belastung durch den Verkehr für alle Berechnungen verwendet. Dieses Vorgehen ermöglicht einen Vergleich der Risiken für einzelne Gebiete.

Auf Basis der Simulationsergebnisse wurde der Ermüdungsstatus im Betrachtungsintervall von 1960 bis 2100 für ganz Deutschland bestimmt. Die Ergebnisse dieser Serienrechnungen zeigen, dass in der Vergangenheit ein signifikant geringeres Risiko gegenüber einer Ermüdungsrissbildung für das gesamte Bundesgebiet bestand. In wenigen Teilen Deutschlands, meist im Süd-Westen, konnten leicht erhöhte Werte detektiert werden. Mit Fortschreiten des Klimawandels verringert sich die schadensfreie Nutzungsdauer von Straßenkonstruktionen in Asphaltbauweise. Bis Ende des Jahrhunderts wird die mittlere Nutzungsdauer einer Straßenbefestigung in großen Teilen Deutschlands um 25\% und in einem erheblichen Flächenanteil um $50 \%$ im Vergleich zum Flächenmittelwert aller Ergebnisse aus 2020 sinken (Abbildung 6.2).

In dieser Arbeit wurden weiterhin Berechnungen zur Spurrinnenentwicklung durchgeführt. Basierend auf den Ergebnissen muss festgestellt werden, dass auch unter diesem Aspekt eine signifikante Zunahme der Schädigungen zu erwarten ist. Die örtliche Verteilung gilt analog zu den vorab beschriebenen Erkenntnissen (Abbildung 6.3).

Zusammenfassend kann festgestellt werden, dass die Vulnerabilität der Verkehrsinfrastruktur aus Asphalt bedingt durch den Klimawandel signifikant steigen wird. Anhand der Ergebnisse dieser Arbeit muss davon ausgegangen werden, dass eine erhebliche Verkürzung der Nutzungszeiträume von Asphaltbefestigungen durch die klimatischen Effekte erfolgen wird. Eine Verringerung der negativen Auswirkungen erfordert eine Erarbeitung einer Anpassungsstrategie an den Klimawandel und eine rasche Umsetzung im Verfahren zur Dimensionierung von Straßenbefestigungen.

Im Jahr 2019 wurde die aktualisierte Fassung der KiST-Zonen-Karte vorgestellt (Augter und Kayer, 2019). Diese umfasst eine deutlich feinere Einteilung des Bundesgebietes in Klassen mit ähnlichen Temperaturzuständen. Grundlegend sind die Erkenntnisse zwischen 


\section{Zusammenfassung}

den Methoden identisch, jedoch beruhen die Berechnungen für die KiST-Zonen-Karte auf Messdaten der Vergangenheit. Die klimatischen Bedingungen in Deutschland haben sich jedoch in den letzten Jahren stark verändert und Projektionen des zukünftigen Klimas zeigen, dass dies gerade der Anfang einer langjährigen Veränderung der klimatischen Bedingungen ist. Nur die Verwendung von Klimaprojektionen, im besten Fall von Ensemble-Rechnungen vieler regionaler Klimamodelle, ermöglicht eine Erstellung einer geeigneten Anpassungsstrategie. Die Konstruktionsweise und Konzipierung von Straßenkonstruktionen müssen an die sich rasch ändernden Bedingungen angepasst werden. Ein Zögern führt zu einer Minderung der Qualität der Infrastruktur unter gleichzeitig hohen Kosten für die Instandsetzung und Erneuerung. 


\section{Literaturverzeichnis}

Alexiades, Vasilios und Alan D. Solomon (1993). Mathematical modeling of melting and freezing processes. Washington: Hemisphere Publ. XIII, 323. ISBN: 1-56032-125-3 (siehe S. 33-34).

Arrhenius, Svante August (1896). "On the influence of carbonic acid in the air upon the temperature of the ground". In: The London, Edinburgh and Dublin Philosophical Magazine and Journal of Science 5, S. 237-276 (siehe S. 8).

Augter, Gisela und Sascha Kayer (2019). KiST-Zonen-Karte RDO und RSO Asphalt. Techn. Ber. Berichte der Bundesanstalt für Straßenwesen, S. 74 (siehe S. 101).

Backhaus, Klaus u. a. (2016). Multivariate Analysemethoden - Eine anwendungsorientierte Einführung. Bd. 14, S. 854. ISBN: 9783540850441. DOI: 10.1007/3-540-29932-7 (siehe S. 53).

Bador, Margot u. a. (2015). „Spatial clustering of summer temperature maxima from the CNRM-CM5 climate model ensembles \& E-OBS over Europe". In: Weather and Climate Extremes 9, S. 17-24. ISSN: 22120947. DOI: 10.1016/j.wace.2015.05.003 (siehe S. 57).

Brasseur, Guy P., Daniela Jacob und Susanne Schuck-Zöller (2017). Klimawandel in Deutschland - Entwicklung, Folgen, Risiken und Perspektiven. Berlin, Heidelberg: Springer Berlin Heidelberg. ISBN: 9783662503966. DOI: 10.1007/978-3-662-50397-3_8 (siehe S. 7, 12-13).

Cabeza, Luisa F. u. a. (2011). „Materials used as PCM in thermal energy storage in buildings: A review". In: Renewable and Sustainable Energy Reviews 15.3, S. 1675-1695 (siehe S. 91).

Cao, Xuejuan u.a. (2011). „Cooling principle analyses and performance evaluation of heat-reflective coating for asphalt pavement". In: Journal of materials in civil engineering 23.7, S. 1067-1075 (siehe S. 91).

Carvalho, M. J. u. a. (2016). „Regionalization of Europe based on a K-Means Cluster Analysis of the climate change of temperatures and precipitation". In: Physics and Chemistry of the Earth 94, S. 22-28. ISSN: 14747065. DOI: 10.1016/j.pce.2016.05.001 (siehe S. 55,57$)$.

Chen, Mei Zhu u. a. (2011). „Optimization of phase change materials used in asphalt pavement to prevent rutting". In: Advanced materials research. Bd. 219. Trans Tech Publ, S. 1375-1378 (siehe S. 91).

Chen, Meizhu, Lu Wan und Juntao Lin (2012). „Effect of phase-change materials on thermal and mechanical properties of asphalt mixtures". In: Journal of Testing and Evaluation 40.5, S. 746-753 (siehe S. 91).

Clauß, Markus, Sascha Kayser und Frohmut Wellner (Aug. 2017). „Projizierter Klimawandel und Dimensionierung von Straßenbefestigungen“. In: Forschung Straßenbau und Straßenverkehrstechnik 1128 (siehe S. 2, 13-14, 65).

Clauß, Markus u. a. (2017). NANOASPHALT - Optimierung der Gebrauchseigenschaften und der Beständigkeit von Asphaltstraßen unter Nutzung der Nanotechnologie. Techn. Ber. (siehe S. 20). 


\section{Zusammenfassung}

Clauß, Markus u. a. (2019). Untersuchung multifunktionaler Straßenbaumaterialien und Verbundwerkstoffe zur Nutzung solarer Energie und Verbesserung der Dauerhaftigkeit. Techn. Ber. Dresden: Techische Universität Dresden, S. 159. DOI: https://doi.org /10.2314/KXP: 1671420187 (siehe S. 31-32, 35, 37, 67, 75, 77, 83-84).

Dragon, Ines und Uwe Reinhardt (2015). „Weiterentwicklung der Ansätze für die Spurrinnenprognose von Asphaltbefestigungen". In: Straße + Autobahn 4 (siehe S. 1920).

Euronews (Nov. 2019). „Europäische Städte müssen sich für häufigere Hitzewellen wappnen“. In: Euronews (siehe S. 3).

Fischer, E. M. und C. Schär (2010). „Consistent geographical patterns of changes in high-impact European heatwaves". In: Nature Geoscience. ISSN: 17520894. DOI: 10.1038/ngeo866 (siehe S. 2).

Gudipudi, Padmini P., B. Shane Underwood und Ali Zalghout (Dez. 2017). „Impact of climate change on pavement structural performance in the United States". In: Transportation Research Part D: Transport and Environment 57, S. 172-184. ISSN: 1361-9209. DOI: 10.1016/J.TRD. 2017.09.022 (siehe S. 2, 14).

Hartmann, Dennis L. u. a. (Jan. 2013). „Observations: Atmosphere and surface“. English (US). In: Climate Change 2013 the Physical Science Basis: Working Group I Contribution to the Fifth Assessment Report of the Intergovernmental Panel on Climate Change. Bd. 9781107057999. Cambridge University Press, S. 159-254. ISBN: 9781107415324. DOI: 10.1017/CB09781107415324.008 (siehe S. 2).

Harvey, Chelsea (Aug. 2017). „Unchecked climate change could bring extremely dangerous heat to South Asia, scientists warn". In: The Washington Post (siehe S. 3).

IPCC (2007). „Climate Change 2007 : Summary for Policymakers“. In: Hemisphere. Hrsg. von Lenny Bernstein u. a. (siehe S. 9).

- (2012). Zusammenfassung für politische Entscheidungsträger. In: Management des Risikos von Extremereignissen und Katastrophen zur Förderung der Anpassung an den Klimawandel. Field, C. Genf, Schweiz, S. 24. ISBN: 978-3-00-039367-9 (siehe S. 99).

- (2013). „Summary for Policymakers“. In: Climate Change 2013: The Physical Science Basis. Contribution of Working Group I to the Fifth Assessment Report of the Intergovernmental Panel on Climate Change. Hrsg. von T.F. Stocker u. a. Cambridge, United Kingdom und New York, NY, USA: Cambridge University Press. Kap. SPM, S. 1-30. ISBN: ISBN 978-1-107-66182-0. DOI: 10.1017/CB09781107415324.004 (siehe S. 10$11)$.

- (2014). Climate Change 2014: Synthesis Report. Contribution of Working Groups I, II and III to the Fifth Assessment Report of the Intergovernmental Panel on Climate Change. Pachauri, R.K. and Meyer, L. A. Genf, Schweiz, S. 151. ISBN: 978-92-9169-143-2 (siehe S. 2).

- (2018). "Summary for Policymakers". In: Global Warming of $1.5^{\circ} \mathrm{C}$. An IPCC Special Report on the impacts of global warming of $1.5^{\circ} \mathrm{C}$ above pre-industrial levels and related global greenhouse gas emission pathways, in the context of strengthening the global response to the threat of climate change, hrsg. von $\mathrm{V}$. Masson-Delmotte, $\mathrm{H}$. O. Portner 
und D. Roberts. Geneva, Switzerland: World Meteorological Organization, S. 32. ISBN: 9788578110796. DOI: 10.1017/CB09781107415324.004 (siehe S. 12).

Jacob, Daniela (2005a). REMO A1B SCENARIO RUN, UBA PROJECT , 0.088 DEGREE RESOLUTION, RUN NO. 006211, 1H DATA (siehe S. 35, 66).

- (2005b). REMO climate of the 20th century run - 0.088 degree resolution run no. 006210, 1 h data. Techn. Ber. (siehe S. 79).

Jacob, Daniela, Hinnerk Ries und Björn Weber (2016). Entwicklung von Extremwetterereignissen in Deutschland. Techn. Ber. Climate Service Center Germany, S. 8 (siehe S. 6).

Kaspar, F. und K. Friedrich (2020). Rückblick auf die Temperatur in Deutschland im Jahr 2019 und die langfristige Entwicklung. Techn. Ber. Deutscher Wetterdienst, S. 1-6 (siehe S. 6, 86).

Kayser, Sascha (2007). „Einflüsse für Dimensionierungsrechnungen von Asphaltbefestigungen“. Dissertation. Technische Universität Dresden (siehe S. 13, 53, 58-59, 61, 80).

Kayser, Sascha, Uwe Reinhardt und Alexander Zeißler (2011). „Spurrinnenprognose für Asphaltbefestigungen unter Berücksichtigung von Zufallsprozessen“. In: Dresdner As phalttrage 2011. Dresden, S. 7-18 (siehe S. 19-20).

Killick, R., P. Fearnhead und I. A. Eckley (2012). „Optimal Detection of Changepoints With a Linear Computational Cost". In: Journal of the American Statistical Association 107.500, S. 1590-1598. DOI: 10.1080/01621459.2012.737745 (siehe S. 50).

Lletí, R. u. a. (2004). „Selecting variables for k-means cluster analysis by using a genetic algorithm that optimises the silhouettes". In: Analytica Chimica Acta 515.1, S. 87-100. DOI: 10.1016/j.aca.2003.12.020 (siehe S. 53).

Lozan, José u. a. (2014). „Die Auswirkungen von thermischen Belastungen auf die Mortalität". In: Warnsignale Klima: Gesundheitsrisiken. Gefahren für für Pflanzen, Tiere und Menschen (siehe S. 2).

Lüthi, Dieter u. a. (2008). „High-resolution carbon dioxide concentration record 650,000800,000 years before present". English. In: Nature 453.7193, S. 379-82. ISSN: 0028-0836. DOI: $10.1038 /$ nature06949 (siehe S. 11).

Mahlstein, Irina und Reto Knutti (2009). „Regional climate change patterns identified by cluster analysis". In: Climate Dynamics 35.4, S. 587-600. DOI: 10.1007/s00382-0090654-0 (siehe S. 13).

Manaba, S. und F. Möller (1961). „On the radiative equilibrium and heat balance of the atmosphere". In: Monthly Weather Review 31:118-133 31, S. 118-133 (siehe S. 8).

Miner, M.A. (1945). "Cummulative damage in fatigue“. In: Journal of Applied Mechanics 12.3 (siehe S. 18).

NASA (2020). GISS Surface Temperature Analysis (v4). URL: https://data.giss.nasa .gov/gistemp/graphs_v4 (besucht am 14.07.2020) (siehe S. 1).

Neubauer, Dirk (Juli 2015). „Düsseldorf schmilzt dahin: Asphalt so weich wie Pudding Sperrungen auf Gruner- und Spichernstraße". In: www.report-d.de (siehe S. 2-3).

Neutag, Lars (2005). „Berücksichtigung verschiedener Alterungsstufen für die Ermüdung von Asphalttragschichtmischgut auf die analytische Bemessung im Rahmen von Funk- 


\section{Zusammenfassung}

tionsbauverträgen/ Betreibermodellen“. Diss. Bergische Universität Wuppertal (siehe S. 15).

Numrich, Ralf (2003). „Modellierung des nichtlinear-elastischen Verformungsverhaltens von Tragschichten ohne Bindemittel". Diss. Technische Universität Dresden (siehe S. 15).

Pham, D. T., S. S. Dimov und C. D. Nguyen (2005). „Selection of K in K-means clustering“. In: Proceedings of the Institution of Mechanical Engineers, Part C: Journal of Mechanical Engineering Science 219.1, S. 103-119. ISSN: 09544062. DOI: 10.1243/095440605X8 298 (siehe S. 57).

Qiao, Yaning u. a. (Dez. 2015). „Evaluating the effects of climate change on road maintenance intervention strategies and Life-Cycle Costs". In: Transportation Research Part D: Transport and Environment 41, S. 492-503. ISSN: 1361-9209. DOI: 10.1016/J.TRD.2 015.09 .019 (siehe S. 2).

Robine, Jean Marie u. a. (2008). „Death toll exceeded 70,000 in Europe during the summer of 2003". In: Comptes Rendus - Biologies. ISSN: 16310691. DOI: 10.1016/j.crvi.20 07.12 .001 (siehe S. 2).

Rousseeuw, Peter J (1987). „Silhouettes: a graphical aid to the interpretation and validation of cluster analysis". In: Journal of Computational and Applied Mathematics 20, S. 53-65. ISSN: 03770427. DOI: 10.1016/0377-0427(87)90125-7 (siehe S. 57).

Schoetter, Robert u. a. (Apr. 2012). „Evaluation and Bias Correction of Regional Climate Model Results Using Model Evaluation Measures". In: Journal of Applied Meteorology and Climatology 51.9, S. 1670-1684. ISSN: 1558-8424. DOI: 10.1175/JAMC-D-11-0161.1 (siehe S. 13).

Sharma, Atul u. a. (2009). „Review on thermal energy storage with phase change materials and applications". In: Renewable and Sustainable energy reviews 13.2, S. 318-345 (siehe S. 91).

Stocker, Thomas F. u. a. (2013). Climate change 2013 the physical science basis: Working Group I contribution to the fifth assessment report of the intergovernmental panel on climate change. ISBN: 9781107415324. DOI: 10.1017/CB09781107415324 (siehe S. 10).

Uhlig, Wolf (2019). „Grundlagen für Lastannahmen zur Dimensionierung von Straßenbefestigungen“. Dissertation. Technischen Universität Dresden (siehe S. 24).

VDI e. V., Hrsg. (2013). VDI-Wärmeatlas. 11., bearbeitete und erweiterte Auflage. Berlin u. a.: Springer-Verlag. ISBN: 978-3-642-19980-6 (siehe S. 35).

Viola, Francesco und Clara Celauro (Juni 2015). „Effect of climate change on asphalt binder selection for road construction in Italy". In: Transportation Research Part D: Transport and Environment 37, S. 40-47. ISSN: 1361-9209. DOI: 10.1016/J. TRD. 2015.04.012 (siehe S. 2, 14).

Vuuren, Detlef P. u. a. (Aug. 2011). "The representative concentration pathways: an overview". In: Climatic Change 109.1-2, S. 5-31. ISSN: 0165-0009. DOI: 10.1007/s10 584-011-0148-z (siehe S. 9).

Wayne, Graham. (2013). The Beginner's Guide to Representative Concentration Pathways. Techn. Ber. (siehe S. 9). 
Wetschera, Wiebke (Juli 2019). „Wie die Hitzewelle unseren Verkehr einschränkt”. In: Südkurier (siehe S. 3).

Wistuba, Michael P. und Axel Walther (2013). "Consideration of climate change in the mechanistic pavement design". In: Road Materials and Pavement Design. ISSN: 21647402. DOI: 10.1080/14680629.2013.774759 (siehe S. 2, 13).

Yan, Mingjin (2005). "Methods of Determining the Number of Clusters in a Data Set and a New Clustering Criterion“. Dissertation. Virginia Polytechnic Institute und State University in (siehe S. 57).

\section{Normenverzeichnis}

RDO Asphalt (Aug. 2009). Richtlinien für die rechnerische Dimensionierung von Verkehrsflächen mit Asphaltdeckschicht. Forschungsgesellschaft für Straßen- und Verklehrswesen e. V. (siehe S. ii, 13, 15-18, 24, 26-27, 29, 53, 58-62, 65-67, 96, 99-100).

RSO Asphalt (2018). Richtlinien zur Bewertung der strukturellen Substanz des Oberbaus von Verkehrsflächen in Asphaltbauweise (Entwurf). Forschungsgesellschaft für Straßenund Verklehrswesen e. V. (siehe S. 15, 99).

RStO 12 (2012). Richtlinien für die Standardisierung des Oberbaus von Verkehrsflächen. Forschungsgesellschaft für Straßen- und Verklehrswesen e. V. (siehe S. 32, 39, 75, 77). TP Asphalt-StB Teil 6 (2007). Technischen Prüfvorschriften für Asphalt - Teil 6: Raumdichte von Asphalt-Probekörpern (siehe S. 37). 



\section{Abkürzungsverzeichnis}

BASt Bundesanstalt für Straßenwesen. 25

CLM Climate Local Model. 12, 13

duraBASt Demonstrations-, Untersuchungs- und Referenzareal der Bundesanstalt für Straßenwesen. 78

FEM Finite Elemente Methode. 16

ForProS Forschungsprogramm Straßenbau. 16

FVM Finite Volumen Methode. 32

IPCC Intergovernmental Panel of Climate Change. 8, 9

NCEI National Centers for Environmental Information. 2

ncTs normierte charakteristische Temperaturprofile. 29, 53, 58-60, 62, 80, 83, 85, 86

NOAA National Oceanic and Atmospheric Administration. 2

PCM Phase Change Materials. 91-95, 98

RCP Representative Concentration Pathways. 9, 10

REMO REgional MOdell. 12, 13

WMO World Meteorological Organization. 5 



\section{Symbolverzeichnis}

\begin{tabular}{ccll}
\hline Symbol & Einheit & Bezeichnung & Seite(n) \\
\hline$A$ & $\mathrm{~m}^{2}$ & Fläche der Fahrbahn & 34 \\
$A_{i}$ & $\mathrm{~m}^{2}$ & Querschnittsfläche des Zellvolumens & 33,34 \\
$\alpha$ & - & Albedo & 34 \\
$\alpha_{\mathrm{K}}$ & $\mathrm{W} \mathrm{m}^{-2} \mathrm{~K}^{-1}$ & Wärmeübergangskoeffizienten & 34,35 \\
$\epsilon$ & - & Emissionsgrad & 34 \\
$\Delta x_{i}$ & $\mathrm{~m}$ & Position des Mittelpunktes eines Zellvolumens & 33 \\
$\dot{q}_{\mathrm{AG}}$ & & Wärmestromdichte der athmosphärischen & 34 \\
& & Gegenstrahlung & \\
$\lambda$ & $\mathrm{W} \mathrm{m}{ }^{-1} \mathrm{~K}$ & Wärmeleitfähigkeit & 33,35 \\
$L$ & $\mathrm{~m}$ & Charakteristische Länge & 35 \\
$N u$ & & Nusselt-Zahl & 35 \\
$\dot{Q}_{i \pm 1 / 2}$ & $\mathrm{~W}$ & Wärmestrom zu den angrenzenden Zellvolumen & 33 \\
$\dot{q}_{\mathrm{G}}$ & $\mathrm{W} / \mathrm{m}^{2}$ & Wärmestromdichte der Globalstrahlung & 34 \\
$\dot{Q}_{\mathrm{K}}$ & $\mathrm{W}$ & konvektiver Wärmestrom & 34 \\
$\varrho$ & $\mathrm{g} / \mathrm{cm}^{3}$ & Dichte & 33 \\
$\sigma$ & $\mathrm{W} \mathrm{m}{ }^{-2} \mathrm{~K}^{-4}$ & Stefan-Boltzmann-Konstante & 34 \\
$R_{i \pm 1 / 2}$ & $\mathrm{~K}$ & Wärmewiderstände zwischen zwei Zellmittelpunkten & 33 \\
$T$ & $\mathrm{~K}$ & Temperatur & 33,34 \\
$T_{\mathrm{L}}$ & $\mathrm{K}$ & Temperatur der Luft & 34 \\
$T_{\mathrm{OF}}$ & $\mathrm{K}$ & Temperatur der Fahrbahnoberfläche & 34 \\
\hline
\end{tabular}





\section{Abbildungsverzeichnis}

1.1 Temperaturanomalie der Oberflächentemperatur der Erde bezogen auf die Periode 1980 bis $2015 \ldots \ldots$. . . . . . . . . . . . . . . . 1

1.2 Beispiele für Schädigungen durch Hitze an der Verkehrsinfrastruktur . . . . 3

2.1 Anomalie der Lufttemperatur für den Zeitraum von 1881 bis 2019 (Deutscher Wetterdienst) . . . . . . . . . . . . . . . . . . 6 6

2.2 Anomalie der Anzahl der Sommertage für Deutschland (Deutscher Wetterdienst) . . . . . . . . . . . . . . . . . . . . 7

2.3 Darstellung der Treibhausgas-Emissionen nach den SRES-Emissionsszenarien und der resultierenden Temperaturanomalie . . . . . . . . . . . . . . . 9

2.4 Veränderung des Strahlungsantriebes der Atmosphäre im Vergleich zwischen den SRES-Emissionsszenarien zu den RCPs . . . . . . . . . . . . . . . . . 10

2.5 Globale Temperaturänderung relativ zum Mittelwert der Periode 1850 bis 1900, historische Werte . . . . . . . . . . . . . . . . . . . . . . 11

2.6 Globale Temperaturänderung relativ zum Mittelwert der Periode 1850 bis

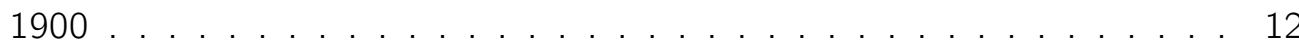

2.7 Entwicklung der Dauerhaftigkeit von Straßenkonstruktionen in Abhängigkeit der Verkehrsfreigabe . . . . . . . . . . . . . . . . . . . . . . . . . 14

3.1 Ablaufschema über die Arbeitsschritte bei der rechnerischen Dimensionierung von Straßenbefestigungen . . . . . . . . . . . . . . . . . . . . . 16

3.2 Verteilung der Hauptdehnungen innerhalb einer Straßenkonstruktion, Beispiele aus Serienrechnungen . . . . . . . . . . . . . . . . . . . . . . . 22

3.3 Lage der Nachweispunkte sowie Richtung und Größe (Länge der Pfeile) der Hauptdehnungen, dargestellt ist der Maximalwerte je Asphaltschichtin . . . 23

3.4 Ermüdungsstatus aller berechneten Varianten; der Durchmesser gibt die Summe aller Einzelberechnungen an . . . . . . . . . . . . . . . . . . 24

3.5 Übersicht über die Standorte der Dauermesstellen zur Erfassung des Verkehrsaufkommens innerhalb der Bundesrepublik Deutschland . . . . . . . . 25

3.6 Relative Häufigkeit der Verkehrsbelastung in Abhängigkeit von der Tageszeit, Bundesdurchschnitt aller Messstationen, Zeitraum 2003 - 2016 . . . . . . . 26

3.7 Abweichung der Oberflächentemperaturen zum Monatsmittelwert in Abhängigkeit der Tageszeit, Periode 2020 bis 2049 für das Gebiet Dresden . . 27

3.8 Ermüdungsstatus in Abhängigkeit der Tageszeit und der Achslastklasse, Raum Dresden, (links) linearen Verteilung der Verkehrsstärken (rechts) zeitabhängigen Verteilung der Verkehrsstärken 


\section{Abbildungsverzeichnis}

3.9 Potenzialanalyse über den Einfluss einer Verkehrssteuerung für den Schwerverkehr . . . . . . . . . . . . . . . . . . . . . 29

4.1 Energiebilanz des thermischen Modells . . . . . . . . . . . . . . . . . . . 32

4.2 1D-Diskretisierung einer ebenen Platte mit der Finite-Volumen-Methode . . 33

4.3 Abweichung der Klimaparameter bezogen auf den über die Fläche des Stadtgebietes von Dresden gemittelten Durchschnittswert der Periode 1960 bis

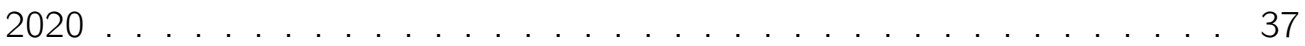

4.4 Wärmeleitfähigkeitsmessgerät ISOMET 2114 der Firma Applied Precision Ltd. 38

4.5 30-jähriger Maximalwert der Temperatur in Abhängigkeit von der Verkehrsfreigabe . . . . . . . . . . . . . . . . . . . . . 41

4.6 Abweichung der heißen Tage in der Asphaltbefestigung bezogen auf den Wert mit der Verkehrsfreigabe von 2020 . . . . . . . . . . . . . . 42

4.7 30-jähriger Mittelwert der Temperatur . . . . . . . . . . . . . . . . . . . . 44

4.8 Abweichung des Mittelwertes der Temperatur innerhalb der Asphaltdeckschicht bezogen auf den Basiswert mit der Verkehrsfreigabe von 2020 . . . 45

4.9 Mittlere Anzahl an Tagen mit Frost in der Asphaltbefestigung . . . . . . 47

4.10 Entwicklung der Anzahl an Frosttagen aller untersuchten Regionen . . . . . 48

4.11 Veränderung des Minimalwertes der Temperatur in der Asphalttragschicht aller untersuchten Regionen . . . . . . . . . . . . . . . . . . . . . . 49

4.12 Changepoint Analyse für unterschiedliche Parameter bezogen auf den Einfluss der thermischen Eigenschaften der Asphalttragschicht . . . . . . . . . . . 51

5.1 Beispieldaten mit vier Clusterzentren und zwei Merkmalen . . . . . . . . 55

5.2 Initiale Festlegung der Clusterzentren . . . . . . . . . . . . . . 55

5.3 Veränderung der Zuordnung der Einzelwerte zu den Clusterzentren in Abhängigkeit der Iterationsschritte des k-Means Algorithmus . . . . . . . . 56

5.4 Änderung der Abweichungen zwischen den Einzelwerten und den Clusterzentren in Abhängigkeit der Iterationsschritte . . . . . . . . . . . 57

5.5 Ergebnisdarstellung der Clusteranalyse mit variabler Anzahl an definierten Clustern . . . . . . . . . . . . . . . . . . . . . 58

5.6 Relevante Temperaturprofile nach den Analysen, Hintergrund entspricht dem ermittelten Temperaturspektrum und Farbe kennzeichnet die Häufigkeit des Auftretens, Betrachtungszeitraum 2020 - 2049, Stadtgebiet Dresden . . . 59

5.7 Darstellung der Funktionsverläufe sowie zufällig ausgewählter, jedoch zugehöriger Temperaturprofile, Betrachtungszeitraum 2020 - 2049 . . . . . . . 60

5.8 Abweichungen zwischen den Klassenmittelwerten (Temperaturprofilen) und allen zugehörigen Tempertaturbedingungen, 0,99 Quantil . . . . . . . . 62

5.9 Eingangsdaten und Ergebnisse einer Beispielrechnung zur Ermittlung des Einflusses der Temperatur auf die Dauerhaftigkeit von Straßenkonstruktionen; Parameterstudie mit hohen Abweichungen innerhalb der Asphalttragschicht 
5.10 Eingangsdaten und Ergebnisse einer Beispielrechnung zur Ermittlung des Einflusses der Temperatur auf die Dauerhaftigkeit von Straßenkonstruktionen; Parameterstudie mit hohen Abweichungen innerhalb der Asphaltdeckschicht 63

6.1 Relative Dauerhaftigkeit der Asphalttragschicht für das Gebiet Deutschland auf Basis der Mittelwertfunktionen; normiert auf den über die Fläche bestimmten Mittelwert aller Varianten mit der Verkehrsfreigabe ab 2020 .

6.2 Häufigkeitsverteilung der prognostizierten Dauerhaftigkeit für alle Landkreise normiert auf den Bundesdurchschnitt für die Periode ab dem Jahr 2020 . . 70

6.3 Ermüdungsstatus in Abhängigkeit des Bundeslandes und der Verkehrsfreigabe 71

6.4 Ermittelte Spurrinnentiefe für das Gebiet Deutschland auf Basis der Mittelwertfunktionen; normiert auf den Mittelwert aller Landkreise mit Verkehrsfreigabe ab $2020 \ldots \ldots$. . . . . . . . . . . . . . . . . 72

6.5 Häufigkeitsverteilung der Spurrinnentiefe für alle Landkreise normiert auf den Bundesdurchschnitt für die Periode ab dem Jahr 2020 . . . . . . . . . 74

6.6 Relative Spurrinnentiefe in Abhängigkeit der Verkehrsfreigabe, Basiswert ist der Einzelwert jeder Variante für das Jahr 2020 . . . . . . . . . . . . . . 74

7.1 Schematische Darstellung des Finite Elemente Modells zur Berechnung der mechanischen Beanspruchungen . . . . . . . . . . . . . . 78

7.2 Darstellung der Netzstruktur der ausgewählten mechanischen Modelle . . . 79

7.3 Verteilung der Temperaturen innerhalb der Asphaltschichten der Straßenkonstruktionen für den Simulationszeitraum 2011 bis 2040 . . . . . . . . . 80

7.4 Häufigkeitsverteilung der Cluster bzw. Temperaturprofile der Variante mit Rohrkollektor ... . . . . . . . . . . . . . . . . . . . . 81

7.5 Häufigkeitsverteilung der Cluster bzw. Temperaturprofile in Abhängigkeit der Tageszeit; Variante mit Rohrkollektor . . . . . . . . . . . . . . . 82

7.6 Verläufe der Clusterzentren . . . . . . . . . . . . . . . . . . . . . . . 82

7.7 Abweichung zwischen den repräsentativen Temperaturprofilen und den zugehörigen simulierten Temperaturbedingungen innerhalb der Straßenkonstruktion; Variante mit Rohrkollektor . . . . . . . . . . . . . . . . . . . .

7.8 Ermüdungsstatus in Abhängigkeit der Lage innerhalb der Straßenkonstruktion; Vergleich zwischen Referenzvariante und Variante mit integriertem Rohrkollektorsystem . . . . . . . . . . . . . . . . . . . . . .

7.9 Vergleich der relativen Änderungen der Nutzungsdauer zwischen der Referenzvariante und einem Straßenaufbau mit integriertem Kollektorsystem jeweils auf Basis der Mittelwertfunktionen oder normierten charakteristischen Temperaturprofilen; Referenzwert bildet der maximale Ermüdungsstatus der Referenzvariante auf Basis der Mittelwertfunktionen . . . . . . . . . . . . . 86

7.10 Oberflächentemperaturen der Simulationen auf Basis von Wetterdaten (Station Dresden Klotzsche) . . . . . . . . . . . . . . . . . . . 87

7.11 Anomalie der simulierten Oberflächentemperaturen, Referenzzeitraum 2013 bis $2017 \ldots \ldots \ldots$. . . . . . . . . . . . . . . . . . . . . . 88 
7.12 Häufigkeitsverteilung der Mittelwertfunktionen in Abhängigkeit der Tageszeit 89

7.13 Verlauf der Mittelwertfunktionen der Temperaturzustände für die Simulation mit Wetterdaten Station Dresden Klotzsche . . . . . . . . . . . . . . . 89

7.14 Relative Änderung der Nutzungszeit, Referenzzeitraum 2013 bis 2017 . . . 90

7.15 Versuchsaufbau zur Untersuchung der Asphalt-PCM-Platte . . . . . . . . . 93

7.16 Temperatur innerhalb der Asphaltproben und der Temperierkammer in Abhängigkeit der Dauer des Experimentes . . . . . . . . . . . . . . . . . . . 94

7.17 Temperaturverlauf der einzelnen Variante mit unterschiedlichen Schmelzpunkten im Vergleich zur Referenzvariante, Ausschnitt . . . . . . . . . . . 95

7.18 Häufigkeitsverteilung der Kerntemperaturen der Referenzvariante und der Varianten mit PCM . . . . . . . . . . . . . . . . . . . . . . . . 96

7.19 Akkumulierte stündliche Abweichung der Temperaturen auf der Oberseite einer Straßenkonstruktion in Abhängigkeit von der Tageszeit . . . . . . . . 97

7.20 Relative Abweichung des Ermüdungsstatus zwischen den PCM-Varianten und der Referenzvariante unter Berücksichtigung der Tagesganglinie des Verkehrs . . . . . . . . . . . . . . . . . . . . . . . . 98 


\section{Tabellenverzeichnis}

3.1 Eingangsdaten der multikriteriellen Analyse . . . . . . . . . . . . . 21

3.2 Ermüdungsfunktionen für die Nachweisführung . . . . . . . . . . . . . . . 23

4.1 Thermophysikalische Materialparameter der verwendeten Asphalte . . . . . 38

6.1 Eingangswerte für die Dimensionierungsrechnungen . . . . . . . . . . . . 67

6.2 Parameterdarstellung der Materialkennwerte der Basisvariante . . . . . . . 67

B.1 Relative Häufigkeitsverteilung der Fahrzeuge in Abhängigkeit der Tageszeit 186 

Anhang 



\section{A Klimarisiken}

\section{A.1 Change Point Analyse}

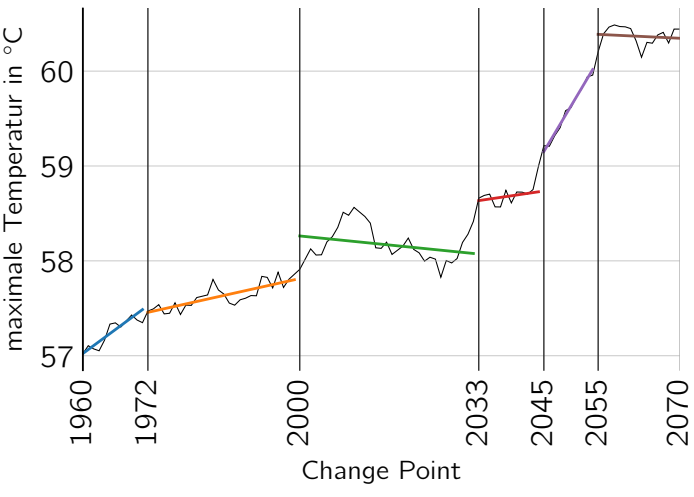

(a) maximale Temperatur

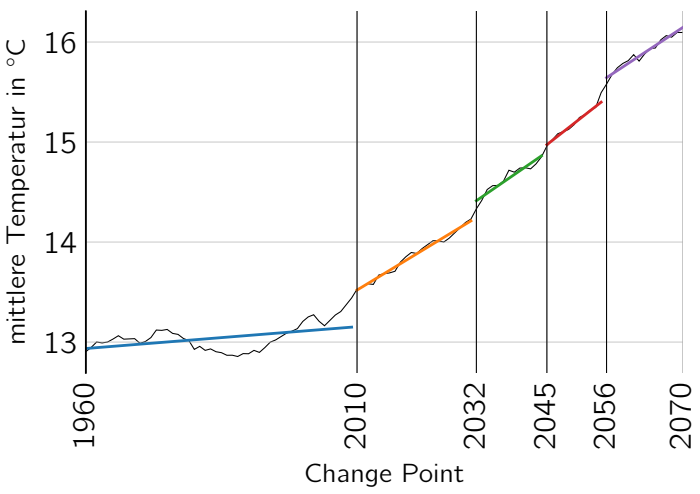

(c) mittlere Temperatur

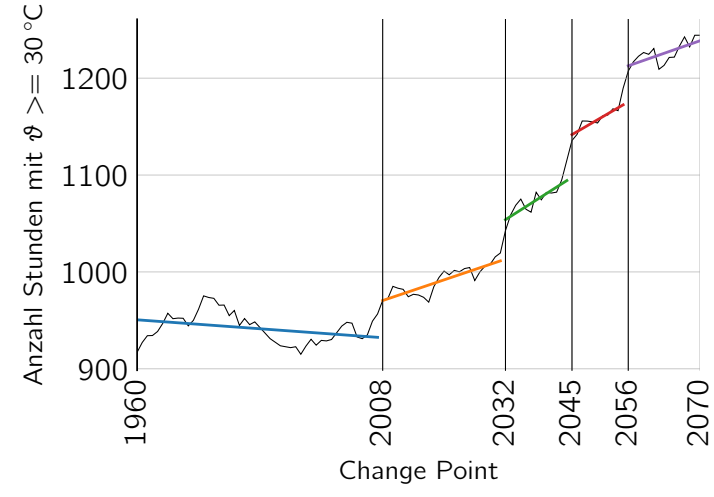

(b) Anzahl an Stunden mit einer Temperatur größer gleich $30^{\circ} \mathrm{C}$

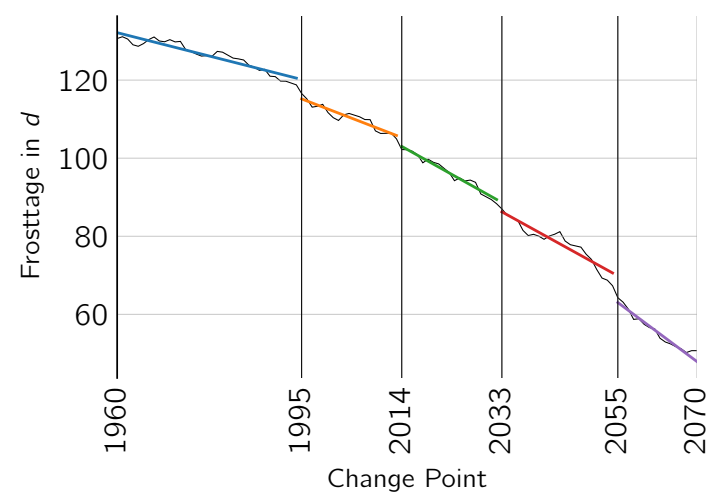

(d) Anzahl an Frosttage

\section{Asphaltdeckschicht}


Anhang

A.2 Klimaparameter

A.2.1 Asphaltdeckschicht 
A Klimarisiken

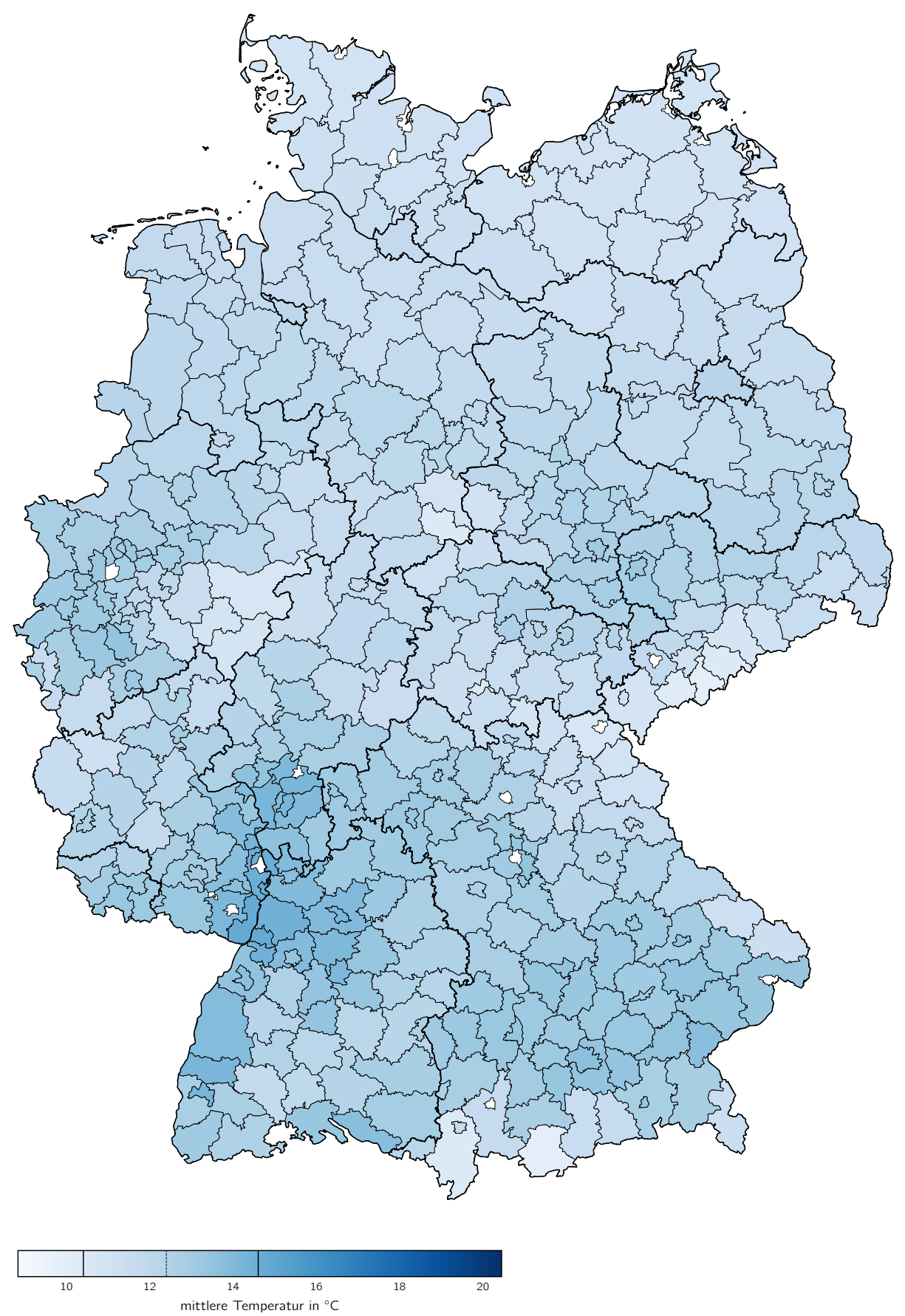

Mittlere Temperatur innerhalb der Asphaltdeckschicht, Verkehrsfreigabe 1960 


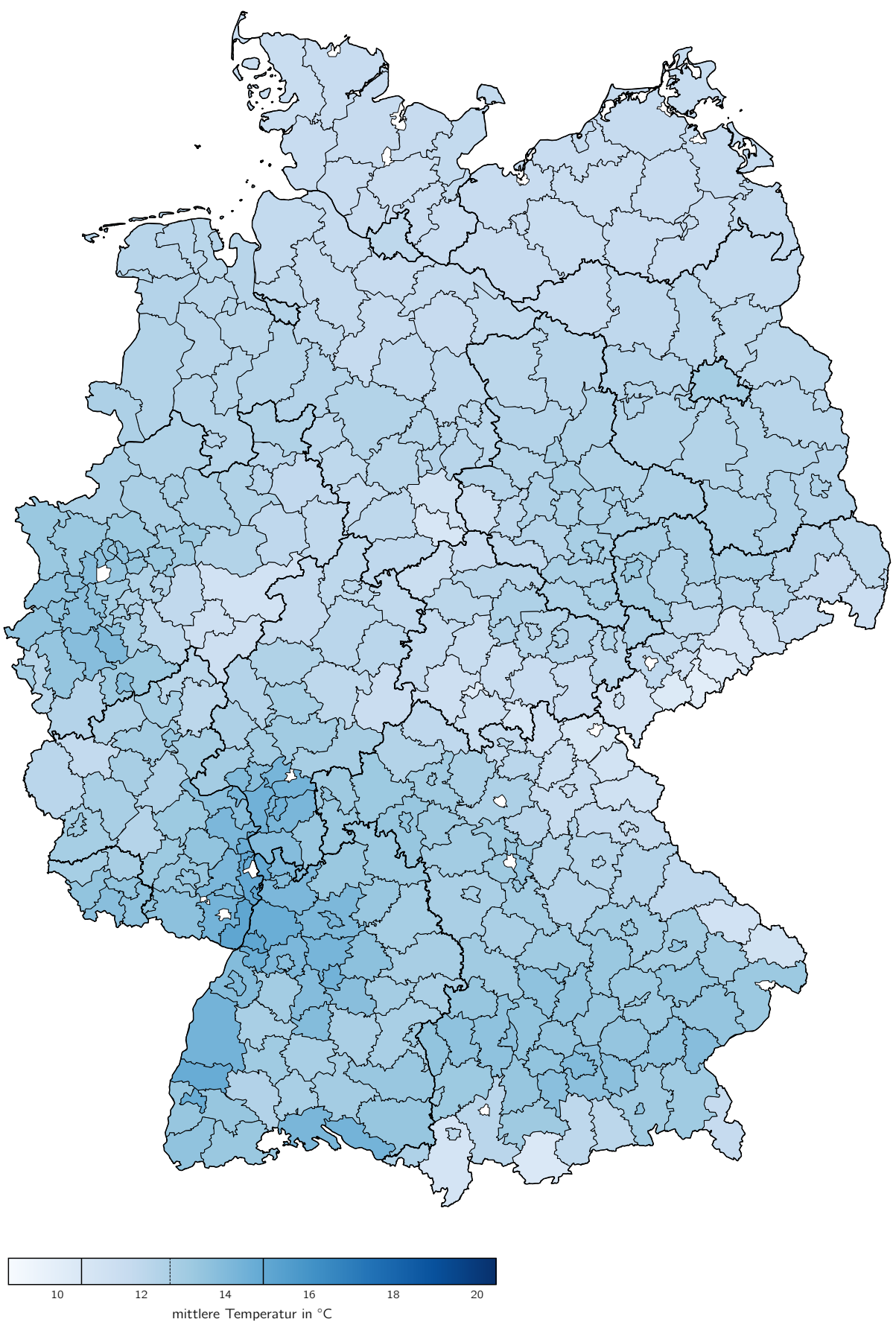

Mittlere Temperatur innerhalb der Asphaltdeckschicht, Verkehrsfreigabe 2020 
A Klimarisiken

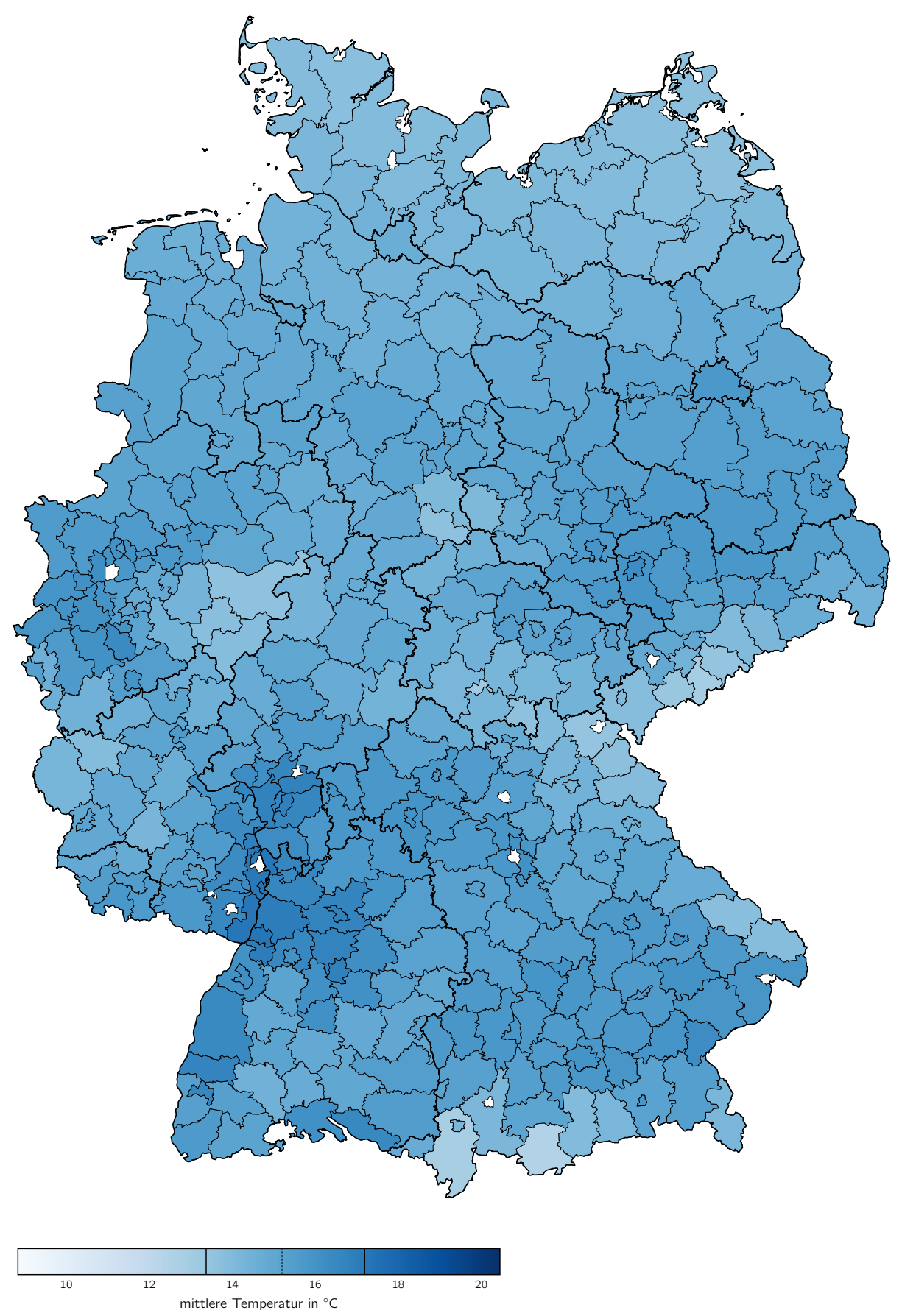

Mittlere Temperatur innerhalb der Asphaltdeckschicht, Verkehrsfreigabe 2040 


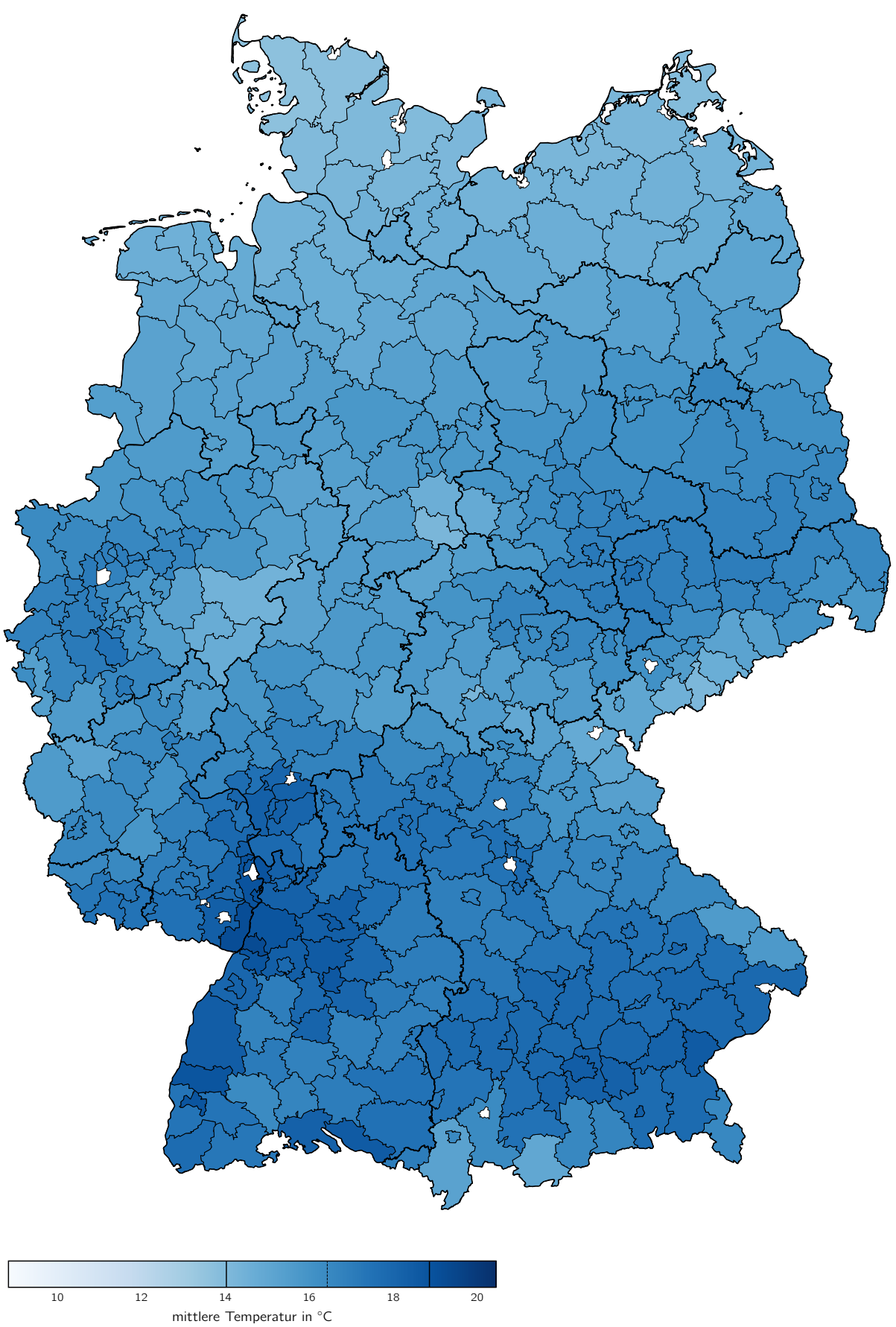

Mittlere Temperatur innerhalb der Asphaltdeckschicht, Verkehrsfreigabe 2070 
A Klimarisiken

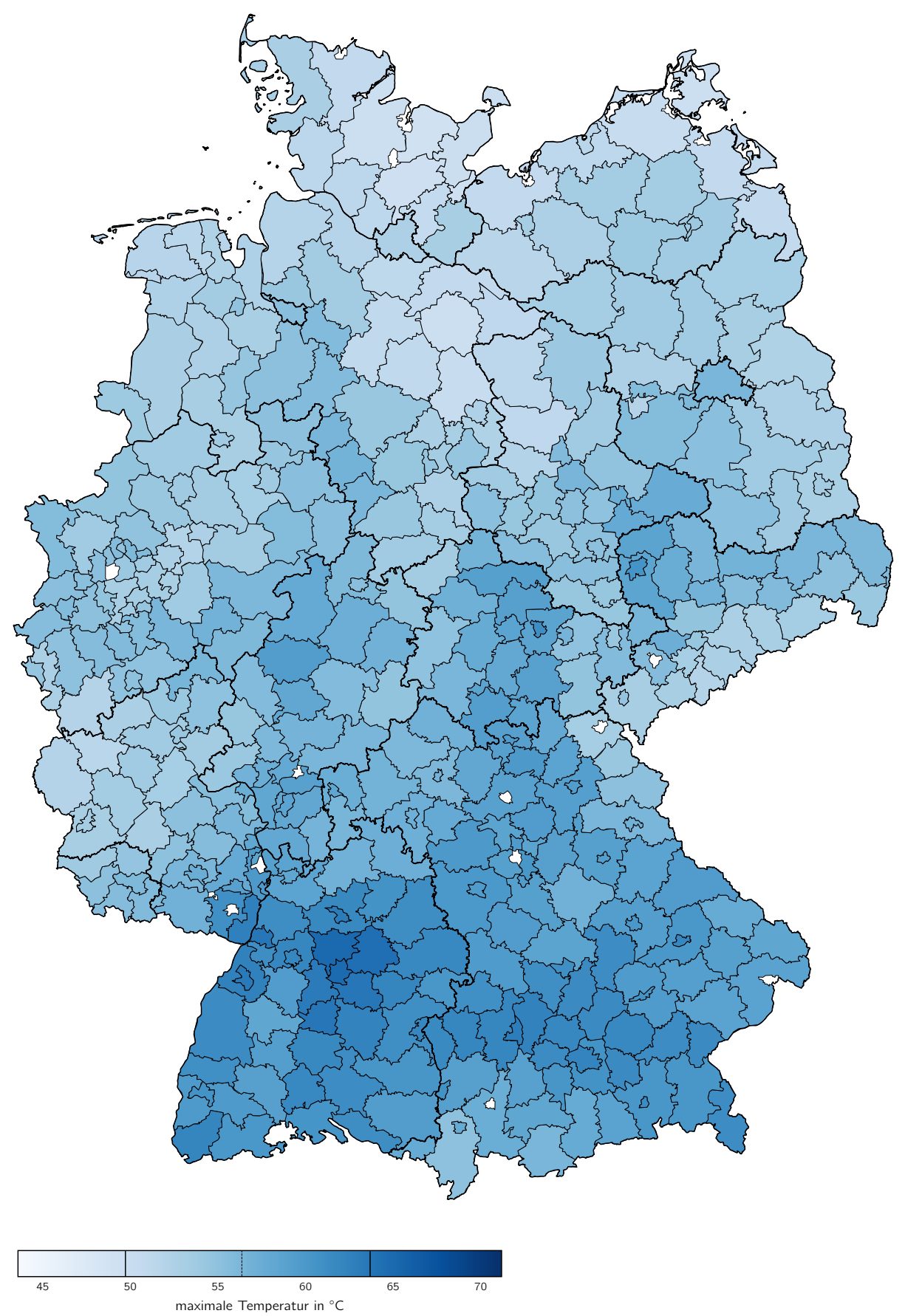

Maximale Temperaturen innerhalb der Asphaltdeckschicht, Verkehrsfreigabe 1960 


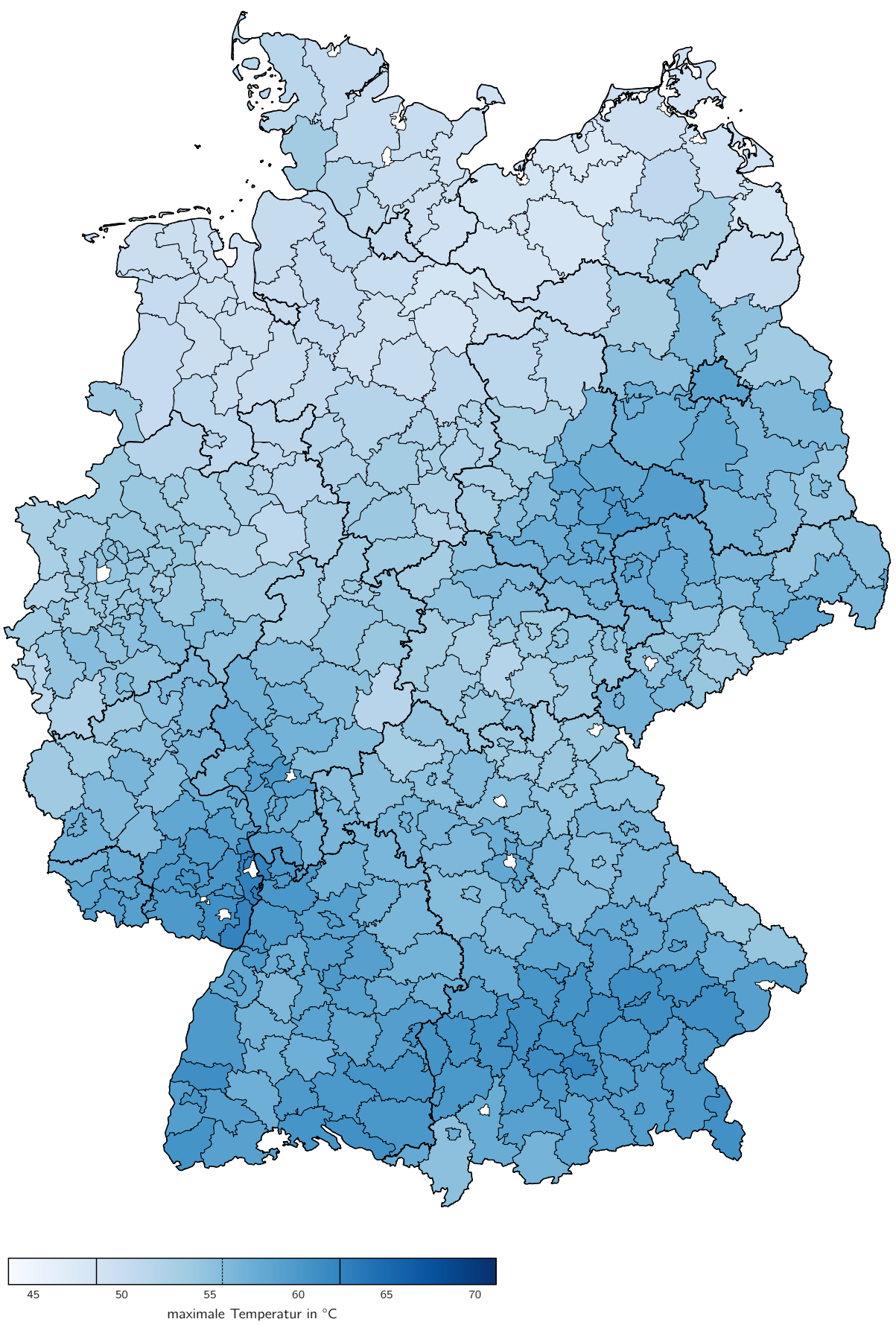

Maximale Temperaturen innerhalb der Asphaltdeckschicht, Verkehrsfreigabe 2020 
A Klimarisiken

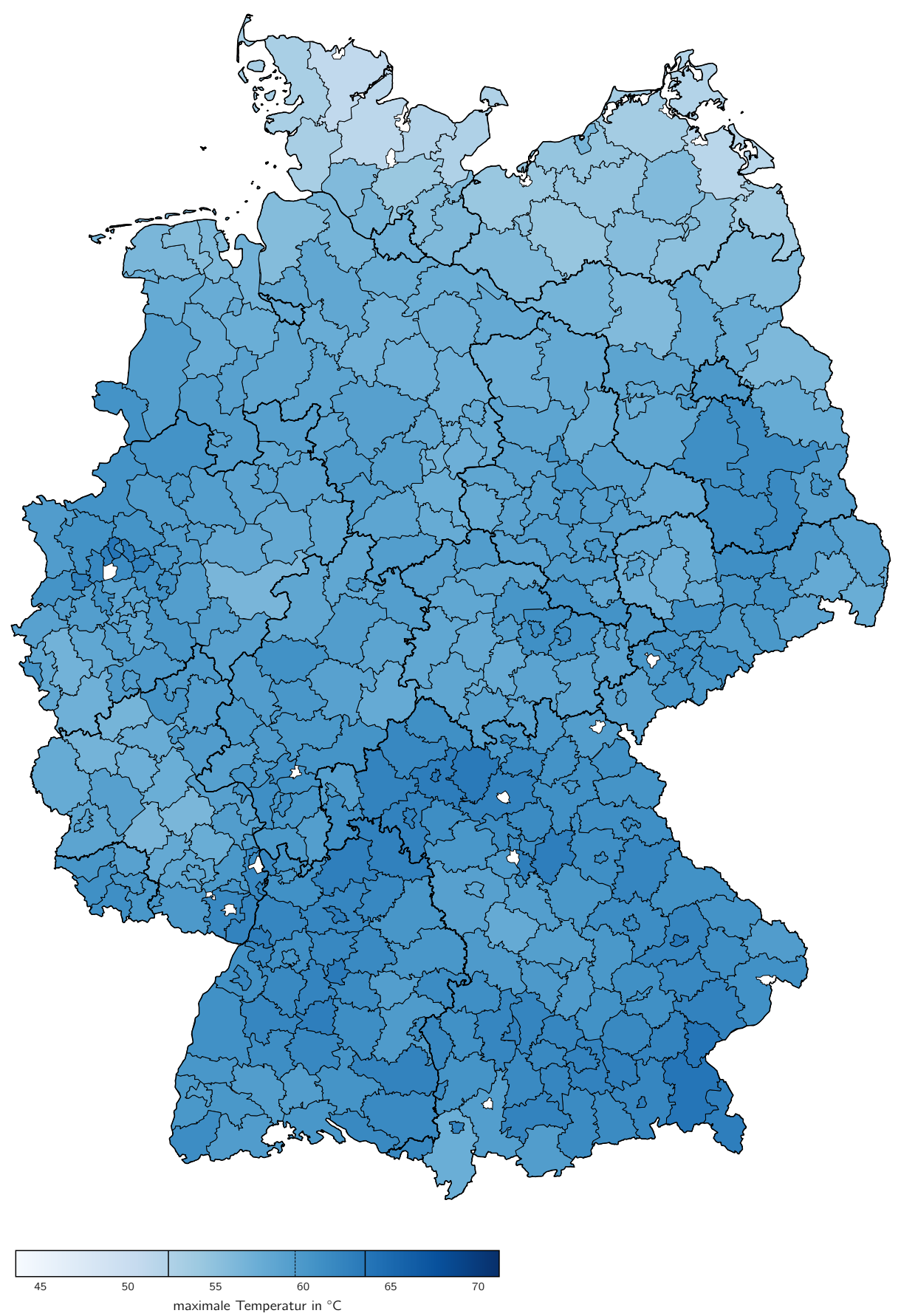

Maximale Temperaturen innerhalb der Asphaltdeckschicht, Verkehrsfreigabe 2040 


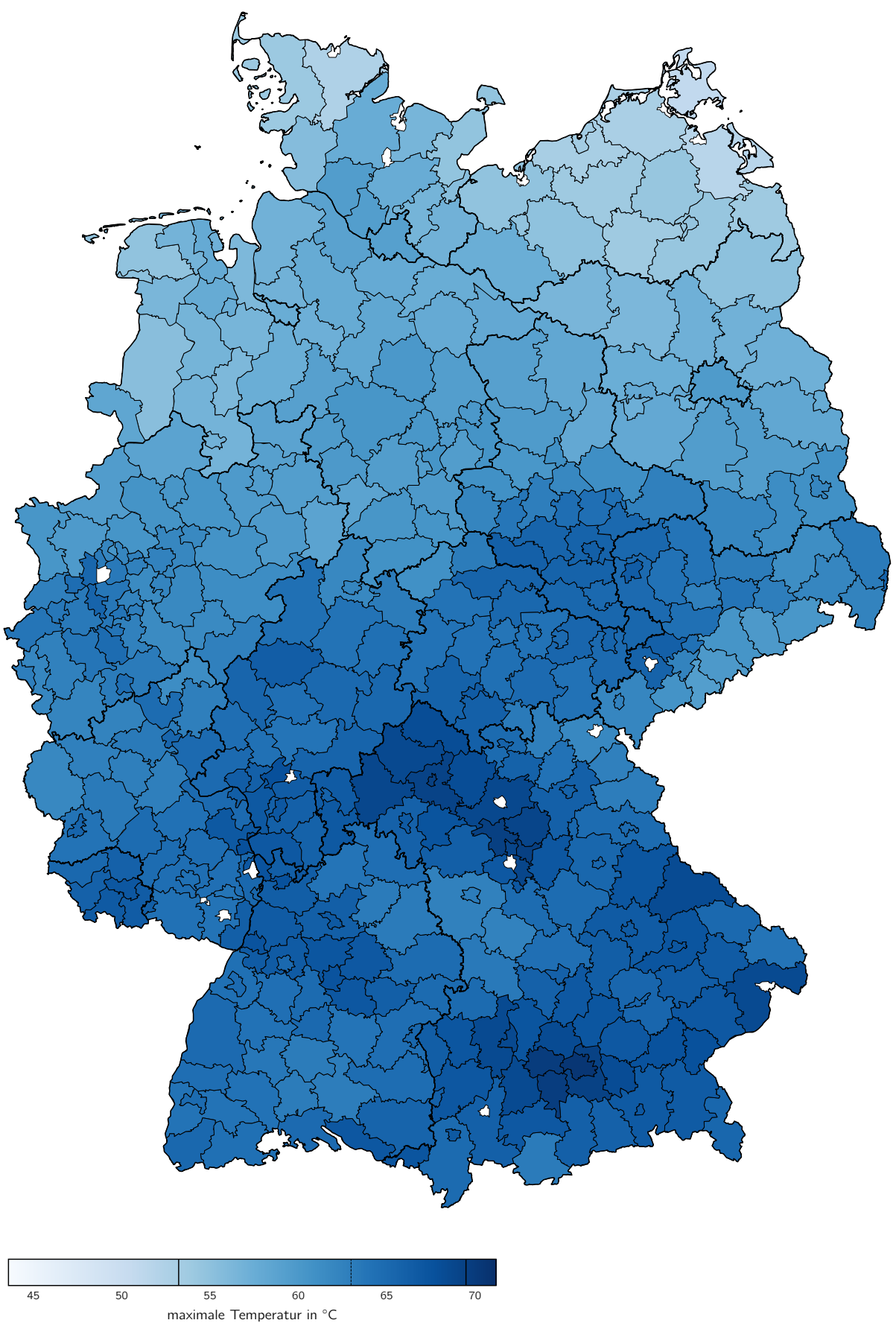

Maximale Temperaturen innerhalb der Asphaltdeckschicht, Verkehrsfreigabe 2070 
A Klimarisiken

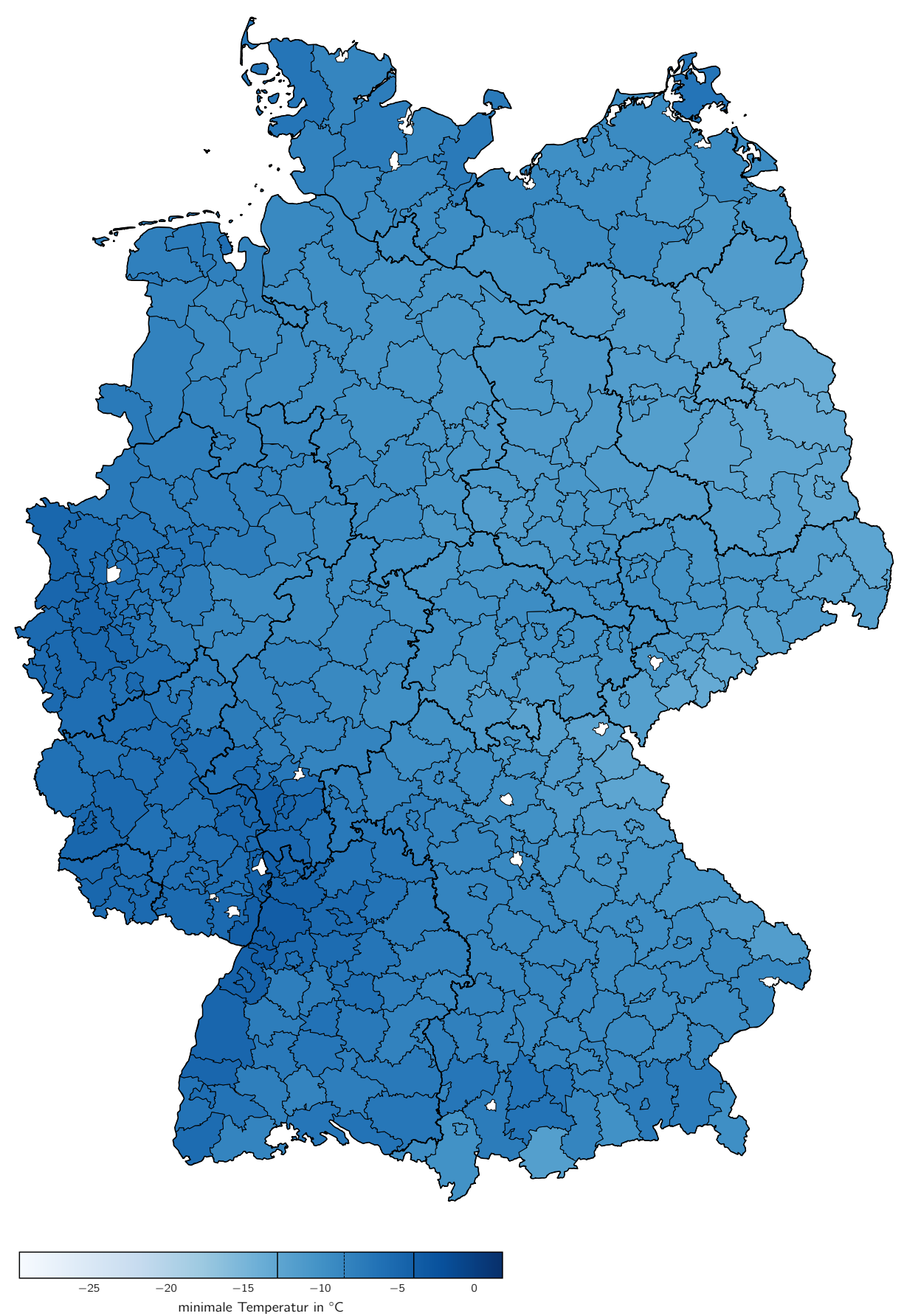

Minimale Temperaturen innerhalb der Asphaltdeckschicht, Verkehrsfreigabe 1960 


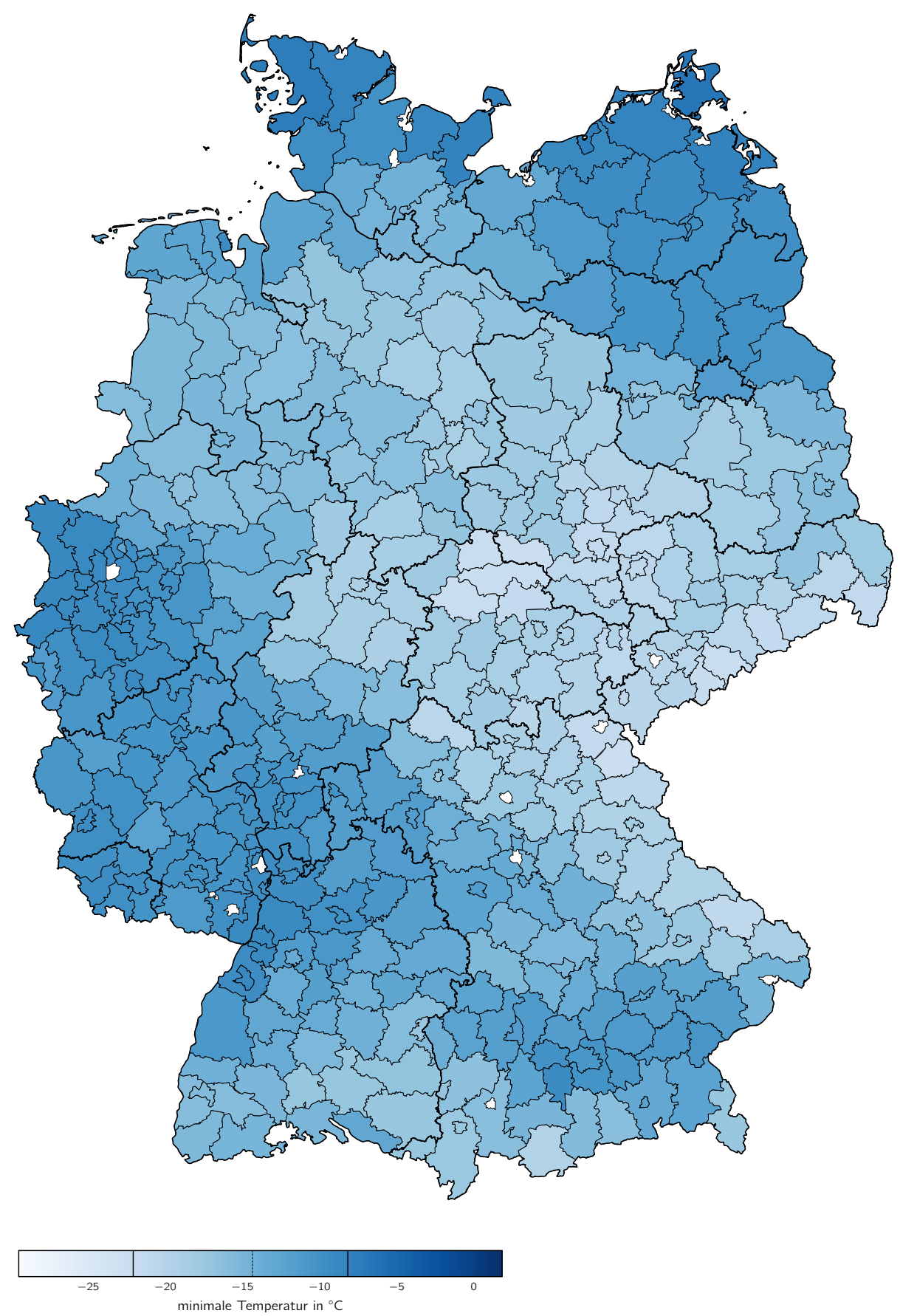

Minimale Temperaturen innerhalb der Asphaltdeckschicht, Verkehrsfreigabe 2020 
A Klimarisiken

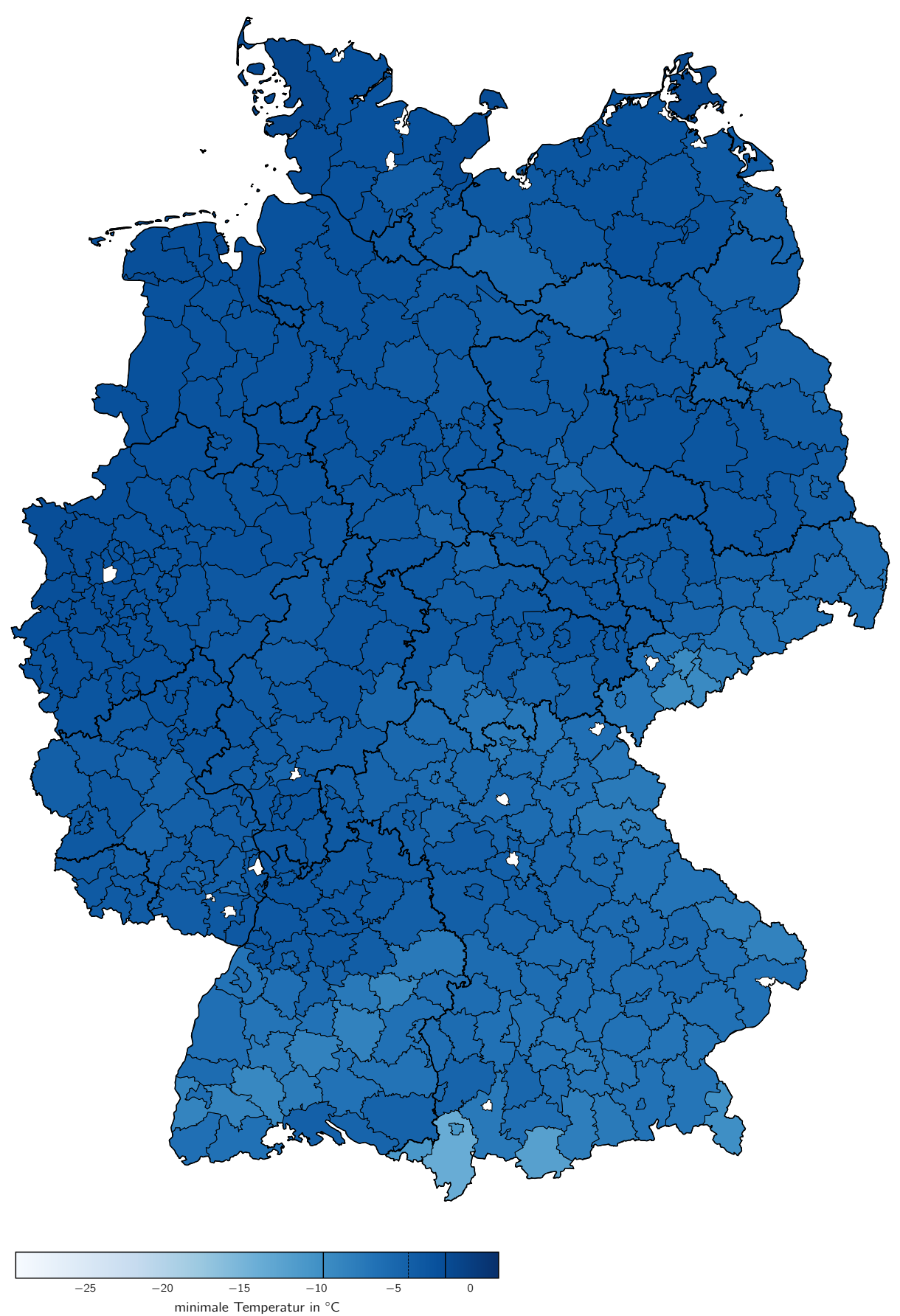

Minimale Temperaturen innerhalb der Asphaltdeckschicht, Verkehrsfreigabe 2040 


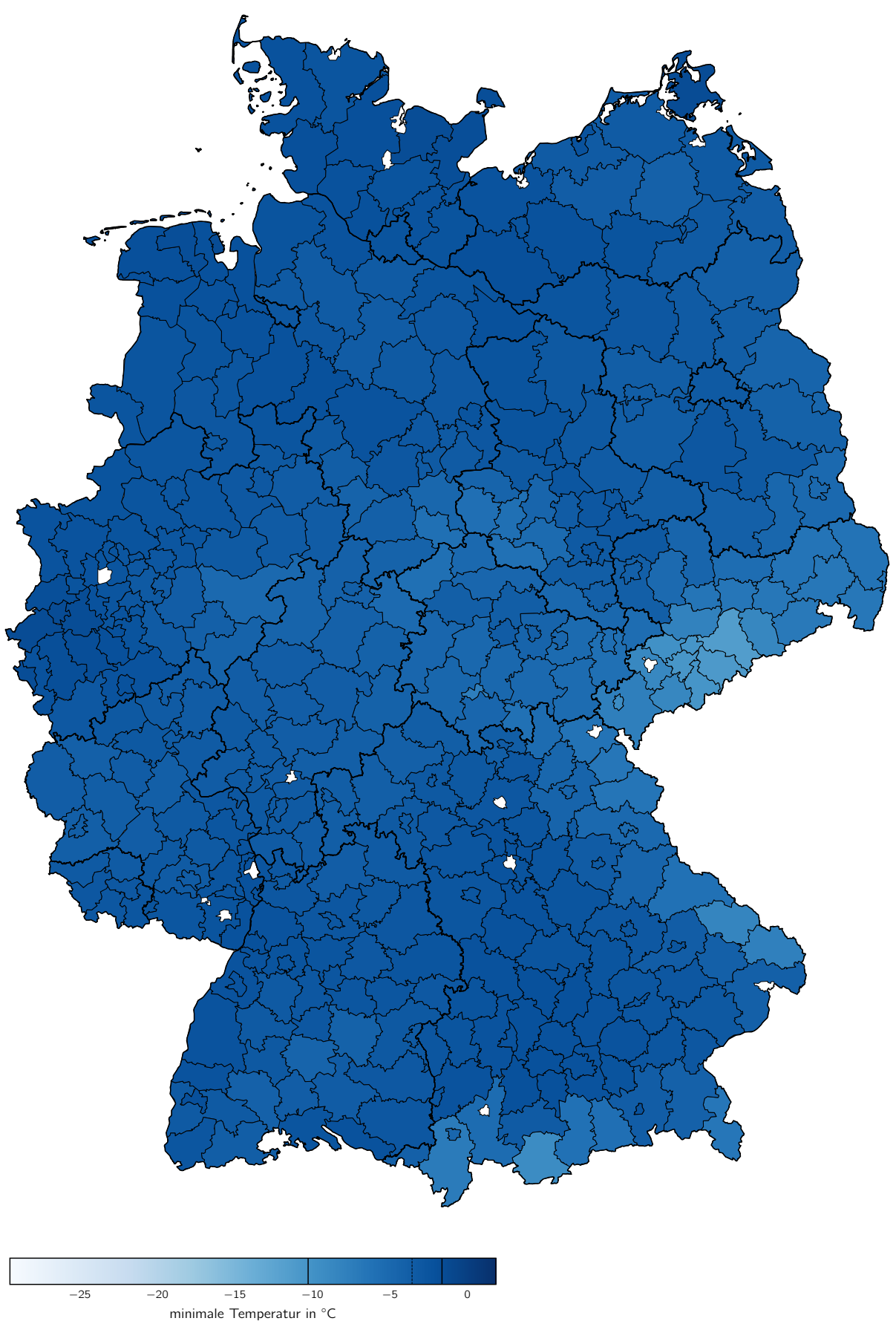

Minimale Temperaturen innerhalb der Asphaltdeckschicht, Verkehrsfreigabe 2070 
A Klimarisiken

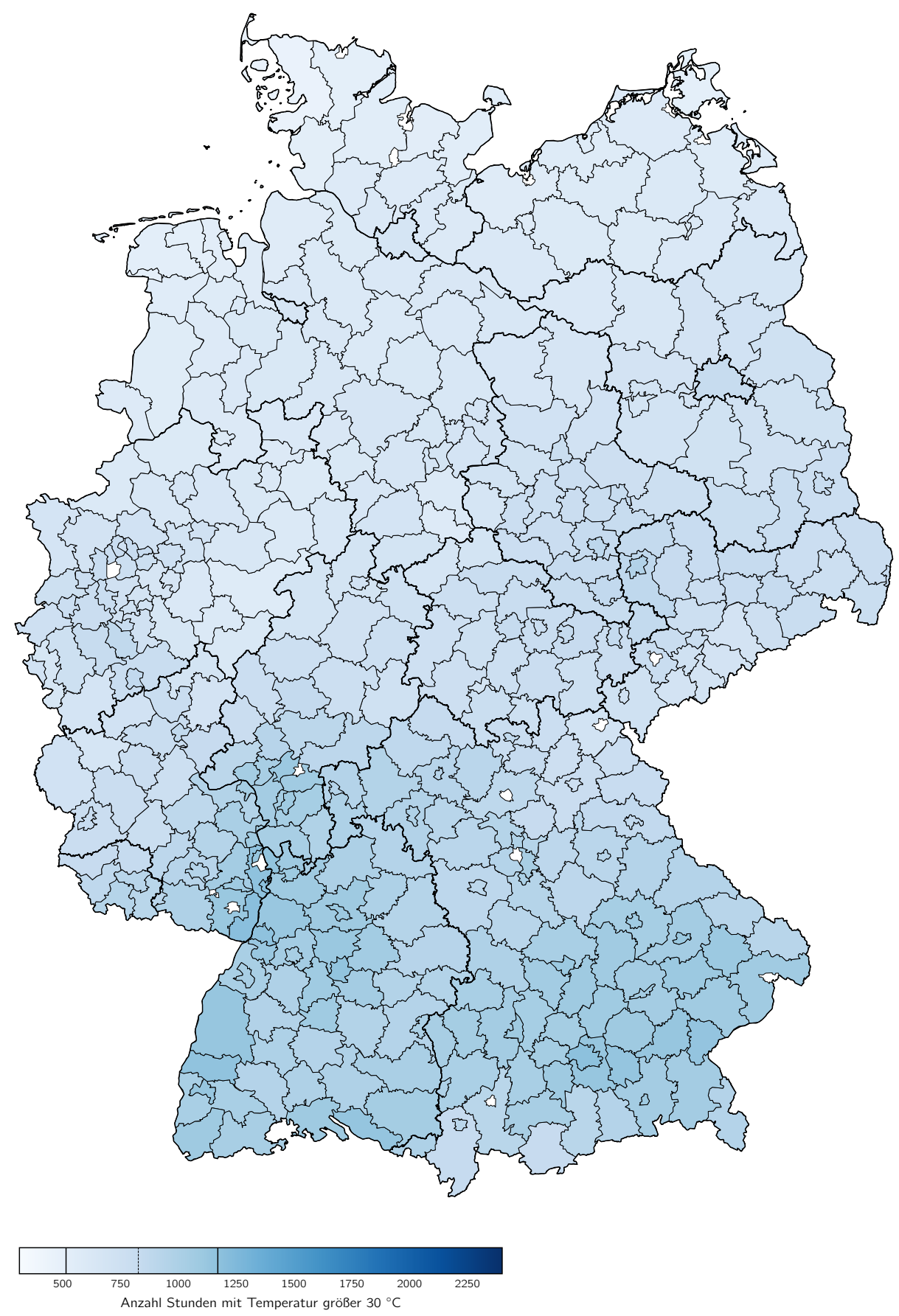

Gesamtzahl an Stunden in der die Temperatur innerhalb der Asphaltdeckschicht größer als 30 ${ }^{\circ} \mathrm{C}$ vorherrscht, Verkehrsfreigabe 1960 


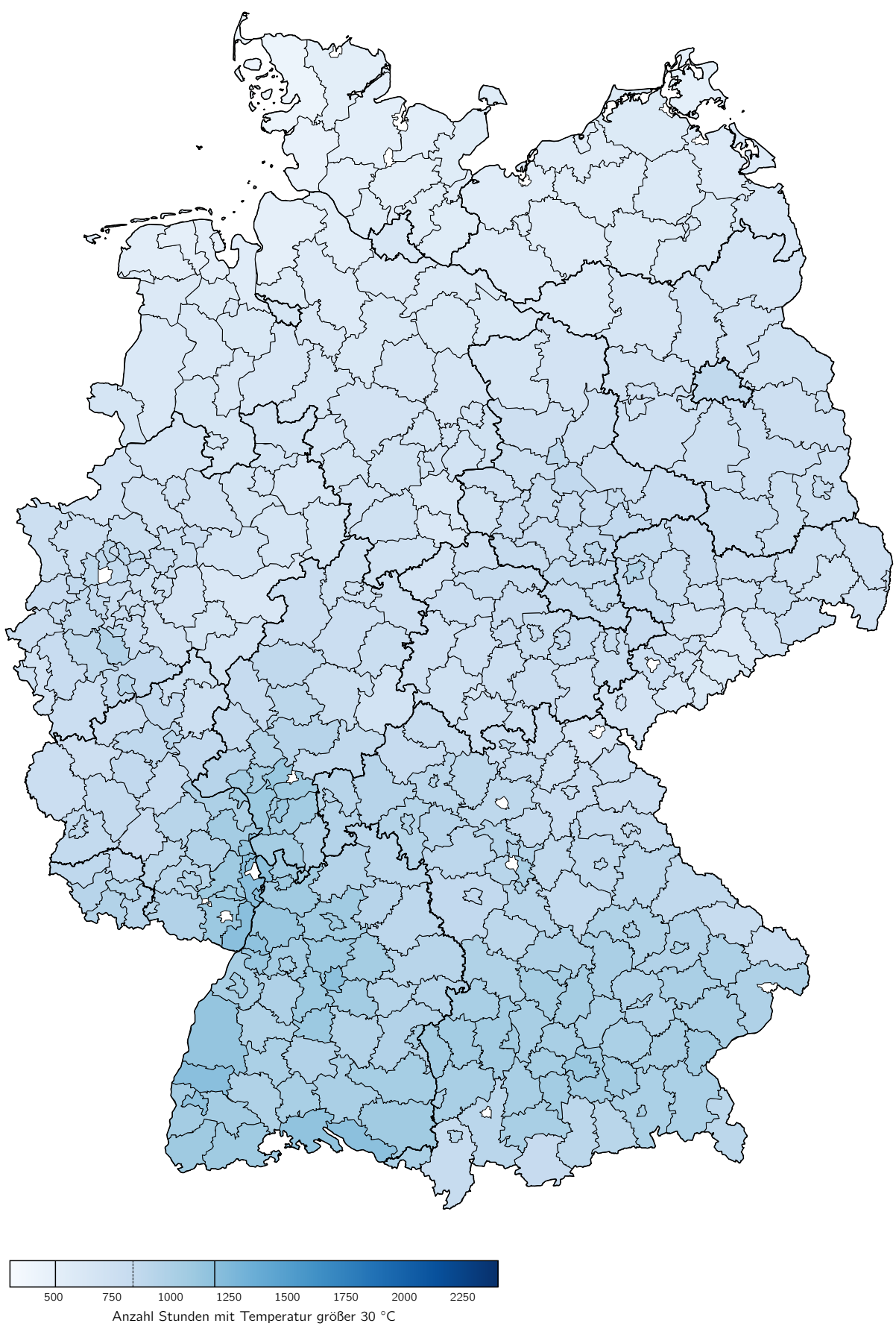

Gesamtzahl an Stunden in der die Temperatur innerhalb der Asphaltdeckschicht größer als 30 ${ }^{\circ} \mathrm{C}$ vorherrscht, Verkehrsfreigabe 2020 
A Klimarisiken

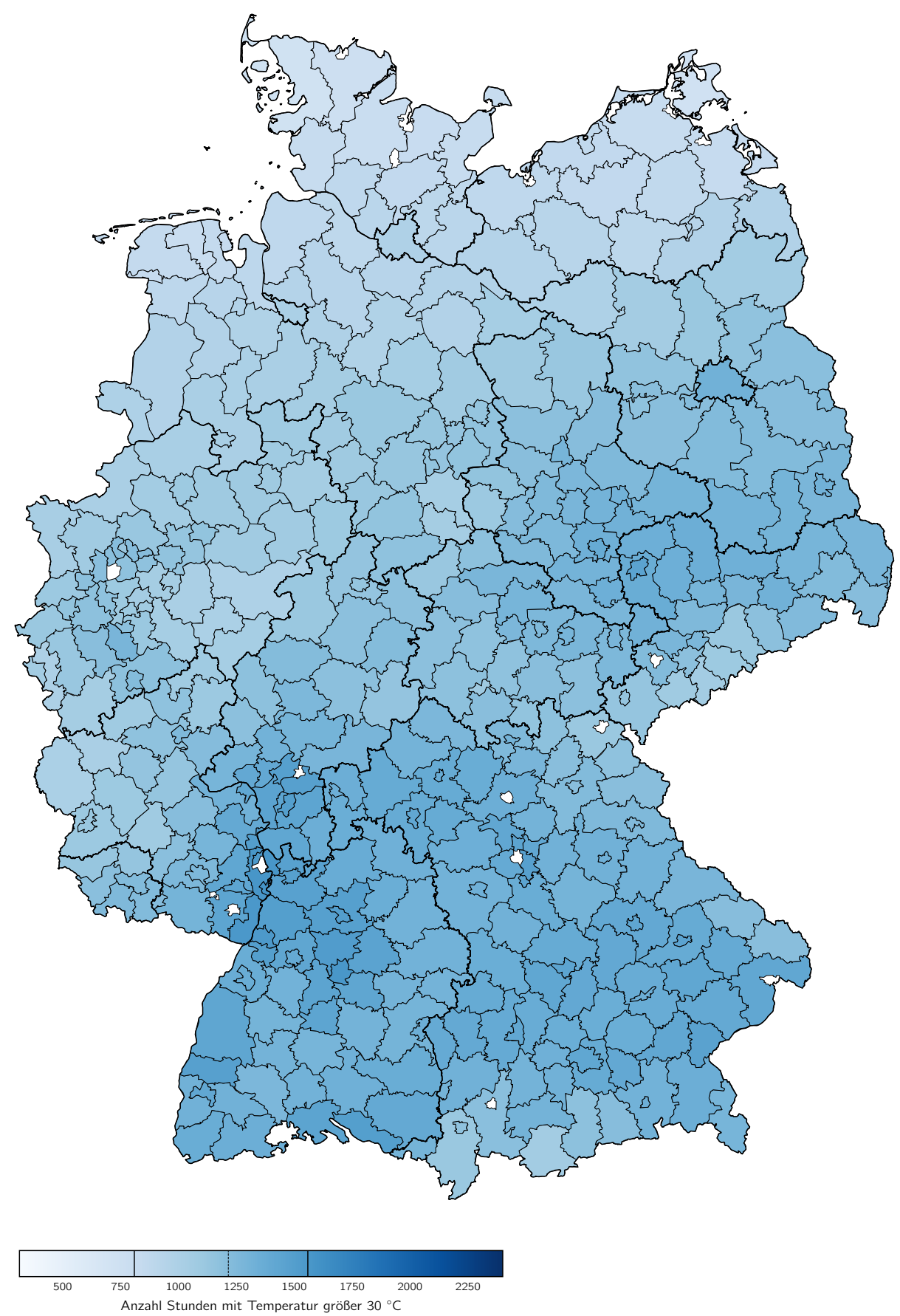

Gesamtzahl an Stunden in der die Temperatur innerhalb der Asphaltdeckschicht größer als 30 ${ }^{\circ} \mathrm{C}$ vorherrscht, Verkehrsfreigabe 2040 


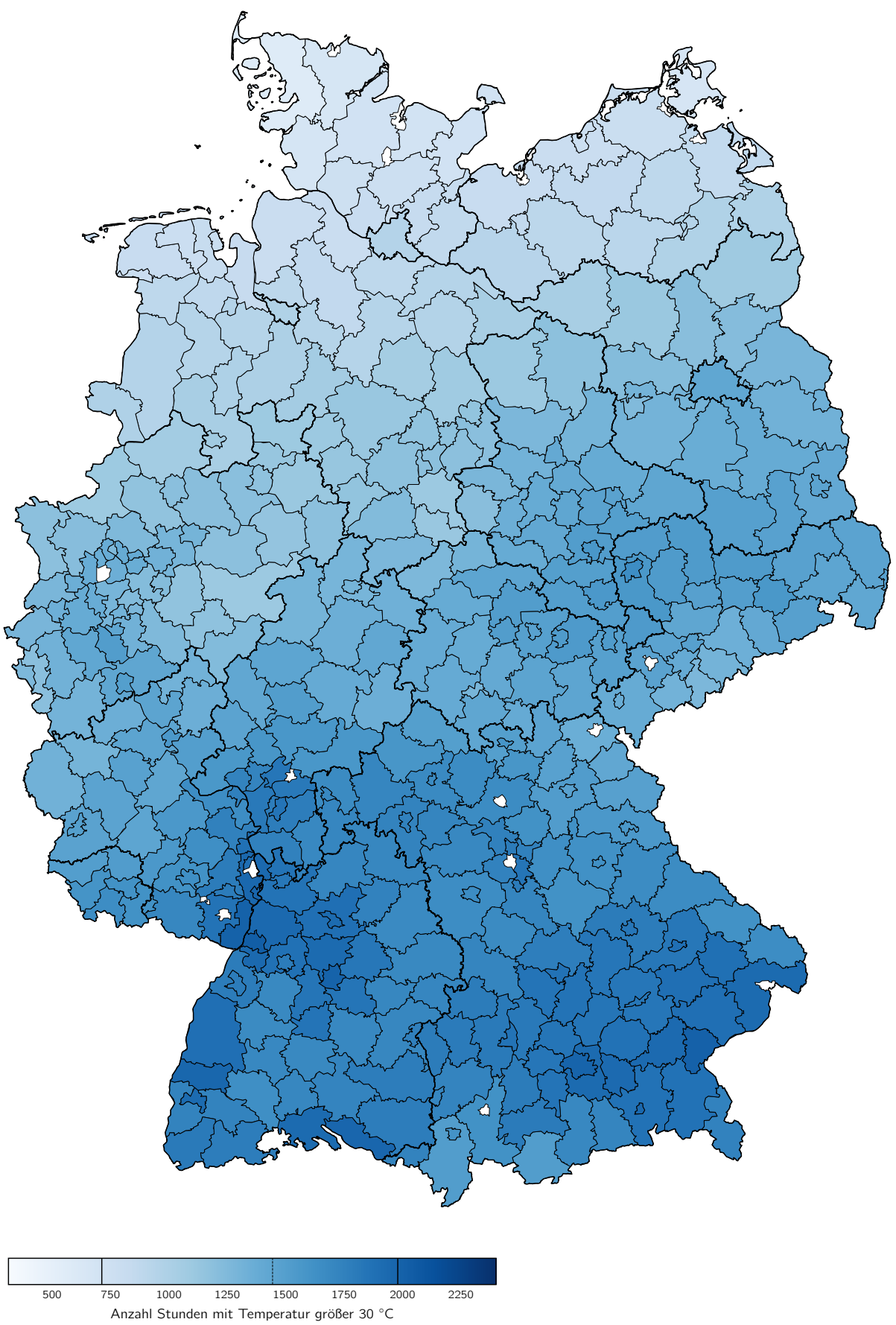

Gesamtzahl an Stunden in der die Temperatur innerhalb der Asphaltdeckschicht größer als 30 ${ }^{\circ} \mathrm{C}$ vorherrscht, Verkehrsfreigabe 2070 


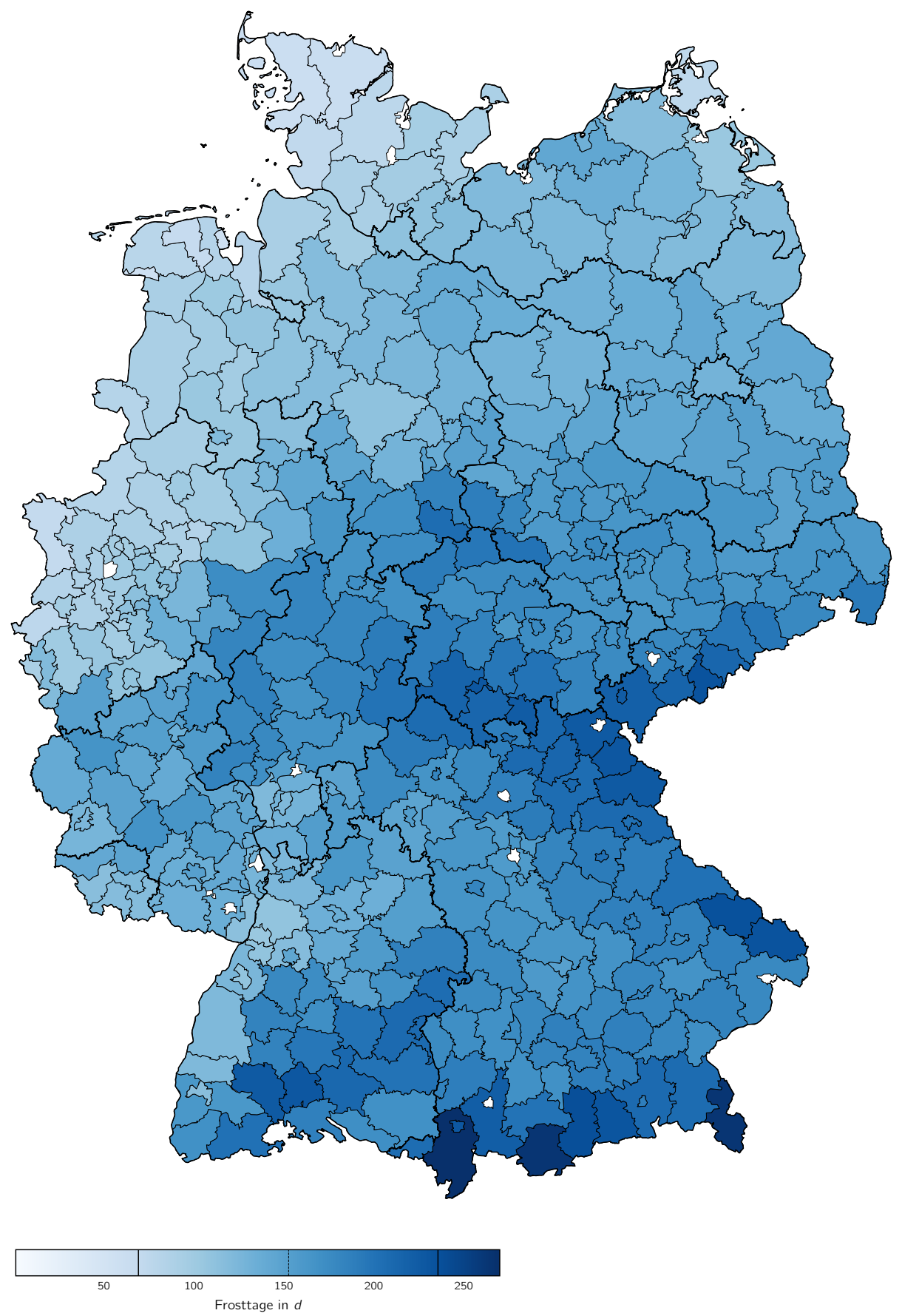

Frosttage, Asphaltdeckschicht, Verkehrsfreigabe 1960 


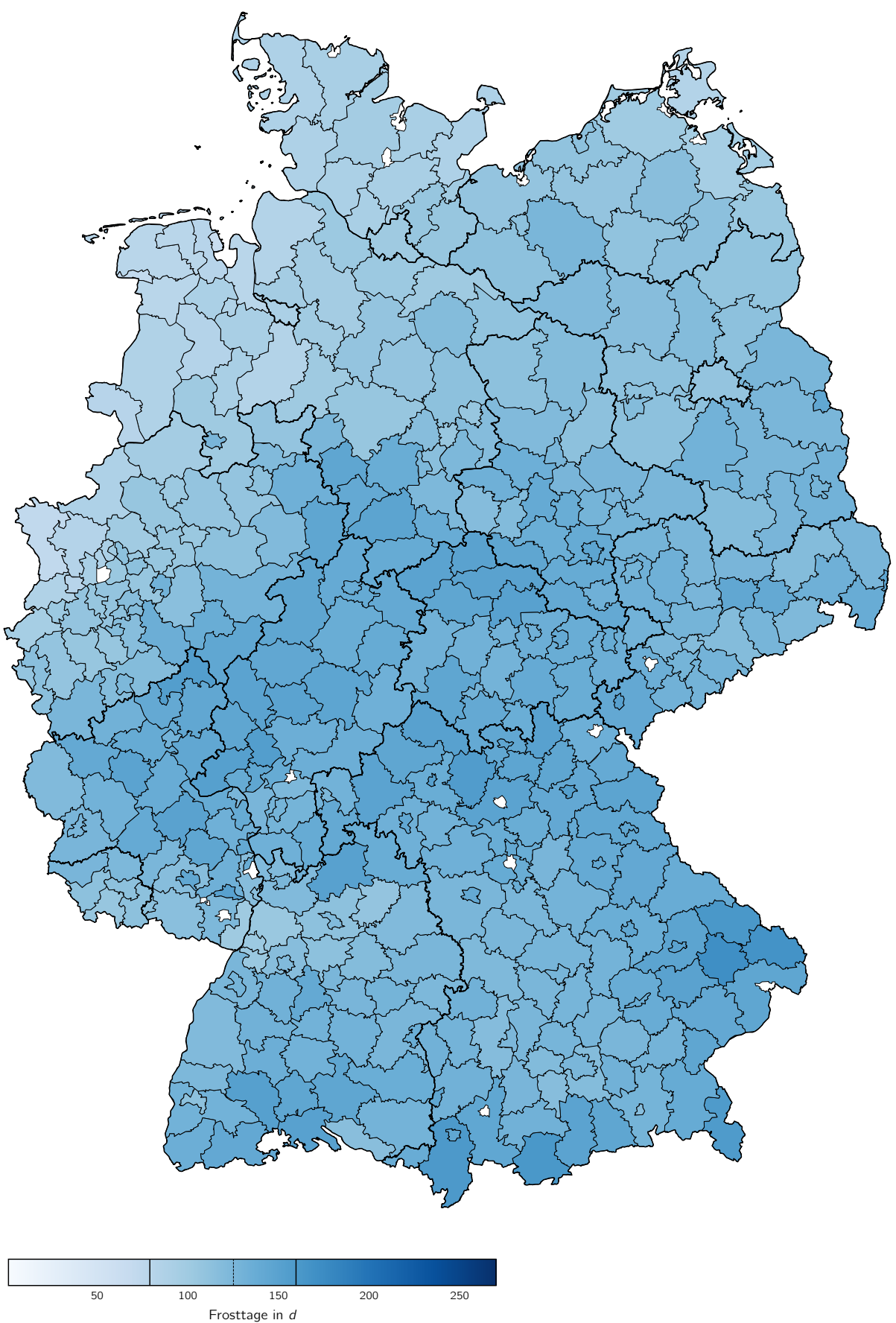

Frosttage, Asphaltdeckschicht, Verkehrsfreigabe 2020 
A Klimarisiken

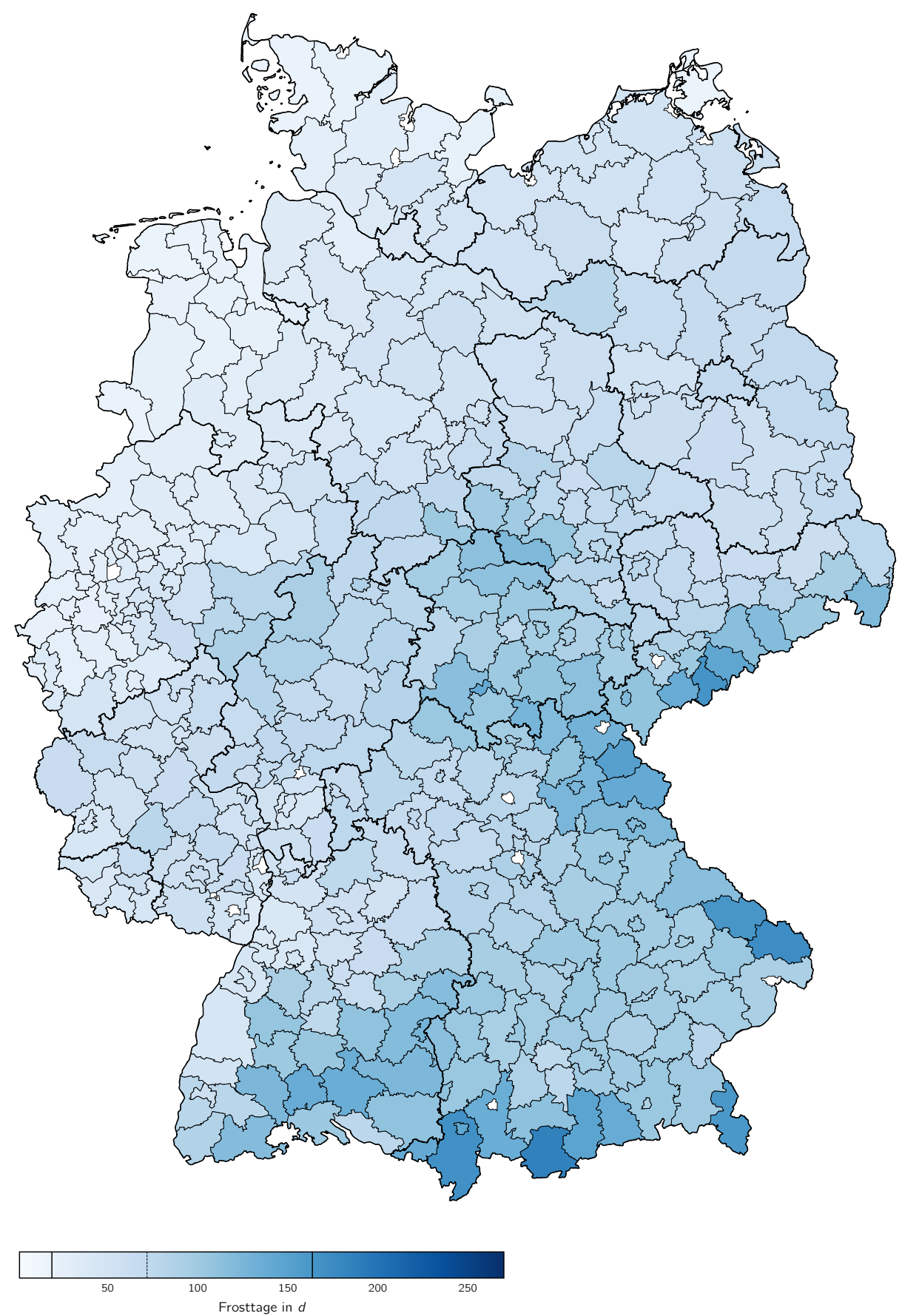

Frosttage, Asphaltdeckschicht, Verkehrsfreigabe 2040 


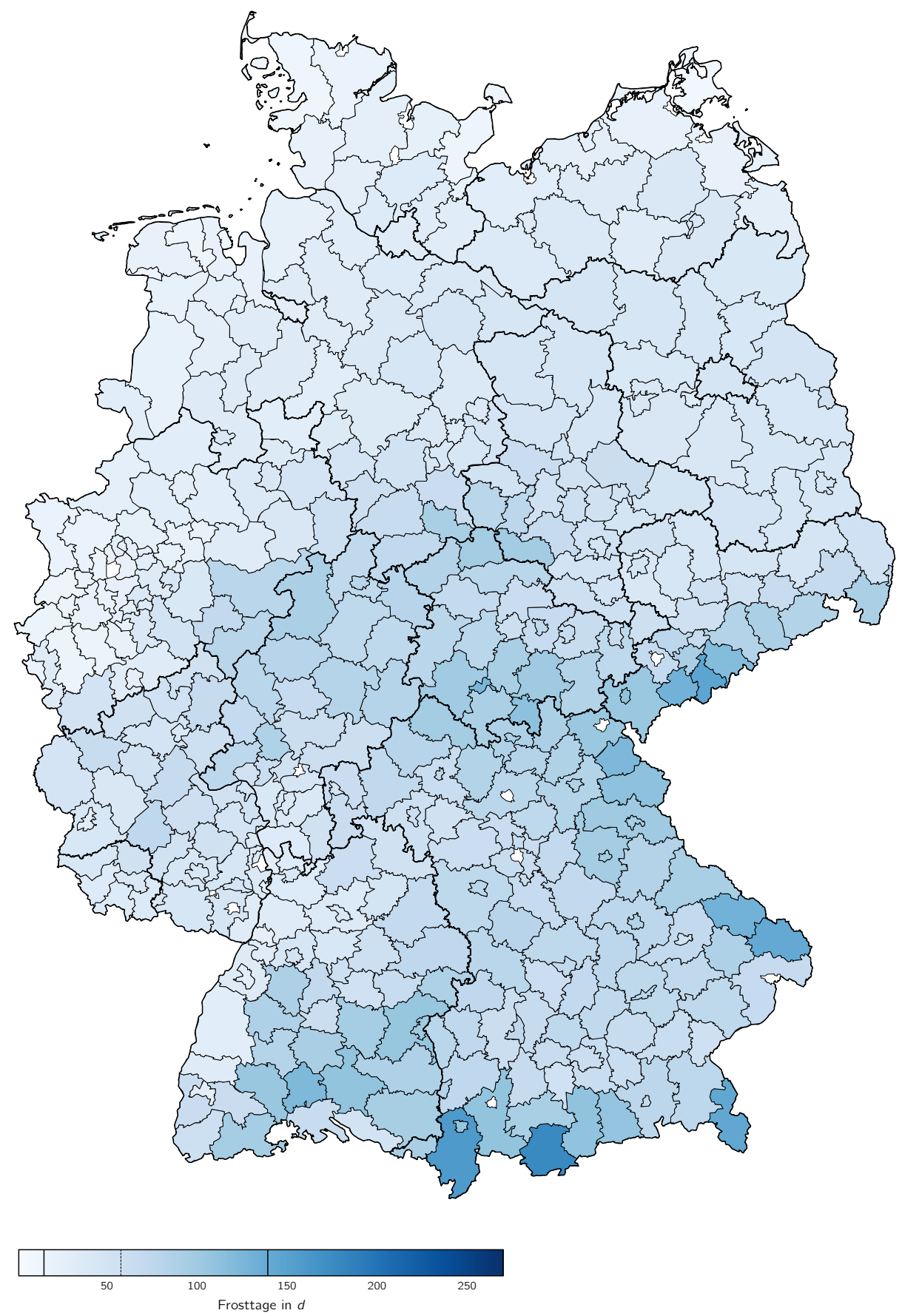

Frosttage, Asphaltdeckschicht, Verkehrsfreigabe 2070 


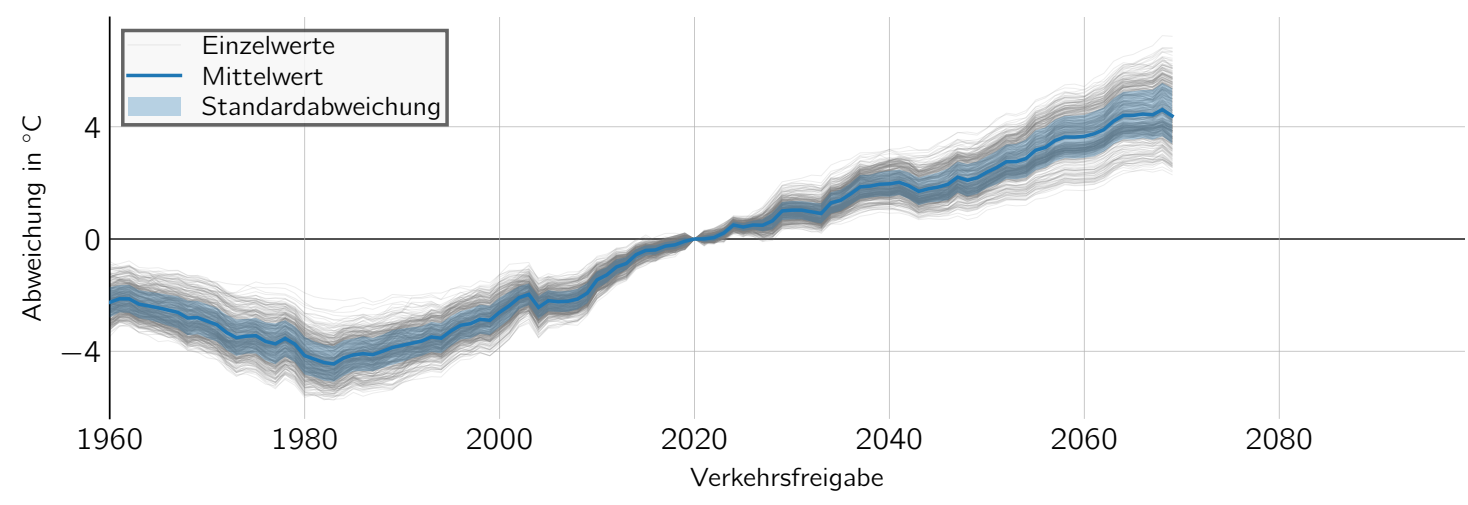

Abweichung der minimalen Temperatur innerhalb der Asphaltdeckschicht bezogen auf den Basiswert mit der Verkehrsfreigabe von 2020

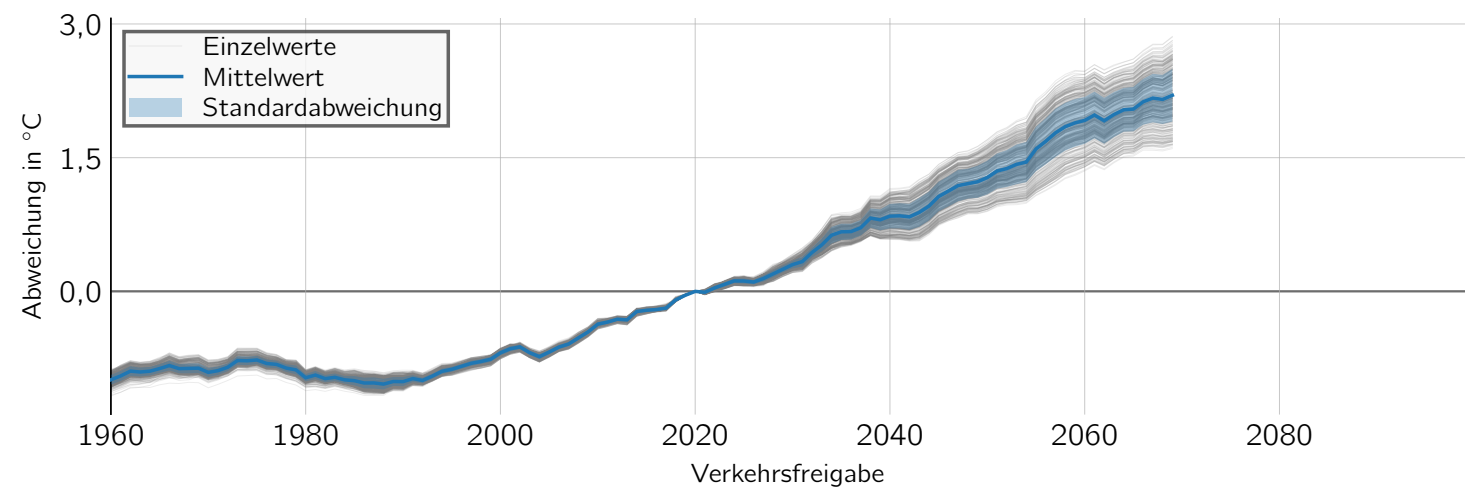

Abweichung der mittleren Temperatur innerhalb der Asphaltdeckschicht bezogen auf den Basiswert mit der Verkehrsfreigabe von 2020

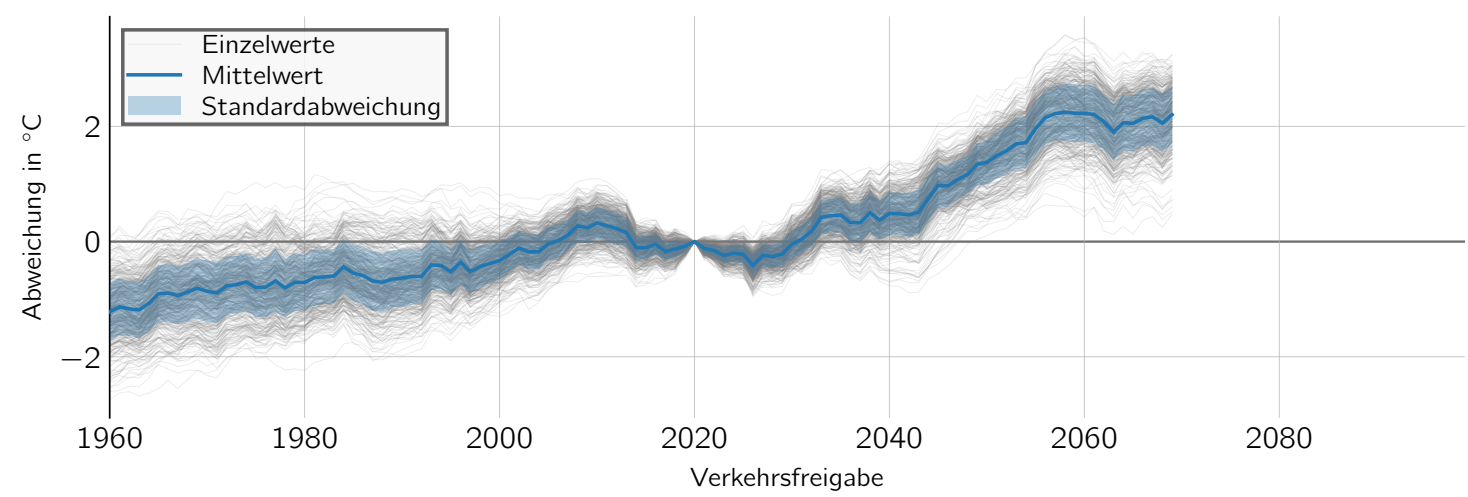

Abweichung der maximalen Temperatur innerhalb der Asphaltdeckschicht bezogen auf den Basiswert mit der Verkehrsfreigabe von 2020 


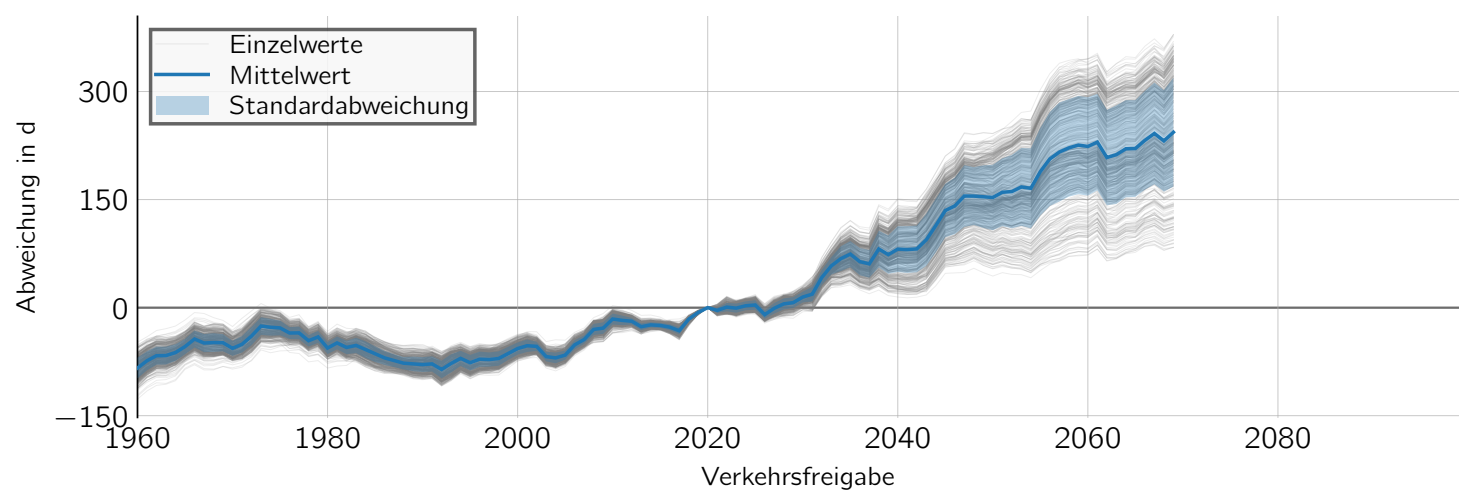

Abweichung der maximalen Temperatur innerhalb der Asphaltdeckschicht bezogen auf den Basiswert mit der Verkehrsfreigabe von 2020

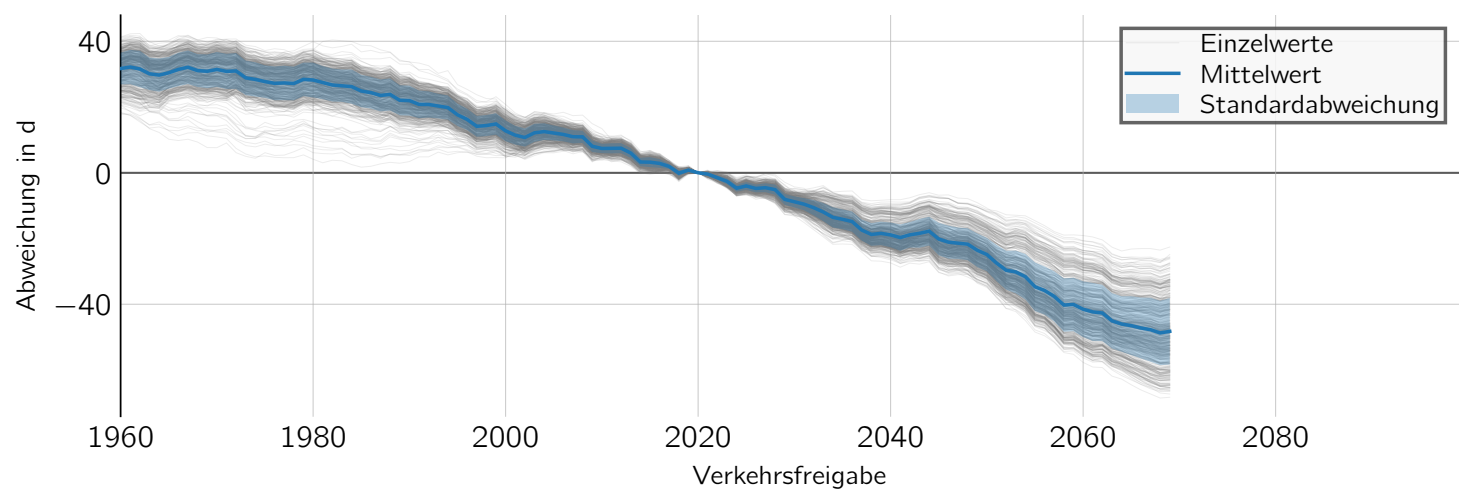

Abweichung der Frosttage innerhalb der Asphaltdeckschicht bezogen auf den Basiswert mit der Verkehrsfreigabe von 2020

\section{A.2.2 Asphalttragschicht}


A Klimarisiken

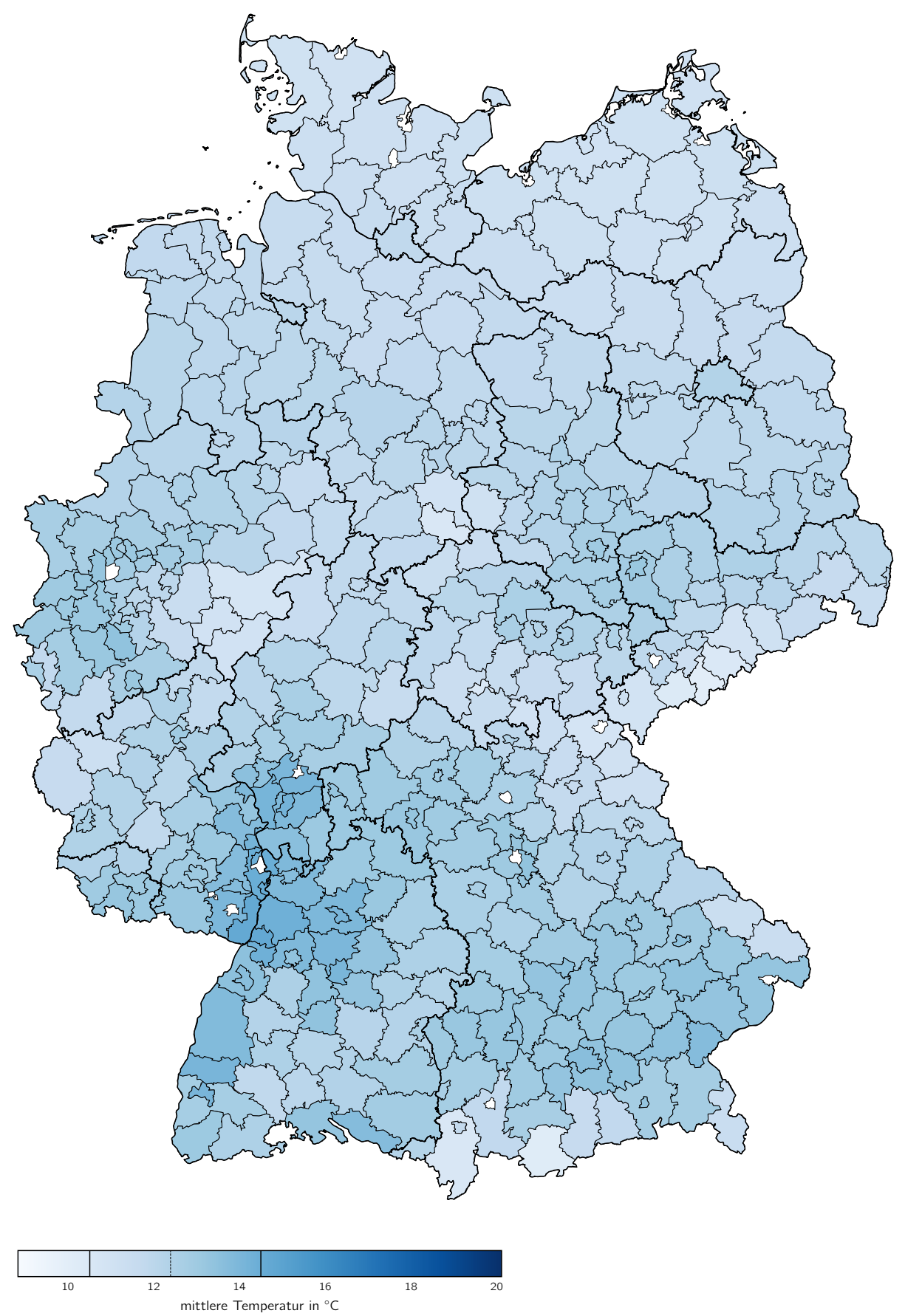

Mittlere Temperatur innerhalb der Asphalttragschicht, Verkehrsfreigabe 1960 


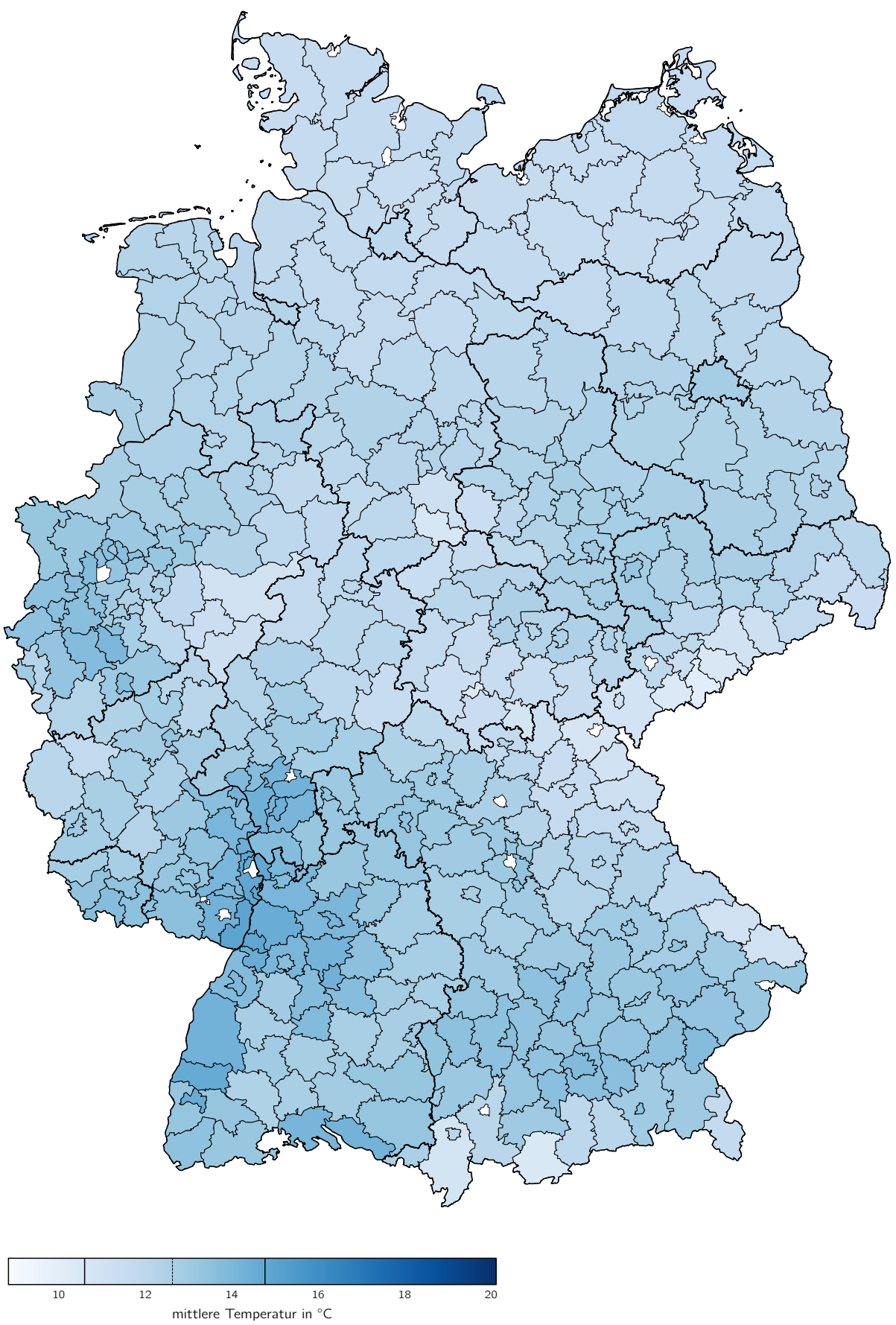

Mittlere Temperatur innerhalb der Asphalttragschicht, Verkehrsfreigabe 2020 
A Klimarisiken

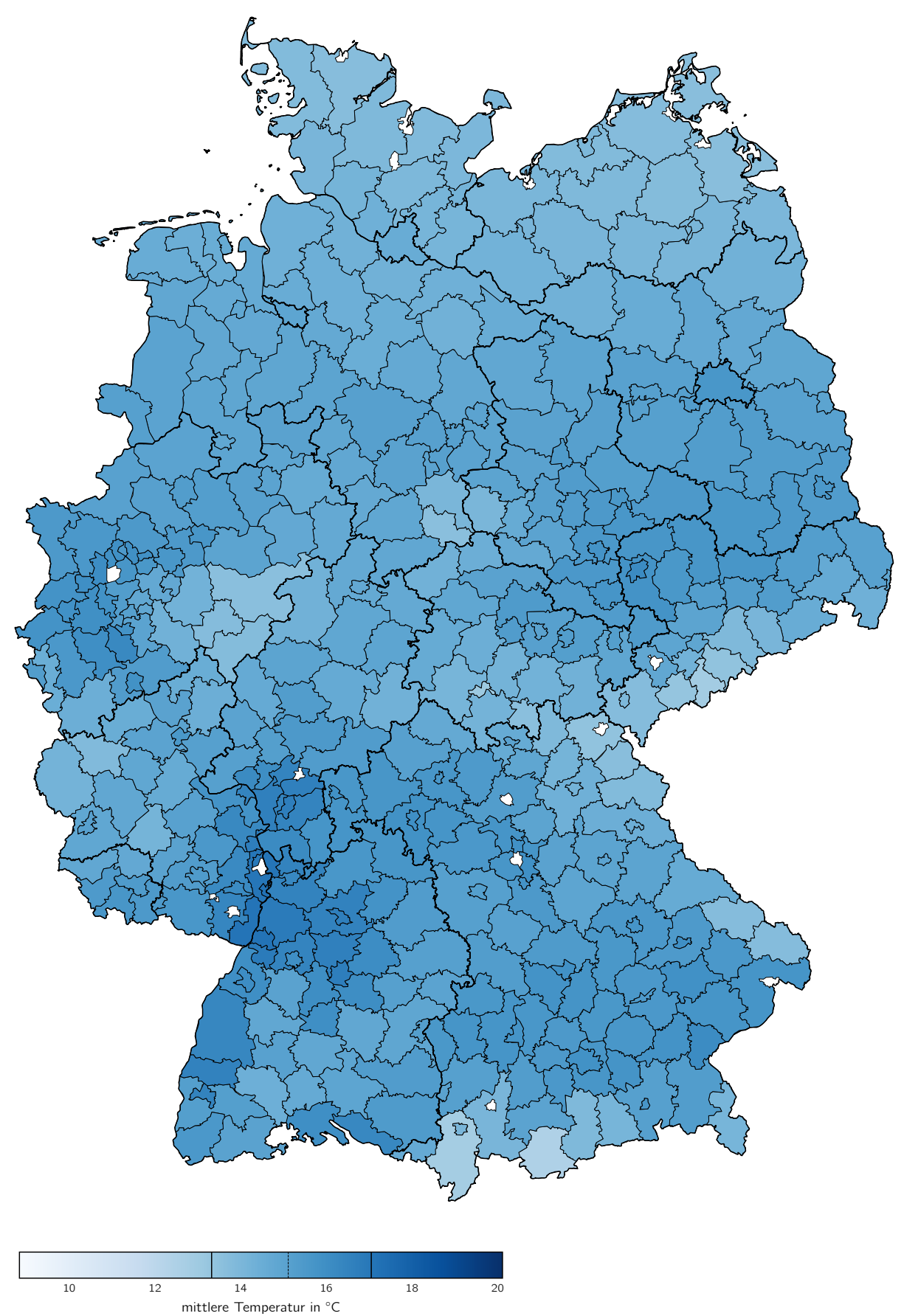

Mittlere Temperatur innerhalb der Asphalttragschicht, Verkehrsfreigabe 2040 


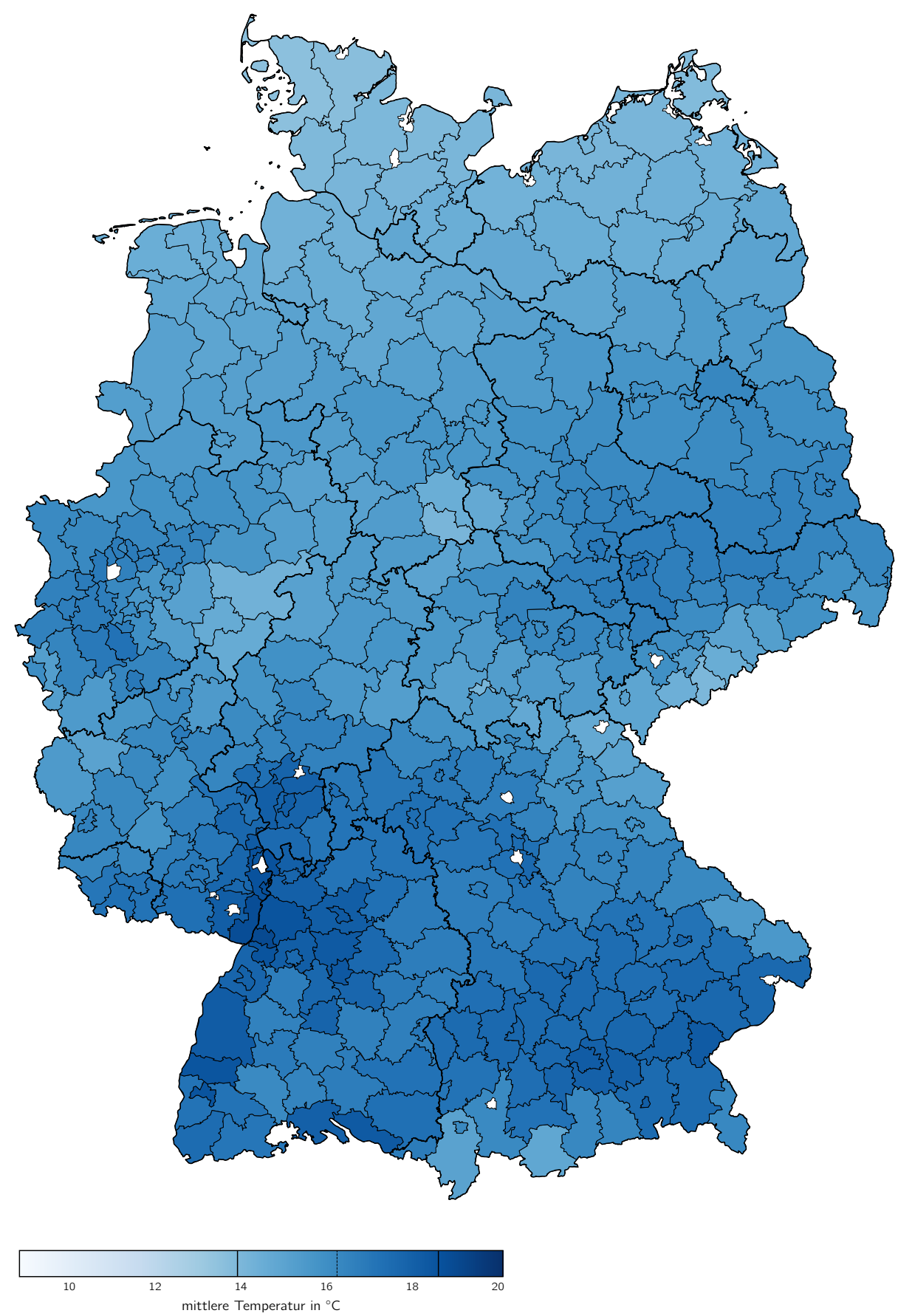

Mittlere Temperatur innerhalb der Asphalttragschicht, Verkehrsfreigabe 2070 
A Klimarisiken

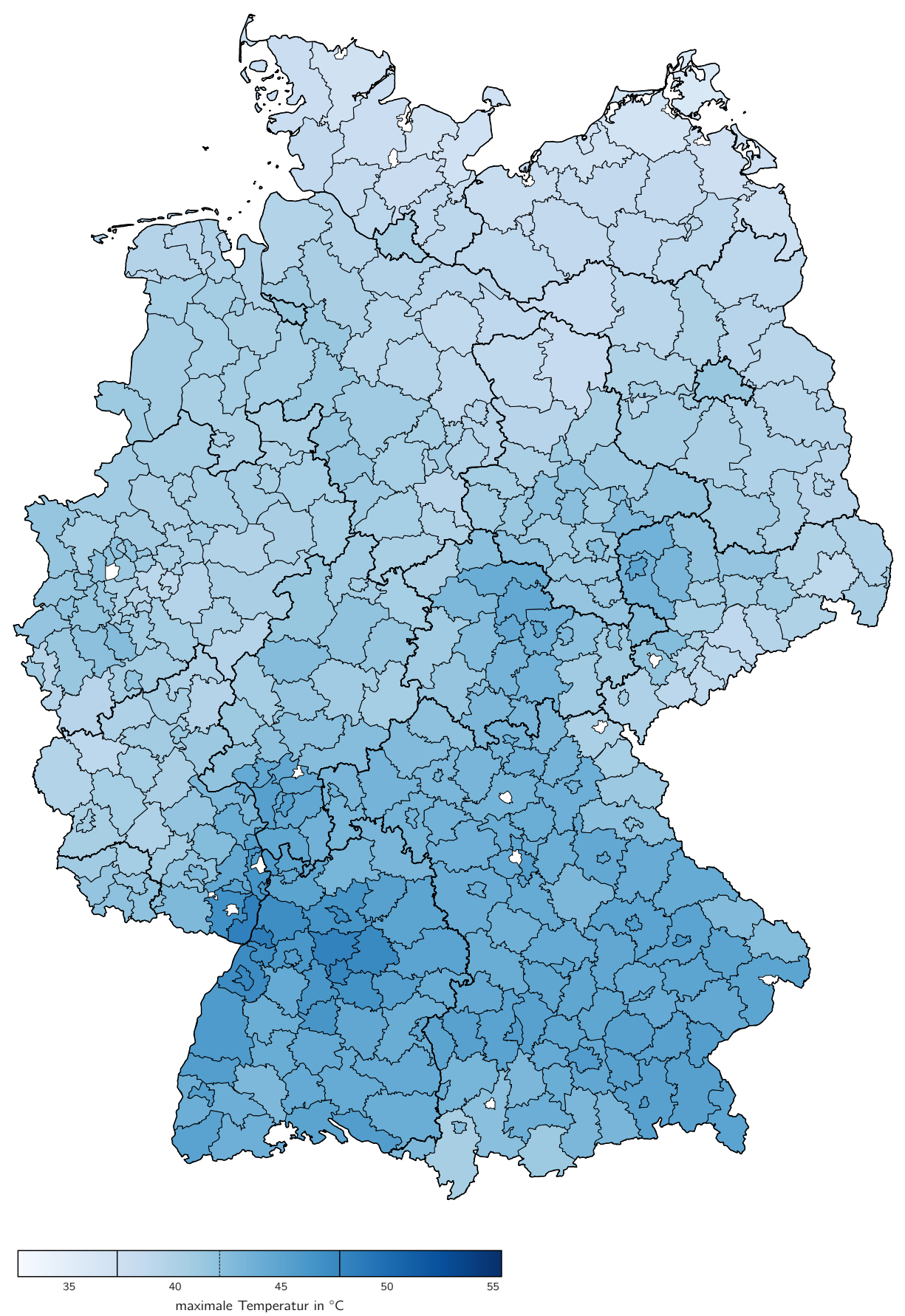

Maximale Temperaturen innerhalb der Asphalttragschicht, Verkehrsfreigabe 1960 


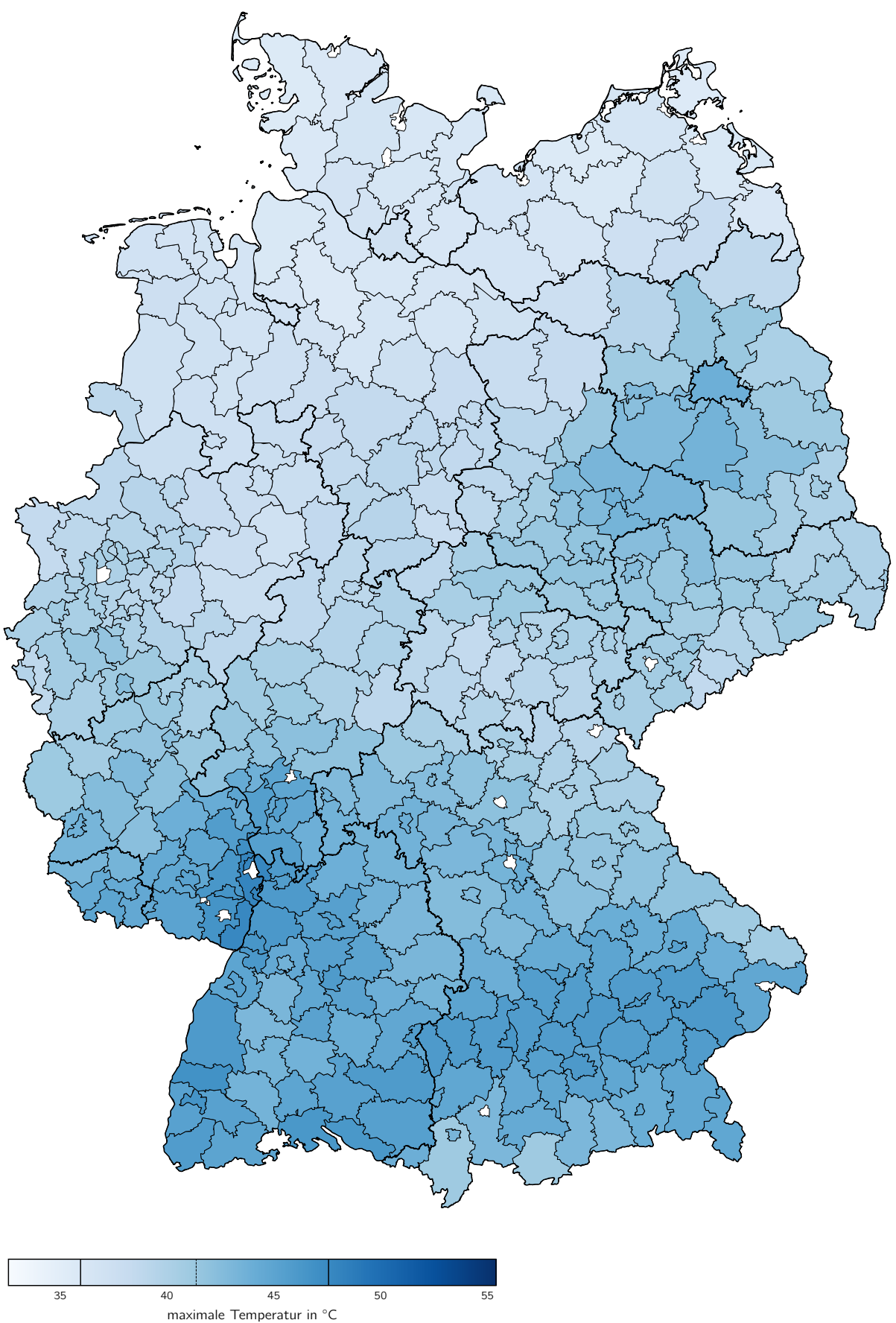

Maximale Temperaturen innerhalb der Asphalttragschicht, Verkehrsfreigabe 2020 
A Klimarisiken

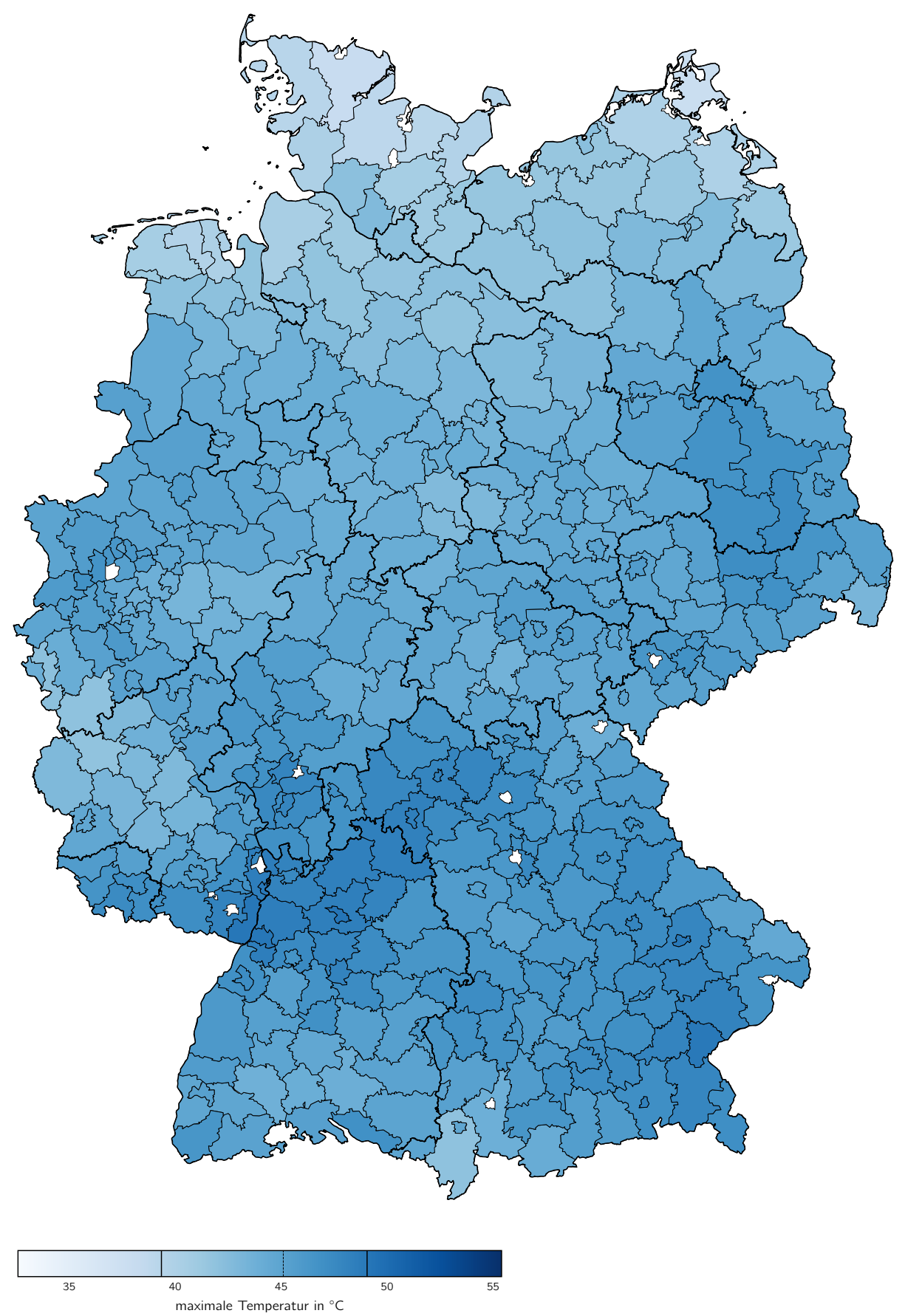

Maximale Temperaturen innerhalb der Asphalttragschicht, Verkehrsfreigabe 2040 


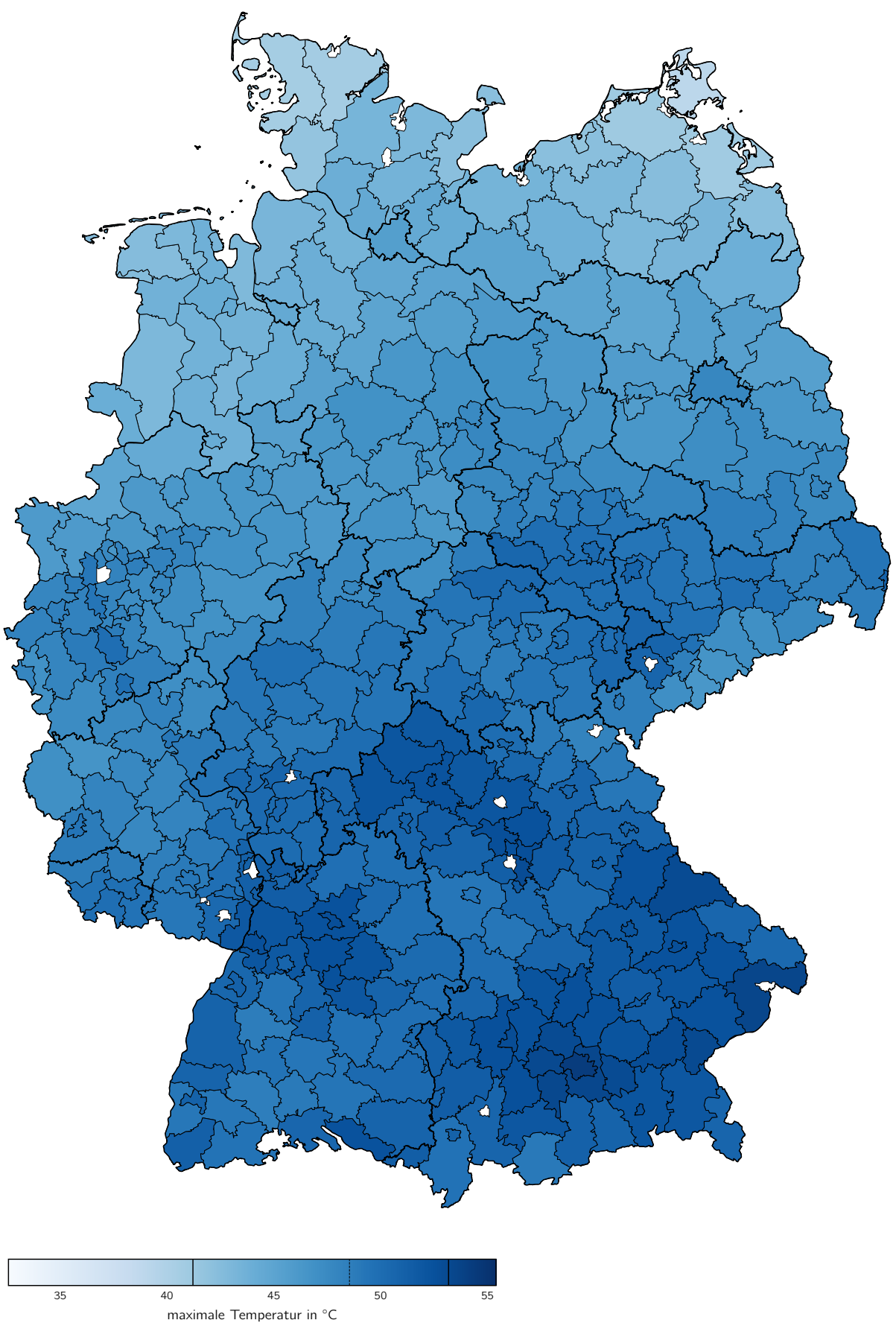

Maximale Temperaturen innerhalb der Asphalttragschicht, Verkehrsfreigabe 2070 
A Klimarisiken

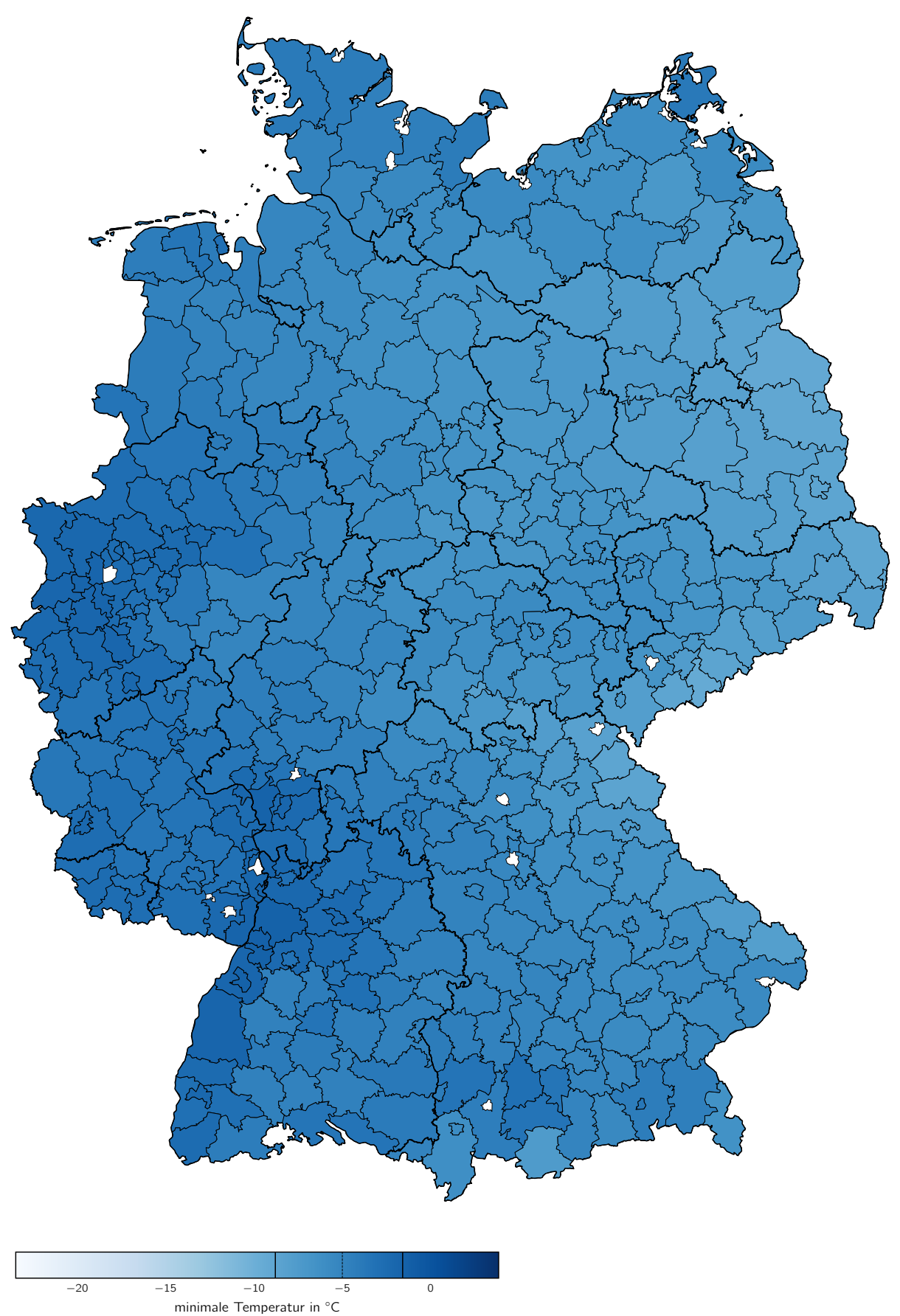

Minimale Temperaturen innerhalb der Asphalttragschicht, Verkehrsfreigabe 1960 


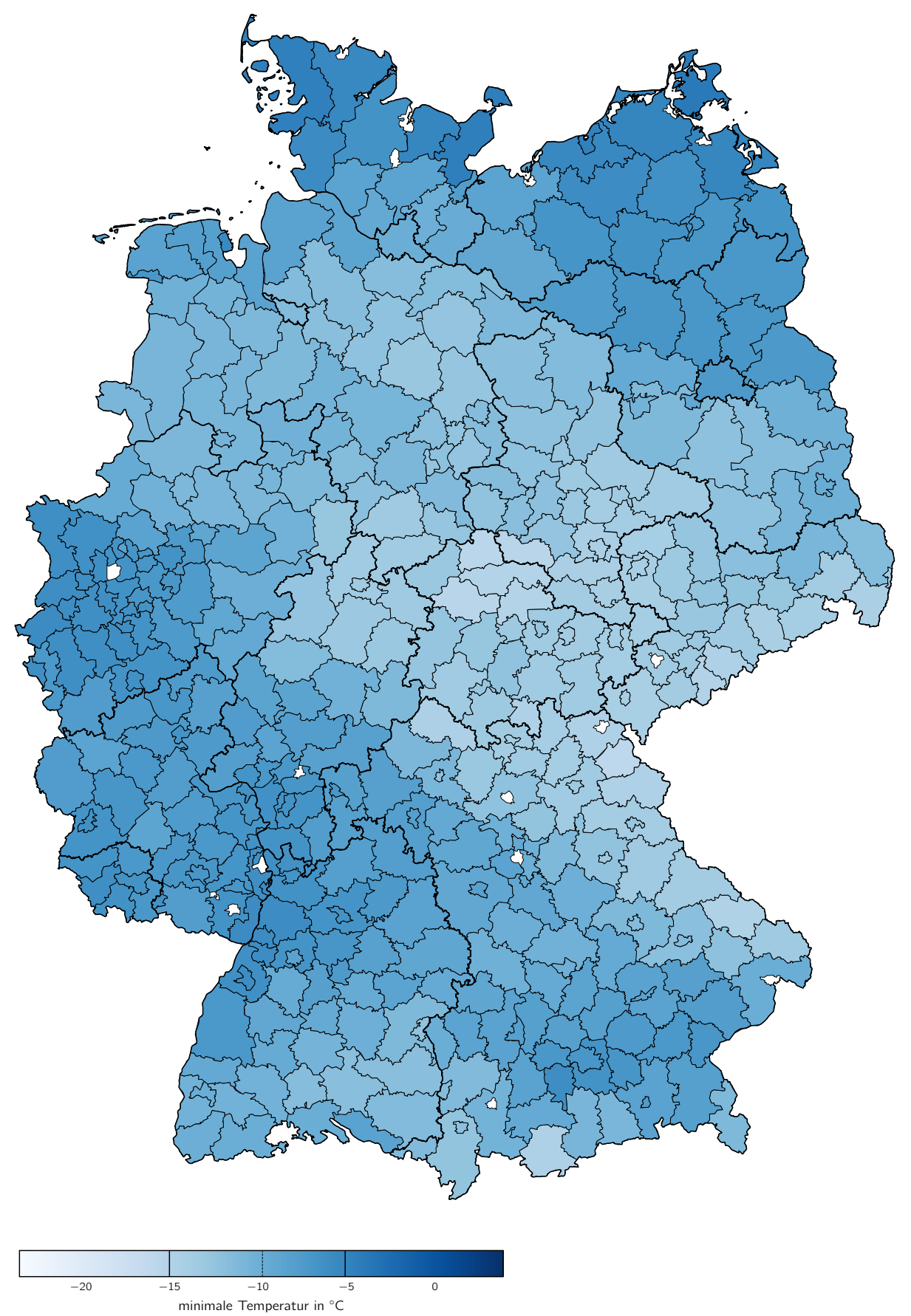

Minimale Temperaturen innerhalb der Asphalttragschicht, Verkehrsfreigabe 2020 
A Klimarisiken

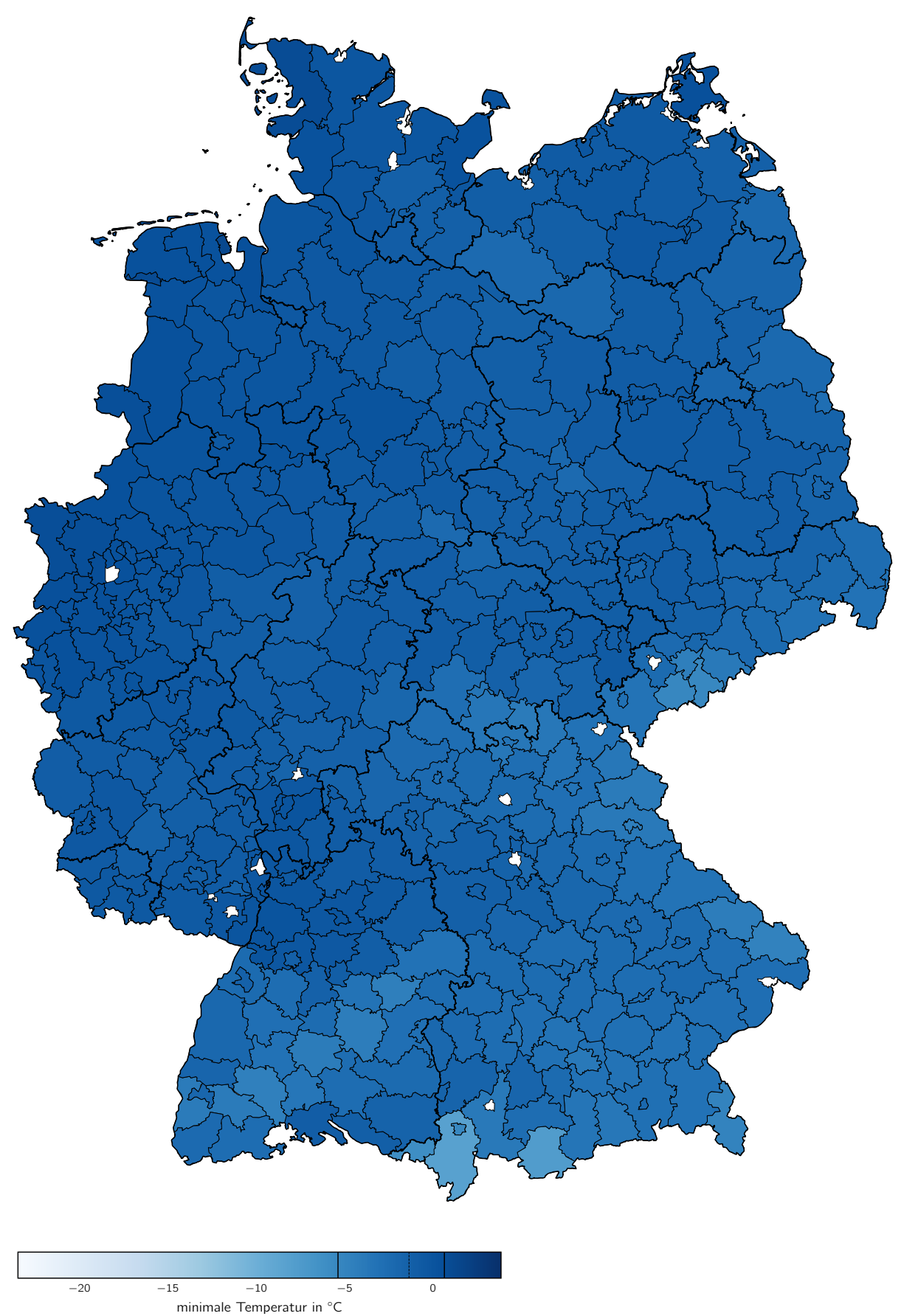

Minimale Temperaturen innerhalb der Asphalttragschicht, Verkehrsfreigabe 2040 
Anhang

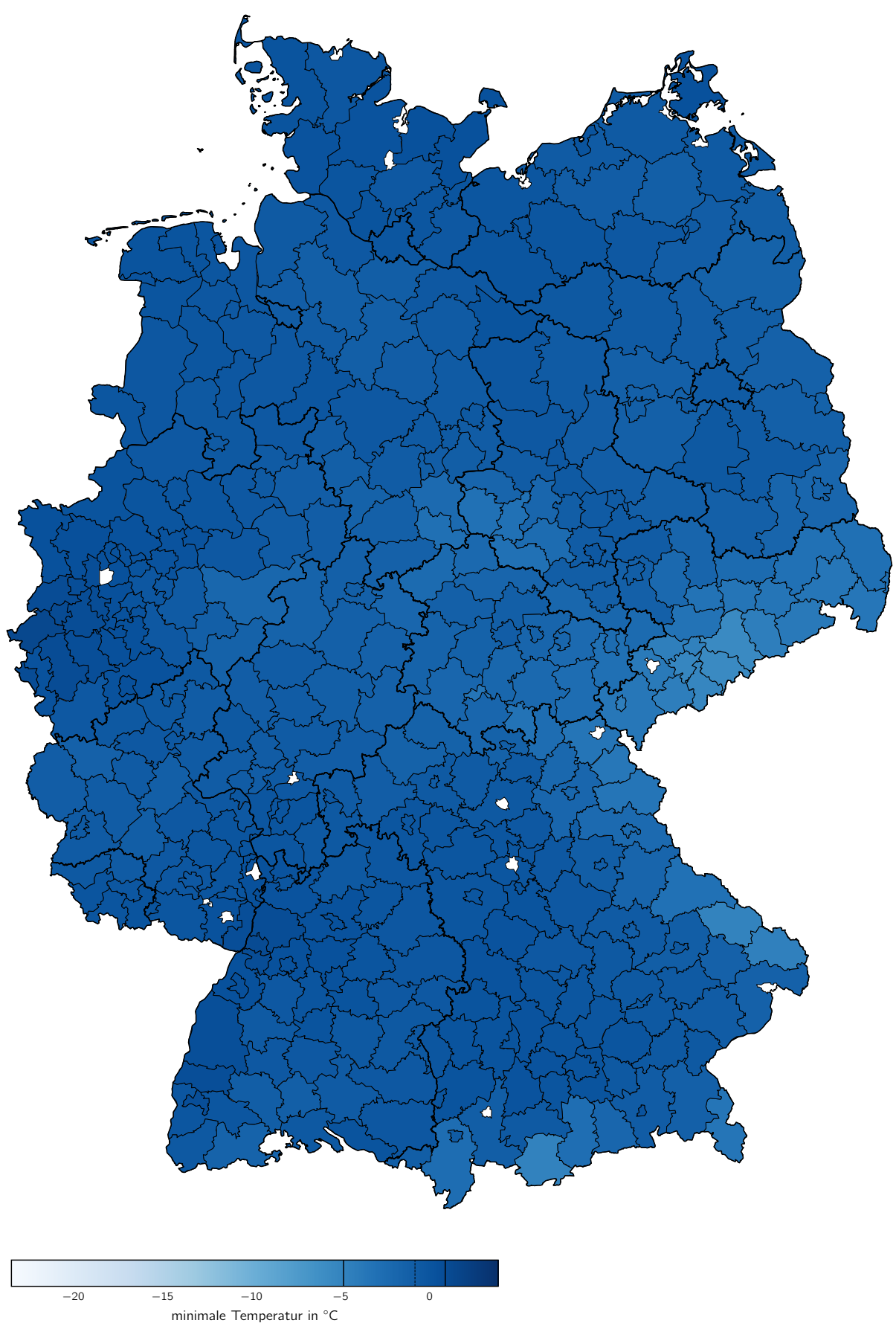

Minimale Temperaturen innerhalb der Asphalttragschicht, Verkehrsfreigabe 2070 
A Klimarisiken

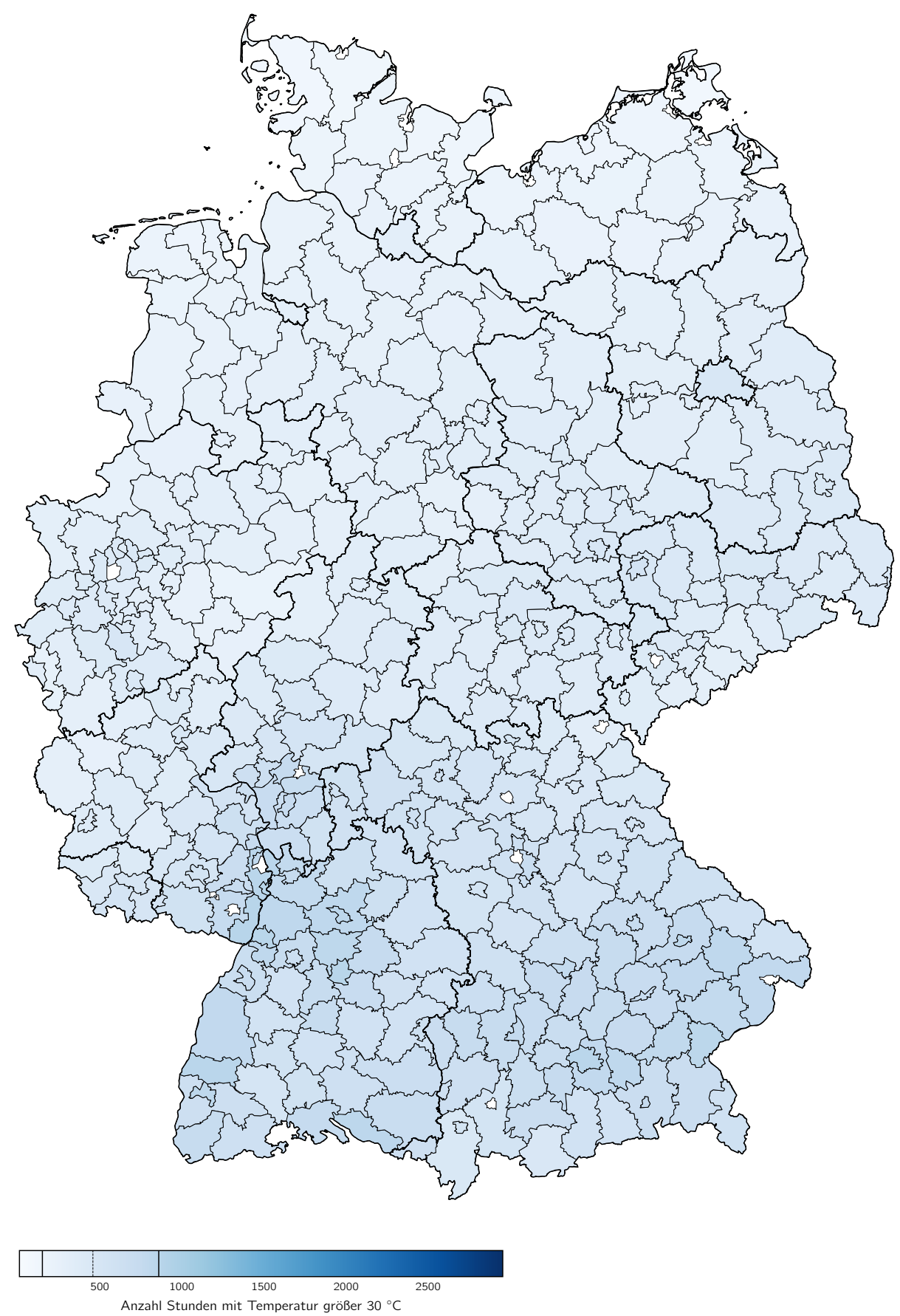

Gesamtzahl an Stunden in der die Temperatur innerhalb der Asphalttragschicht größer als $30{ }^{\circ} \mathrm{C}$ vorherrscht, Verkehrsfreigabe 1960 


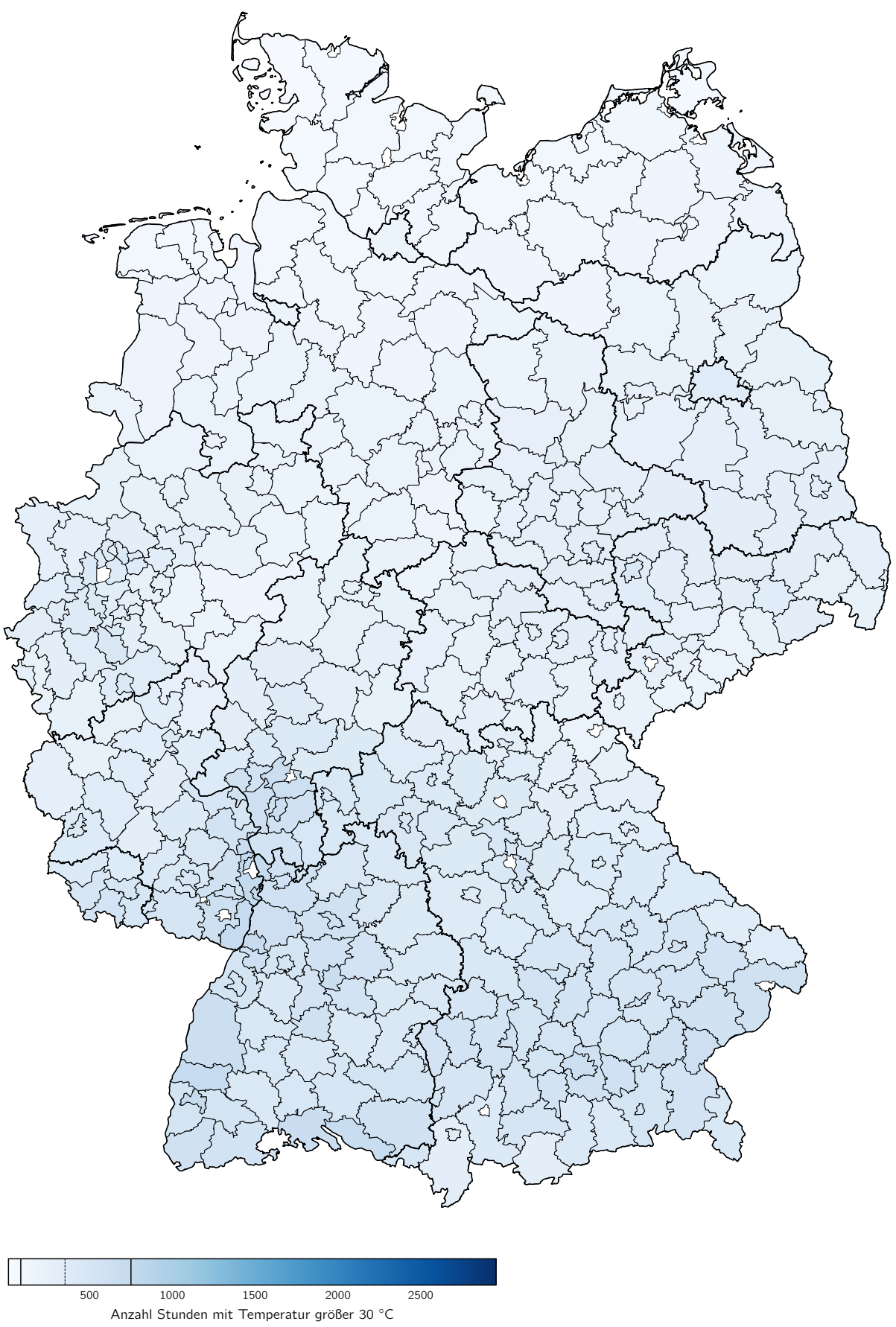

Gesamtzahl an Stunden in der die Temperatur innerhalb der Asphalttragschicht größer als $30{ }^{\circ} \mathrm{C}$ vorherrscht, Verkehrsfreigabe 2020 
A Klimarisiken

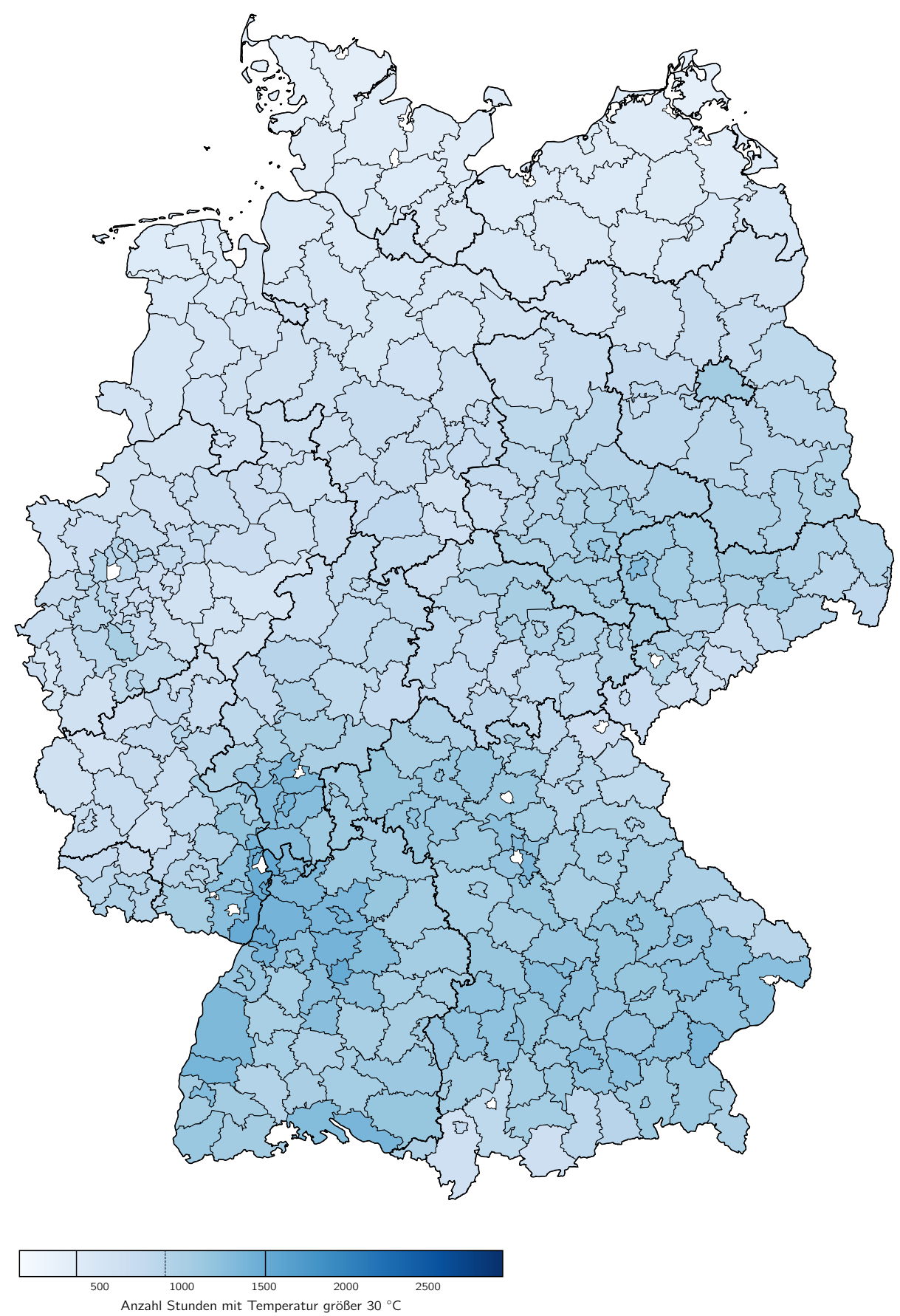

Gesamtzahl an Stunden in der die Temperatur innerhalb der Asphalttragschicht größer als $30{ }^{\circ} \mathrm{C}$ vorherrscht, Verkehrsfreigabe 2040 


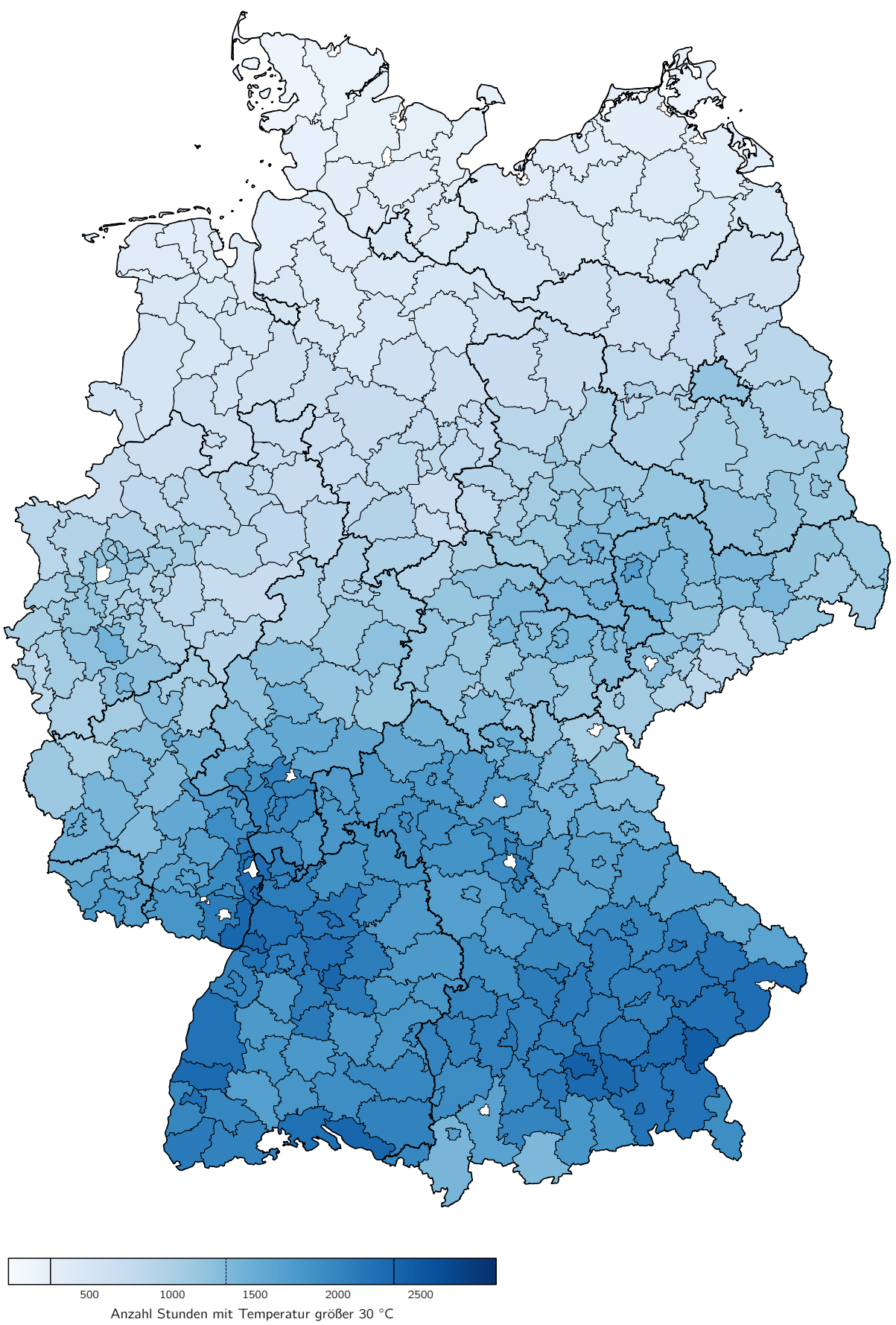

Gesamtzahl an Stunden in der die Temperatur innerhalb der Asphalttragschicht größer als $30{ }^{\circ} \mathrm{C}$ vorherrscht, Verkehrsfreigabe 2070 
A Klimarisiken

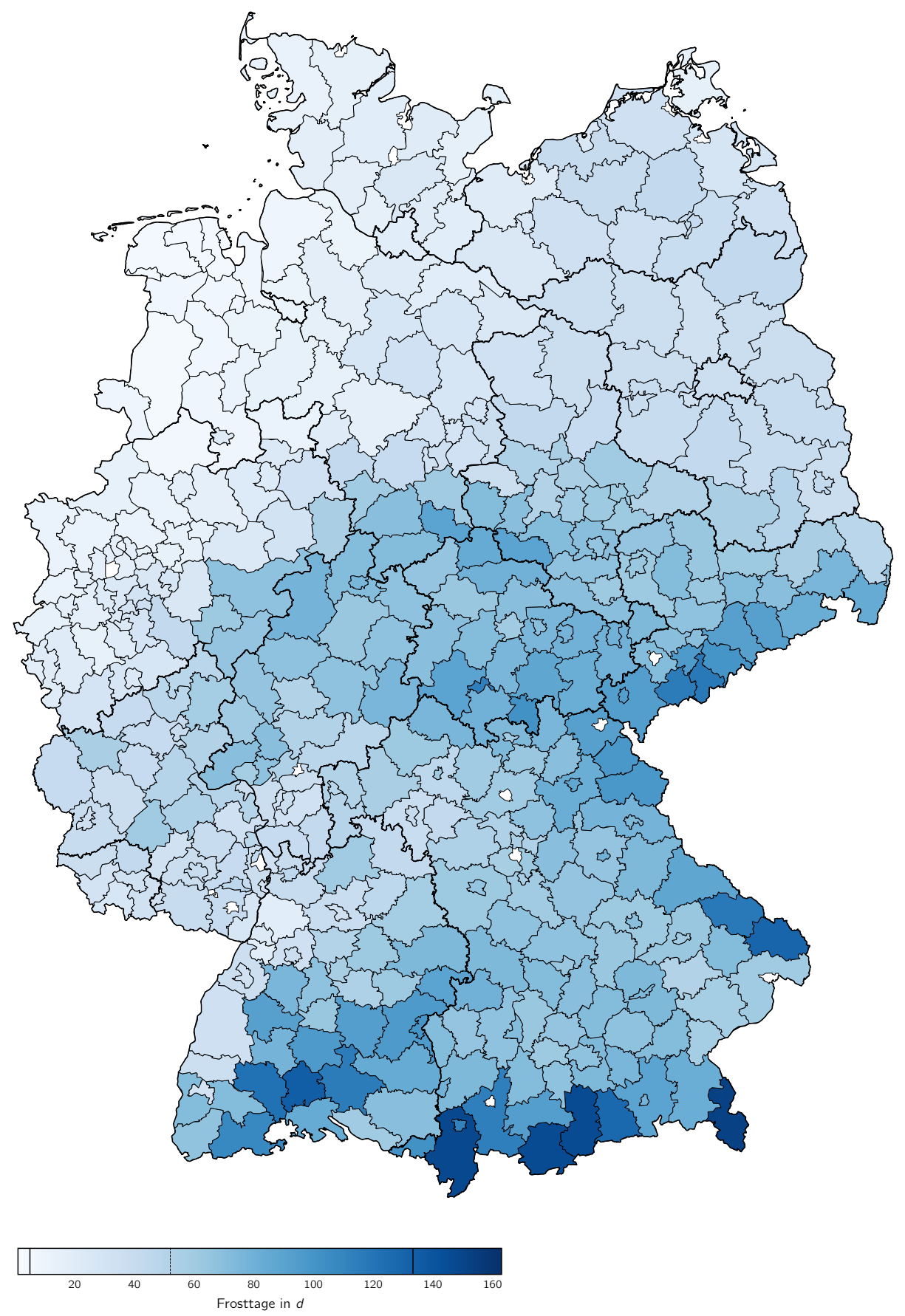

Frosttage, Asphalttragschicht, Verkehrsfreigabe 1960 


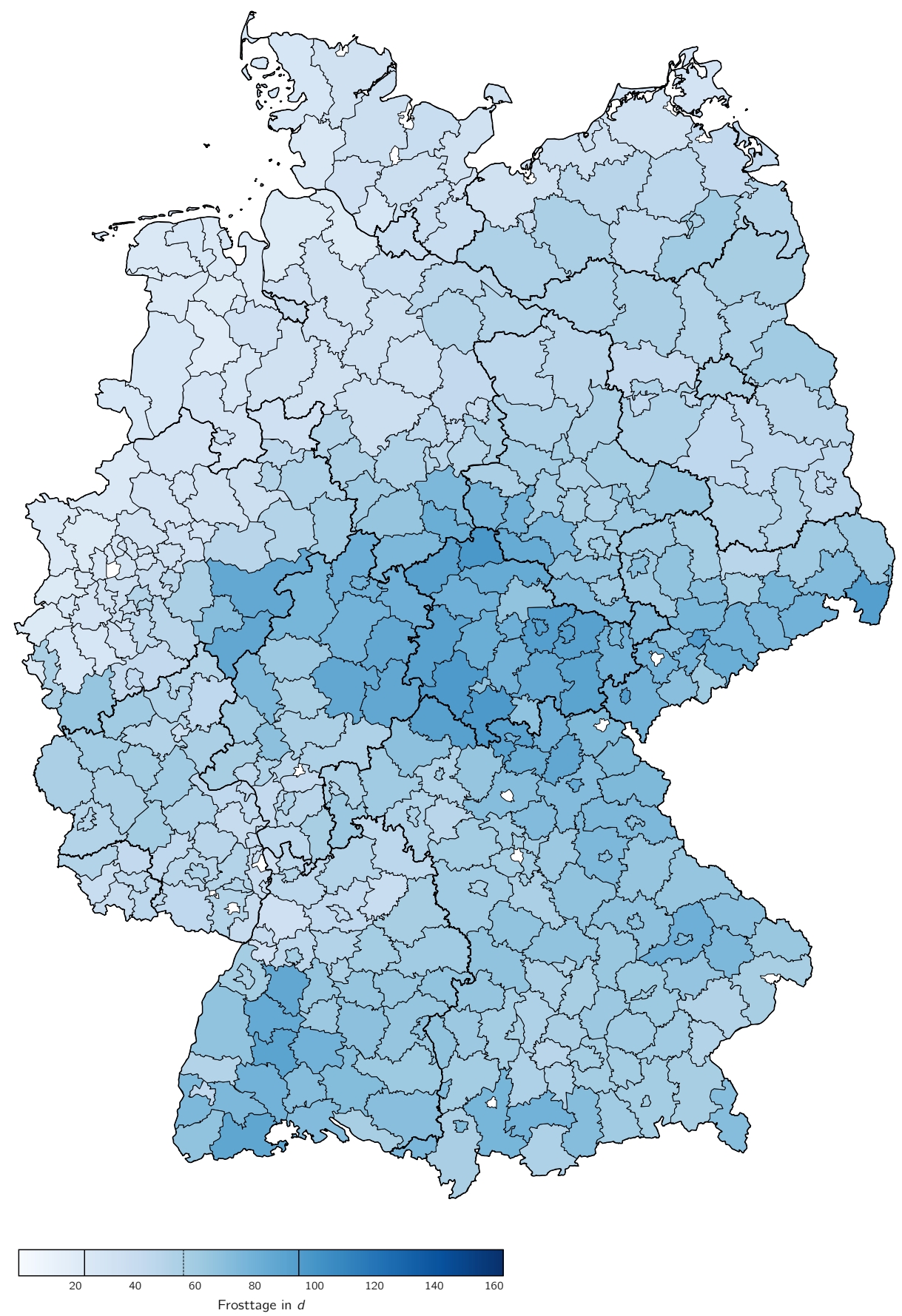

Frosttage, Asphalttragschicht, Verkehrsfreigabe 2020 


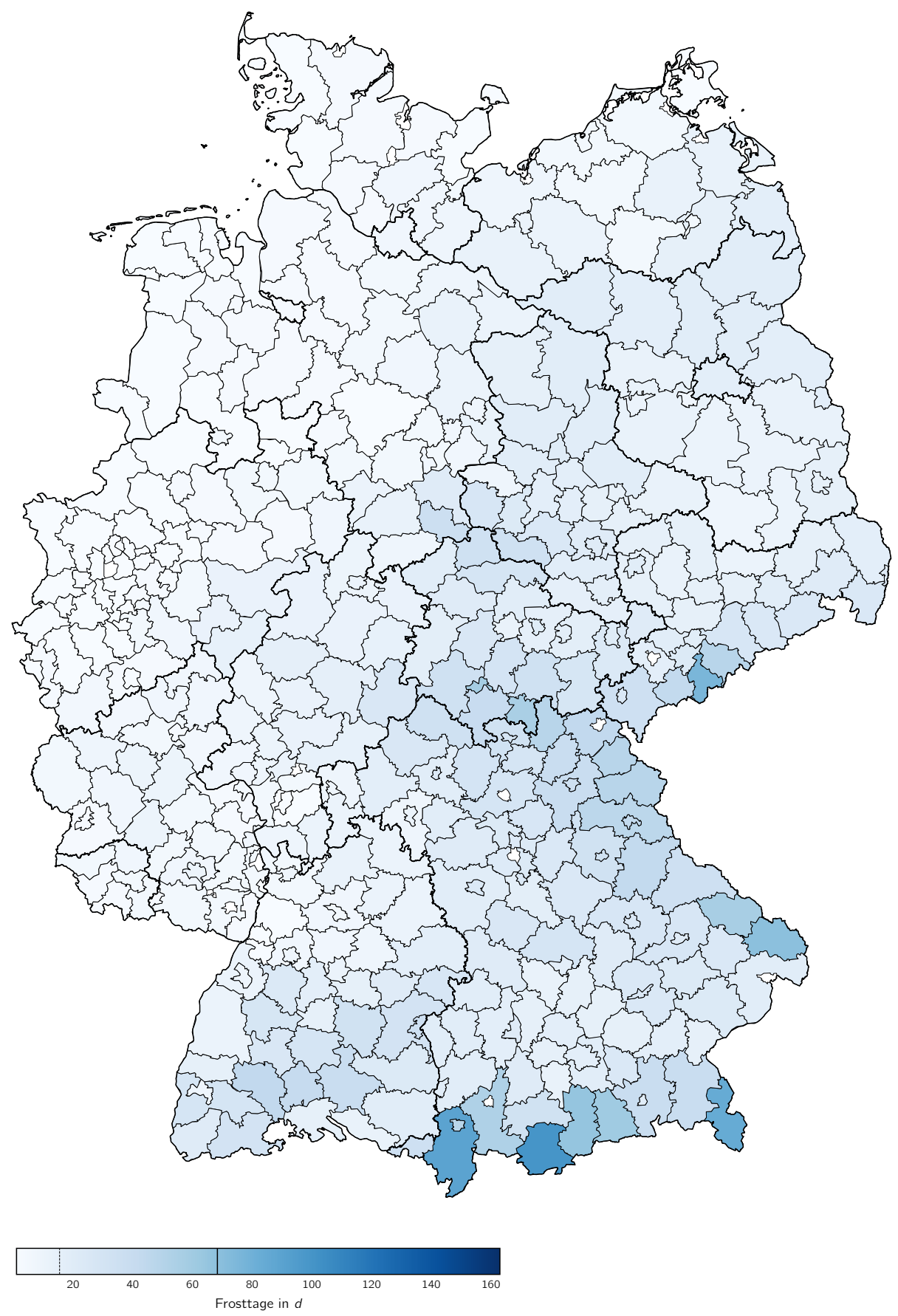

Frosttage, Asphalttragschicht, Verkehrsfreigabe 2040 


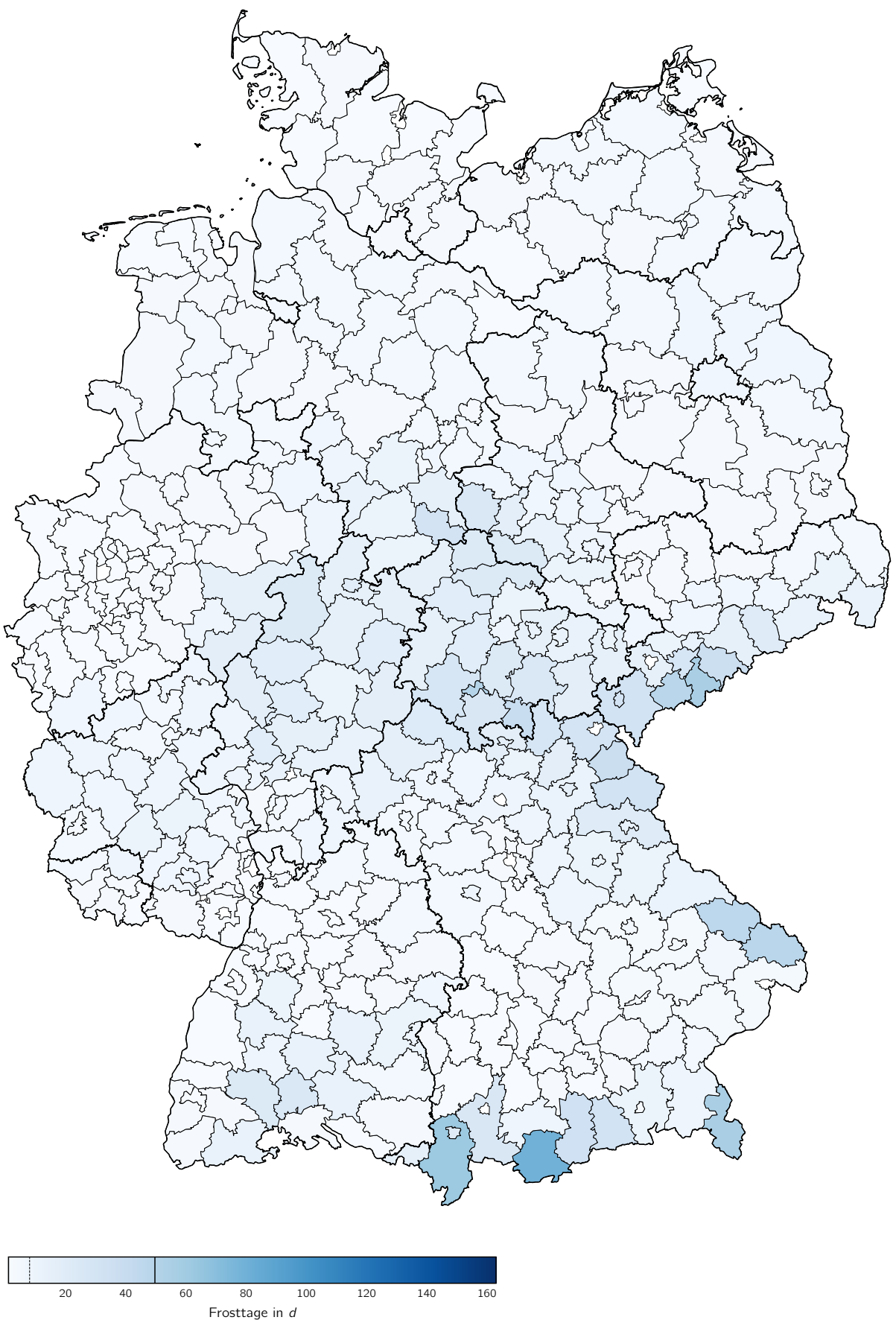

Frosttage, Asphalttragschicht, Verkehrsfreigabe 2070 


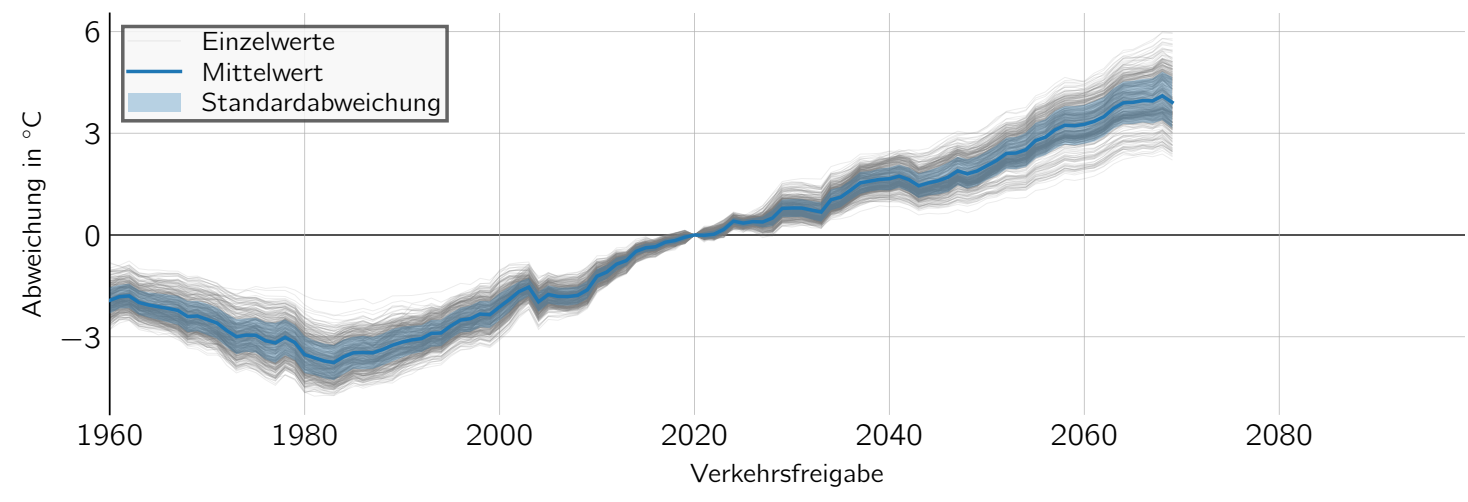

Abweichung der minimalen Temperatur innerhalb der Asphalttragschicht bezogen auf den Basiswert mit der Verkehrsfreigabe von 2020

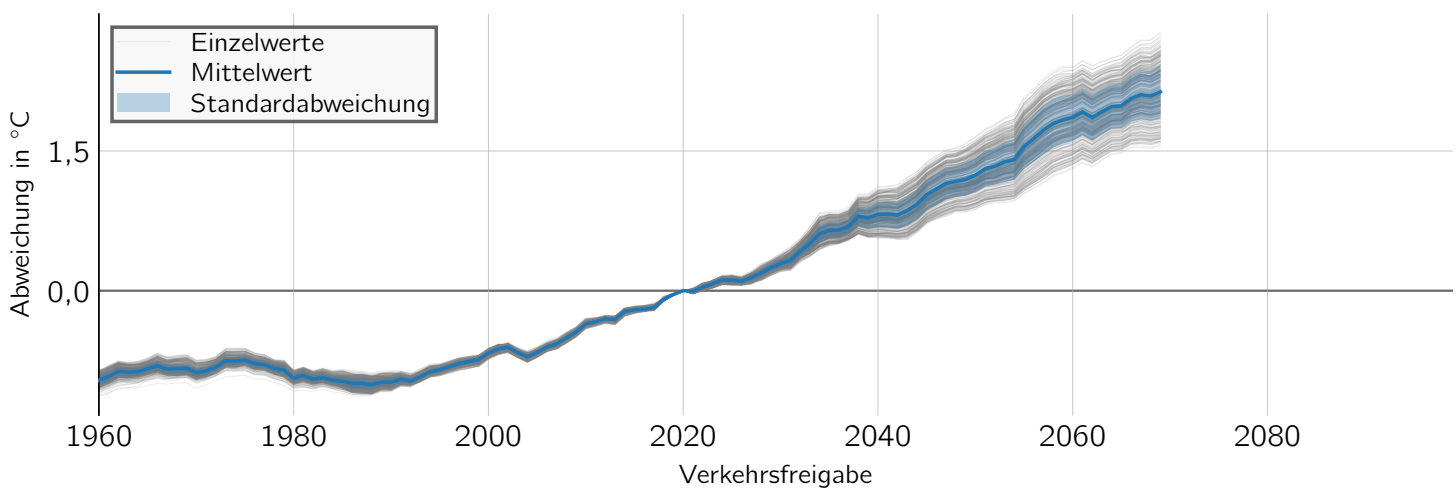

Abweichung der mittleren Temperatur innerhalb der Asphalttragschicht bezogen auf den Basiswert mit der Verkehrsfreigabe von 2020

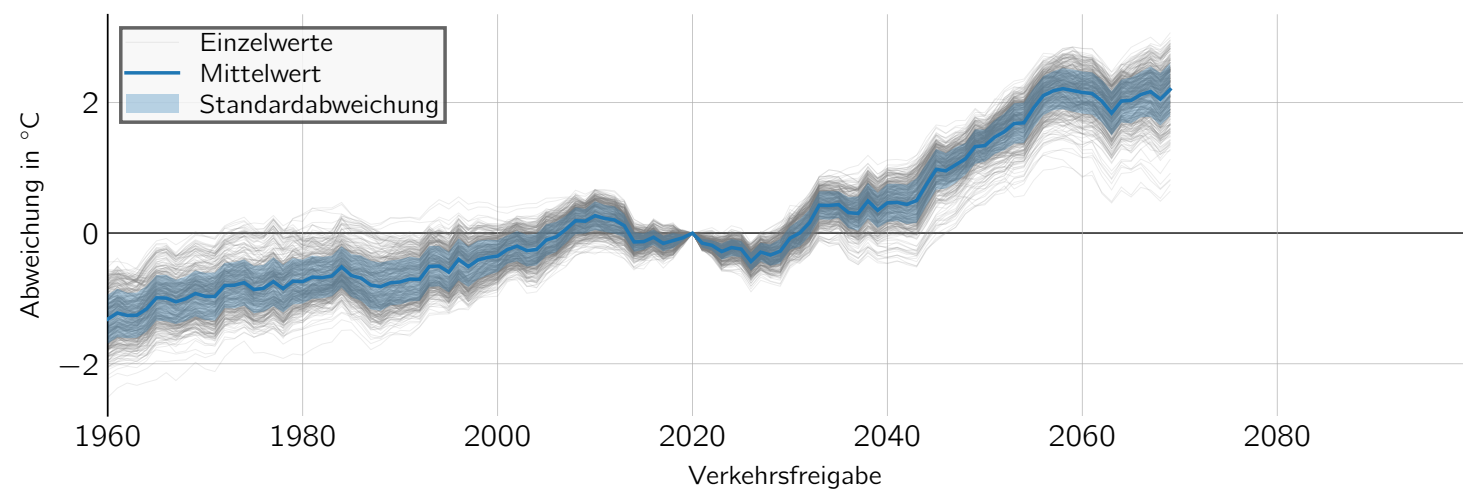

Abweichung der maximalen Temperatur innerhalb der Asphalttragschicht bezogen auf den Basiswert mit der Verkehrsfreigabe von 2020 


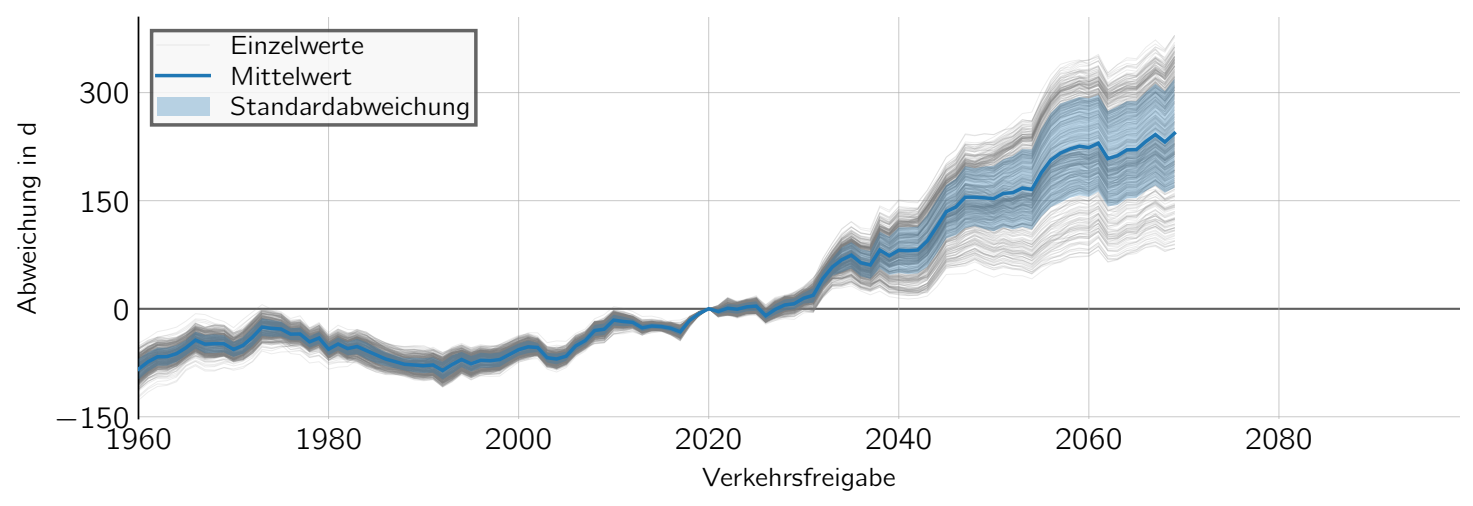

Abweichung der maximalen Temperatur innerhalb der Asphaltdeckschicht bezogen auf den Basiswert mit der Verkehrsfreigabe von 2020

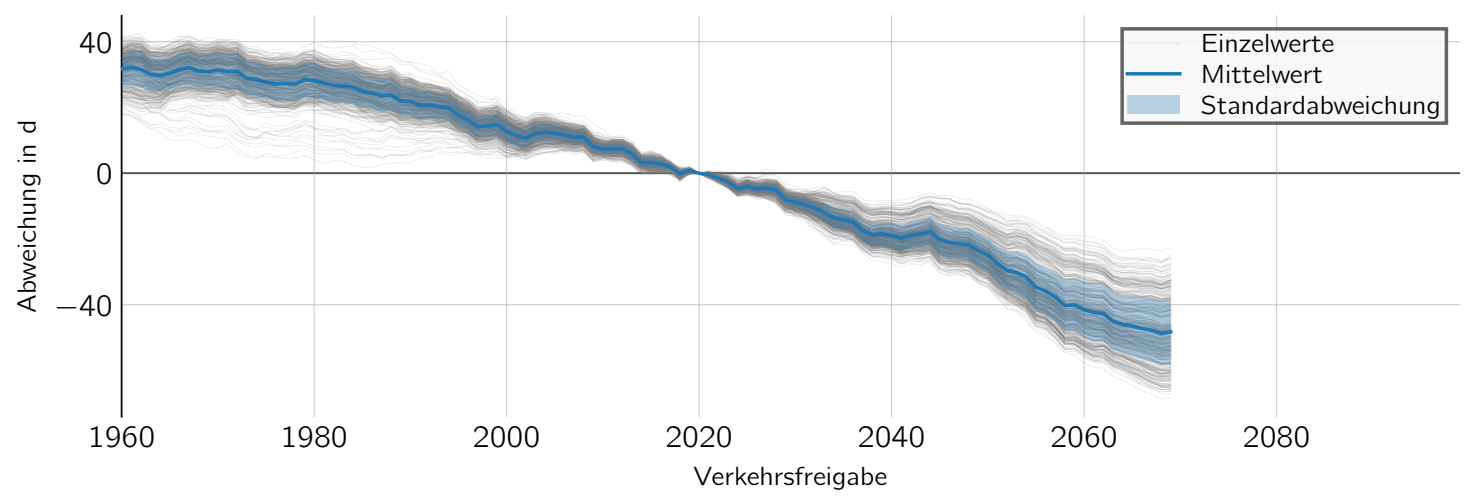

Abweichung der Frosttage innerhalb der Asphaltdeckschicht bezogen auf den Basiswert mit der Verkehrsfreigabe von 2020 


\section{B Clusteranalyse}

\section{B.1 Mittelwertfunktionen}

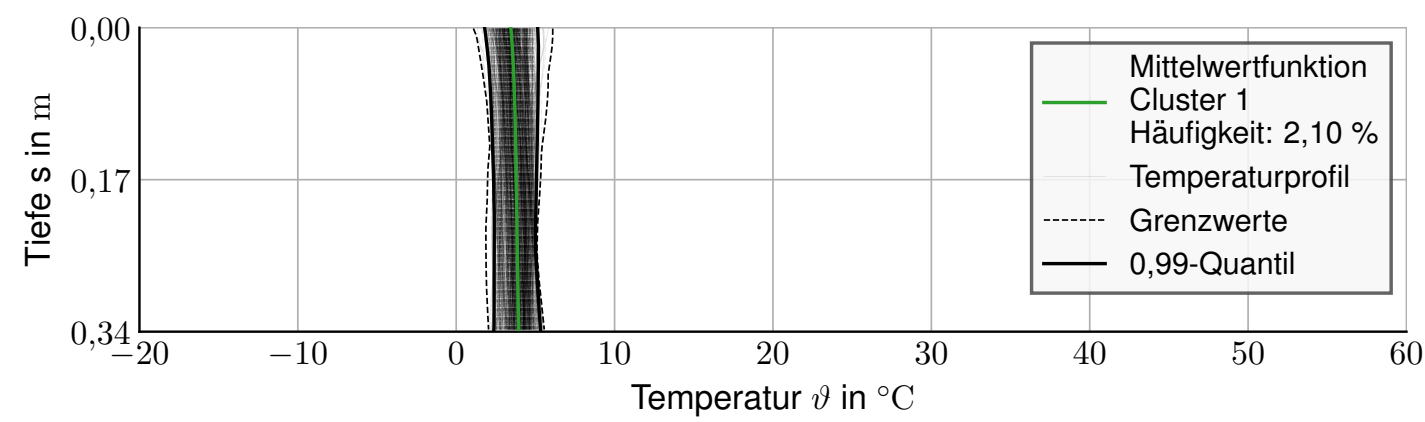

Abweichung zwischen den simulierten Temperaturbedingungen und dem Funktionsverlauf der Mittelwertfunktion, Cluster 0

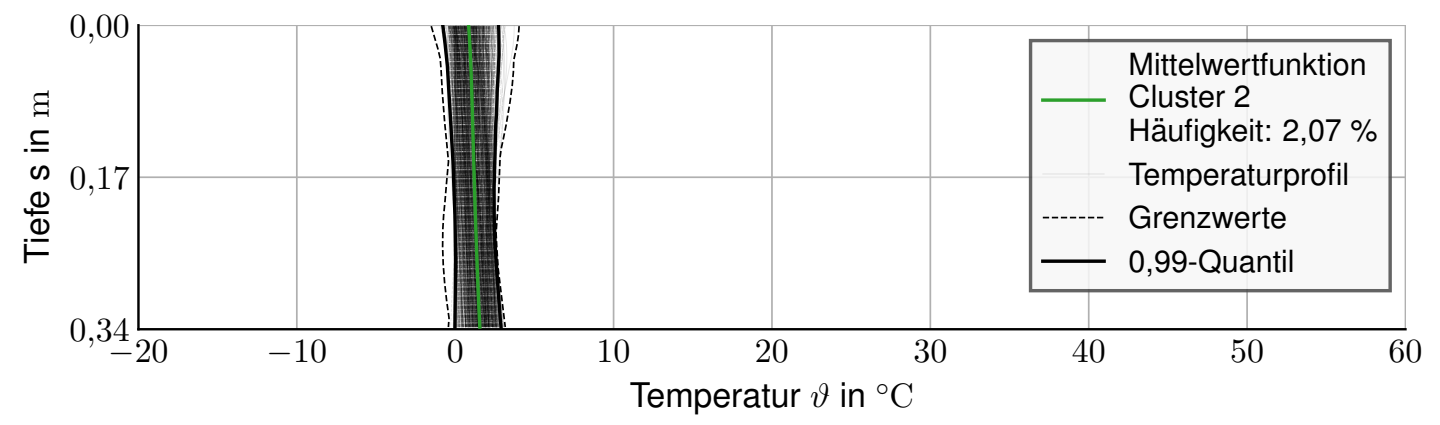

Abweichung zwischen den simulierten Temperaturbedingungen und dem Funktionsverlauf der Mittelwertfunktion, Cluster 1 


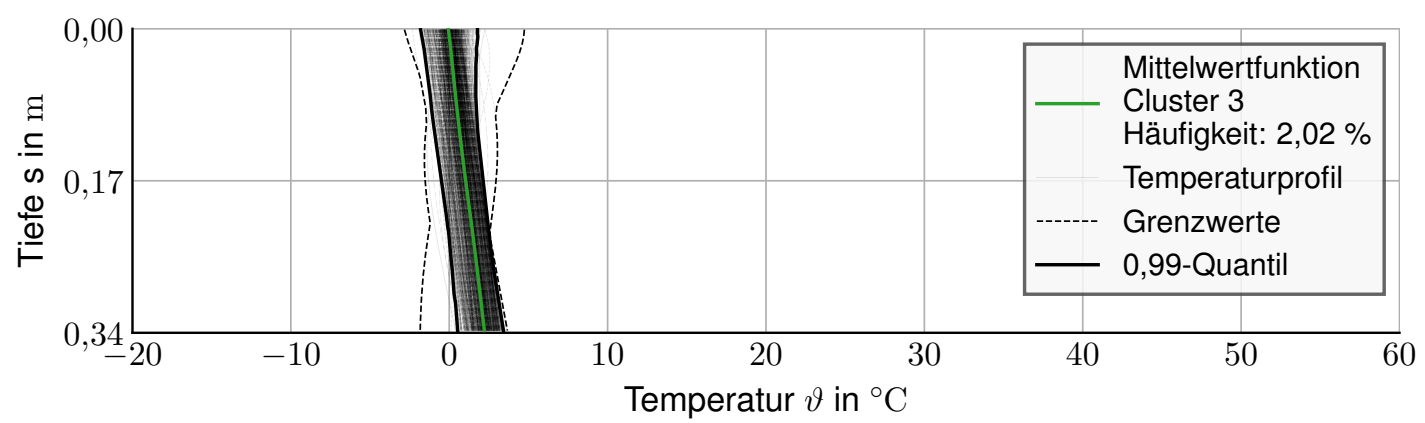

Abweichung zwischen den simulierten Temperaturbedingungen und dem Funktionsverlauf der Mittelwertfunktion, Cluster 2

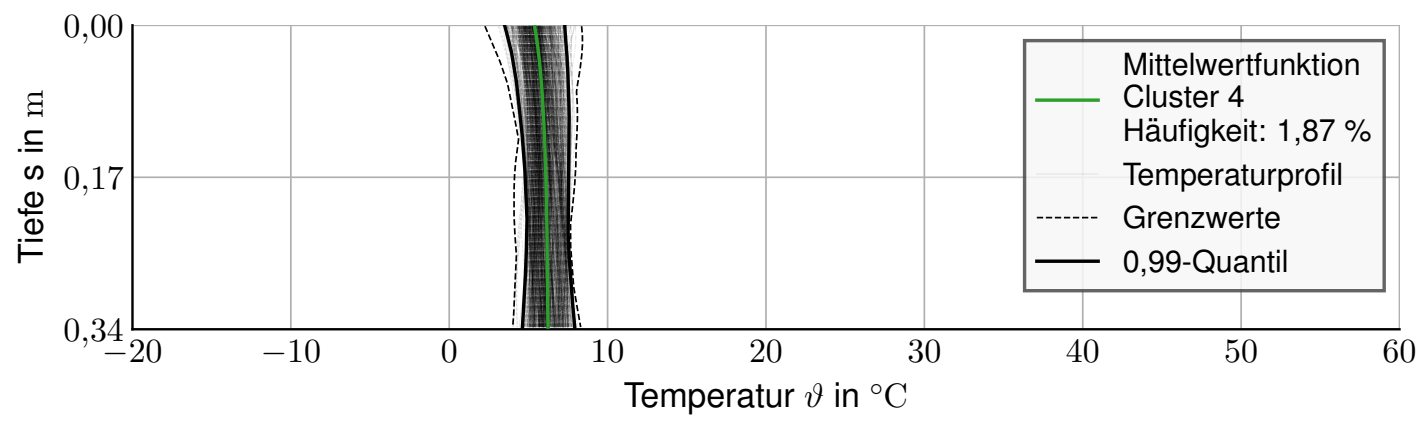

Abweichung zwischen den simulierten Temperaturbedingungen und dem Funktionsverlauf der Mittelwertfunktion, Cluster 3

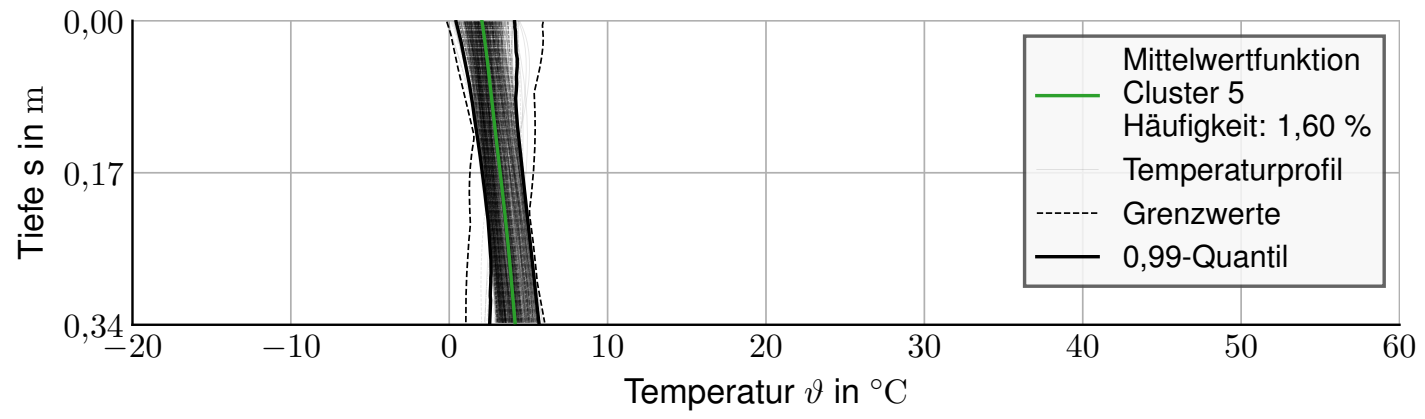

Abweichung zwischen den simulierten Temperaturbedingungen und dem Funktionsverlauf der Mittelwertfunktion, Cluster 4 


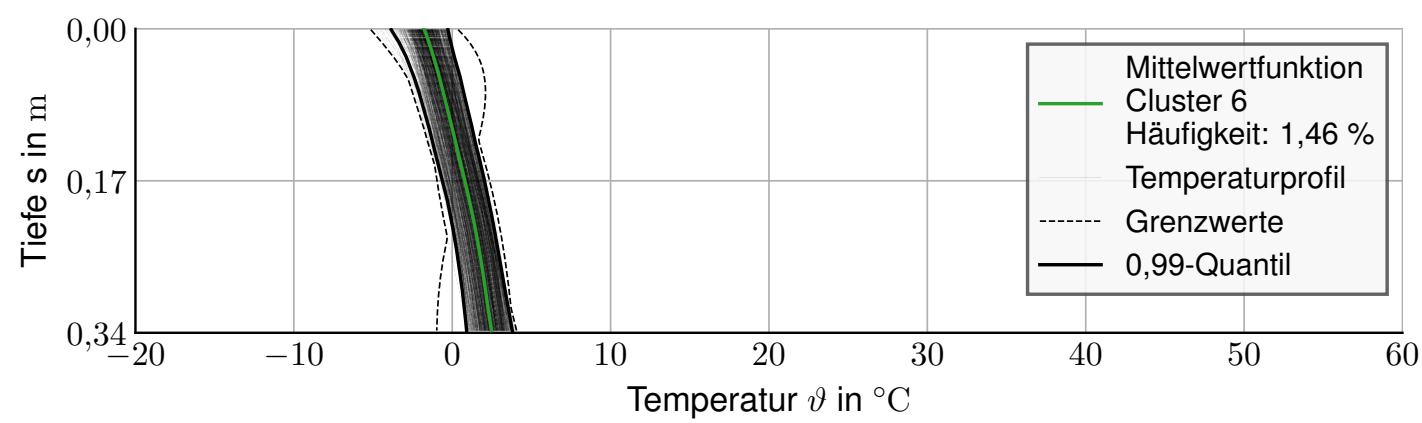

Abweichung zwischen den simulierten Temperaturbedingungen und dem Funktionsverlauf der Mittelwertfunktion, Cluster 5

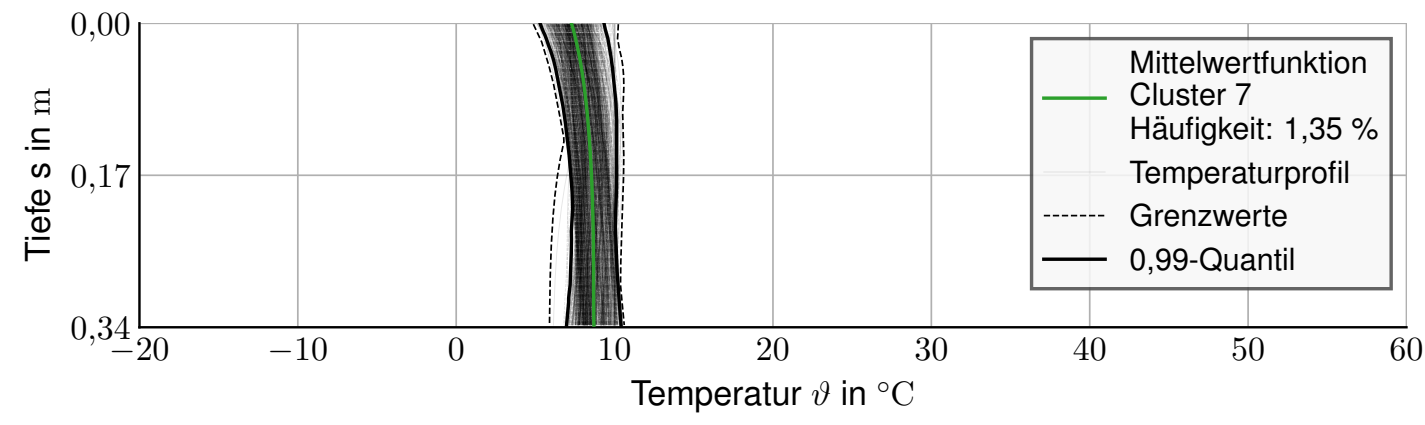

Abweichung zwischen den simulierten Temperaturbedingungen und dem Funktionsverlauf der Mittelwertfunktion, Cluster 6

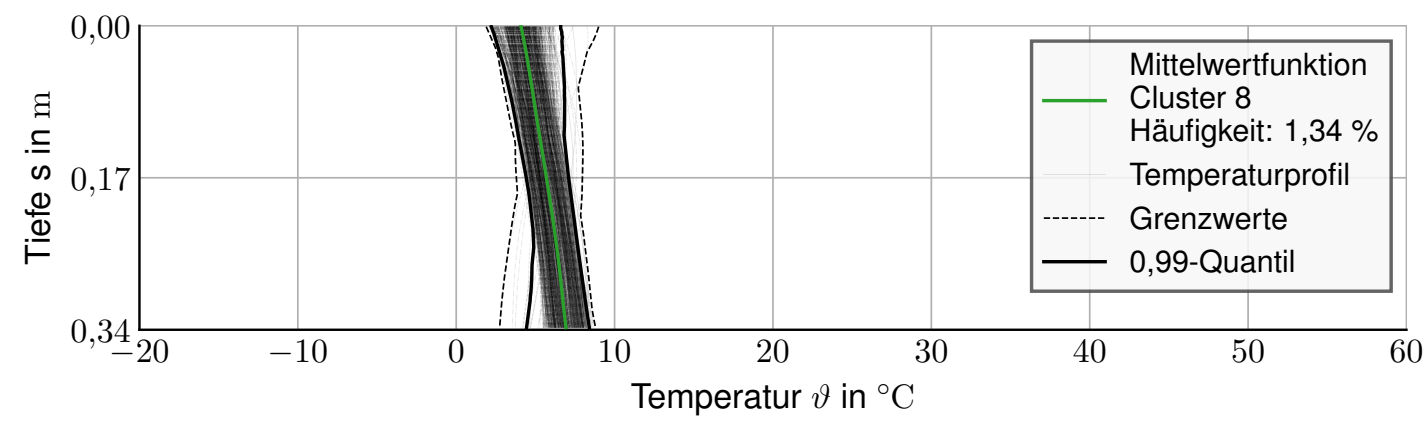

Abweichung zwischen den simulierten Temperaturbedingungen und dem Funktionsverlauf der Mittelwertfunktion, Cluster 7 


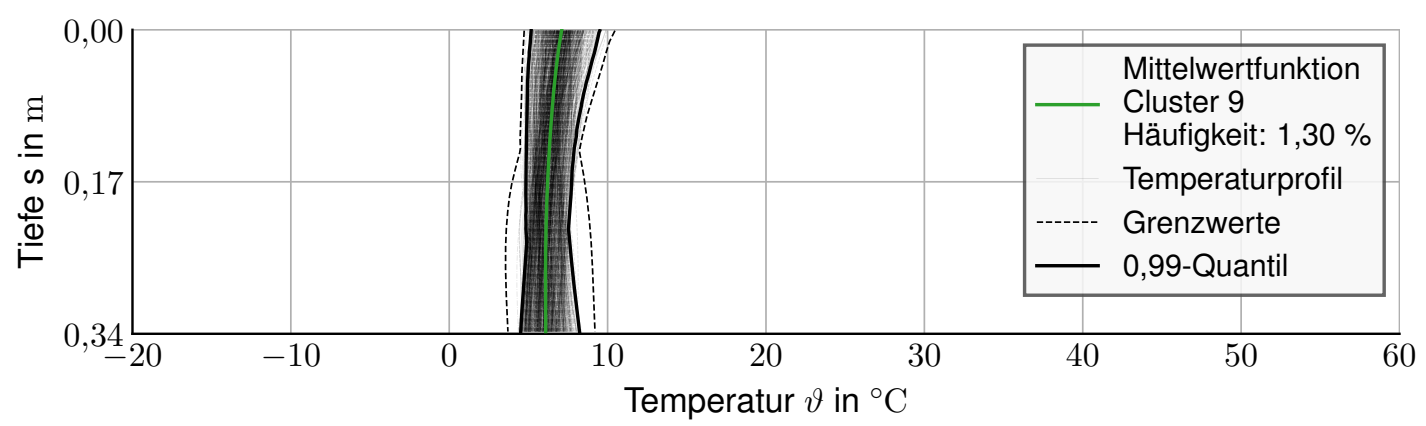

Abweichung zwischen den simulierten Temperaturbedingungen und dem Funktionsverlauf der Mittelwertfunktion, Cluster 8

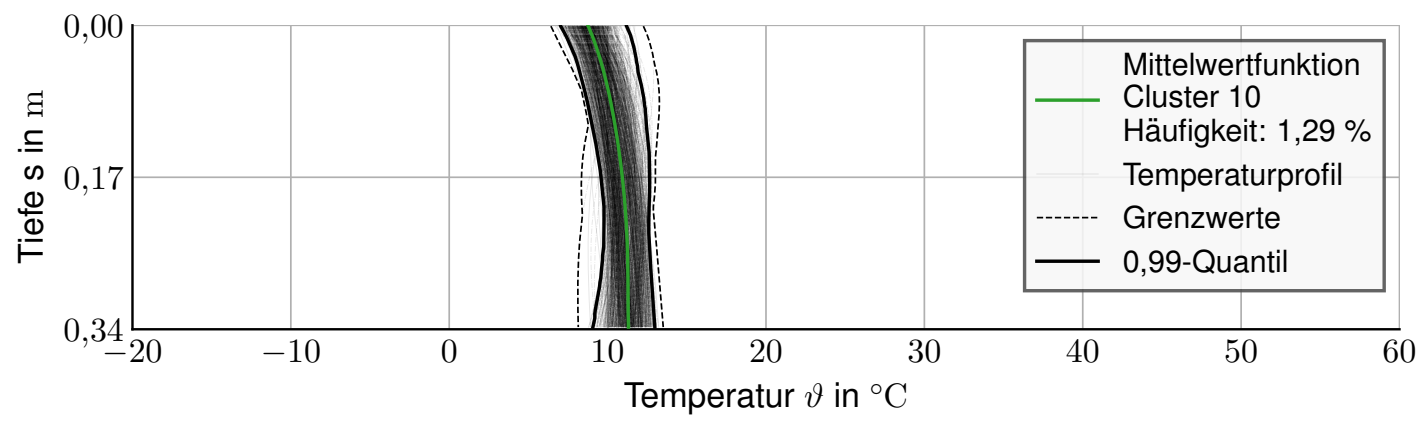

Abweichung zwischen den simulierten Temperaturbedingungen und dem Funktionsverlauf der Mittelwertfunktion, Cluster 9

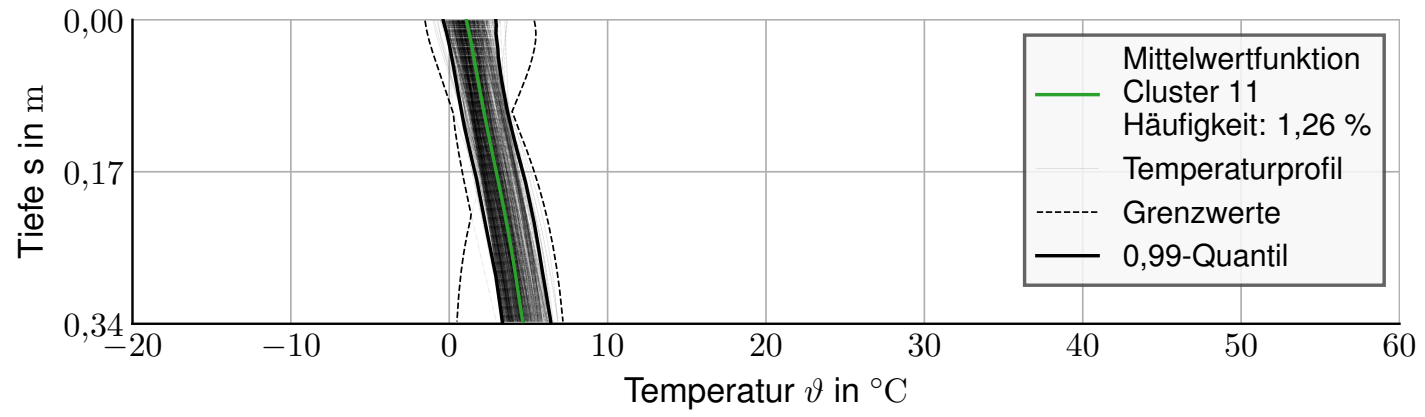

Abweichung zwischen den simulierten Temperaturbedingungen und dem Funktionsverlauf der Mittelwertfunktion, Cluster 10 


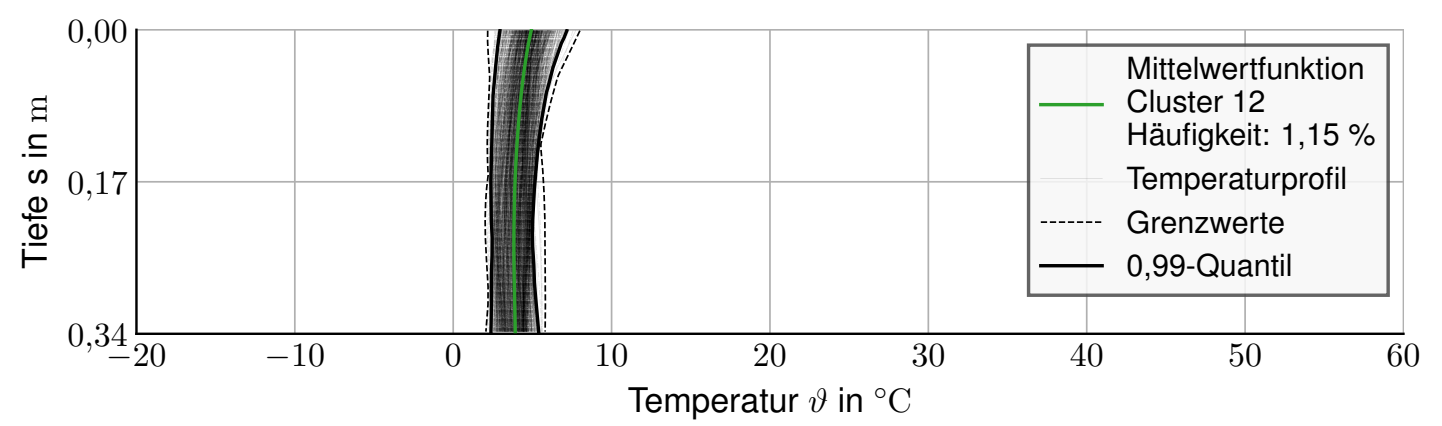

Abweichung zwischen den simulierten Temperaturbedingungen und dem Funktionsverlauf der Mittelwertfunktion, Cluster 11

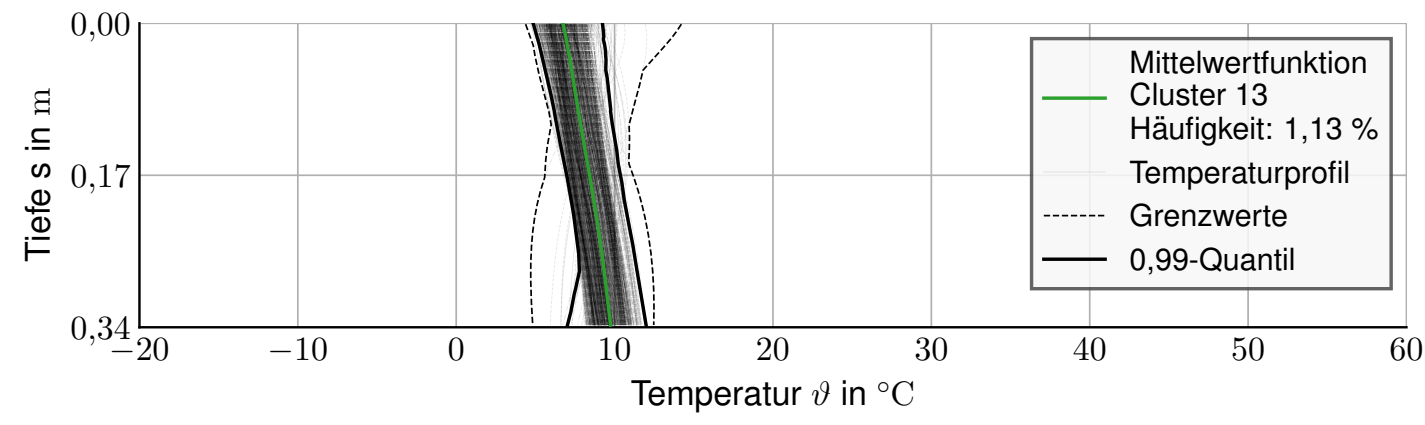

Abweichung zwischen den simulierten Temperaturbedingungen und dem Funktionsverlauf der Mittelwertfunktion, Cluster 12

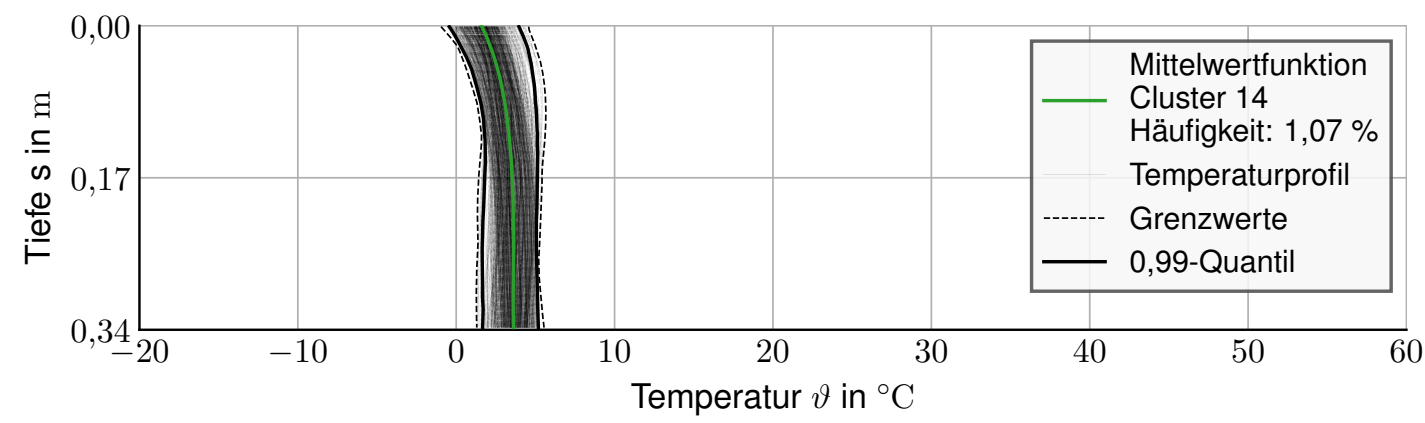

Abweichung zwischen den simulierten Temperaturbedingungen und dem Funktionsverlauf der Mittelwertfunktion, Cluster 13 


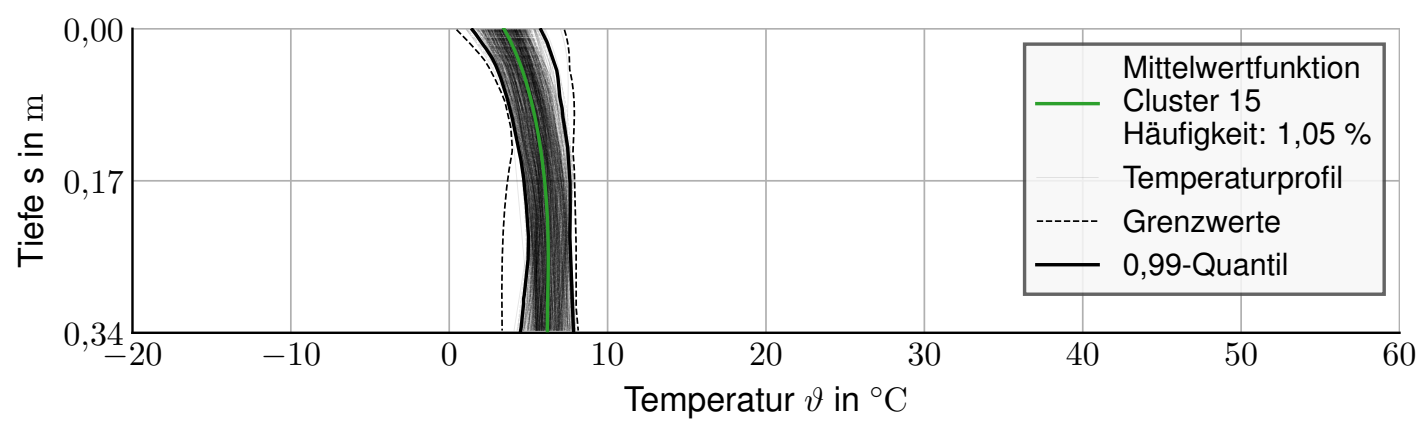

Abweichung zwischen den simulierten Temperaturbedingungen und dem Funktionsverlauf der Mittelwertfunktion, Cluster 14

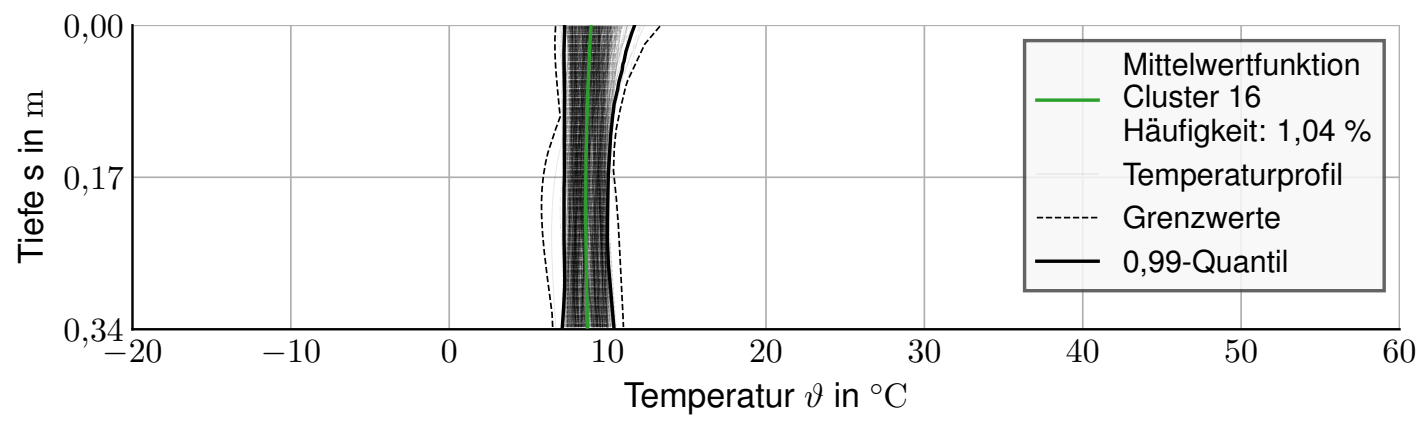

Abweichung zwischen den simulierten Temperaturbedingungen und dem Funktionsverlauf der Mittelwertfunktion, Cluster 15

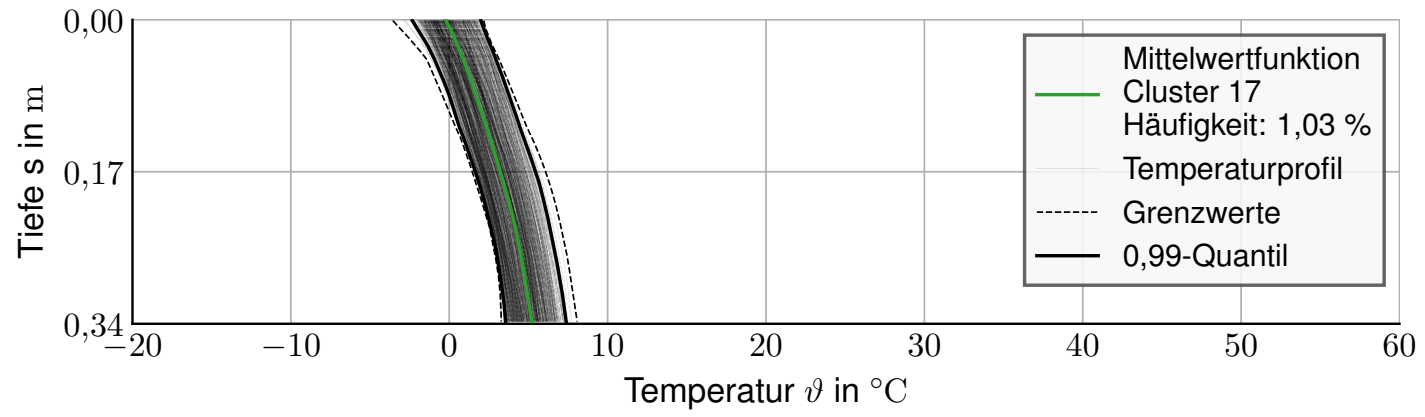

Abweichung zwischen den simulierten Temperaturbedingungen und dem Funktionsverlauf der Mittelwertfunktion, Cluster 16 


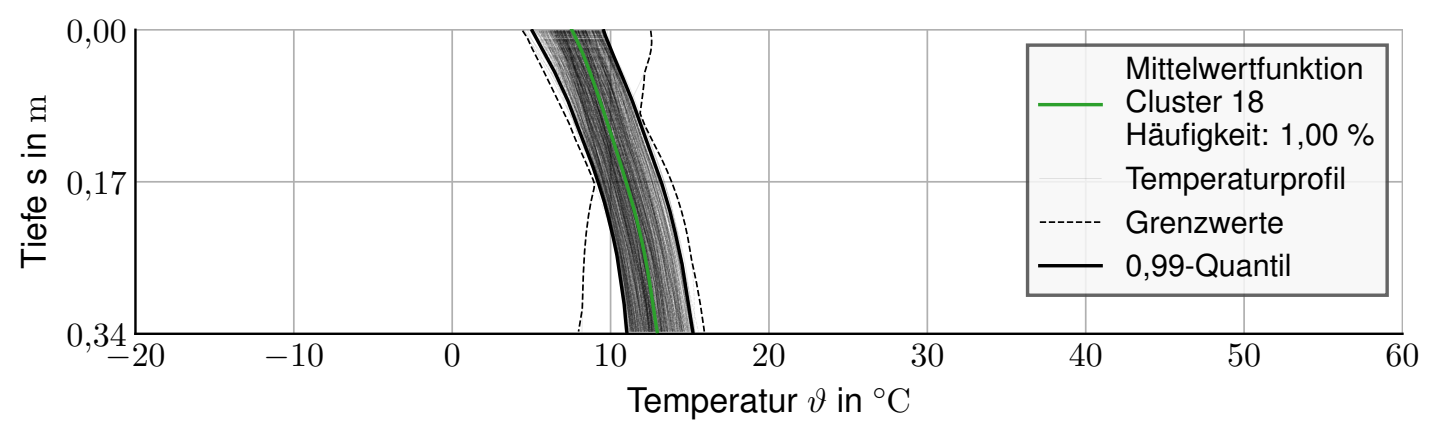

Abweichung zwischen den simulierten Temperaturbedingungen und dem Funktionsverlauf der Mittelwertfunktion, Cluster 17

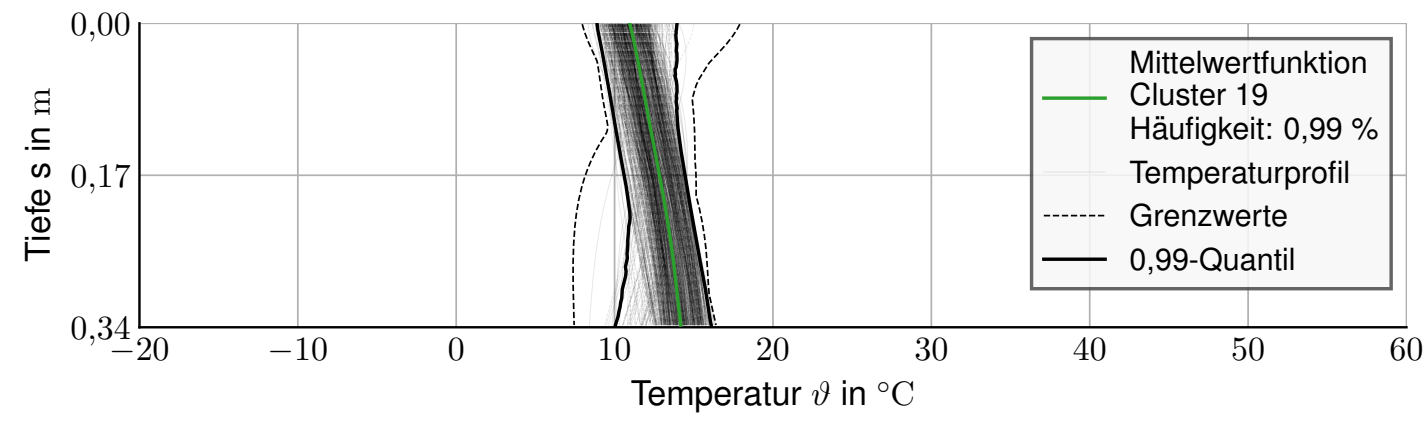

Abweichung zwischen den simulierten Temperaturbedingungen und dem Funktionsverlauf der Mittelwertfunktion, Cluster 18

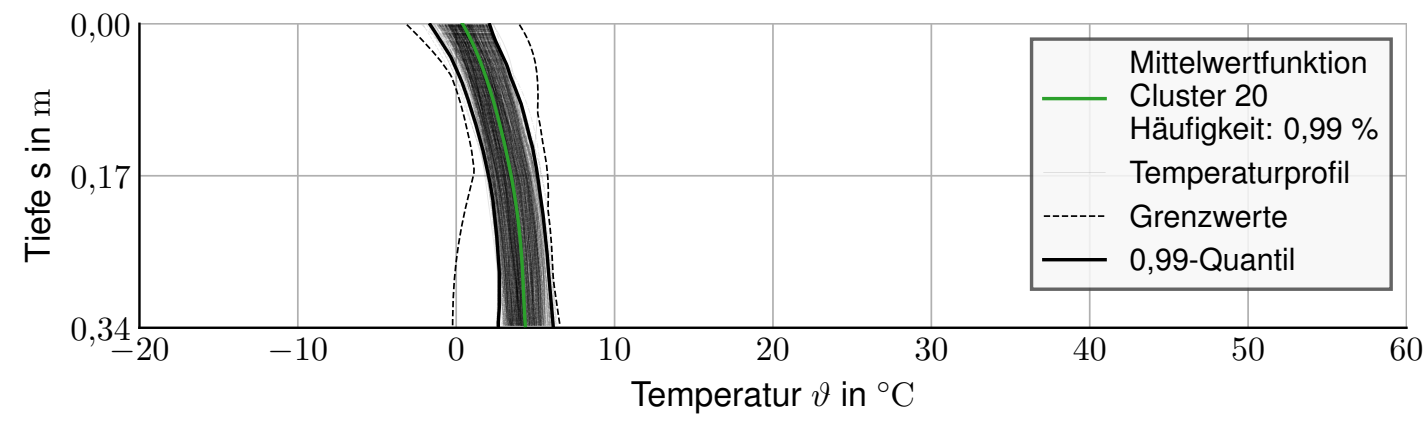

Abweichung zwischen den simulierten Temperaturbedingungen und dem Funktionsverlauf der Mittelwertfunktion, Cluster 19 


\section{B.2 Normierte charakteristische Temperaturprofile}

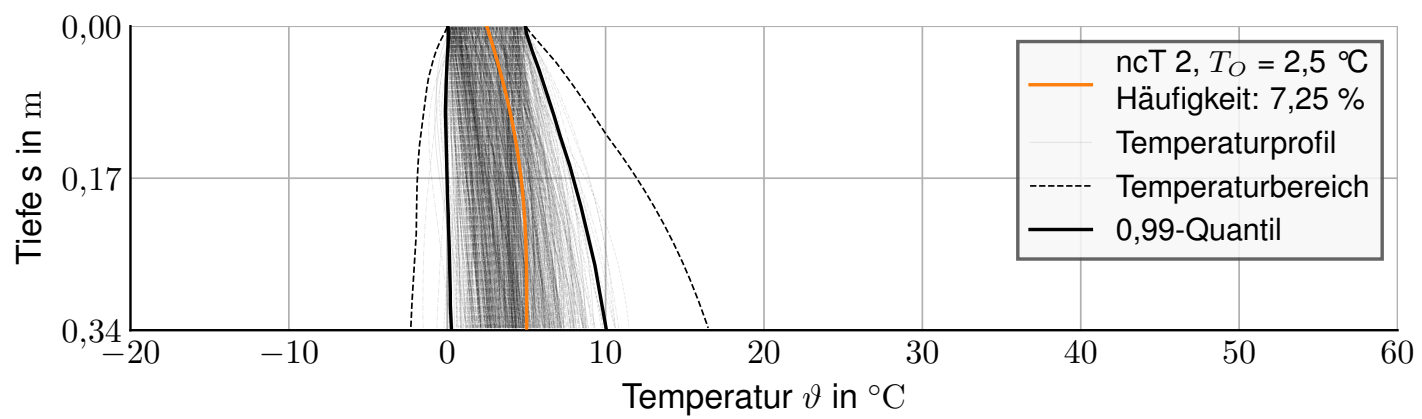

Abweichung zwischen den simulierten Temperaturbedingungen und dem Funktionsverlauf des charakteristischen normierten Temperaturprofiles

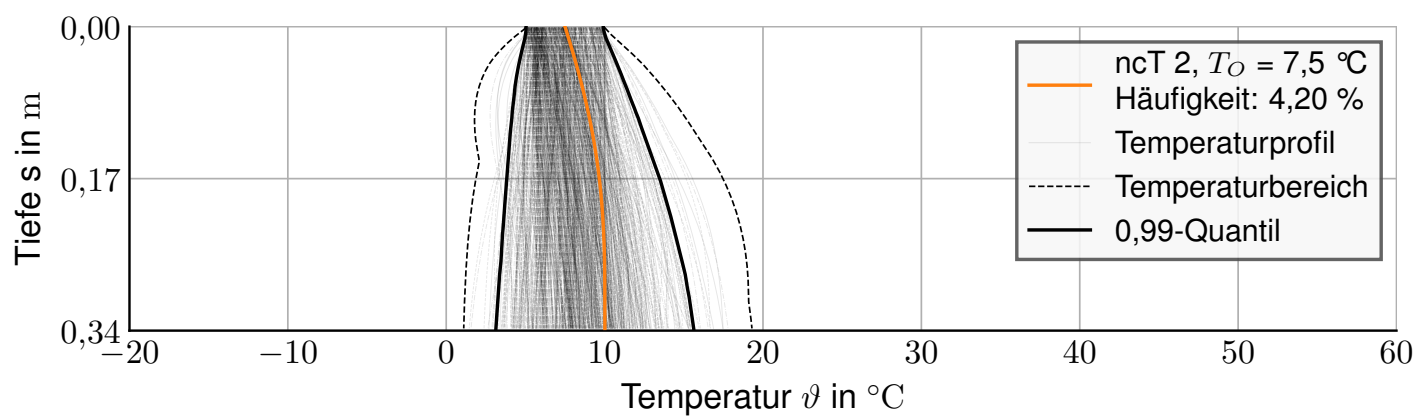

Abweichung zwischen den simulierten Temperaturbedingungen und dem Funktionsverlauf des charakteristischen normierten Temperaturprofiles

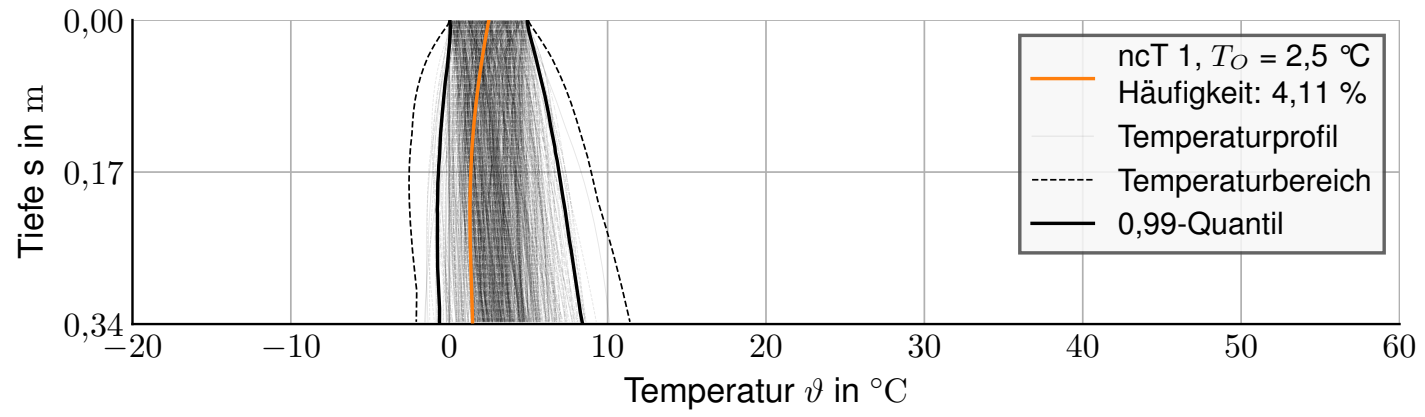

Abweichung zwischen den simulierten Temperaturbedingungen und dem Funktionsverlauf des charakteristischen normierten Temperaturprofiles 


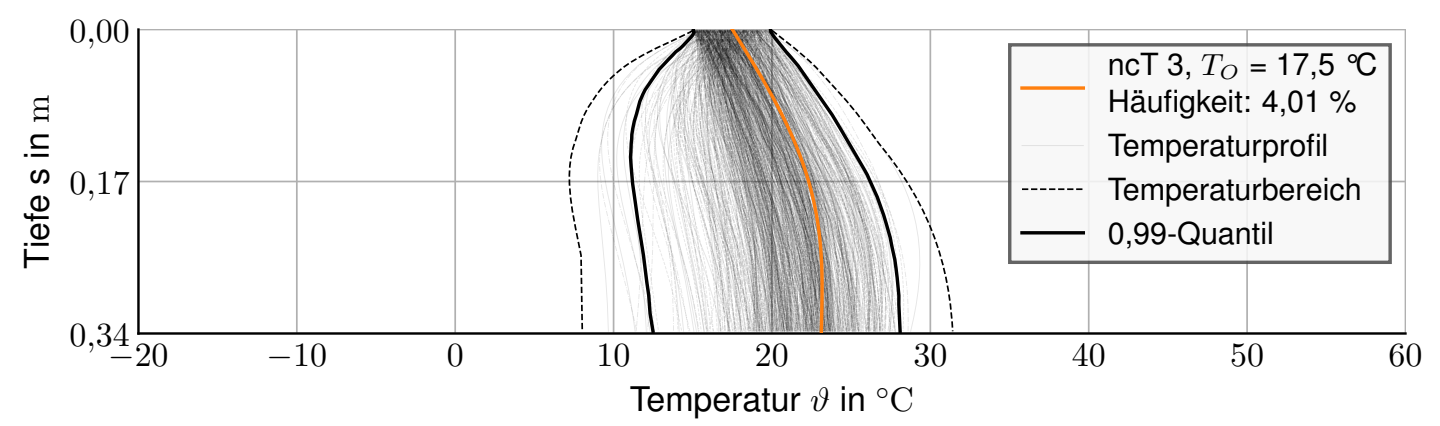

Abweichung zwischen den simulierten Temperaturbedingungen und dem Funktionsverlauf des charakteristischen normierten Temperaturprofiles

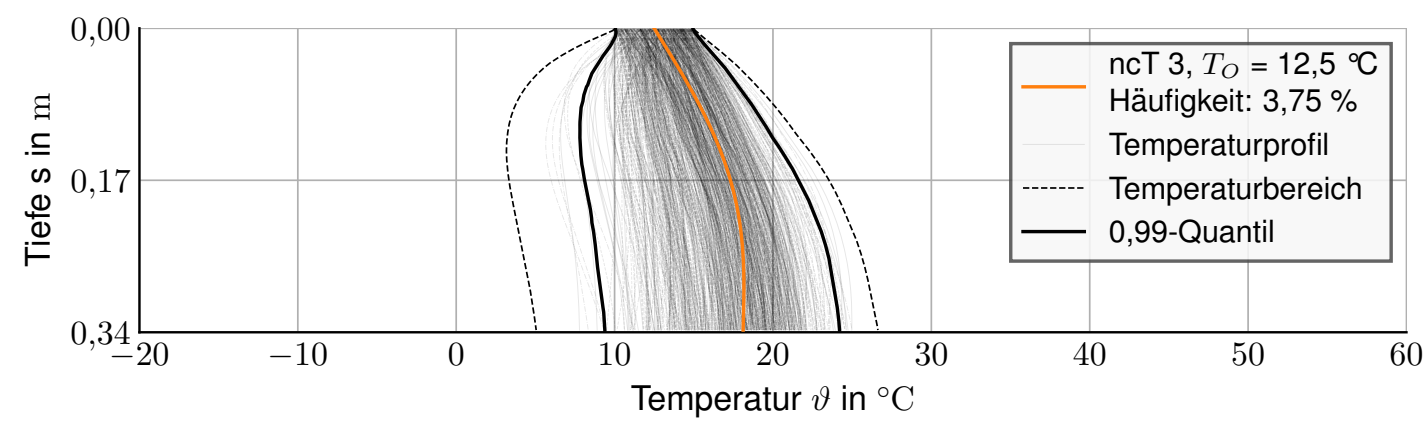

Abweichung zwischen den simulierten Temperaturbedingungen und dem Funktionsverlauf des charakteristischen normierten Temperaturprofiles

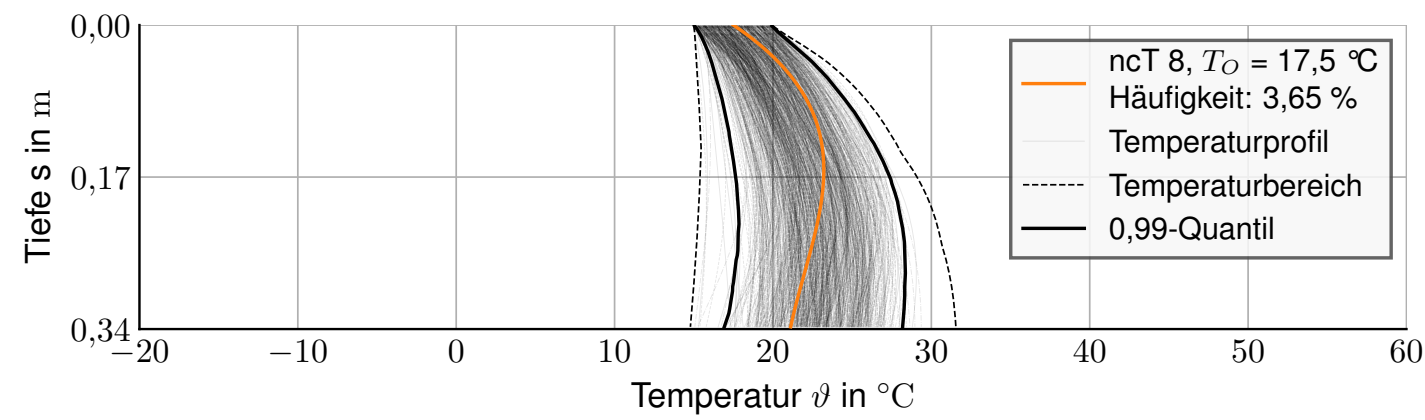

Abweichung zwischen den simulierten Temperaturbedingungen und dem Funktionsverlauf des charakteristischen normierten Temperaturprofiles 


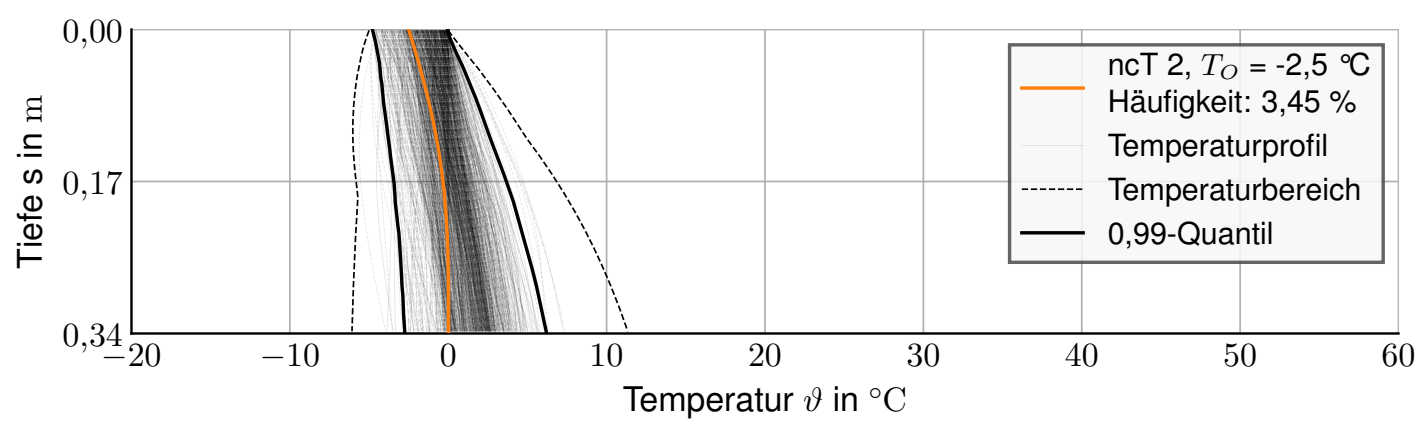

Abweichung zwischen den simulierten Temperaturbedingungen und dem Funktionsverlauf des charakteristischen normierten Temperaturprofiles

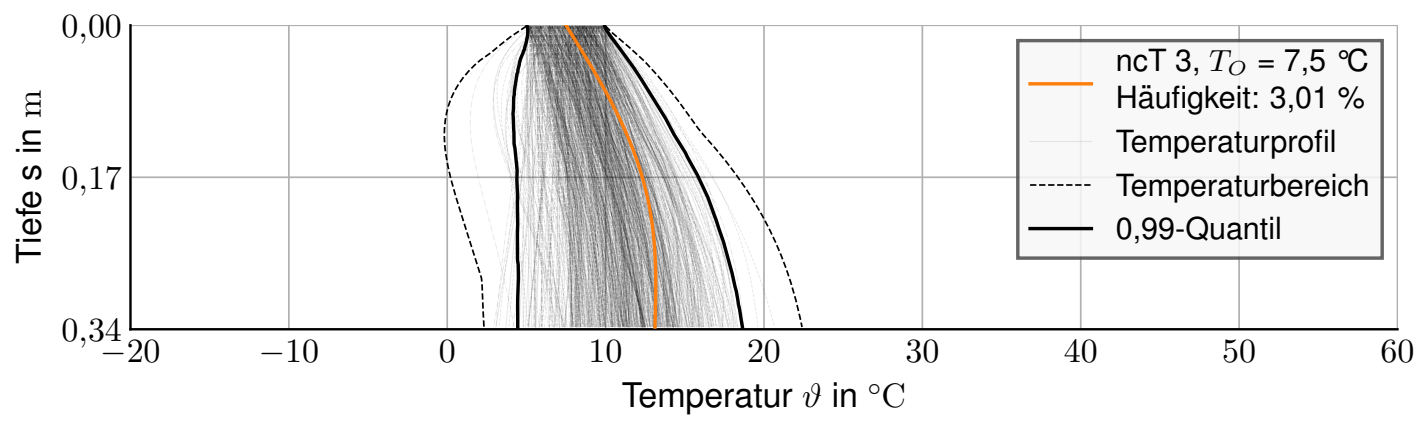

Abweichung zwischen den simulierten Temperaturbedingungen und dem Funktionsverlauf des charakteristischen normierten Temperaturprofiles

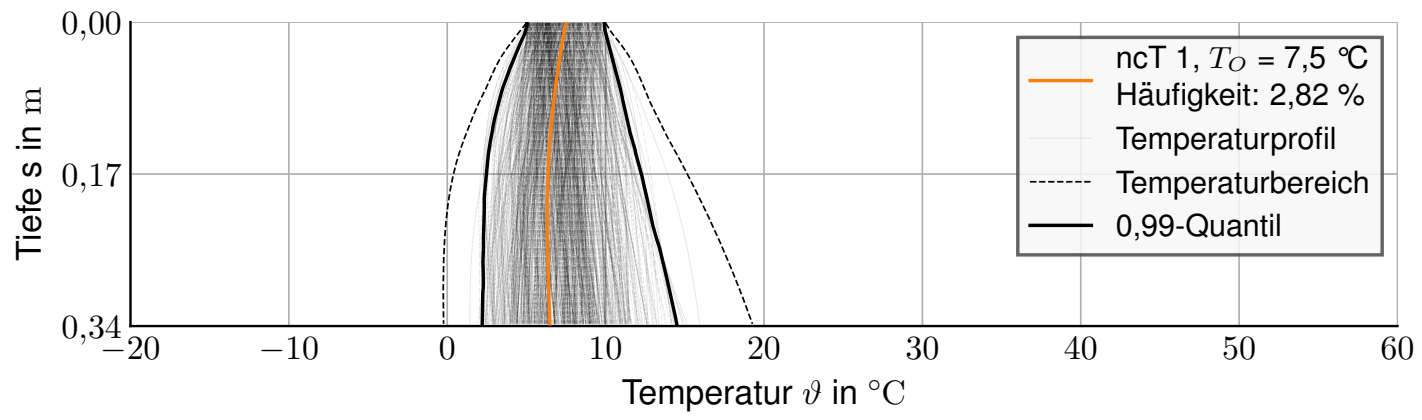

Abweichung zwischen den simulierten Temperaturbedingungen und dem Funktionsverlauf des charakteristischen normierten Temperaturprofiles 


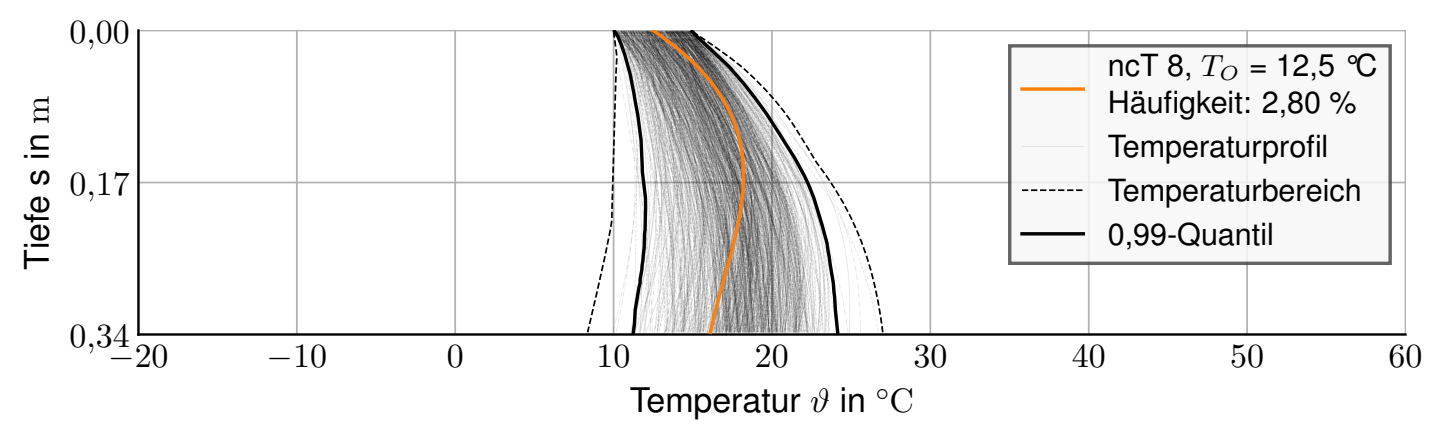

Abweichung zwischen den simulierten Temperaturbedingungen und dem Funktionsverlauf des charakteristischen normierten Temperaturprofiles

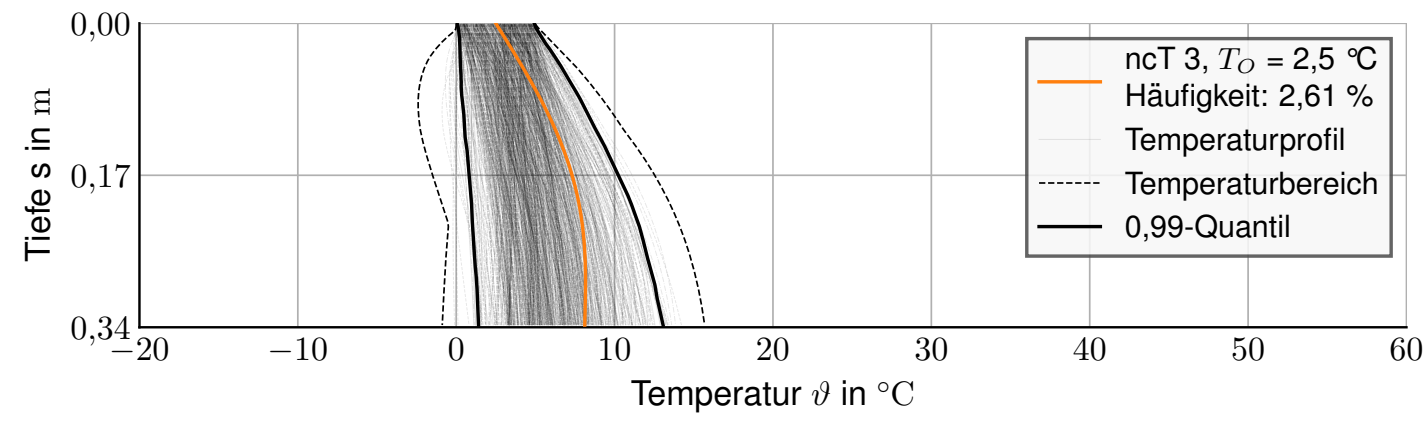

Abweichung zwischen den simulierten Temperaturbedingungen und dem Funktionsverlauf des charakteristischen normierten Temperaturprofiles

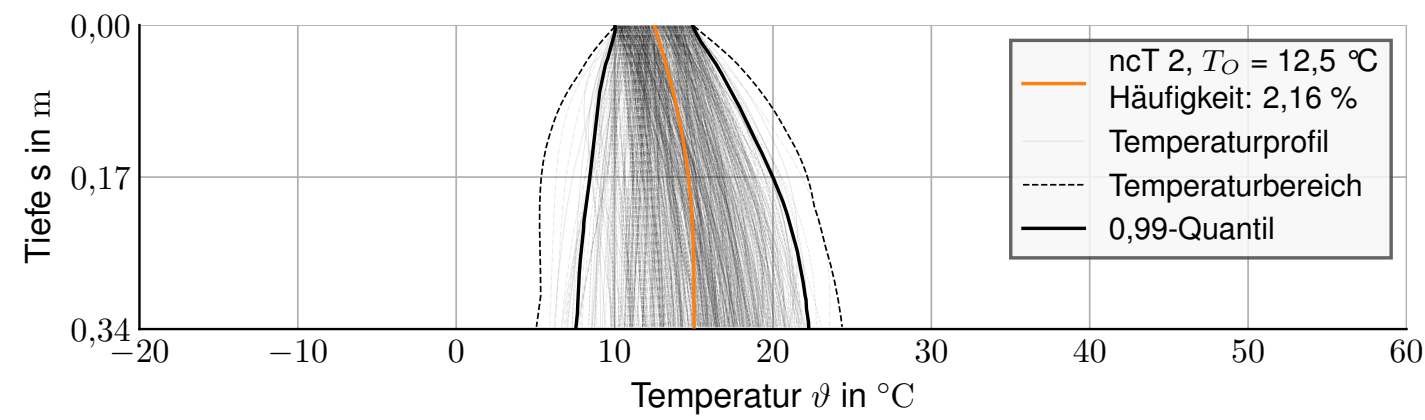

Abweichung zwischen den simulierten Temperaturbedingungen und dem Funktionsverlauf des charakteristischen normierten Temperaturprofiles 


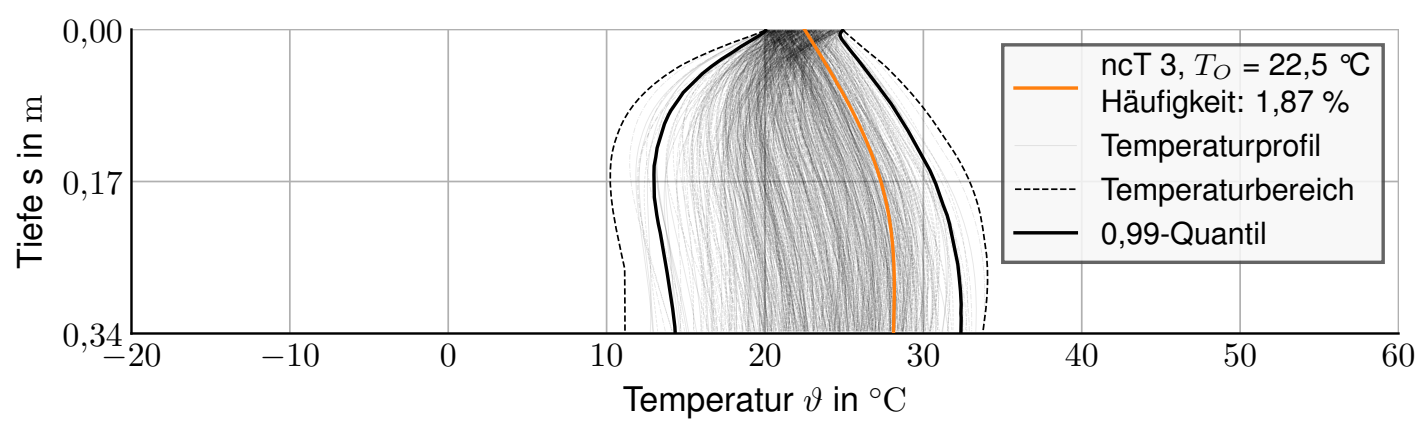

Abweichung zwischen den simulierten Temperaturbedingungen und dem Funktionsverlauf des charakteristischen normierten Temperaturprofiles

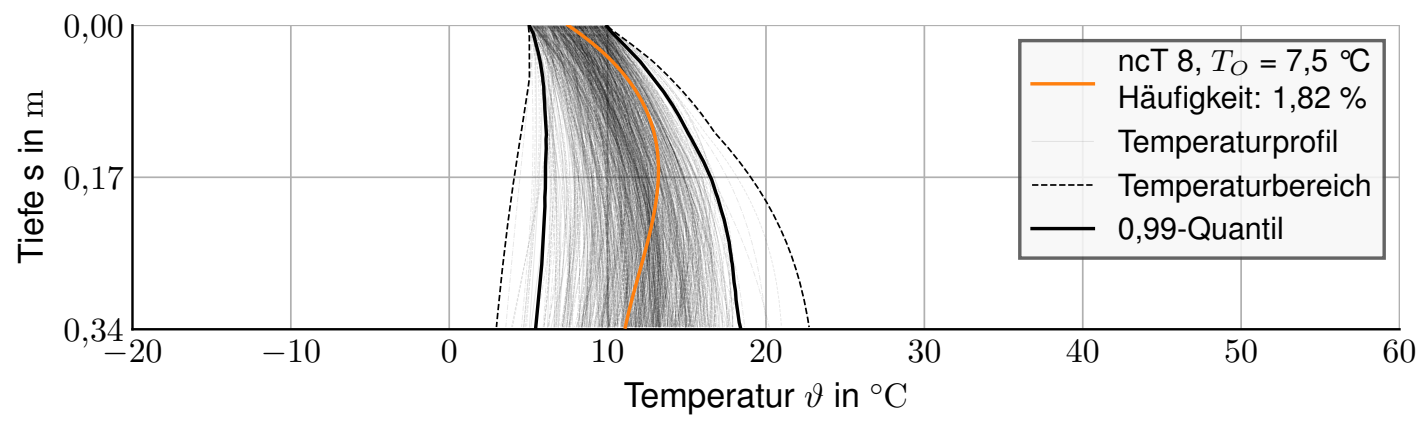

Abweichung zwischen den simulierten Temperaturbedingungen und dem Funktionsverlauf des charakteristischen normierten Temperaturprofiles

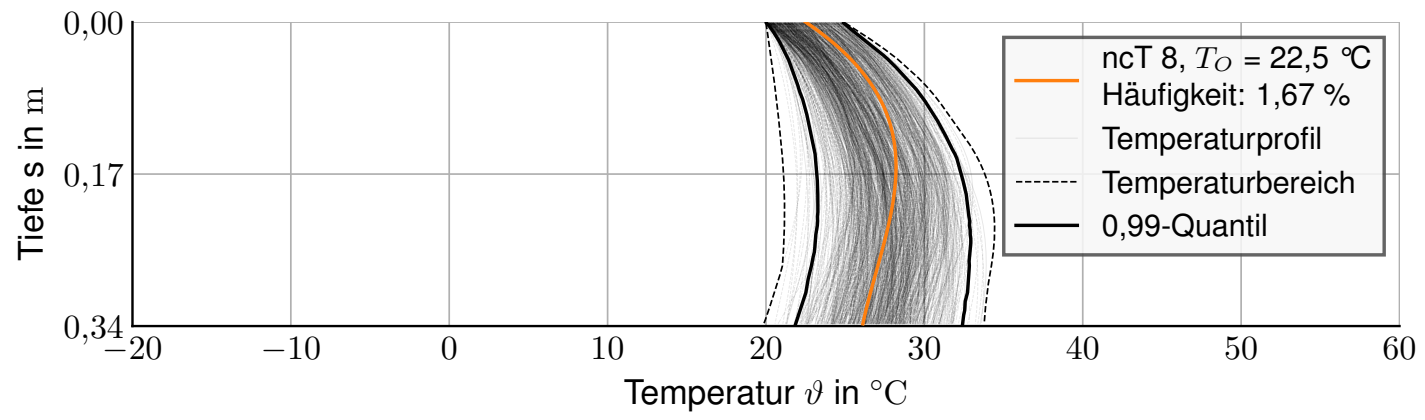

Abweichung zwischen den simulierten Temperaturbedingungen und dem Funktionsverlauf des charakteristischen normierten Temperaturprofiles 


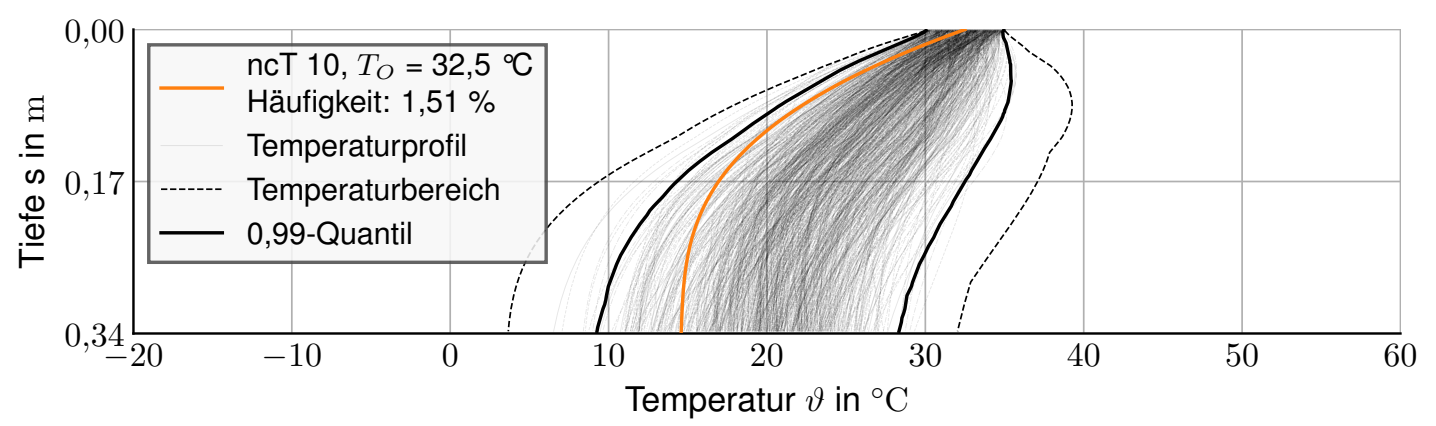

Abweichung zwischen den simulierten Temperaturbedingungen und dem Funktionsverlauf des charakteristischen normierten Temperaturprofiles

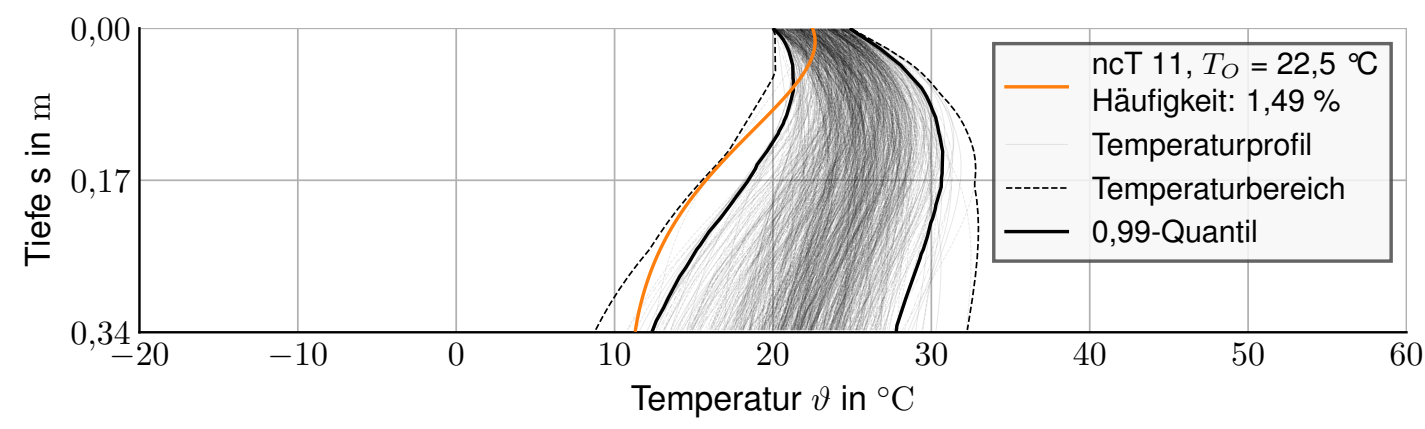

Abweichung zwischen den simulierten Temperaturbedingungen und dem Funktionsverlauf des charakteristischen normierten Temperaturprofiles

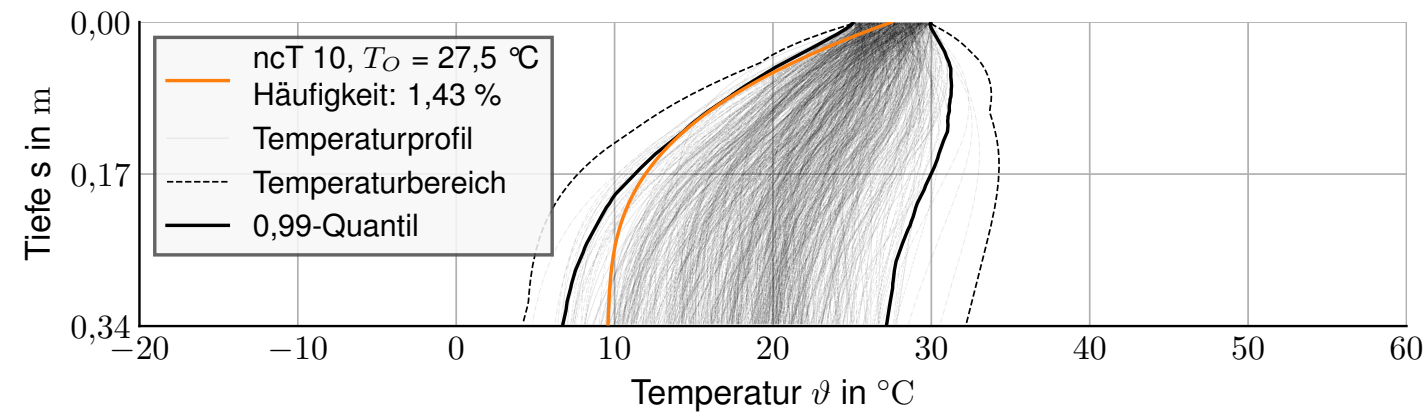

Abweichung zwischen den simulierten Temperaturbedingungen und dem Funktionsverlauf des charakteristischen normierten Temperaturprofiles 


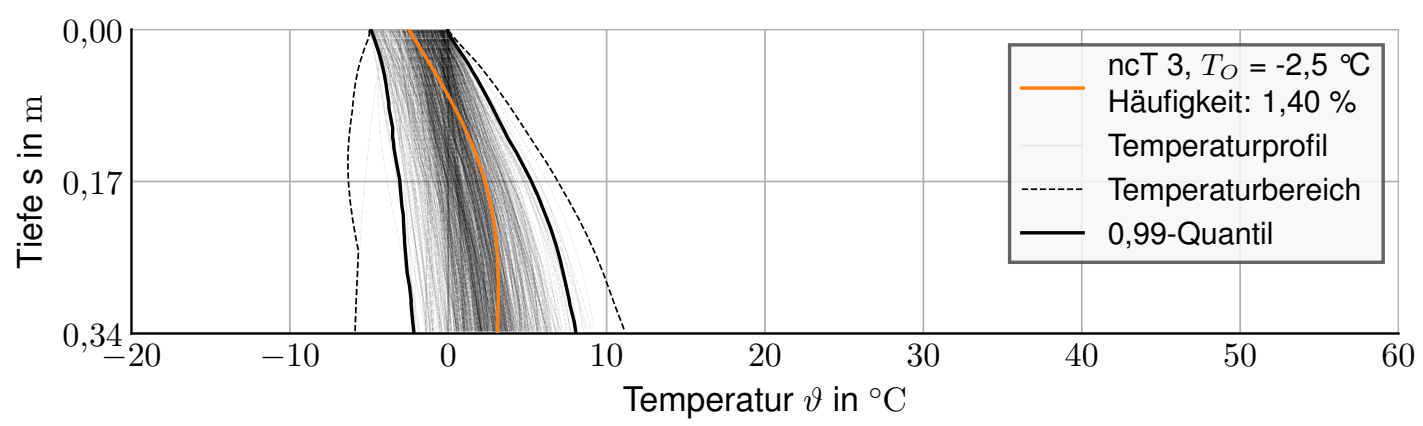

Abweichung zwischen den simulierten Temperaturbedingungen und dem Funktionsverlauf des charakteristischen normierten Temperaturprofiles

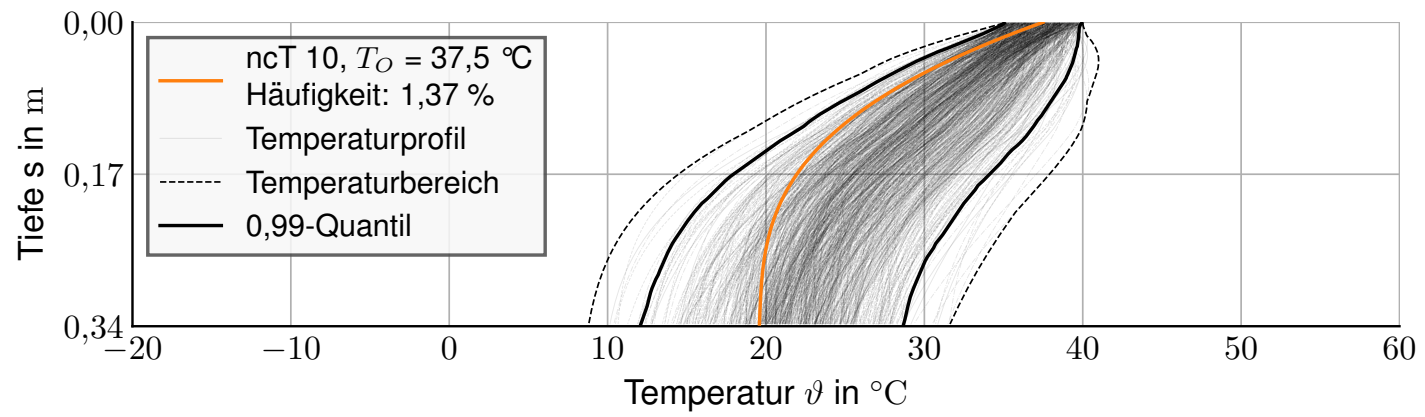

Abweichung zwischen den simulierten Temperaturbedingungen und dem Funktionsverlauf des charakteristischen normierten Temperaturprofiles 


\section{B.3 Temperaturprofile nach den RDO Asphalt}

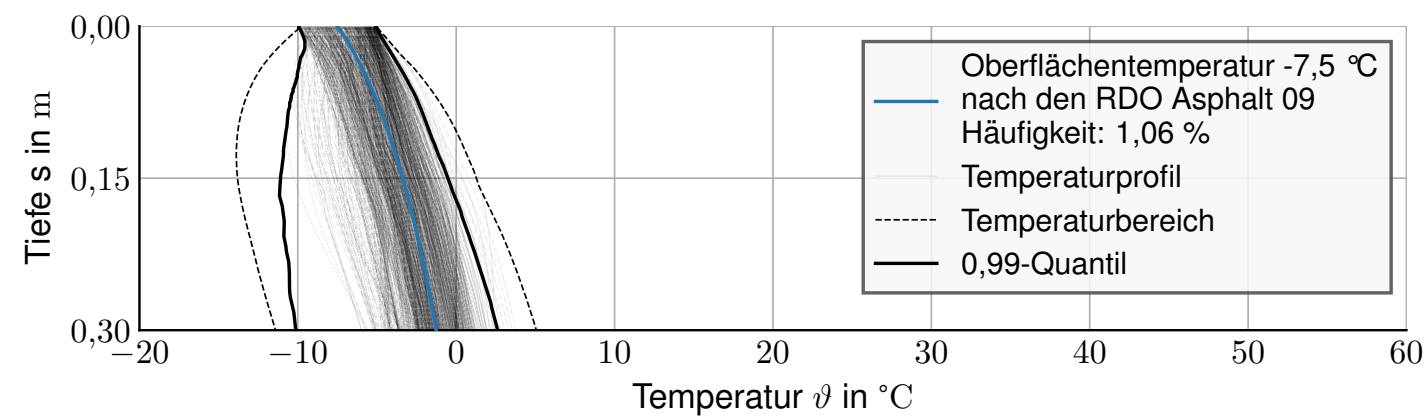

Abweichung zwischen den simulierten Temperaturbedingungen und dem Funktionsverlauf des Temperaturprofiles nach den RDO Asphalt, Temperaturklasse 1

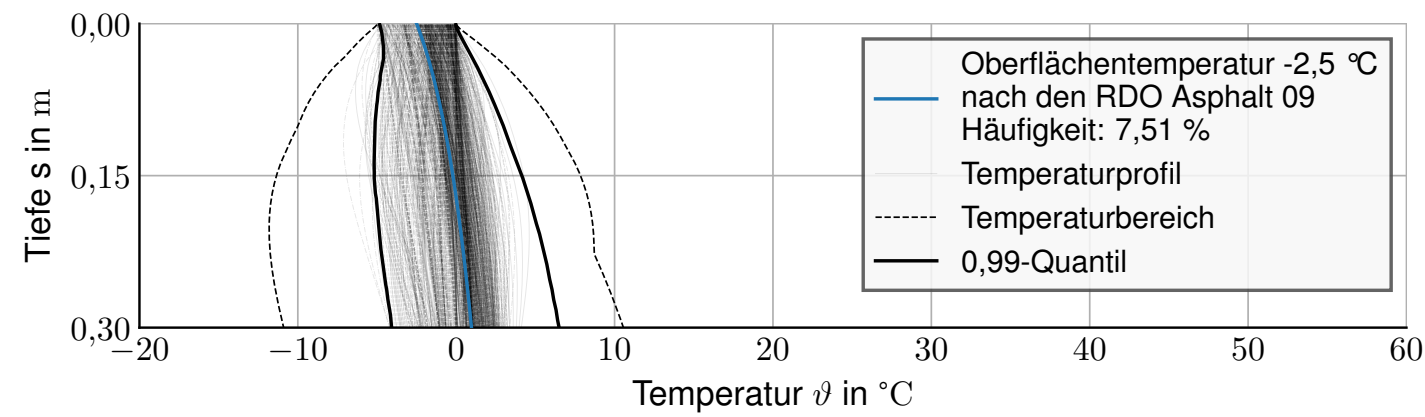

Abweichung zwischen den simulierten Temperaturbedingungen und dem Funktionsverlauf des Temperaturprofiles nach den RDO Asphalt, Temperaturklasse 2

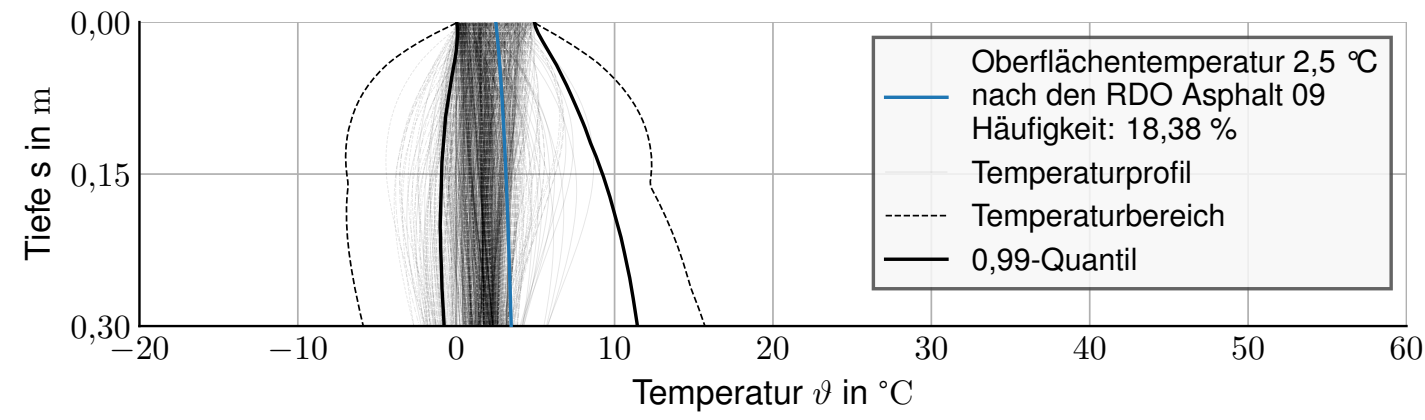

Abweichung zwischen den simulierten Temperaturbedingungen und dem Funktionsverlauf des Temperaturprofiles nach den RDO Asphalt, Temperaturklasse 3 


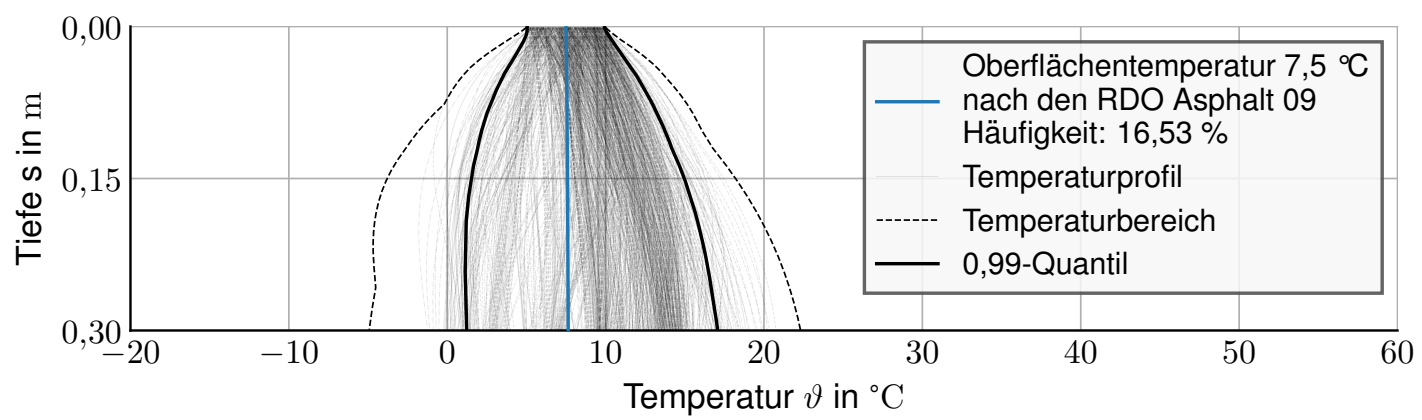

Abweichung zwischen den simulierten Temperaturbedingungen und dem Funktionsverlauf des Temperaturprofiles nach den RDO Asphalt, Temperaturklasse 4

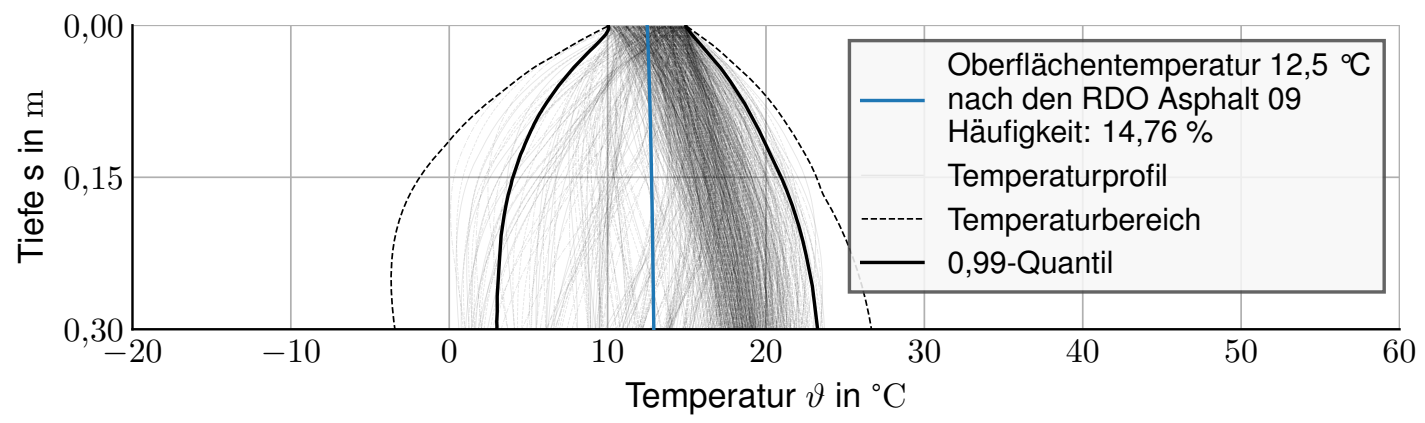

Abweichung zwischen den simulierten Temperaturbedingungen und dem Funktionsverlauf des Temperaturprofiles nach den RDO Asphalt, Temperaturklasse 5

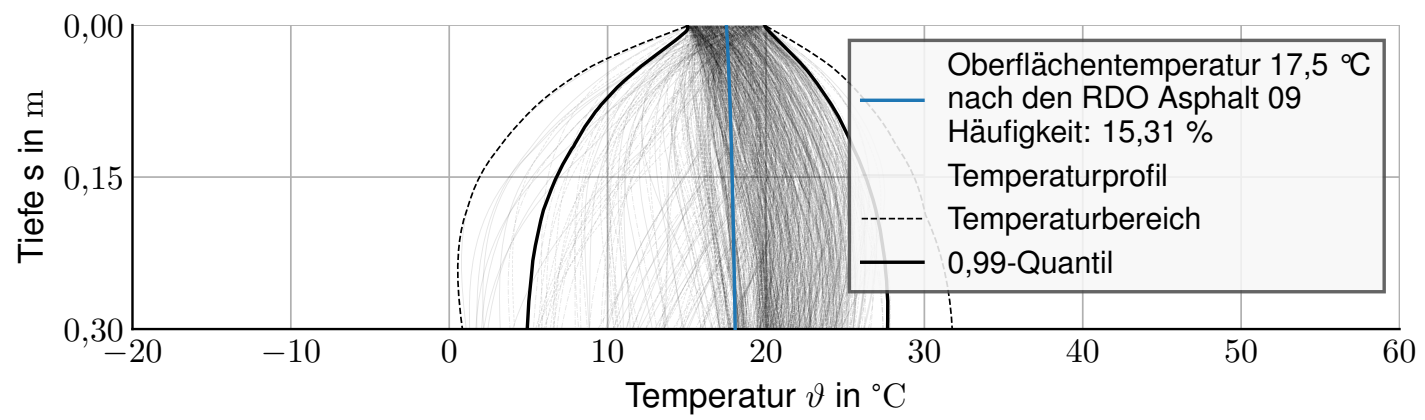

Abweichung zwischen den simulierten Temperaturbedingungen und dem Funktionsverlauf des Temperaturprofiles nach den RDO Asphalt, Temperaturklasse 6 


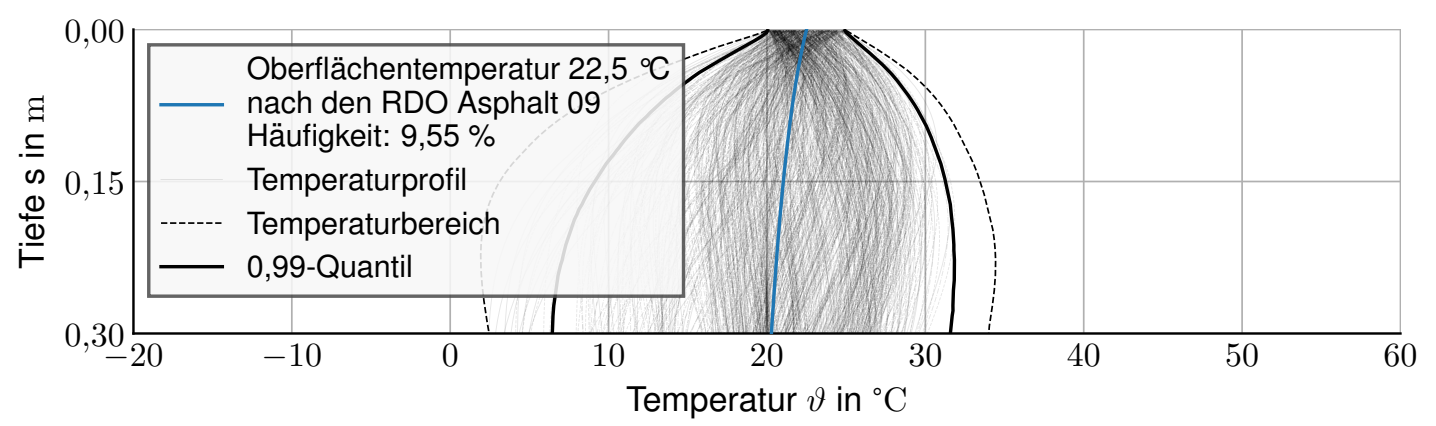

Abweichung zwischen den simulierten Temperaturbedingungen und dem Funktionsverlauf des Temperaturprofiles nach den RDO Asphalt, Temperaturklasse 7

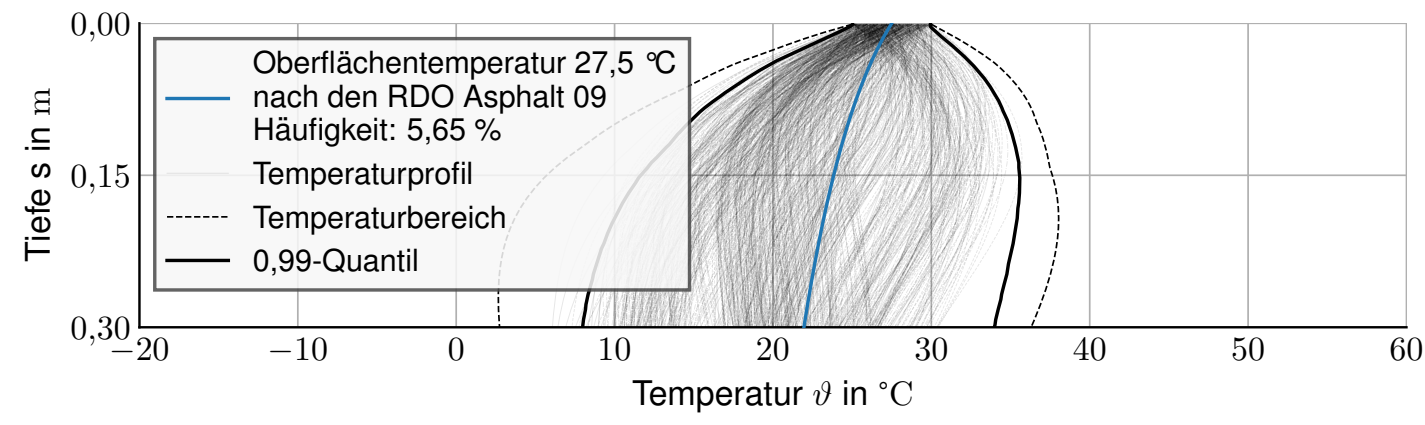

Abweichung zwischen den simulierten Temperaturbedingungen und dem Funktionsverlauf des Temperaturprofiles nach den RDO Asphalt, Temperaturklasse 8

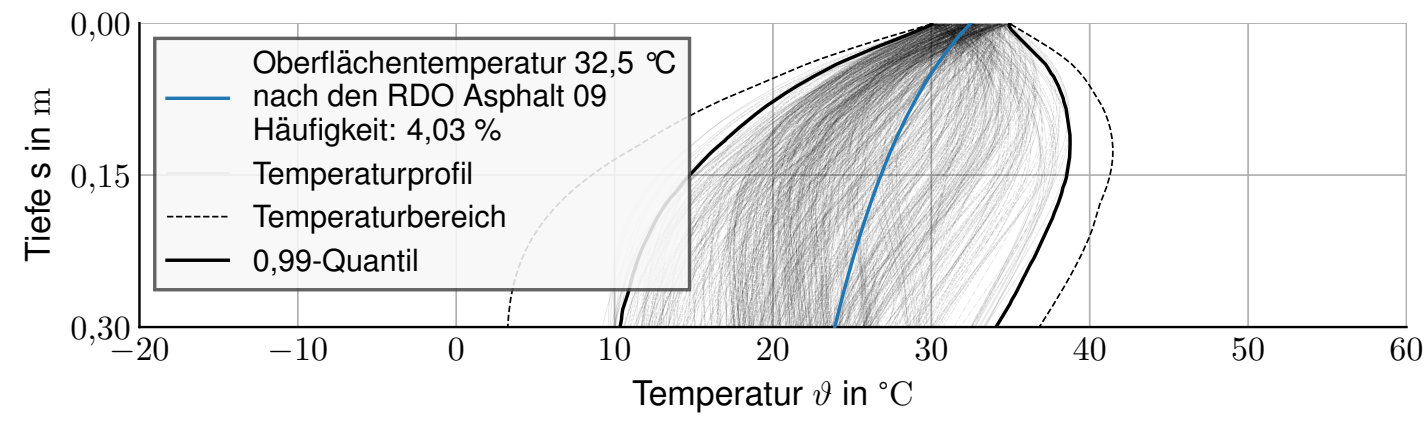

Abweichung zwischen den simulierten Temperaturbedingungen und dem Funktionsverlauf des Temperaturprofiles nach den RDO Asphalt, Temperaturklasse 9 


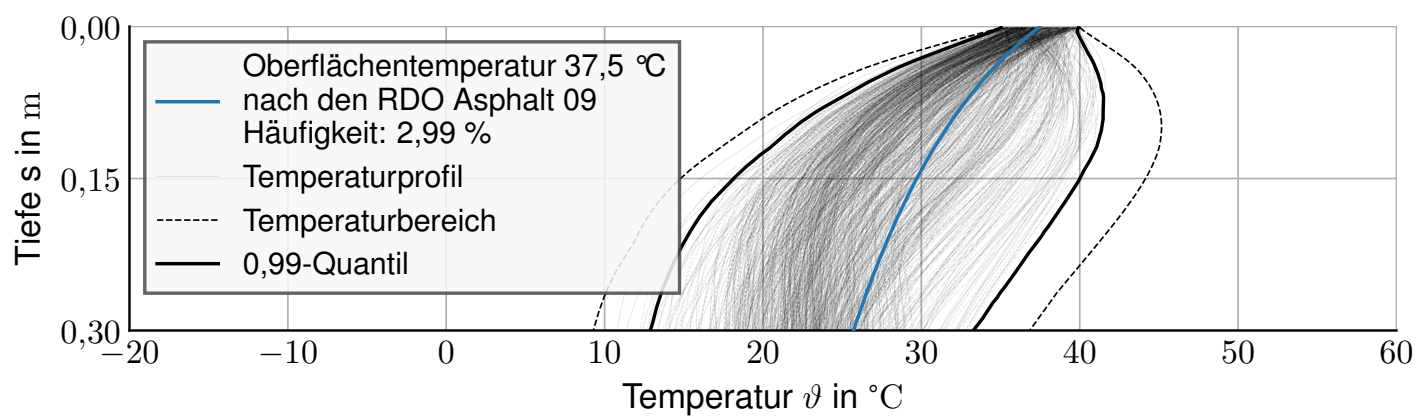

Abweichung zwischen den simulierten Temperaturbedingungen und dem Funktionsverlauf des Temperaturprofiles nach den RDO Asphalt, Temperaturklasse 10

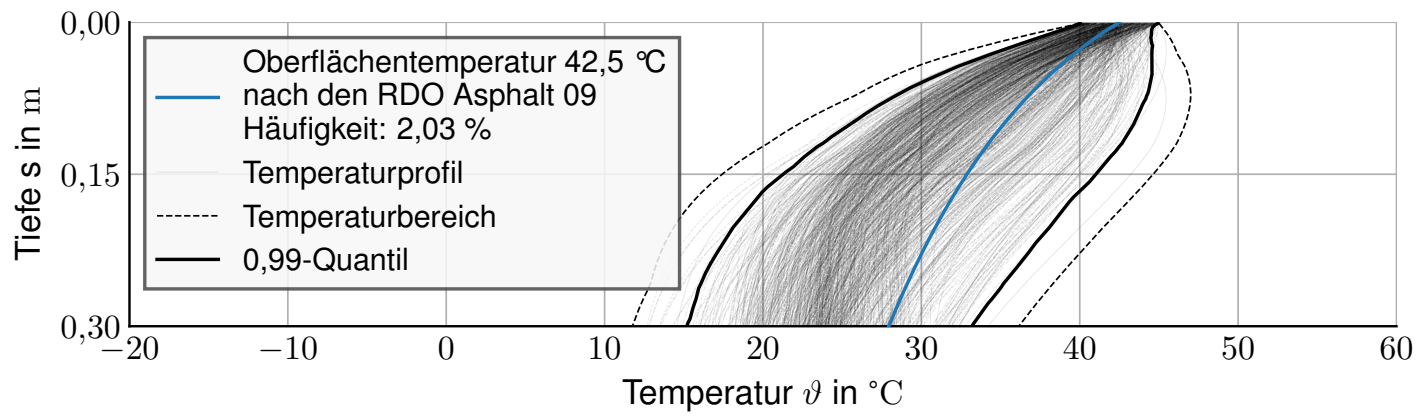

Abweichung zwischen den simulierten Temperaturbedingungen und dem Funktionsverlauf des Temperaturprofiles nach den RDO Asphalt, Temperaturklasse 11

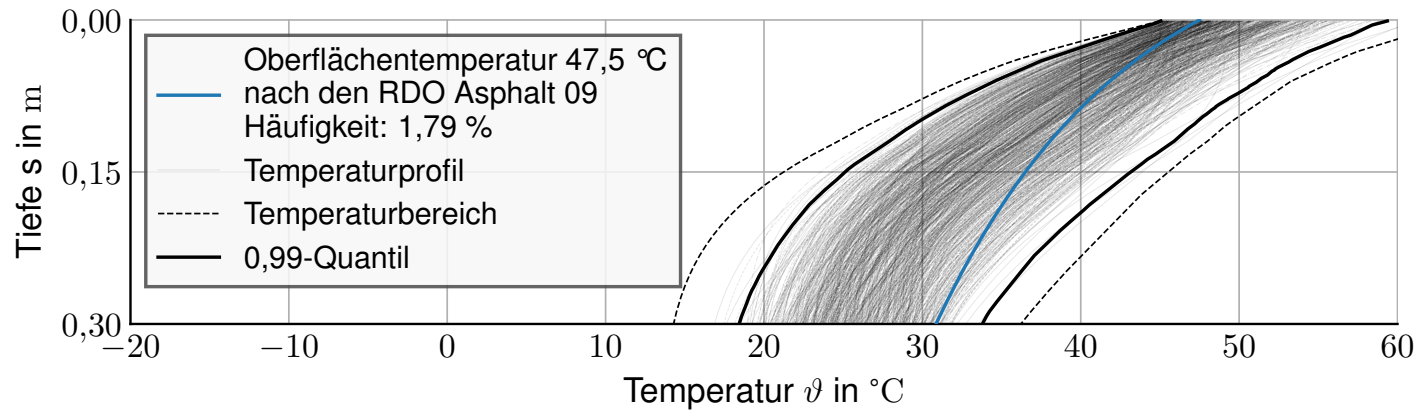

Abweichung zwischen den simulierten Temperaturbedingungen und dem Funktionsverlauf des Temperaturprofiles nach den RDO Asphalt, Temperaturklasse 12 


\section{B.4 Dimensionierung}


Anhang

Tabelle B.1

Relative Häufigkeitsverteilung der Fahrzeuge in Abhängigkeit der Tageszeit

\begin{tabular}{lcccc}
\hline & \multicolumn{2}{c}{ KFZ } & \multicolumn{2}{c}{ Schwerverkehr } \\
Tageszeit & Bundesautobahn & Bundesstraße & Bundesautobahn & Bundesstraße \\
\hline 1 & & & & \\
2 & 1,087 & 0,835 & 2,035 & 1,331 \\
3 & 0,819 & 0,581 & 2,021 & 1,313 \\
4 & 0,698 & 0,464 & 2,077 & 1,402 \\
5 & 0,747 & 0,493 & 2,375 & 1,728 \\
6 & 1,081 & 0,827 & 3,040 & 2,433 \\
7 & 2,257 & 2,218 & 4,127 & 3,704 \\
8 & 4,171 & 4,367 & 5,157 & 5,340 \\
9 & 5,635 & 5,869 & 5,444 & 6,173 \\
10 & 5,625 & 5,469 & 5,733 & 6,725 \\
11 & 5,422 & 5,412 & 5,869 & 6,833 \\
12 & 5,525 & 5,657 & 5,877 & 6,839 \\
13 & 5,754 & 5,868 & 5,889 & 6,809 \\
14 & 6,009 & 6,147 & 5,808 & 6,573 \\
15 & 6,352 & 6,587 & 5,777 & 6,602 \\
16 & 6,630 & 6,962 & 5,748 & 6,452 \\
17 & 6,871 & 7,237 & 5,546 & 6,035 \\
18 & 7,261 & 7,753 & 5,159 & 5,391 \\
19 & 7,109 & 7,433 & 4,577 & 4,429 \\
20 & 6,233 & 6,281 & 3,985 & 3,554 \\
21 & 4,776 & 4,596 & 3,446 & 2,842 \\
22 & 3,542 & 3,259 & 3,002 & 2,319 \\
23 & 2,682 & 2,423 & 2,633 & 1,932 \\
\hline & 2,159 & 1,961 & 2,465 & 1,748 \\
\hline
\end{tabular}




\section{Dimensionierungsberechnungen}

C.1 Miner-Summe 


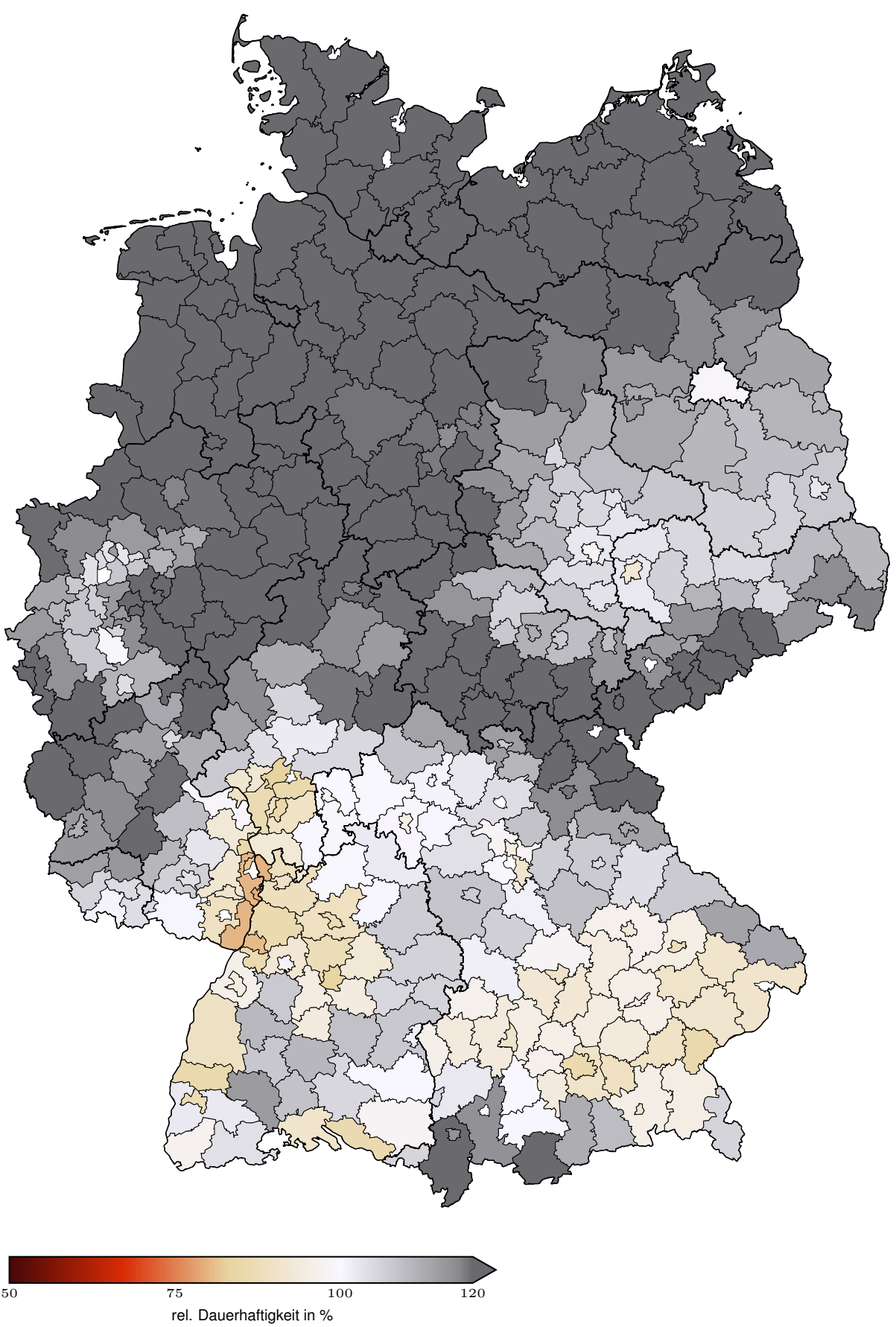

Relative Dauerhaftigkeit für das Gebiet Deutschland auf Basis der Mittelwertfunktionen, normiert auf den Mittelwert aller Landkreise mit der Verkehrsfreigabe 2020, Verkehrsfreigabe 1960 


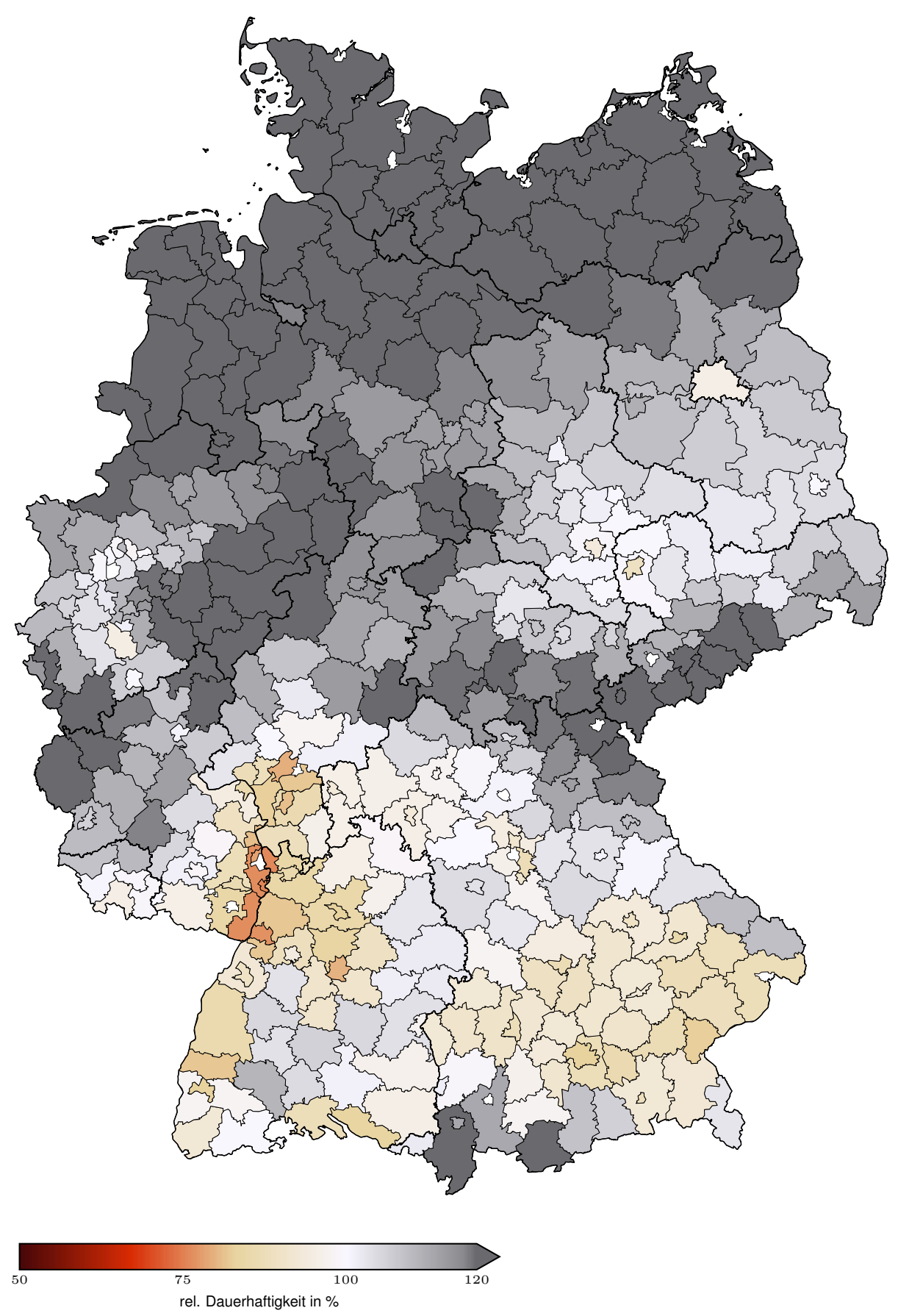

Relative Dauerhaftigkeit für das Gebiet Deutschland auf Basis der Mittelwertfunktionen, normiert auf den Mittelwert aller Landkreise mit der Verkehrsfreigabe 2020, Verkehrsfreigabe 1970 


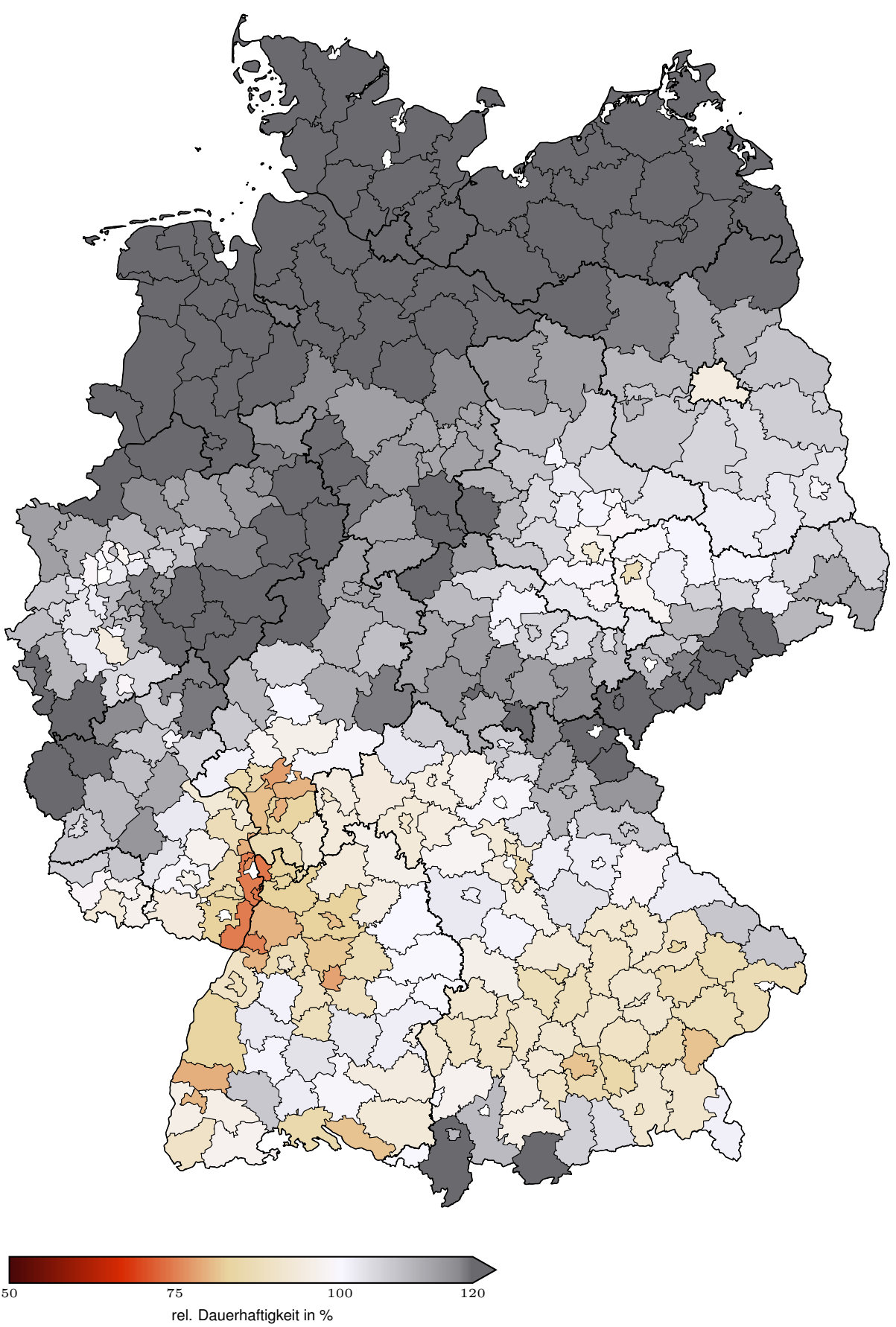

Relative Dauerhaftigkeit für das Gebiet Deutschland auf Basis der Mittelwertfunktionen, normiert auf den Mittelwert aller Landkreise mit der Verkehrsfreigabe 2020, Verkehrsfreigabe 1980 


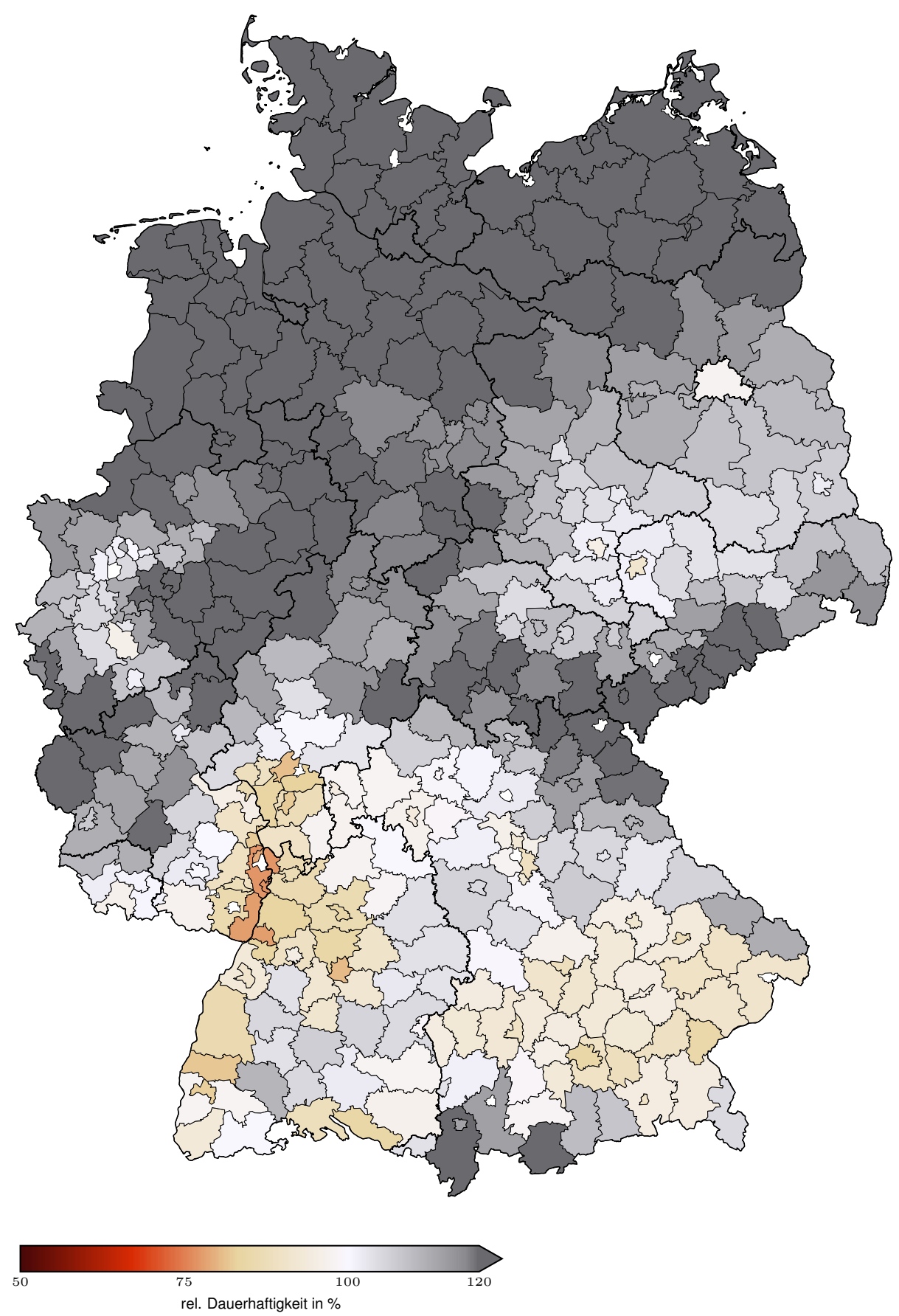

Relative Dauerhaftigkeit für das Gebiet Deutschland auf Basis der Mittelwertfunktionen, normiert auf den Mittelwert aller Landkreise mit der Verkehrsfreigabe 2020, Verkehrsfreigabe 1990 


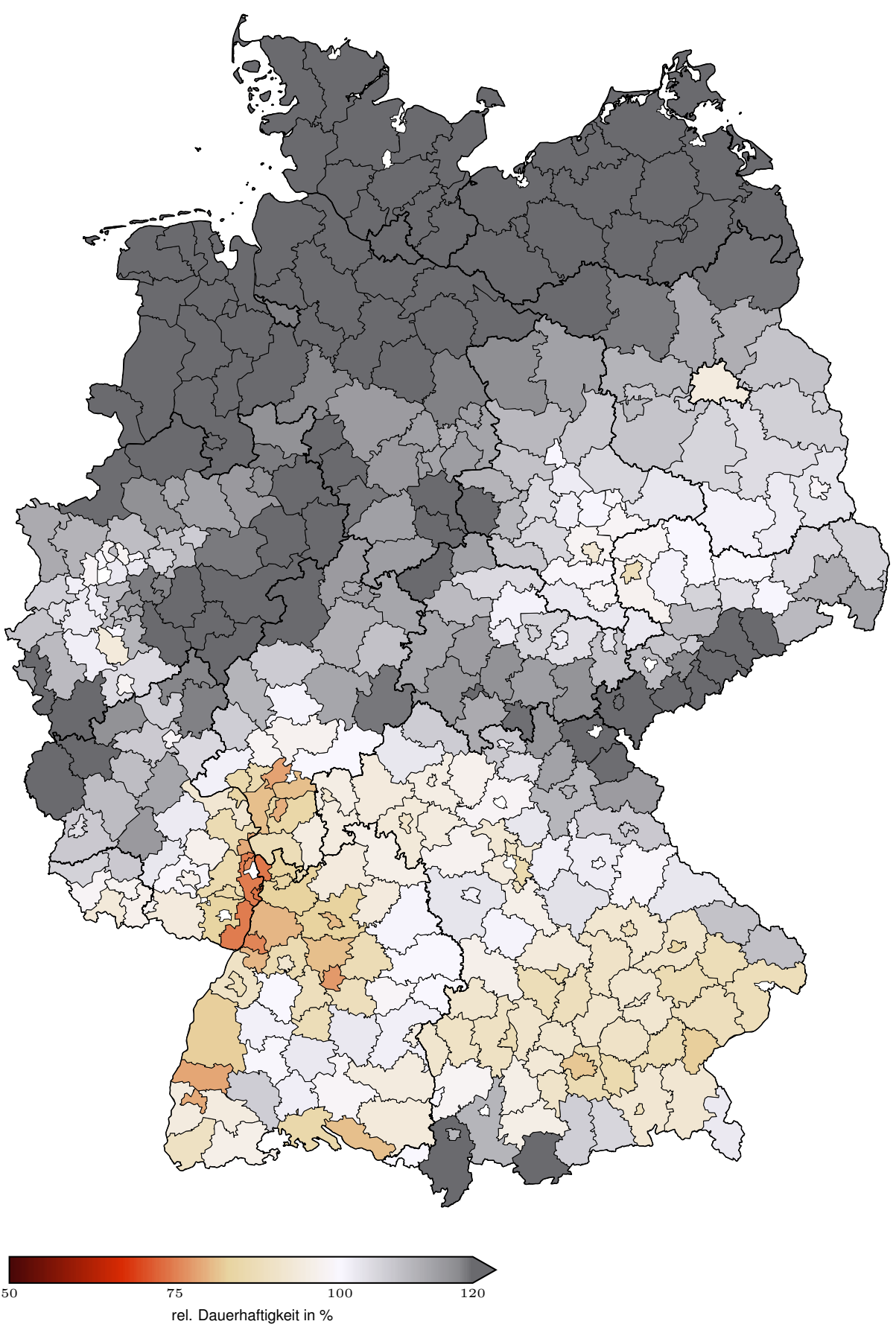

Relative Dauerhaftigkeit für das Gebiet Deutschland auf Basis der Mittelwertfunktionen, normiert auf den Mittelwert aller Landkreise mit der Verkehrsfreigabe 2020, Verkehrsfreigabe 2000 


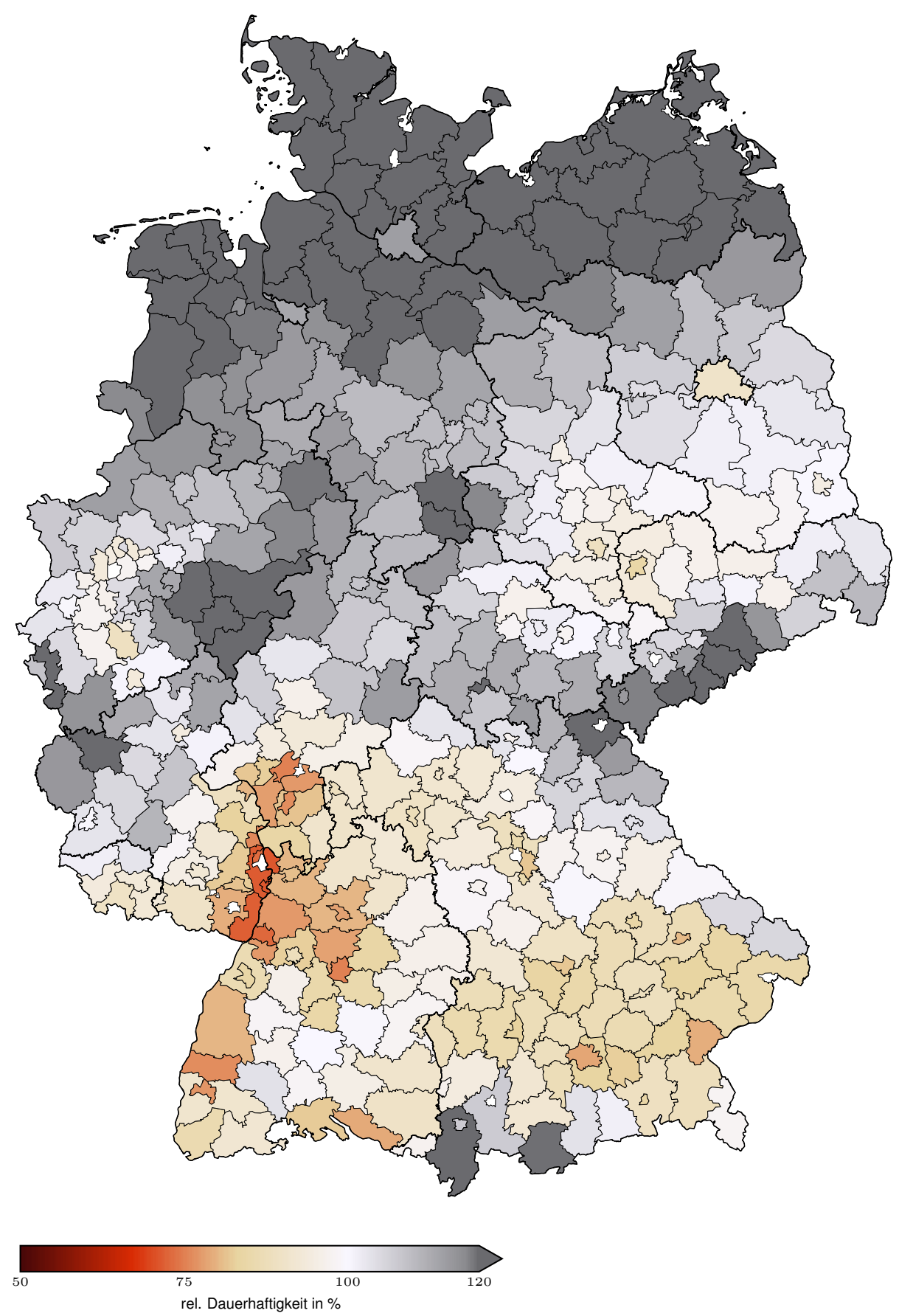

Relative Dauerhaftigkeit für das Gebiet Deutschland auf Basis der Mittelwertfunktionen, normiert auf den Mittelwert aller Landkreise mit der Verkehrsfreigabe 2020, Verkehrsfreigabe 2010 


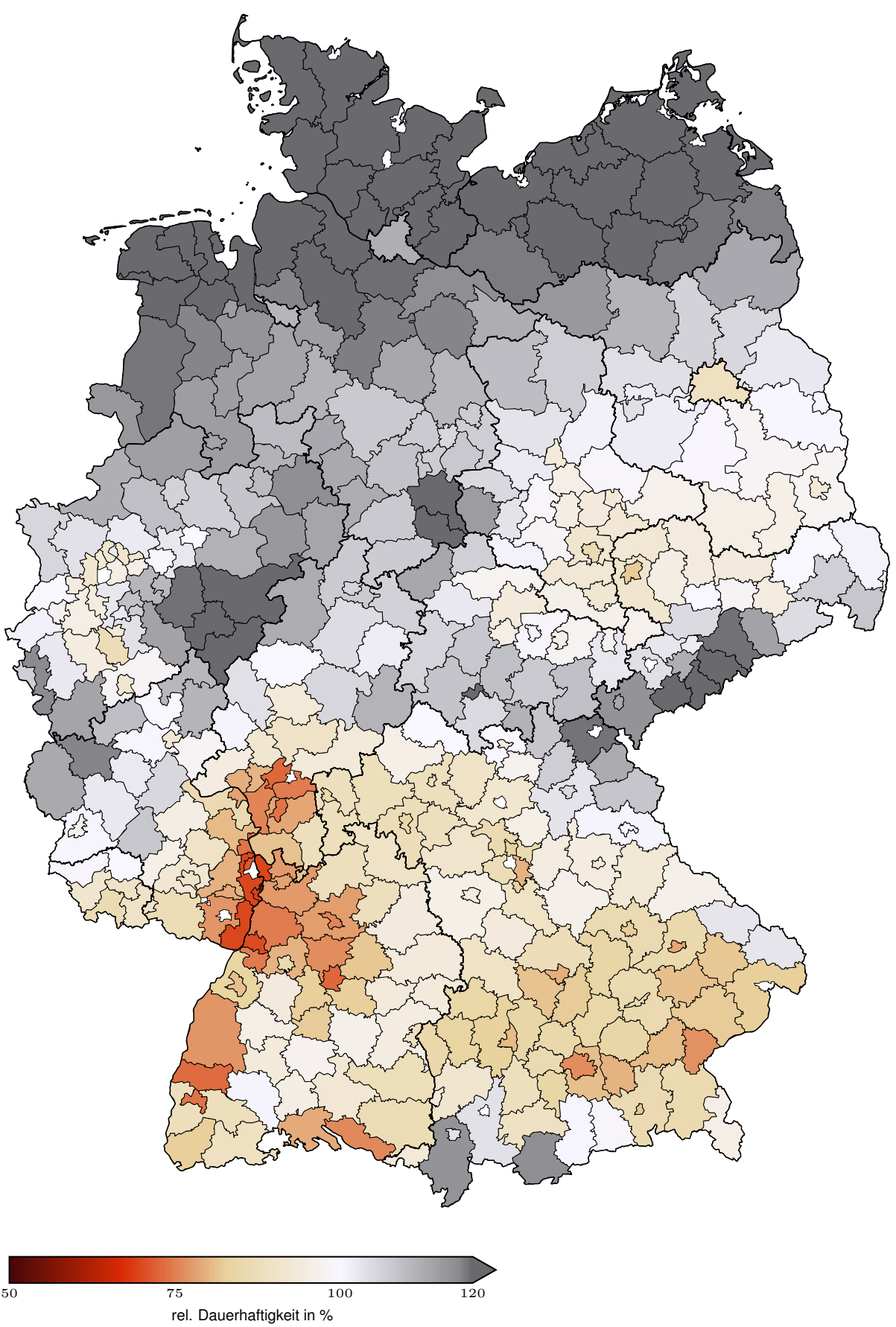

Relative Dauerhaftigkeit für das Gebiet Deutschland auf Basis der Mittelwertfunktionen, normiert auf den Mittelwert aller Landkreise mit der Verkehrsfreigabe 2020, Verkehrsfreigabe 2020 


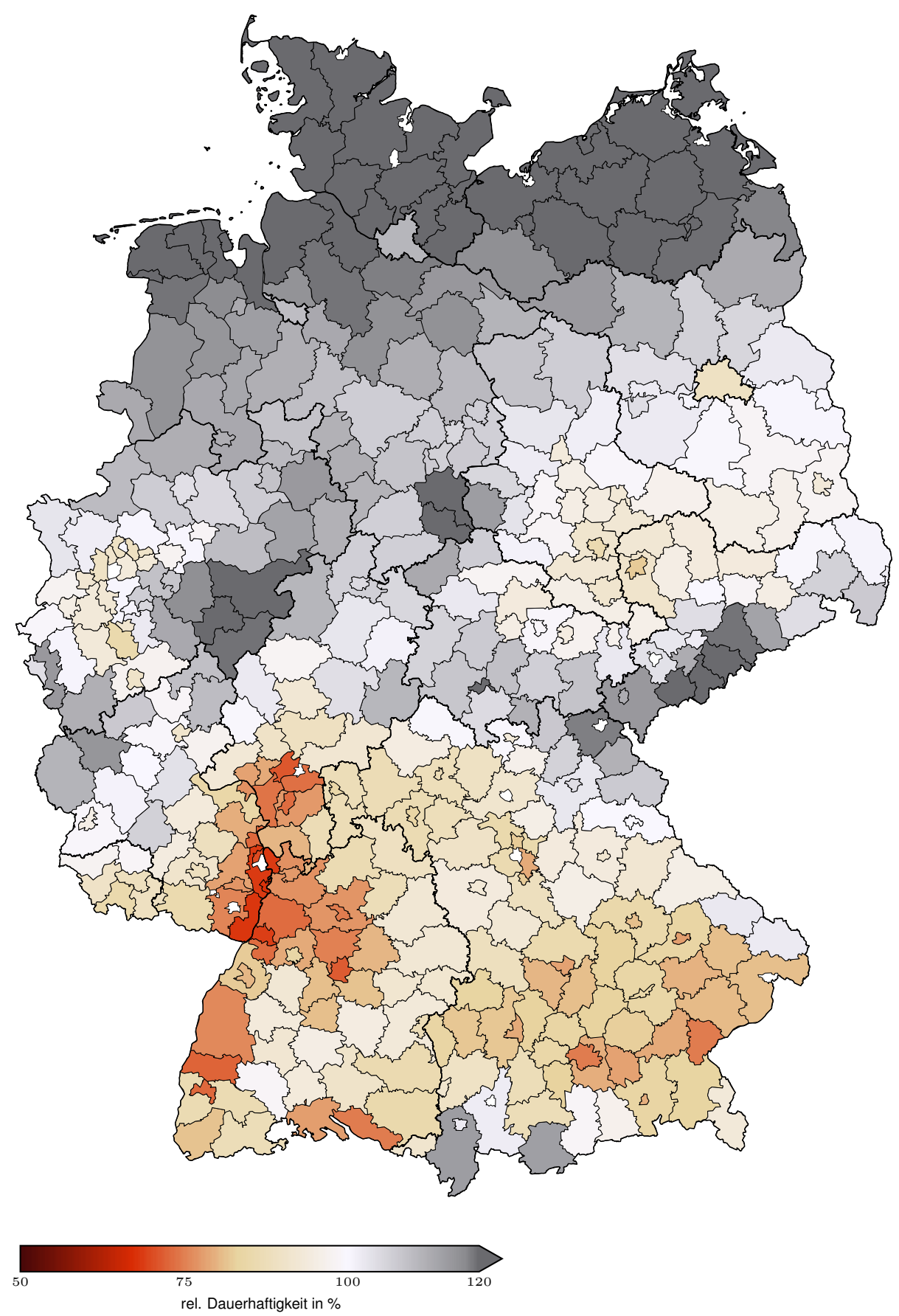

Relative Dauerhaftigkeit für das Gebiet Deutschland auf Basis der Mittelwertfunktionen, normiert auf den Mittelwert aller Landkreise mit der Verkehrsfreigabe 2020, Verkehrsfreigabe 2030 


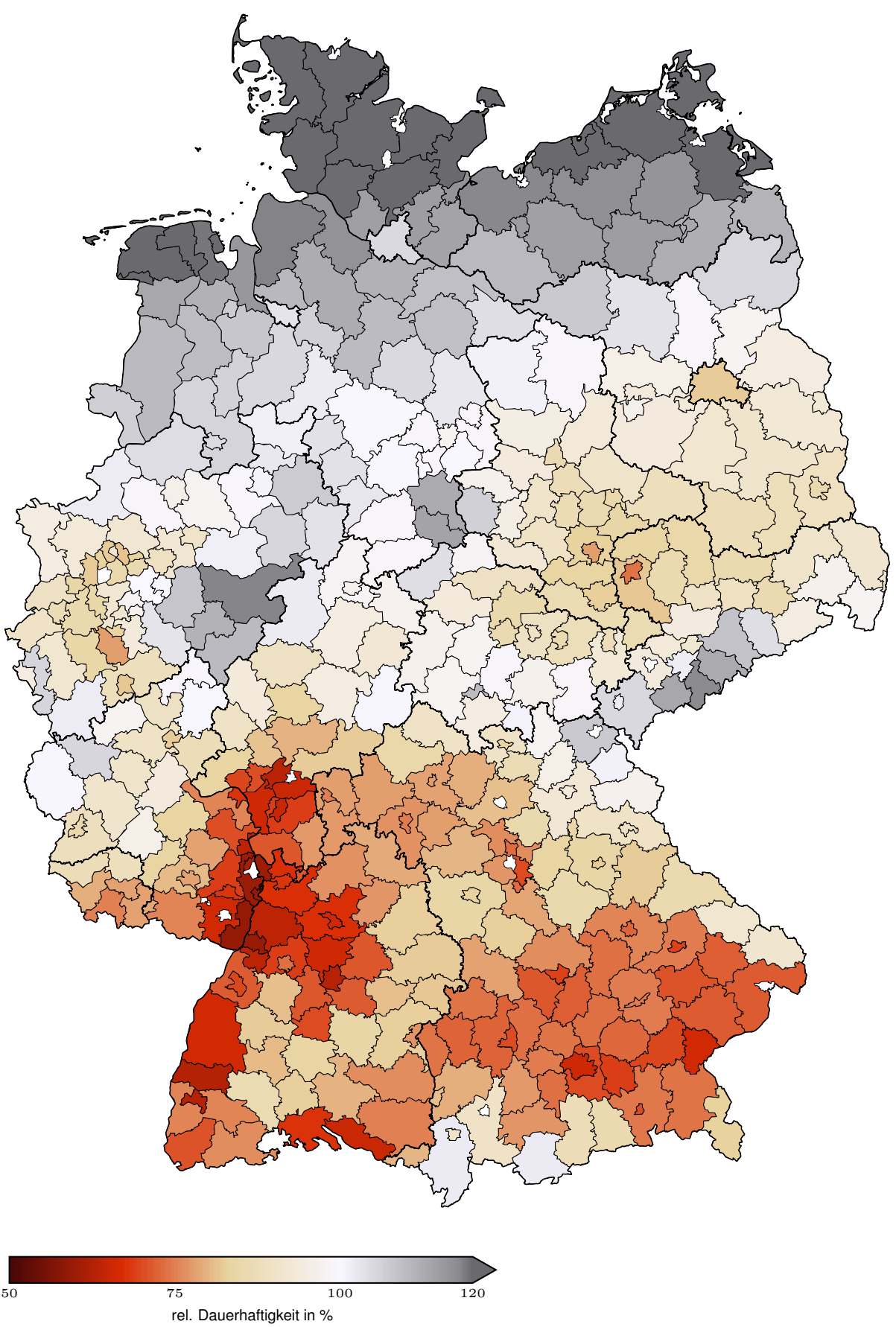

Relative Dauerhaftigkeit für das Gebiet Deutschland auf Basis der Mittelwertfunktionen, normiert auf den Mittelwert aller Landkreise mit der Verkehrsfreigabe 2020, Verkehrsfreigabe 2040 


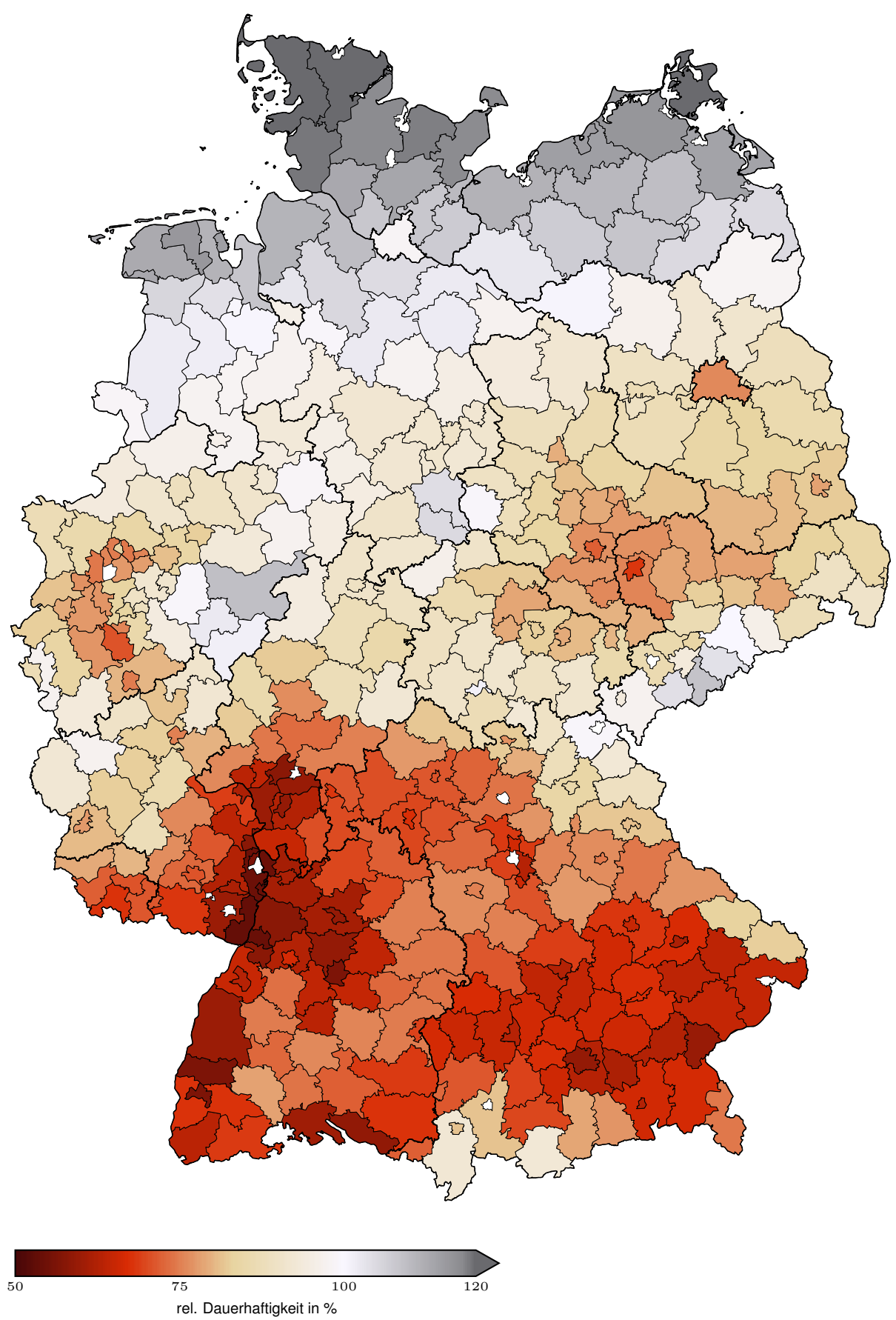

Relative Dauerhaftigkeit für das Gebiet Deutschland auf Basis der Mittelwertfunktionen, normiert auf den Mittelwert aller Landkreise mit der Verkehrsfreigabe 2020, Verkehrsfreigabe 2050 


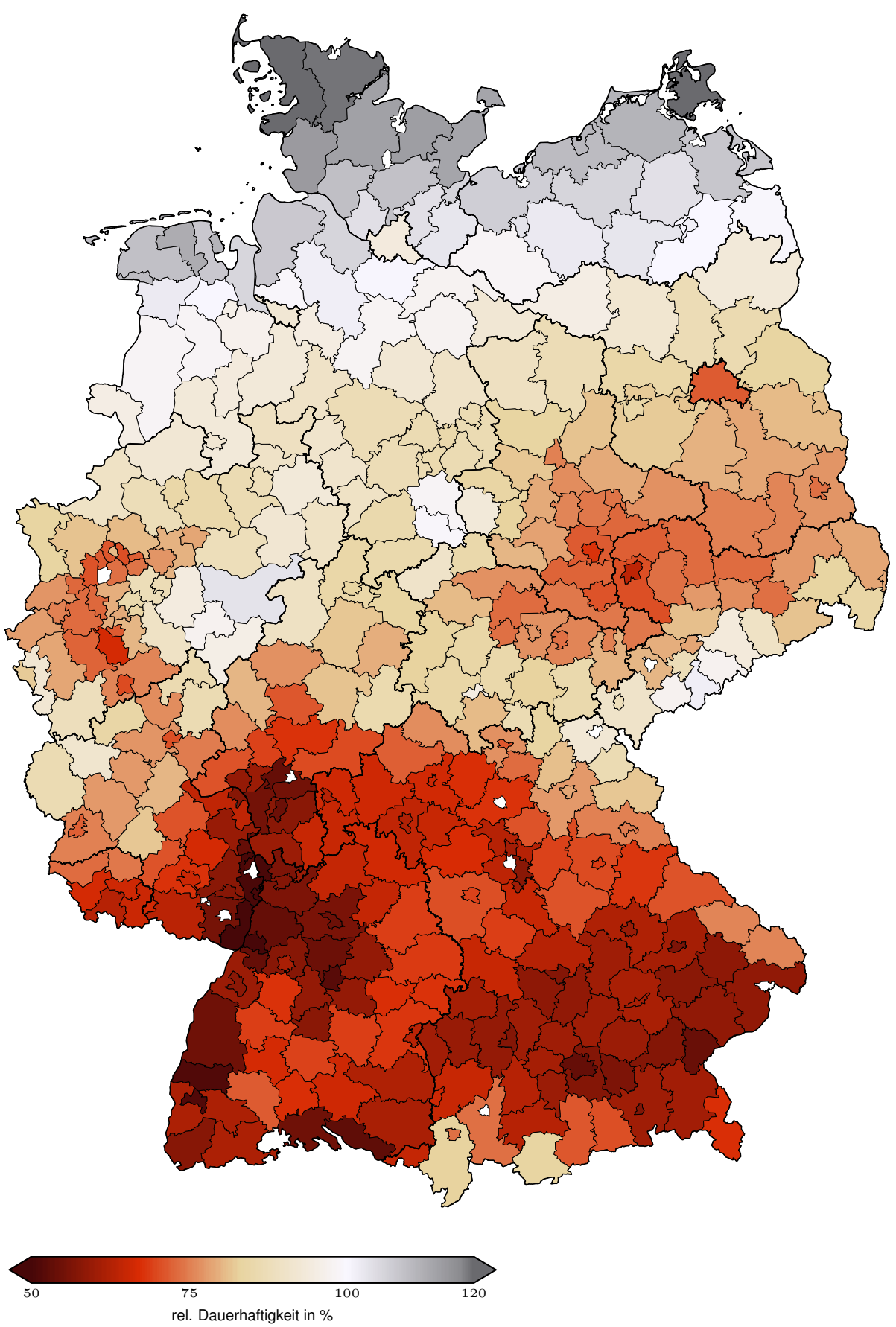

Relative Dauerhaftigkeit für das Gebiet Deutschland auf Basis der Mittelwertfunktionen, normiert auf den Mittelwert aller Landkreise mit der Verkehrsfreigabe 2020, Verkehrsfreigabe 2060 


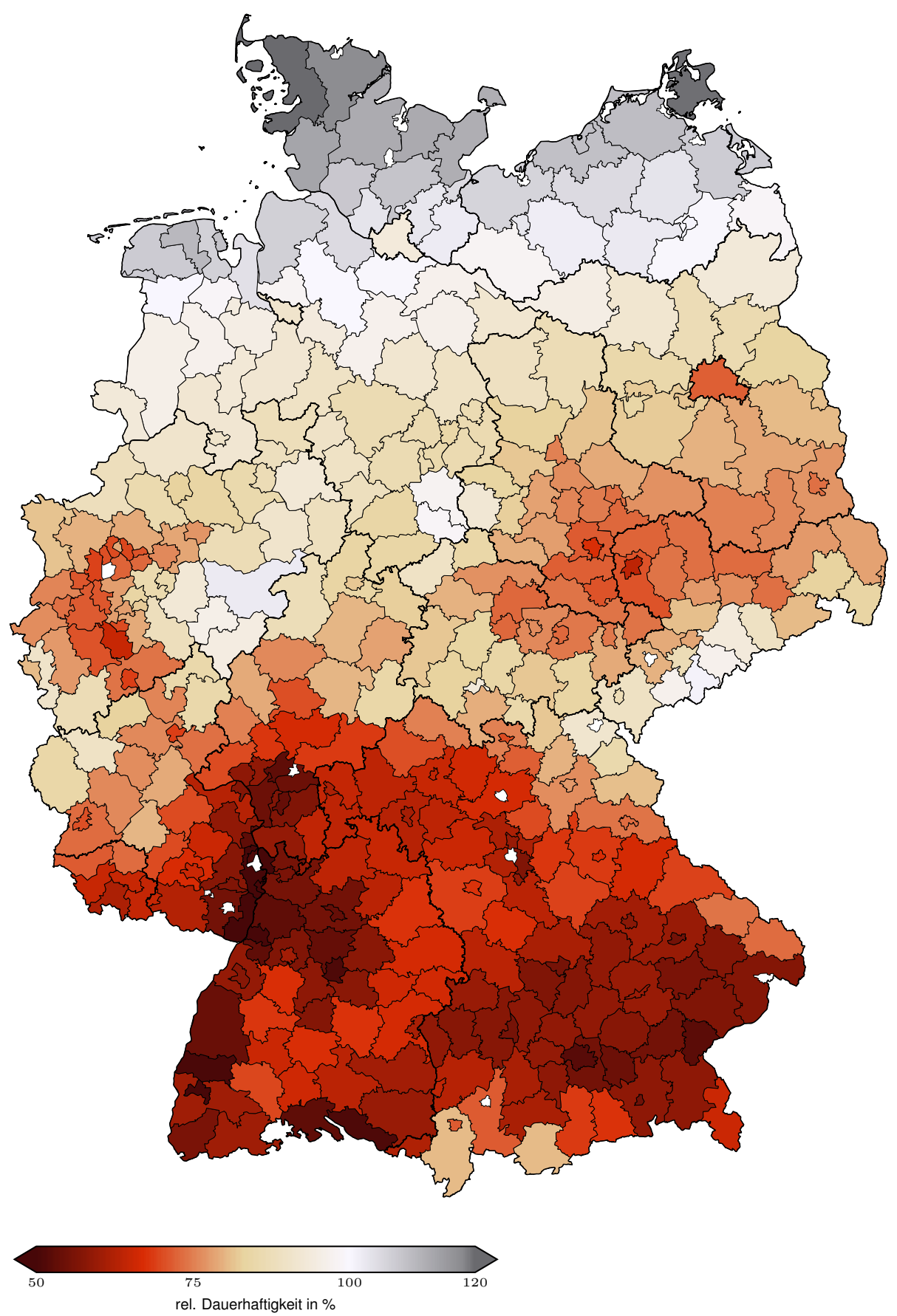

Relative Dauerhaftigkeit für das Gebiet Deutschland auf Basis der Mittelwertfunktionen, normiert auf den Mittelwert aller Landkreise mit der Verkehrsfreigabe 2020, Verkehrsfreigabe 2070 
Anhang

C.2 Spurrinnentiefe 


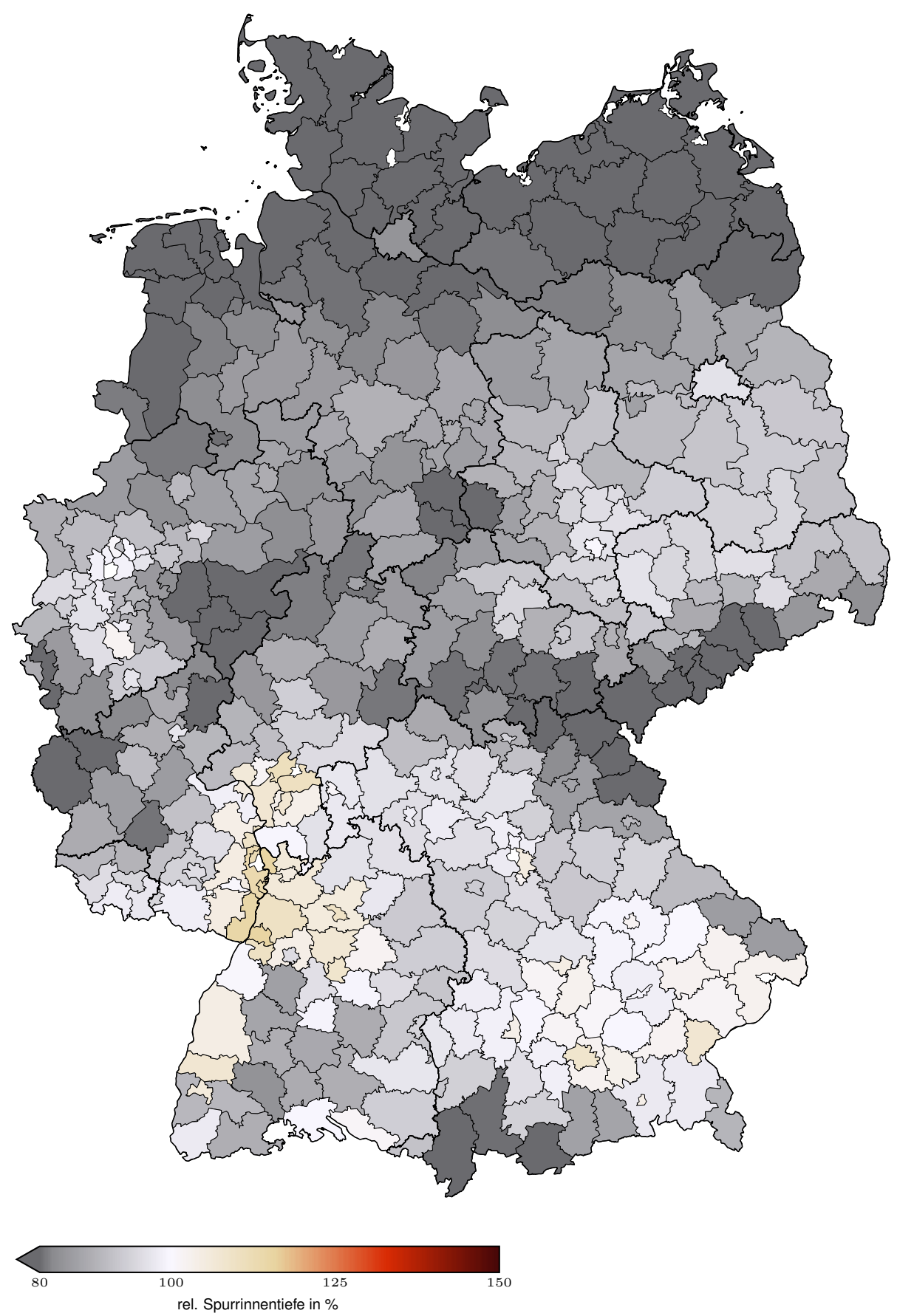

Ermittelte Spurrinnentiefe für das Gebiet Deutschland auf Basis der Mittelwertfunktionen; normiert auf den Mittelwert aller Landkreise mit Verkehrsfreigabe ab 2020, Verkehrsfreigabe 1960 


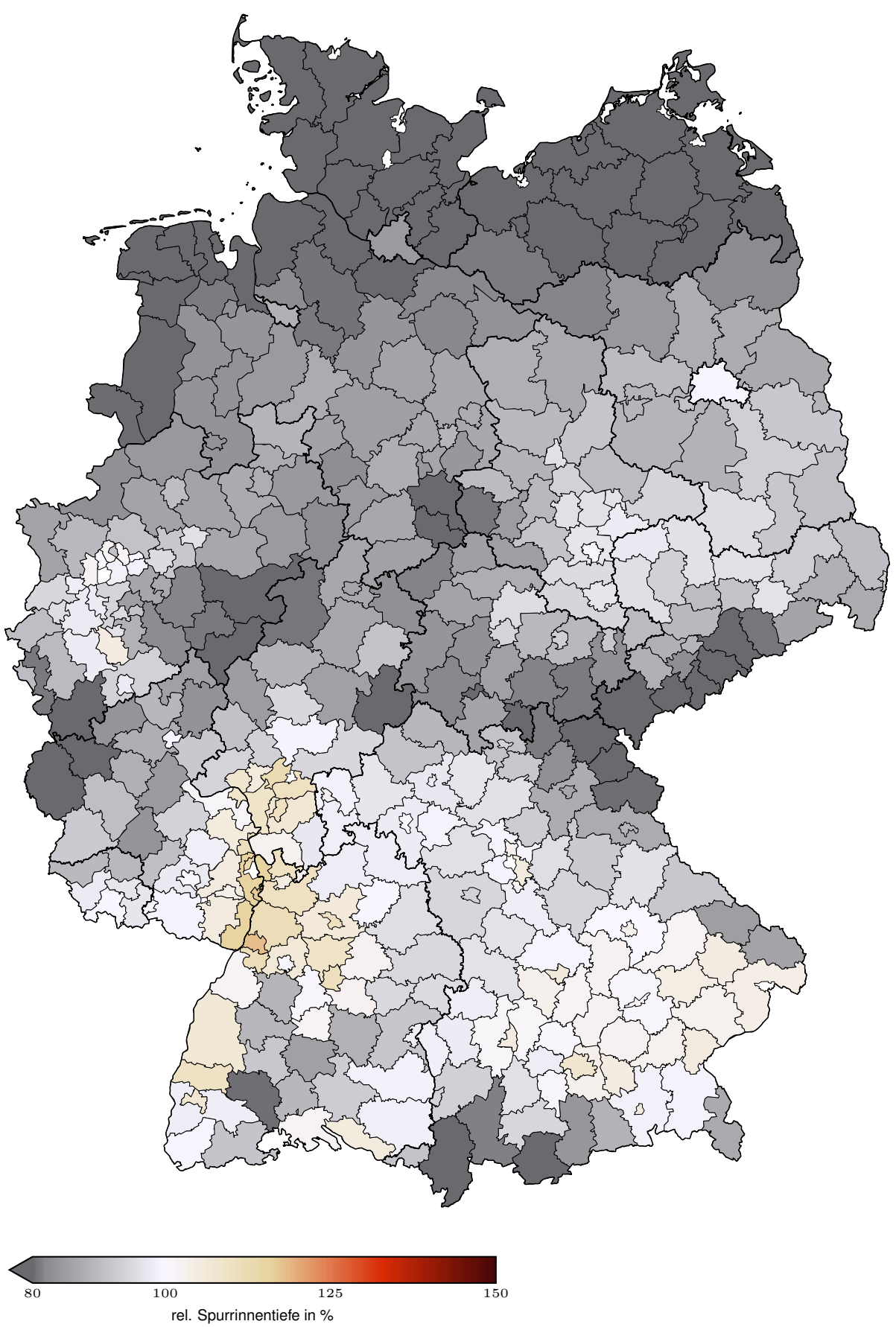

Ermittelte Spurrinnentiefe für das Gebiet Deutschland auf Basis der Mittelwertfunktionen; normiert auf den Mittelwert aller Landkreise mit Verkehrsfreigabe ab 2020, Verkehrsfreigabe 1970 


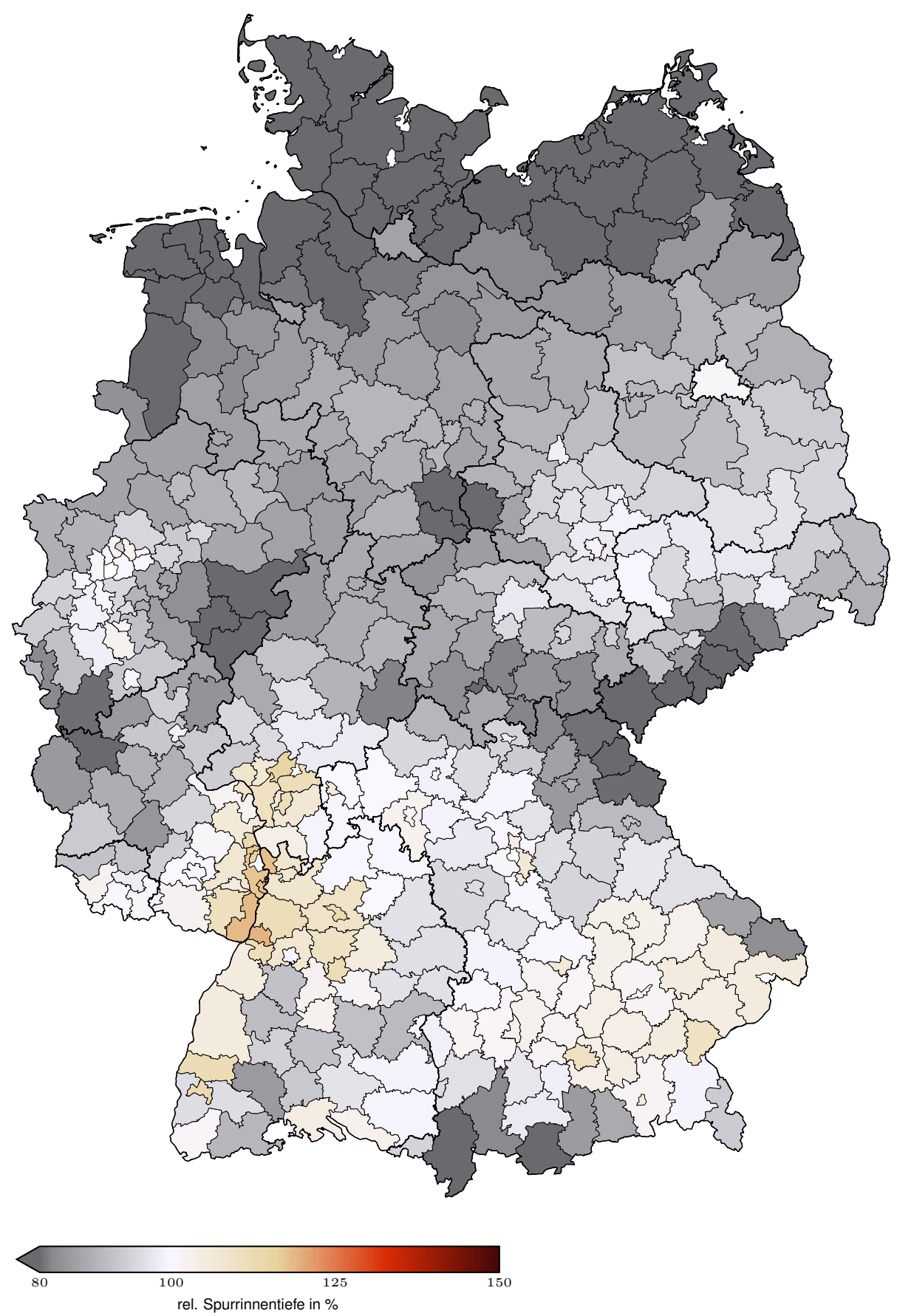

Ermittelte Spurrinnentiefe für das Gebiet Deutschland auf Basis der Mittelwertfunktionen; normiert auf den Mittelwert aller Landkreise mit Verkehrsfreigabe ab 2020, Verkehrsfreigabe 1980 


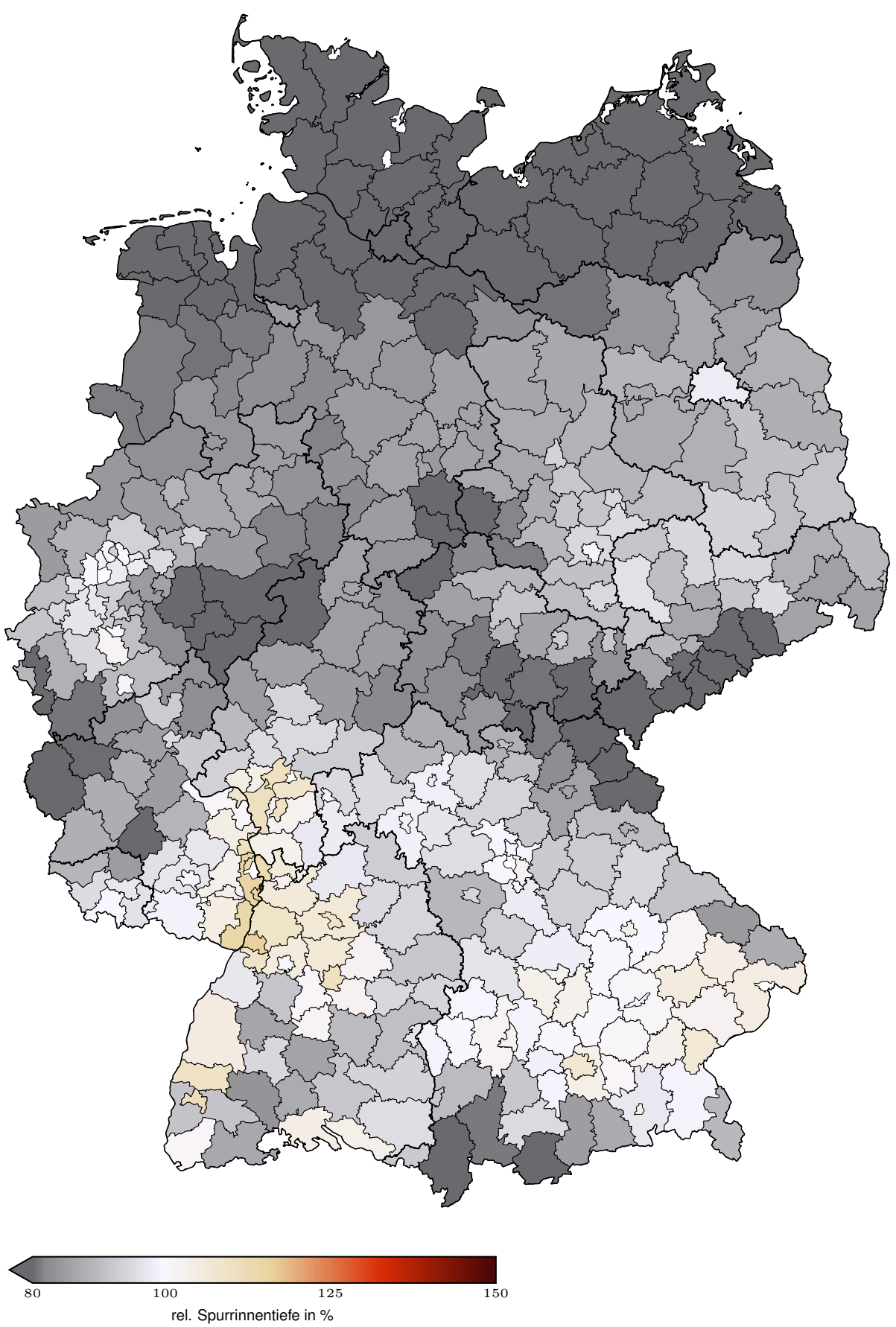

Ermittelte Spurrinnentiefe für das Gebiet Deutschland auf Basis der Mittelwertfunktionen; normiert auf den Mittelwert aller Landkreise mit Verkehrsfreigabe ab 2020, Verkehrsfreigabe 1990 


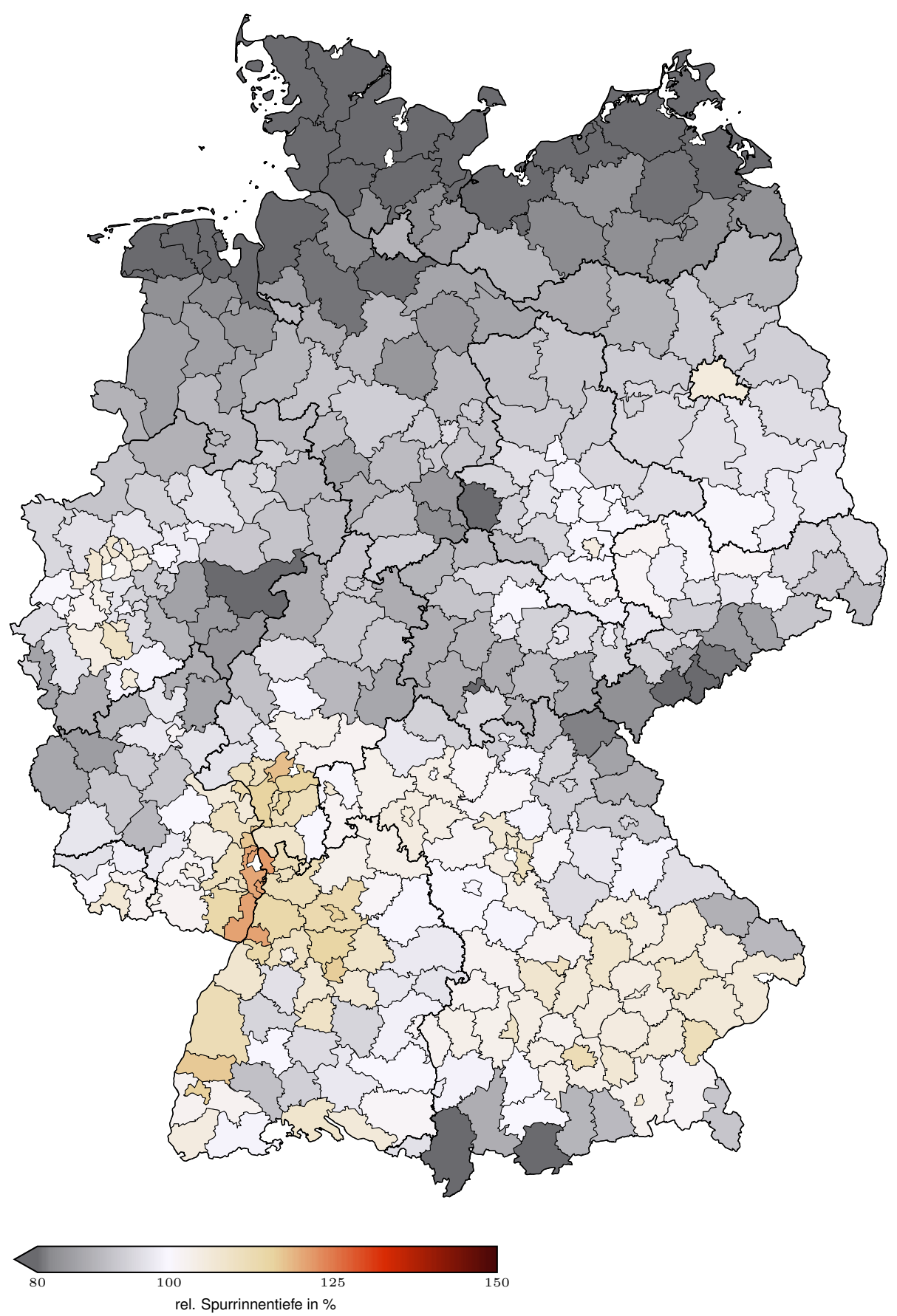

Ermittelte Spurrinnentiefe für das Gebiet Deutschland auf Basis der Mittelwertfunktionen; normiert auf den Mittelwert aller Landkreise mit Verkehrsfreigabe ab 2020, Verkehrsfreigabe 2000 


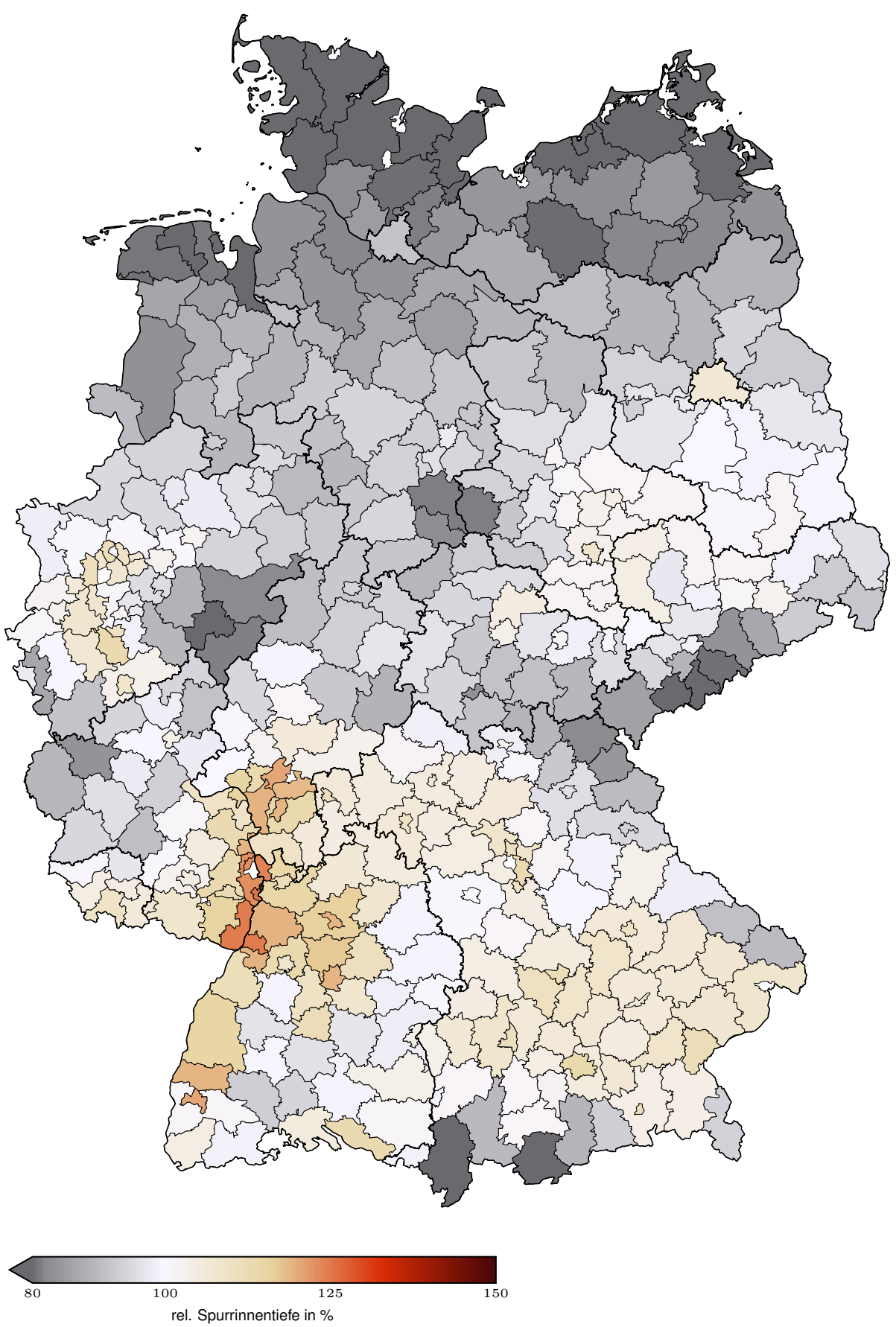

Ermittelte Spurrinnentiefe für das Gebiet Deutschland auf Basis der Mittelwertfunktionen; normiert auf den Mittelwert aller Landkreise mit Verkehrsfreigabe ab 2020, Verkehrsfreigabe 2010 


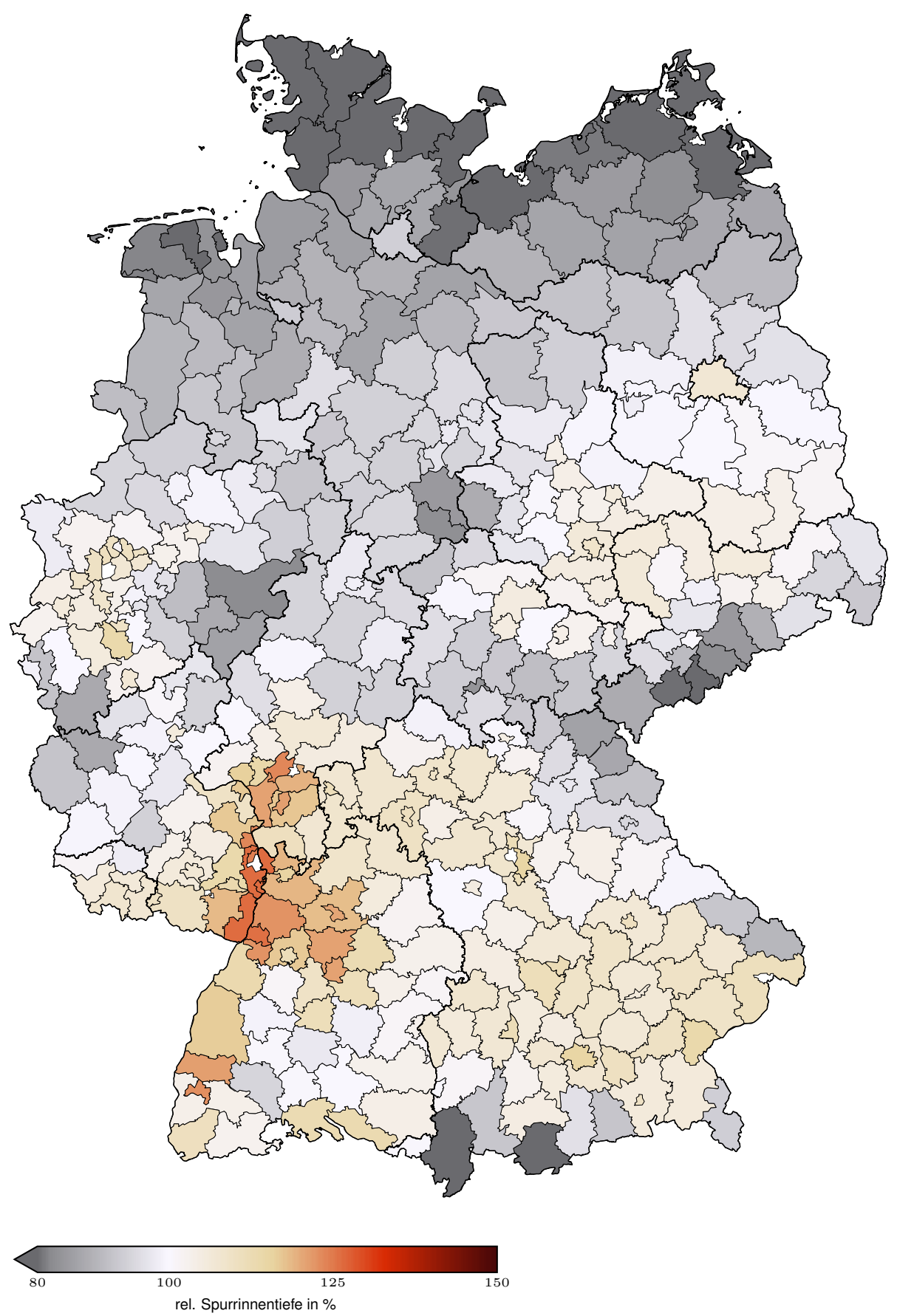

Ermittelte Spurrinnentiefe für das Gebiet Deutschland auf Basis der Mittelwertfunktionen; normiert auf den Mittelwert aller Landkreise mit Verkehrsfreigabe ab 2020, Verkehrsfreigabe 2020 


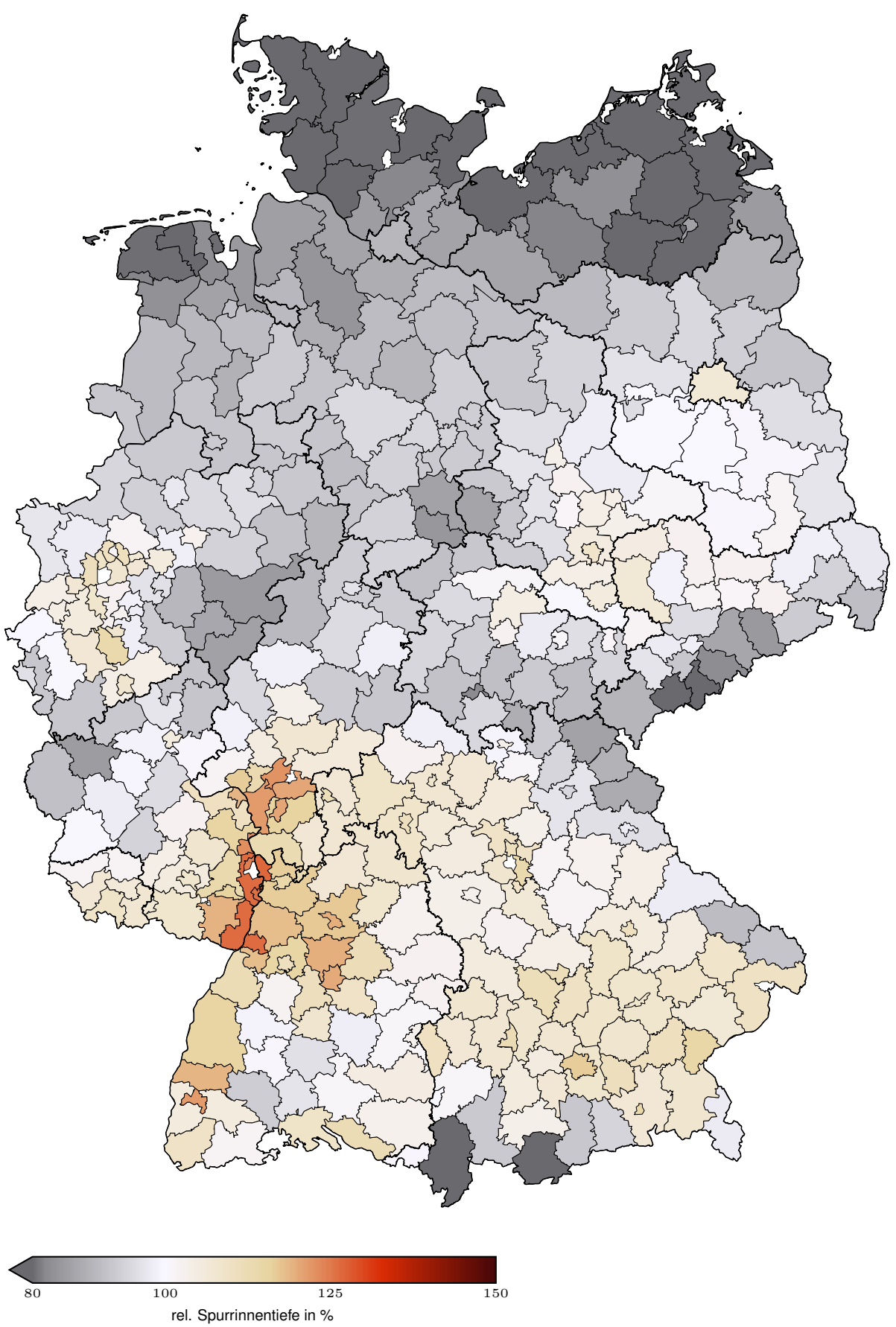

Ermittelte Spurrinnentiefe für das Gebiet Deutschland auf Basis der Mittelwertfunktionen; normiert auf den Mittelwert aller Landkreise mit Verkehrsfreigabe ab 2020, Verkehrsfreigabe 2030 


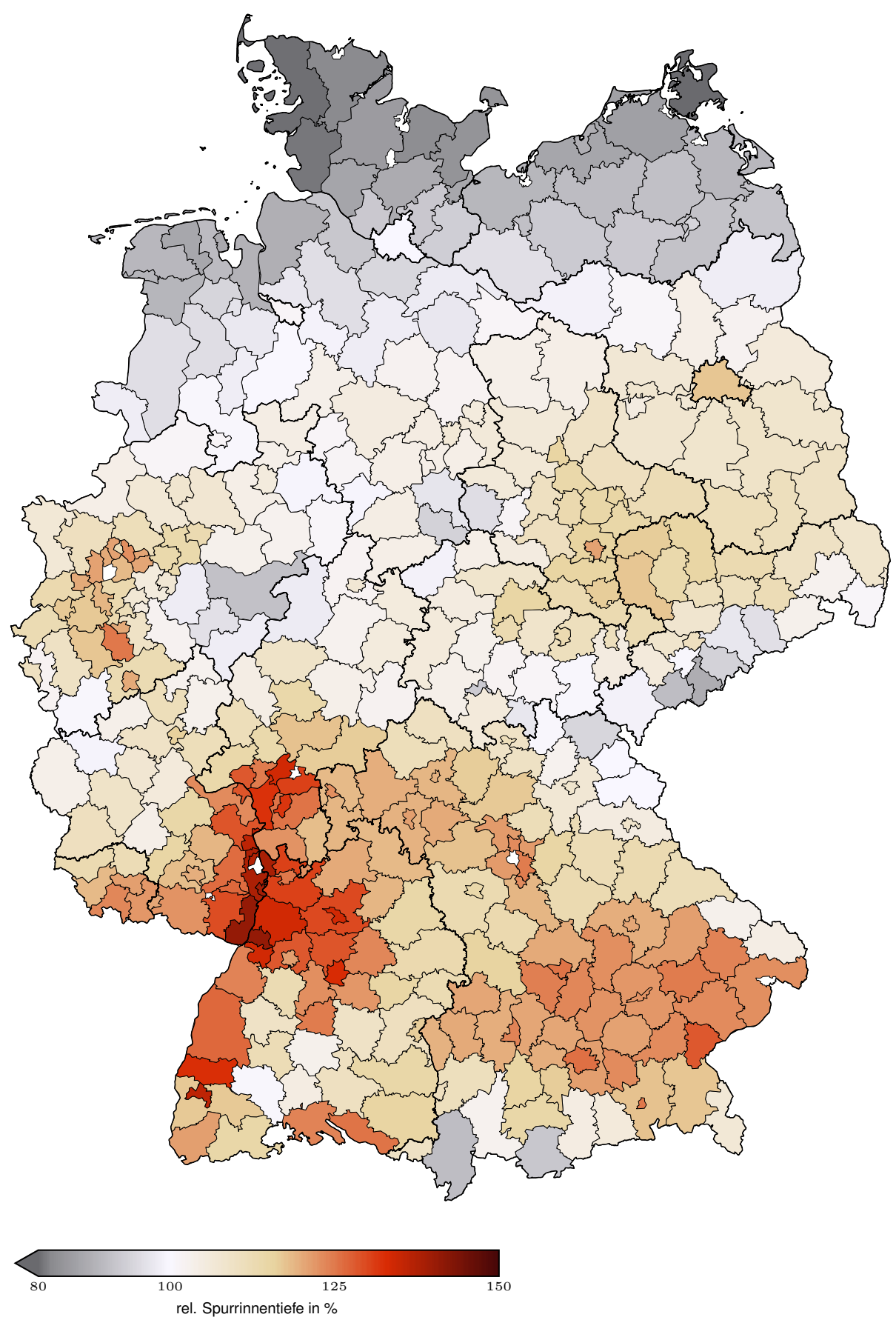

Ermittelte Spurrinnentiefe für das Gebiet Deutschland auf Basis der Mittelwertfunktionen; normiert auf den Mittelwert aller Landkreise mit Verkehrsfreigabe ab 2020, Verkehrsfreigabe 2040 


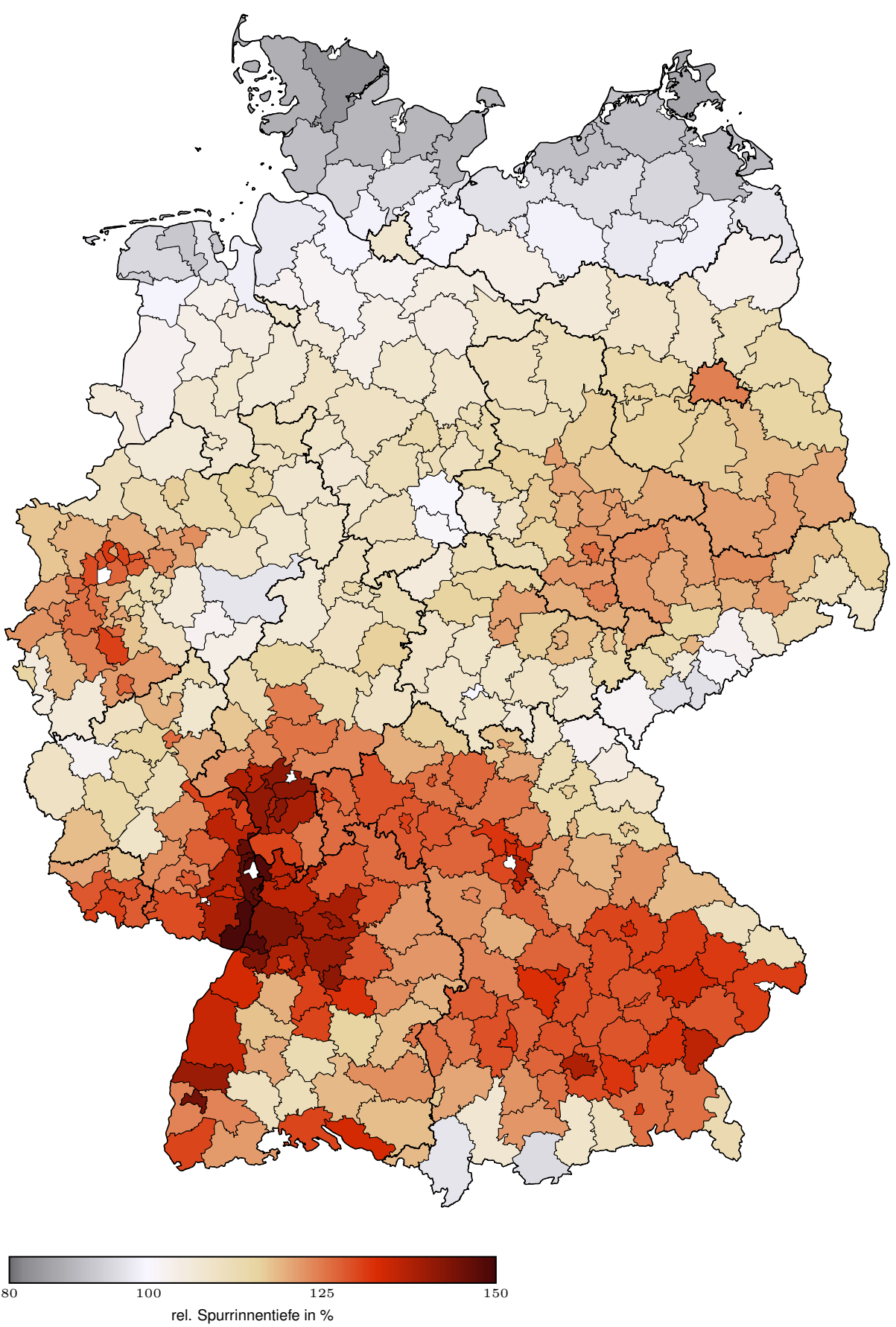

Ermittelte Spurrinnentiefe für das Gebiet Deutschland auf Basis der Mittelwertfunktionen; normiert auf den Mittelwert aller Landkreise mit Verkehrsfreigabe ab 2020, Verkehrsfreigabe 2050 


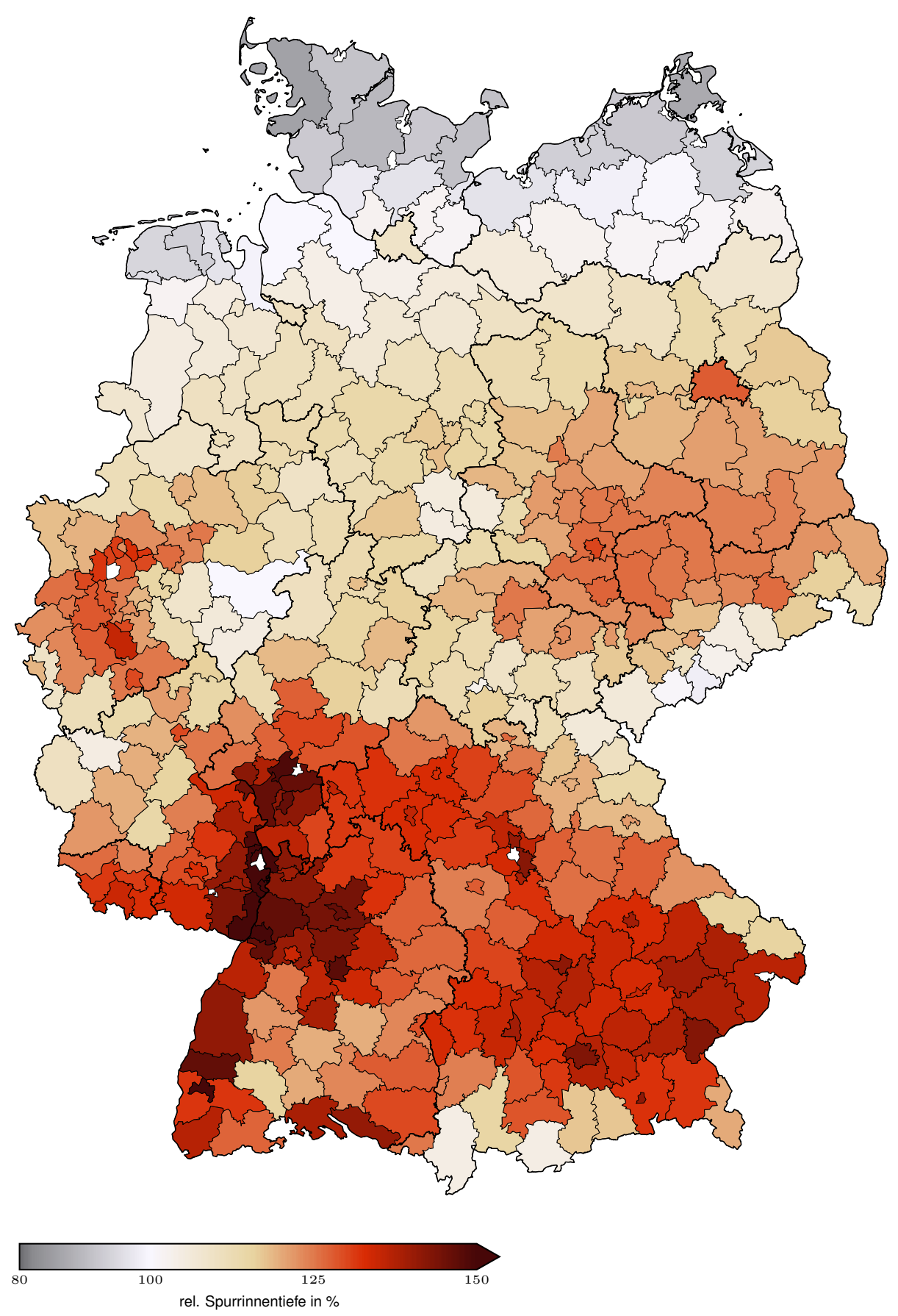

Ermittelte Spurrinnentiefe für das Gebiet Deutschland auf Basis der Mittelwertfunktionen; normiert auf den Mittelwert aller Landkreise mit Verkehrsfreigabe ab 2020, Verkehrsfreigabe 2060 


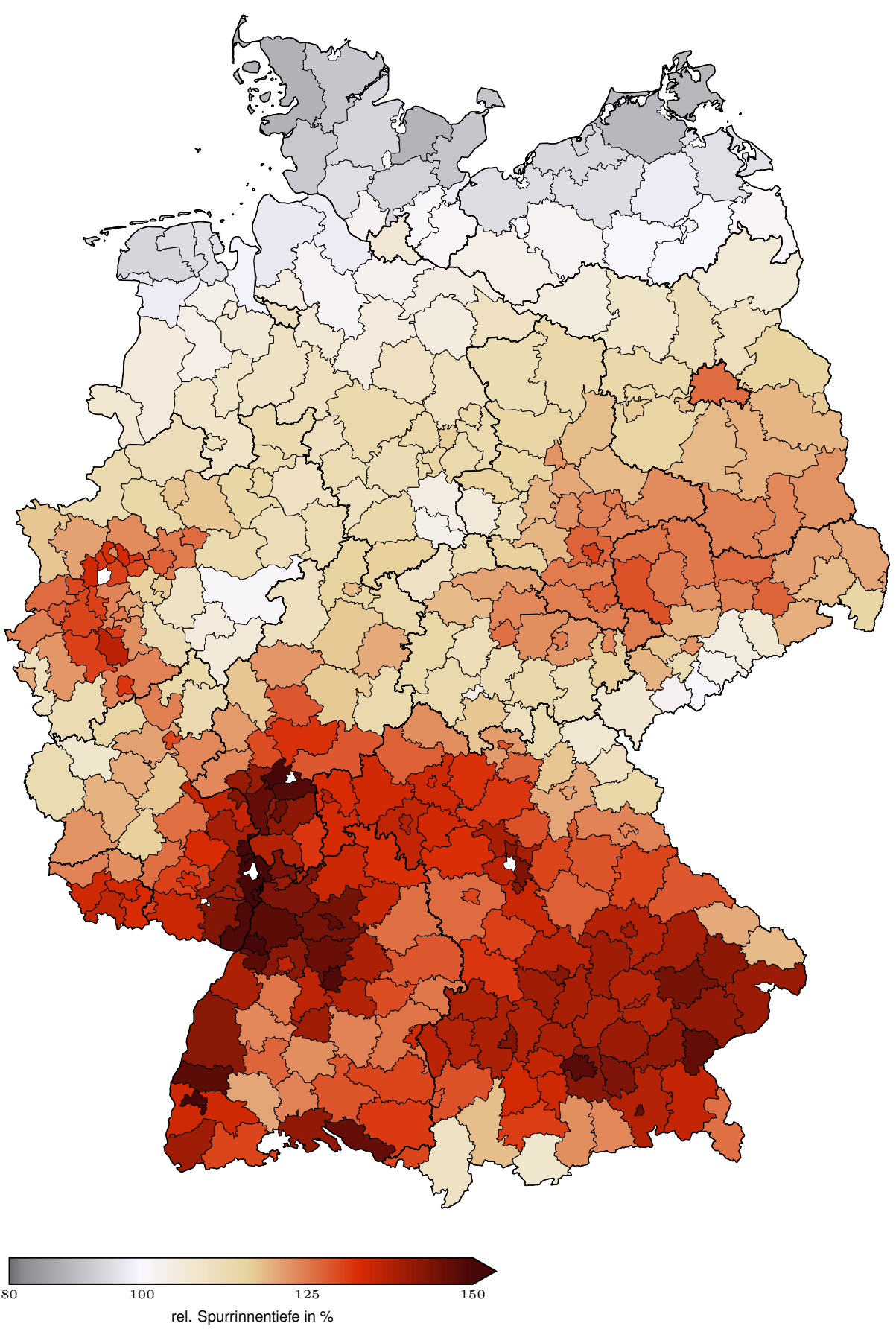

Ermittelte Spurrinnentiefe für das Gebiet Deutschland auf Basis der Mittelwertfunktionen; normiert auf den Mittelwert aller Landkreise mit Verkehrsfreigabe ab 2020, Verkehrsfreigabe 2070 
D Anpassungsstrategie 


\section{D.1 Fallbeispiel PCM}

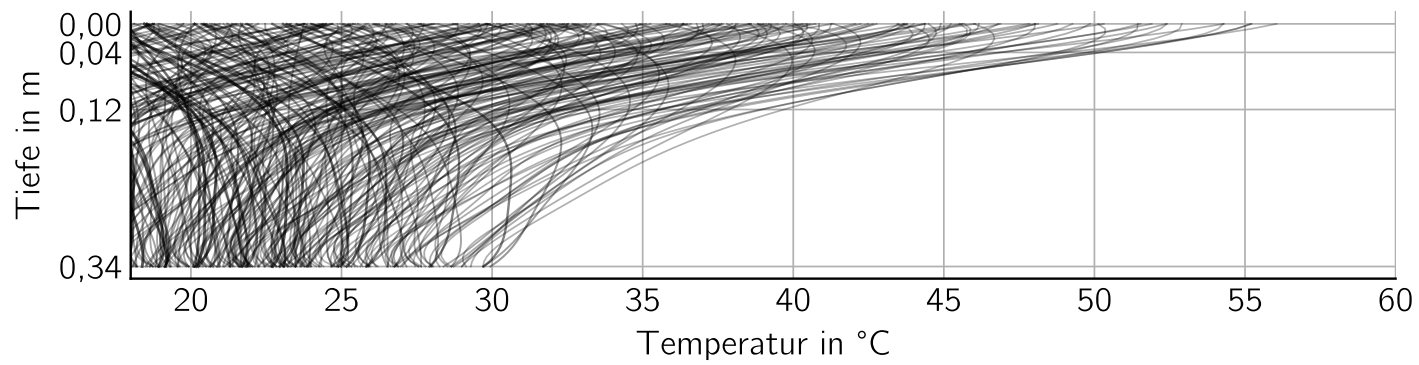

Verlauf der Temperaturbedingungen auf Basis der Mittelwertfunktionen, Referenzvariante

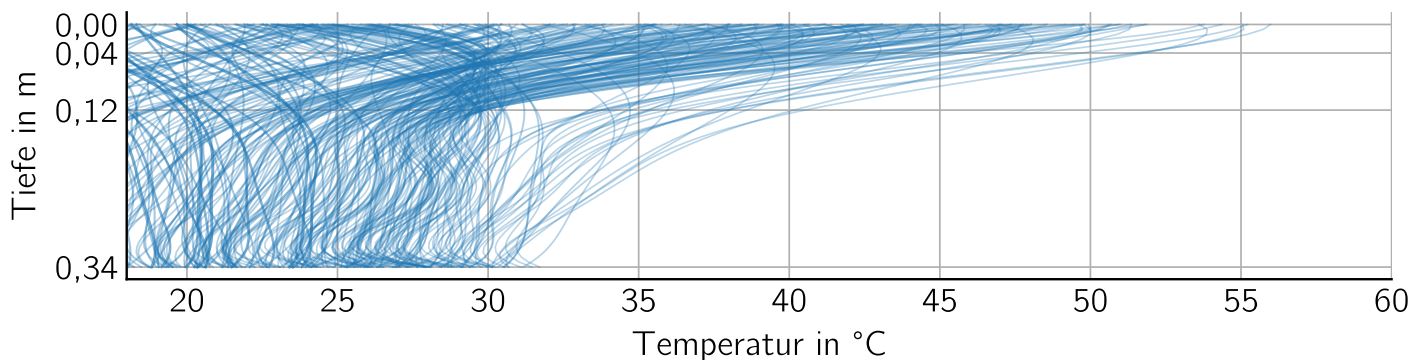

Verlauf der Temperaturbedingungen auf Basis der Mittelwertfunktionen, Schmelztemperatur bei $30^{\circ} \mathrm{C}$ 


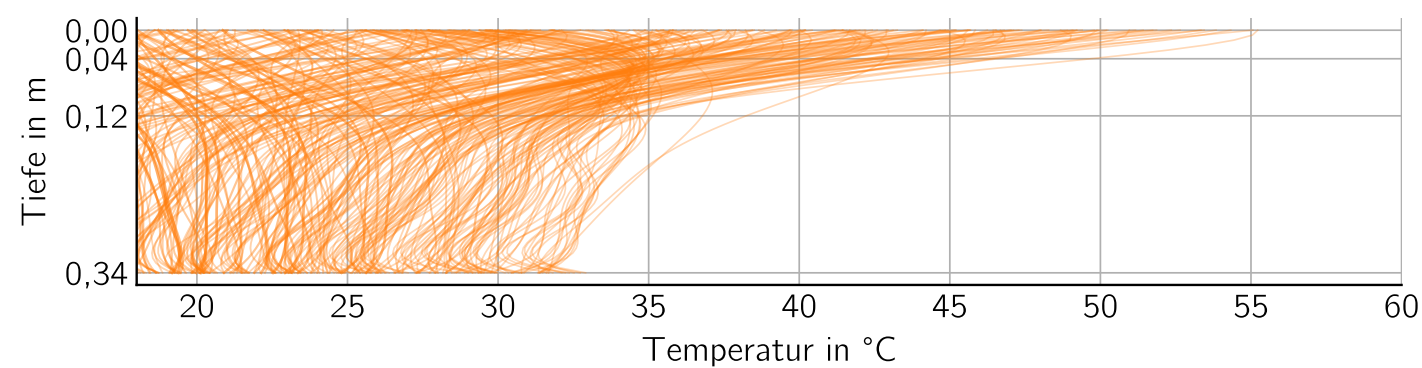

Verlauf der Temperaturbedingungen auf Basis der Mittelwertfunktionen, Schmelztemperatur bei $35^{\circ} \mathrm{C}$

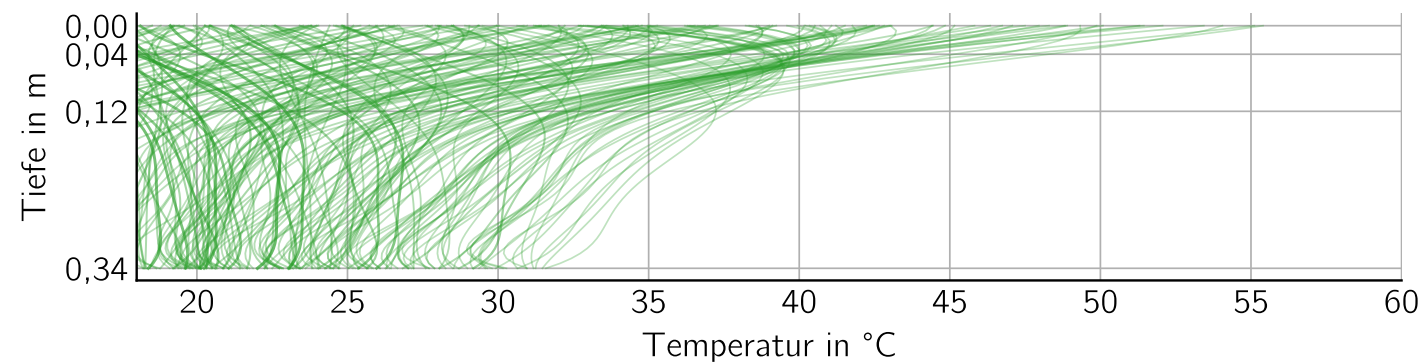

Verlauf der Temperaturbedingungen auf Basis der Mittelwertfunktionen, Schmelztemperatur bei $40^{\circ} \mathrm{C}$

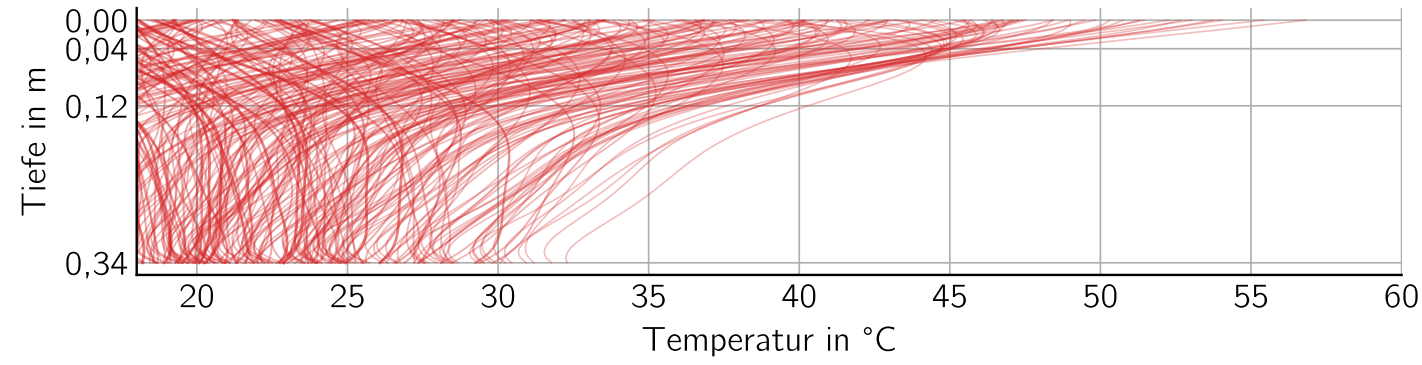

Verlauf der Temperaturbedingungen auf Basis der Mittelwertfunktionen, Schmelztemperatur bei $45^{\circ} \mathrm{C}$ 


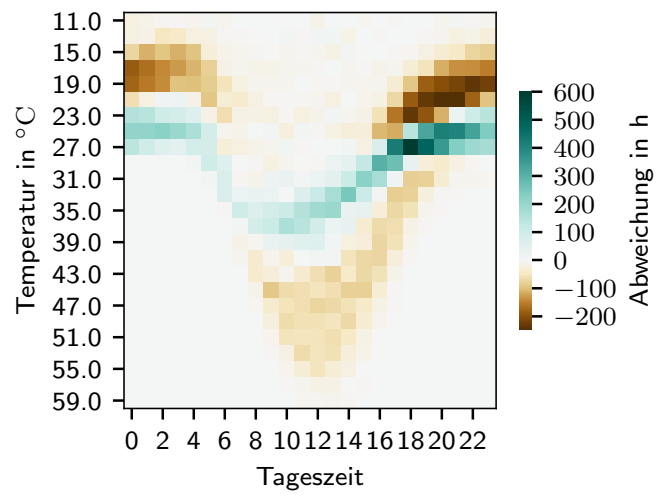

(a) Schmelzpunkt $30,00^{\circ} \mathrm{C}$

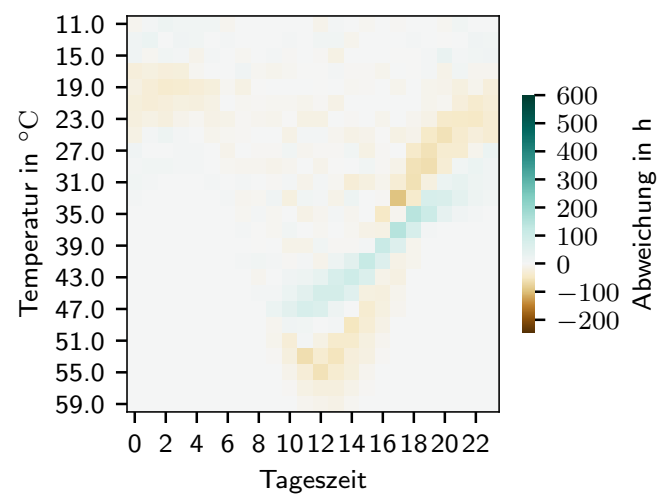

(c) Schmelzpunkt $40,00^{\circ} \mathrm{C}$

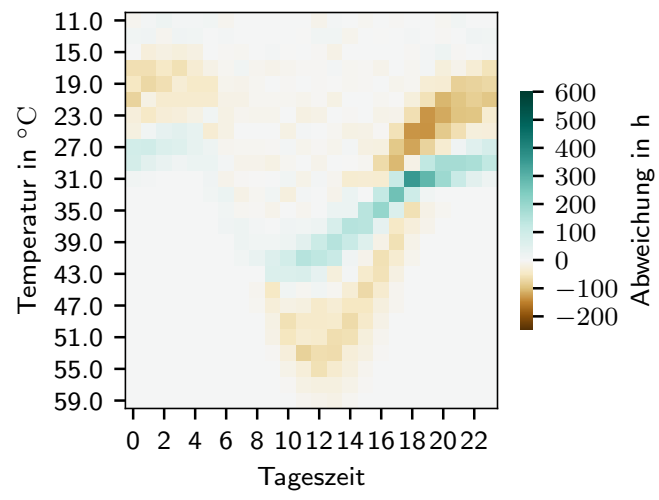

(b) Schmelzpunkt $35,00^{\circ} \mathrm{C}$

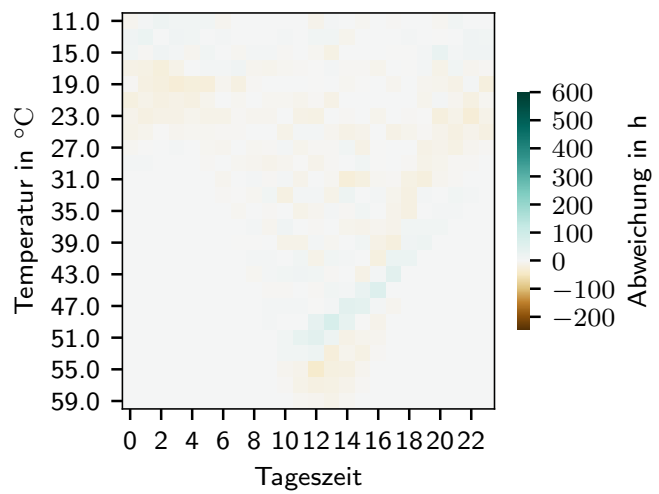

(d) Schmelzpunkt $45,00^{\circ} \mathrm{C}$

Abweichung der Temperaturen der PCM-Varianten bezogen auf die Referenzvariante, Asphaltdeckschicht 


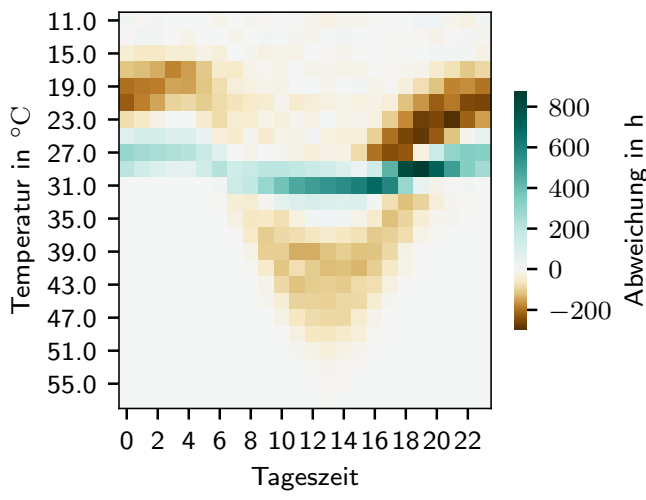

(e) Schmelzpunkt $30,00^{\circ} \mathrm{C}$

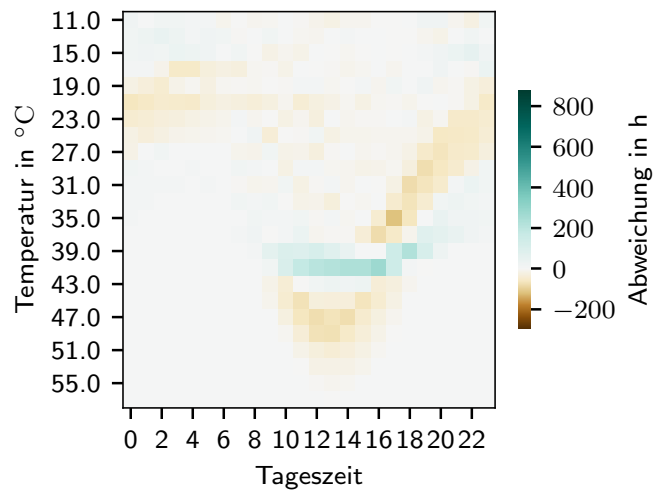

(g) Schmelzpunkt $40,00^{\circ} \mathrm{C}$

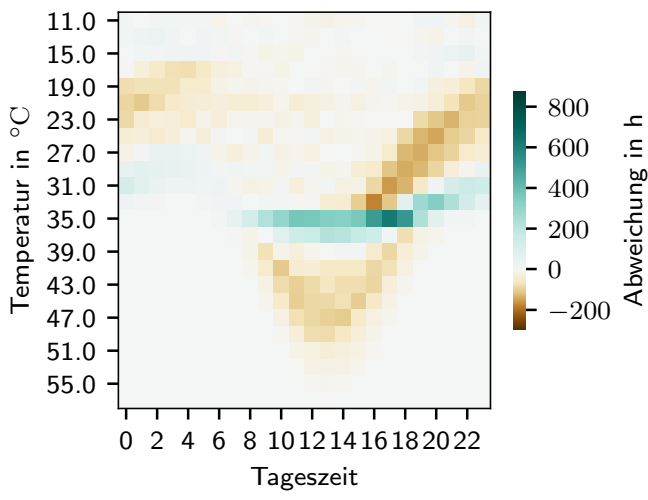

(f) Schmelzpunkt $35,00^{\circ} \mathrm{C}$

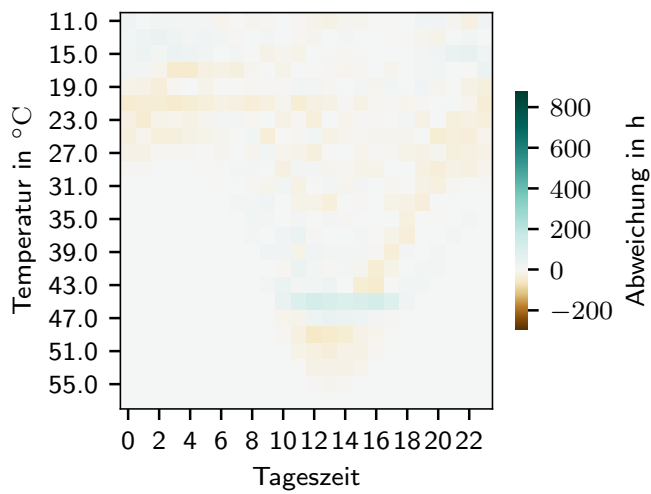

(h) Schmelzpunkt $45,00^{\circ} \mathrm{C}$

Abweichung der Temperaturen der PCM-Varianten bezogen auf die Referenzvariante, Asphaltbinderschicht 


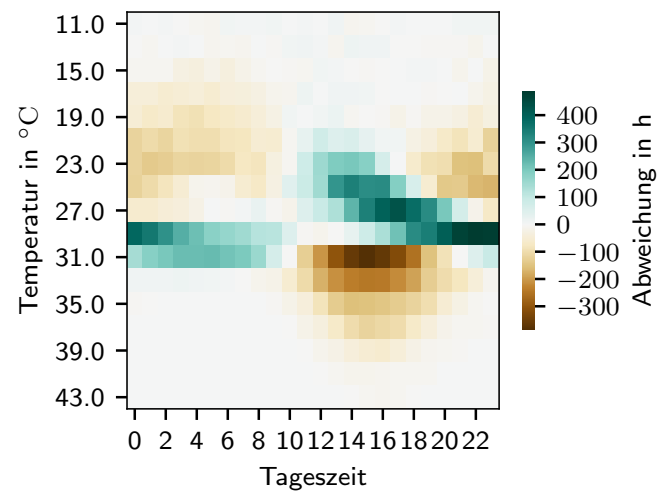

(i) Schmelzpunkt $30,00^{\circ} \mathrm{C}$

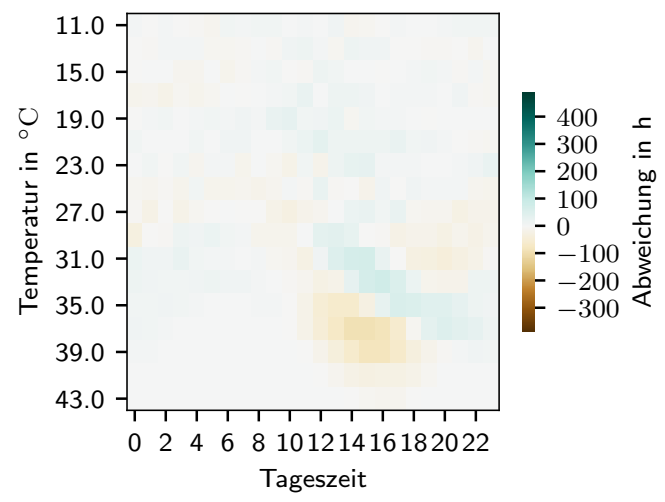

(k) Schmelzpunkt $40,00^{\circ} \mathrm{C}$

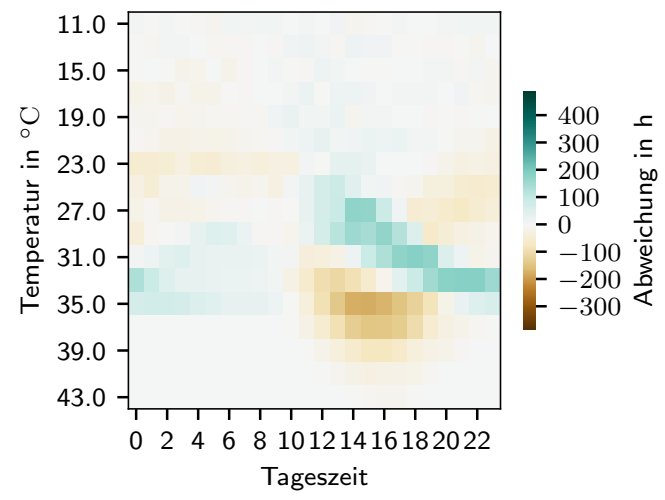

(j) Schmelzpunkt $35,00^{\circ} \mathrm{C}$

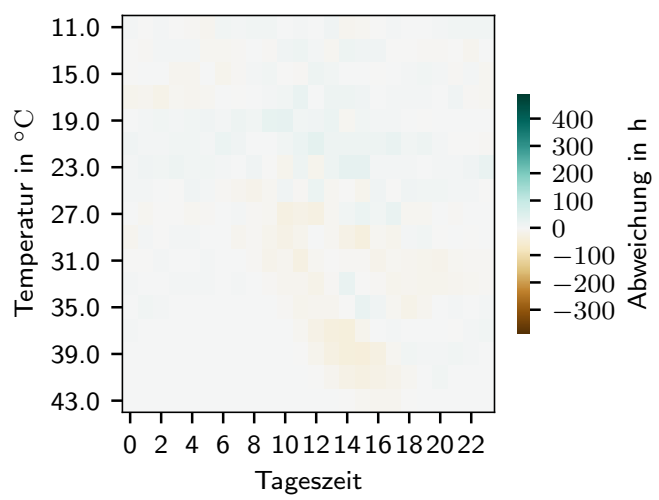

(I) Schmelzpunkt $45,00^{\circ} \mathrm{C}$

Abweichung der Temperaturen der PCM-Varianten bezogen auf die Referenzvariante, Asphalttragschicht 